\section{Plenary and Keynote Speakers Summaries}

\section{INS-V01 \\ Cornea Banking und Möglichkeiten des Tissue Engineerings}

\section{${ }^{*} K$. Engelmann}

Klinikum Chemnitz gGmbH, Klinik für Augenheilkunde, Chemnitz, Germany

INS-V02

Patient Blood Management aus anästhesiologischer Sicht

${ }^{*}$ H.K. van Aken

Universitätsklinikum Münster, Klinik für Anästhesiologie, operative Intensivmedizin und Schmerztherapie, Münster, Germany

INS-V03

Moderne Polytraumaversorgung im Rahmen des Traumanetzwerks

\section{*M.J. Raschke}

Klinik und Poliklinik für Unfall-, Hand- und Wiederherstellungschirurgie, Münster, Germany

INS-V04

\section{Konzepte für eine rational-basierte Hämotherapie}

\section{${ }^{*}$ P. Schlenke}

Universitätsklinikum Münster, Institut für Transfusionsmedizin und Transplantationsimmunologie, Münster, Germany

INS-V05

\section{Prävention und Therapie der traumaassoziierten Koagulopathie}

\section{${ }^{*}$ C. von Heymann}

Charité-Universitätsmedizin Berlin, Klinik für Anästhesiologie mit Schwerpunkt operative Intensivmedizin, Berlin, Germany

INS-V06

\section{Indikationen zur Thrombozytentransfusion}

${ }^{*} A$. Greinacher

Universitätsmedizin Greifswald, Abteilung für Transfusionsmedizin, Greifswald, Germany
INS-V07

\section{Granulozytentransfusion}

\section{${ }^{*} U$. Sachs}

Univ.-Klinikum Gießen-Marburg , Institut für Klinische Immunologie und Transfusionsmedizin , Gießen, Germany

INS-V08

Treatment of Immune Hemolysis

\section{${ }^{*}$ A. Salama}

Campus Virchow Klinikum, ZTB Zentrum für Transfusionsmedizin und Zelltherapie Berlin gemeinnützige $\mathrm{GmbH}$, Berlin, Germany

Immune hemolytic anemia is the best characterized immune disease. Nevertheless, the disease is heterogeneous, and we may infrequently loose patients due to inadequate treatment and/or in adequate response to treatment. Immune hemolysis is classified in alloimmune haemolytic anemia, autoimmune hemolytic anemia (AIHA) of warm type, AIHA of cold types, and drug induced forms. The majority of cases are related to AIHA of warm type. A specific therapy for this form is yet not available, and current treatment options are largely experience-based and not evidence-based. There are no randomized studies, and there are no formal guide lines for treatment of this disease.

Initially, steroids are usually given to all patients. However some patients can not be treated with steroids, and the vast majority of patients require additional drugs. Currently, the following drugs are used in treatment of AIHA: azathioprine, cyclophosphamide and rituximab. In addition, there is evidance that erythropoietin may also help in management of AIHA. Unlike splenectomy in autoimmune thrombocytopenia, splenectomy in AIHA is less effective, and is rarely indicated. The question which drug or which drug combination should be used in treatment of AIHA depends on the affected patent (age, sex, and severity of hemolysis).

Most importantly, patients with hypoxia due to anemia may acutely require blood transfusion. In such cases, blood transfusion is the best and most rapid effective treatment, even in cases with positive cross-maches due to autoantibodies.

The most effective therapy in patients with AIHA of cold types is the consistent avoidance of exposure to cold. Some patients with severe AIHA of cold type may response to rituximab, but there is limited information on long-term efficacy and side effects.

INS-V09

\section{RBC storage studies come of age}

${ }^{*}$ L. van de Watering

Sanquin - Leiden University Medical Centre, Transfusion Medicine, Leiden, Germany

INS-V10

Blood utilisation in the UK and internationally

*J.P. Wallis

Freeman Hospital, Newcastle upon Tyne, Germany

\begin{tabular}{ll}
\hline KARGER & ๑ 2013 S. Karger GmbH, Freiburg \\
Fax +497614520714 & Accessible online at: \\
Information@Karger.com & www.karger.com/tmh \\
www.karger.com &
\end{tabular}


INS-V11

\section{Antibody mediated inhibition of GPIb-VWF interaction as} antithrombotic treatment: Current status

\author{
${ }^{*} H$. Deckmyn', K. Vanhoorelbeke', A.M. Azimzadeh², \\ S.F. de Meyer \\ ${ }^{1}$ Laboratory for Thrombosis Research, IRF-Life Sciences, KU Leuven \\ Kulak, Kortrijk, Belgium \\ ${ }^{2}$ Department of Surgery, University of Maryland School of Medicine, \\ Baltimore, MD, USA
}

Upon injury of the blood vessel, several components of the subendothelium (e.g. collagen, fibronectin, laminin) become exposed, to which circulating platelets can adhere and be activated. In arterial thrombosis, adhering platelets have to withstand high shear forces induced by the fast flowing blood especially in stenotic areas, for which they rely on von Willebrand Factor (VWF). VWF is a large multimeric protein, present a.o. in plasma, that under normal conditions does not interact with its platelet receptor, glycoprotein (GP) Ib, part of the GPIb-IX-V complex. The cryptic GPIb binding site within the VWF-A1 domain becomes accessible, when VWF, bound via its VWF-A3-domain to collagen exposed in the damaged artery, is stretched by the high shear forces.

Based on this understanding, interfering with VWF functions is expected to have an antithrombotic effect specifically targeted to the high shear (stenotic) arterial side, leaving hemostasis in the slower vessels relatively unaffected with anticipated lower bleeding risk. Monoclonal antibodies, proteins and aptamers have been selected that inhibit either the VWF-collagen or the VWF-GPIb interaction. The shear rate dependent activity was proven in in vitro flow chambers with blood pumped at different speeds over a coated collagen surface. But furthermore a strong antithrombotic effect was obtained in several high shear arterial thrombosis models in animals, without prolongation of the bleeding time or increase of blood loss from a standardised incision, and this in stark contrast to other currently used antiplatelet agents. A low risk for bleeding may be particularly useful in the treatment of ischemic stroke and indeed prevention of GPIb-VWF interaction by anti-GPIb or anti-VWF antibodies or by VWF$\mathrm{KO}$ in mice and guinea pig models of ischemic stroke, not only prevented thrombosis and induced reperfusion, but also reduced infarct size and improved functional neurological outcome, without provoking intracranial bleeding. The pathophysiology of thrombotic thrombocytopenic purpura (TTP) can be explained by the absence of active ADAMTS13, leading to ultra-large (UL)-VWF multimers that spontaneously interact with platelets without need for elevated shear stress, resulting in platelet aggregates that occlude small vesicles with consequent organ failure. Preventing the formation of UL-VWF-platelet aggregates therefore is an attractive new treatment strategy and indeed inhibitory anti-VWF antibodies resulted in a rapid recovery of severe thrombocytopenia in a baboon model of acquired TTP. A clinical trial is ongoing to assess whether an anti-VWF nanobody is effective in clinical TTP. Finally, xenotransplantation of porcine organs to man may be of marked benefit. However, although transplantation of genetically-modified "humanized" porcine livers into baboons has extended recipient survival significantly, it nevertheless remains limited due to profound thrombocytopenia. The hypothesis that primate platelet sequestration by porcine liver and the associated thrombocytopenia are mediated by a constitutive interaction between porcine VWF and the primate GPIb receptor, was proven by showing that this interaction can safely be interrupted by an anti-GPIb antibody in a clinically relevant model, resulting in less platelet sequestration during the initial two hours of perfusion.

Inhibition of GPIb-VWF interaction thus provides promising results in animal models of a variety of clinical conditions, eventually revealing new roles as e.g. in reperfusion injury in ischemic stroke. However, invariably in all experiments very little bleeding problems are being reported, which in comparison with clinically available antiplatelet agents, indicates a significant larger therapeutic window for GPIb-VWF inhibitors making this a class of safer antithrombotics.
INS-V12

\section{Immunthrombozytopenie}

${ }^{\star} V$. Kiefel

Universitätsmedizin Rostock, Institut für Transfusionsmedizin, Rostock, Germany

INS-V13

\section{Engineering $\mathrm{T}$ cells for cancer therapy}

${ }^{*}$ C.H. June

Perelman School of Medicine at the University of Pennsylvania Department: Pathology and Laboratory Medicine, Philadelphia/USA, United States

INS-V14

Retrovirus-based vectors for stem cell modifications

${ }^{*}$ A. Schambach

Hannover Medical School, Experimental Hematology, Hannover, Germany

INS-V15

Stem cell gene therapy for pediatric disorders

${ }^{*} H$. Hanenberg

Indiana University, Department of Pediatrics, Indianapolis/USA, Germany

INS-V16

Recent findings on the biochemical and genetic basis of blood group antigens

*J.-P. Cartron

INTS, Paris, France

INS-V17

Granulozyten: Antigene, Antikörper - ein Update

${ }^{*} A$. Reil

DRK-Blutspendedienst West, Labor für Leukozytenimmunologie, Hagen, Germany

INS-V18

Aktueller Stand der Antikörperdiagnostik in der Transplantationsimmunologie

${ }^{*}$ C. Süsal

Inst. für Immunologie INF 305, Abt. Transplantationsimmunologie Heidelberg, Germany

INS-V19

Neue orale Antikoagulantien

${ }^{*}$ S. Konstantinides

Universitätsmedizin Mainz, Centrum für Thrombose und Hämostase, Mainz, Germany 
INS-V20

Transcription 3D

${ }^{*}$ F. Grosveld

Erasmus Medical Center, Stem Cell Institute, Rotterdam/NL, Netherlands

INS-V21

Ex vivo expansion of red blood cells from human stem cells

*I. Dorn

Max-Planck-Institut für molekulare Biomedizin, Abteilung Zell- und Entwicklungsbiologie , Münster, Germany

INS-V22

Insights into red cell Physiology

${ }^{*} N$. Mohandas

New York Blood Center, New York/USA, United States

INS-V23

Emerging Pathogens and Blood Safety: Moving from Reactive to Proactive Responses

${ }^{*}$ M. P. Busch

Blood Systems Research Institute, San Francisco/USA, United States

INS-V24

Reduction of transfusion transmitted bacterial infection: Detection of bacterial contamination of blood components

*J. Dreier

Universitätsklinik der Ruhr-Universität Bochum, Institut für Laboratoriumsund Transfusionsmedizin, Herz- und Diabeteszentrum Nordrhein-

Westfalen, Bad Oeynhausen, Germany

Bacterial contamination of blood components remains the most important infectious risk of blood transfusion. Platelet concentrates (PC) are the cause of the majority of transfusion transmitted bacterial infection (TTBI). A lot of prevention methods including donor selection, optimization of blood collection and manufacturing processes have been introduced to reduce the bacterial hazard. In addition, pathogen reduction technology has been proven to reduce the infectious risk of PCs (Intercept, Cerus; Mirasol, TerumoBCT; Theraflex, MacoPharma).

The second approach is to screen blood components for bacteria. Beside conventional microbiological approaches, several efficient methods able to detect bacterial contamination in platelets are available on the market. They need to be divided into cultivation (incubation) and rapid methods. Bacterial screening of PCs with culture methods (BacT/Alert, Pall eBDS) using an early-sampling strategy with the negative-to-date concept of PC release has been implemented in several transfusion facilities.

Because of the initially very low count of bacteria after donation, a certain small sampling error in application of that strategy remains. Rapid methods are able to perform the diagnosis within a short time. Therefore, they allow postponing of sample drawing, ideally up to the time immediately before transfusion. Bacterial screening methods are likewise an alternative to lower the risk of TTBI. The consensus of several studies to optimize the detection of bacterial contamination is the application of rapid detection methods, such as nucleic acid amplification techniques (16S, 23S rDNA NAT), fluorescence assorted cell sorting (FACS, BactiFlow) or immunological detection methods (PGD, Pan Genera Detection system; BacTx) combined with a late sampling strategy.
Since 2009, the PC shelf life is reduced from 5 to 4 days in Germany to minimize TTBI because most of platelet-related septic complications have been observed with older PCs. Anyhow, in 2009 and 2011 two fatal TTBI cases still occurred, and consequently the question about the shortening of PC shelf-life and the optimal screening strategy was raised again. Improved bacterial detection have decreased, but not resolved the risk of bacterial contamination. The current developments and the challenges that remain to minimize and detect bacterial contamination of platelet products are reviewed.

INS-V25

Prion-ähnliche Mechanismen bei der Alzheimerund Parkinson-Krankheit: Bedeutung für die Transfusionsmedizin?

${ }^{*} R$. Burger

Robert Koch-Institut, Berlin, Germany 


\section{Oral Abstract Session: Blutspende \& Spenderrekrutierung}

\section{SPE-V01}

\section{Motivating blood donors through phone reminders:} Evidence from a long-term randomized evaluation

${ }^{*}$ L. Goette ${ }^{1}$, A. Bruhin 1 , A. Markovic ${ }^{2}$, A. Röthlisberger ${ }^{2}$, R. Buchli², B. Frey ${ }^{2}$

${ }^{1}$ University of Lausanne, Lausanne-Dorigny, Switzerland ${ }^{2}$ Stiftung Zürcher Blutspendedienst SRK, Schlieren, Switzerland

Background: Mobilizing blood donors in times of temporary shortages is a common problem for many blood donation services. It has become more common to use phone calls and reminders to increase turnout among blood donors. Yet, the short-term and long-term effects of such strategies are largely unknown. Phone reminders seem to increase short-term turnout, but nothing is known about its long-term effects. Theory offers little guidance, as theories offer conflicting predictions: some theories predict positive long-term effects of nudging individuals to donate, other predict negative long-term consequences for donor motivation.

Methods: We perform a two-year randomized evaluation that allows us to examine how phone reminders affect donor motivation in the short-run and long-run, and to quantify the extent of heterogeneity in behavioral reactions to the interventions. We estimate a finite-mixture model that allows us to estimate heterogenous treatment effects. Identification is particularly powerful in our application, as we have panel data over several invitation for each donor.

Results: We find strong evidence on behavioral heterogeneity with sharply different behavioral responses. Information criteria show that there are two distinct behavioral types (not more and not less). We find that individuals are of one of two types: Approximately $65 \%$ of all individuals have a low baseline of attending a blood drive, but react positively and strongly to phone reminders: it raises their propensity to donate by approximately $5 \%$-points. It also raises their propensity to donate on the next invitation by almost the same magnitude. Thus, the long-run benefits for this group of individuals are almost twice the short-run benefits. However, the remaining $35 \%$ of donors react differently to the phone reminders: they have a strong response to the reminder and much more likely to show up when reminded. However, It reduces their future tendency to donate significantly.

Conclusion: Our results show that donor motivation is complex and heterogenous. Modern statistical techniques are necessary to identify them. The results have practical implication for the optimal application of phone reminders: the statistical model allows one to predict a donor's type with high accuracy and thus to use the resources in the targeting of the donors optimally.

\section{SPE-V02}

\section{7 years of blood donation by repeat blood donors beyond the age of 68 years}

\section{${ }^{*}$ T. Zeiler', J. Lander-Kox', T. Alt' ${ }^{2}$, R. Kessel'2, J. Bux ${ }^{3}$}

${ }^{1}$ DRK-Blutspendedienst West, Zentrum für Transfusionsmedizin Breitscheid, Ratingen, Germany

${ }^{2}$ DRK-Blutspendedienst Rheinland-Pfalz und Saarland, Bad Kreuznach, Germany

${ }^{3}$ DRK-Blutspendedienst West, Hagen, Germany

Background: In Germany, blood donation by donors older than 68 years was made possible for the first time by the regulations for blood donation, published in 2005. A prospective study on the safety of blood donation by elderly blood donors, initiated by the German Red Cross Blood Service West, was finished in 2009 and demonstrated that blood donation by the elderly is safe. Therefore, from 2010 on, we accepted repeat blood donors older than 68 years for routine blood donation and here we report the results of 7 years of blood donation by elderly blood donors.

Methods: Routine data of blood donations collected at the German Red Cross Blood Service West from years 2006 to 2012, recorded with blood donation software BAS 400, were analyzed.

Results: Since 2006 at the German Red Cross Blood Service West more than 170,000 whole blood donations were provided by donors beyond 68 years. The percentage of blood donations provided by repeat donors older than 68 years increased already during the study to $2.22 \%$ of all blood donations until the end of 2009. It steadily increased since then to $4.15 \%$ in 2012. Donor deferral rates of $7.99 \%$ were below average $(9.47 \%)$ and increased to $10.47 \%$ only in donors older than 71 years. Donor reactions $(0.14-0.18 \%)$ were below the average $(0.39 \%)$ even in donors older than 71 years.

\begin{tabular}{|l|l|l|l|}
\hline \multicolumn{4}{|c|}{ Donor deferral rates and reaction rates in donors beyond the age of 68 (in 2012) } \\
\hline & All blood donors & $69-71$ years & $>71$ years \\
\hline Deferral rates & $9.47 \%$ & $7.99 \%$ & $10.47 \%$ \\
\hline Reaction rates & $0.39 \%$ & $0.18 \%$ & $0.14 \%$ \\
\hline
\end{tabular}

\begin{tabular}{|l|l|l|l|}
\hline \multicolumn{3}{|l|}{ Percentage of blood donations provided by donors beyond the age of 68} \\
\hline Year & & $69-71$ years & $>71$ years \\
\hline 2006 & \multirow{3}{*}{ Study } & $0.71 \%$ & $0 \%$ \\
\hline \multirow{2nn}{2007}{} & $1.36 \%$ & $0.01 \%$ \\
\hline 2008 & $1.90 \%$ & $0.06 \%$ \\
\hline 2009 & $2.07 \%$ & $0.16 \%$ \\
\hline 2010 & \multirow{3}{*}{ Routine } & $2.97 \%$ & $0.63 \%$ \\
\hline 2011 & $2.83 \%$ & $1.05 \%$ \\
\hline 2012 & $2.70 \%$ & $1.45 \%$ \\
\hline
\end{tabular}

Conclusion: Repeat blood donors older than 68 years represent a substantial and increasing part of blood donations. The experience with more than 170,000 whole blood donations by repeat donors beyond 68 years proves the safety of blood donation in this group of repeat donors.

\section{SPE-V03}

\section{Comparison of invasive and non-invasive versus venous methods for haemoglobin screening of blood donors}

\section{S. Ardin', * M. Störmer', S. Radojska', L. Oustianskaia', M. Hahn², B. Gathof'}

${ }^{1}$ Transfusionsmedizin, Uniklinik Köln, Köln, Germany

${ }^{2}$ Institut für Medizinische Statistik, Informatik, und Epidemiologie, Uniklinik Köln, Köln, Germany

Background: To prevent phlebotomy of anaemic individuals and to ensure haemoglobin $(\mathrm{Hb})$ content of the blood units, $\mathrm{Hb}$ screening of blood donors before donation is essential. Hb values are mostly obtained by measurement of capillary blood obtained from fingerprick although the reliability of this method is discussed controversially. Therefore the aim of this study was to evaluate rapid, non-invasive methods for $\mathrm{Hb}$ screening.

Methods: Blood donors were screened for $\mathrm{Hb}$ values in three different trials using three different non-invasive methods in comparison to the established fingerprick method and to values obtained from venous samples on a cell counter (Sysmex) as reference. For non-invasive measurements the NBM200 (OrSense, $\mathrm{n}=120$ ), the Pronto-7 (Masimo, $\mathrm{n}=110$ ) and the Haemospect (MBR Optical Systems $\mathrm{GmbH}, \mathrm{n}=117$ ), were used. Invasive measurement was performed using the CompoLab HB (Fresenius, $\mathrm{n}=339$ ). The non-invasive methods were assessed regarding usability.

Results: On the basis of the results obtained from the first trial using the Haemospect the software needed to be optimized and the trial was repeated. This system got the highest score in usability assessment. Technical failures occurred by using the Pronto-7 due to nail polish, skin colour or light influence. The NBM 200 also showed a high sensitivity against light influence and noticeable lower $\mathrm{Hb}$ values for women than obtained from the Sysmex. Moreover the NBM 200 needed the longest test-time 
between 75 and $115 \mathrm{~s}$. The bias, standard deviation of differences (SDD), and $95 \%$ limits of agreement (LOA) of all methods in comparison to the Sysmex results, estimated by using Bland Altman statistics, are displayed in the table. High differences in the values for the bias are only observable between the Pronto-7 in comparison to the bias of the other two non-invasive methods.

Conclusion: Non-invasive $\mathrm{Hb}$ tests represent an attractive alternative by eliminating pain and reducing infection risk. The main problem for generating reliable results seems to be pre-analytical variability in sampling. Depending on the technology, results were influenced by external factors. Due to the short test-time ( $26 \mathrm{~s}$ ) and low bias in comparison to the venous values, however the Haemospect seemed to have advantages over the other instruments tested. In general, based on the established values all methods are suitable for $\mathrm{Hb}$ measurement.

\begin{tabular}{|c|c|c|c|c|}
\hline & \multicolumn{3}{|c|}{ non-invasive } & \multirow{2}{*}{$\begin{array}{c}\text { invasive } \\
\underset{\text { CompoLab }}{\mathrm{Hb}}\end{array}$} \\
\hline & Haemospect & NBM 200 & Pronto-7 & \\
\hline $\begin{array}{l}\mathrm{N} \\
\text { (donors) }\end{array}$ & 117 & 120 & 110 & 339 \\
\hline bias $[g / d l]$ & $-0,07$ & $-0,12$ & $-0,50$ & $-0,56$ \\
\hline SSD & 1,19 & 1,14 & 0,99 & 0,90 \\
\hline $95 \% \mathrm{LOA}$ & $-2,40$ to 2,27 & $-2,35$ to 2,11 & $-2,44$ to 1,43 & $-2,32$ to 1,21 \\
\hline
\end{tabular}

Fig. 1
SPE-V04

\section{Introduction of a new noninvasive method for Hemoglobin measurement (Haemospect) on mobile donation sites at the Bavarian Red Cross Transfusion service}

${ }^{*}$ M. Wichmann ${ }^{1}$, F. Weinauer ${ }^{2}$

${ }^{1}$ Blutspendedienst des Bayerischen Roten Kreuzes, Produktion, Wiesentheid, Germany

²Blutspendedienst des Bayerischen Roten Kreuzes, Geschäftsführung, München, Germany

Background: Hemoglobin measurement has been well established for years now. Due to some problems by drawing capillary blood samples the results do not comply very well with results received from venous blood samples. Additionally donors do not like being punctured at their fingertip or ear because of pain. Therefore we validated a new method for noninvasive Hemoglobin measurement (Haemospect). After receiving permission by the Paul Ehrlich Institute (PEI) we have recently started to introduce it as a routine method on mobile donation sites and now report about first experiences. Methods: Two mobiles were equipped with a Hemospect. On each donation site voluntary blood donors have been asked to undergo both a capillary and a noninvasive Hemoglobin mesurement. When either of the techniques gave a result close to donation limits $(12,5 \mathrm{~g} / \mathrm{dl}$ for women, $13,5 \mathrm{~g} / \mathrm{dl}$ for men) an additional venous blood sample was drawn and analysed by a Sysmex XS1000i. The venous result was taken as a gold standard to judge, whether admission or rejection of donors by the other two methods was correct (see Results 1).

In order to avoid local influences, the number of venous blood samples was limited to 5 per donation site. After having reached this limit, the donors Hemoglobin continued to be measured by the noninvasive method only. Donor rejection of this unselected population is presented in section Results 2. Staff members were interviewed to receive comments regarding handling, problems, advantages and donor acceptance. 
Results 1: (Table 1 and 2)

It is not surprising that more women than men were selected. Less donors were falsely admitted to donation by the noninvasive method compared to the capillary method contributing to donor safety. In contrast less donors were falsely rejected by the capillary method.

Results 2: (Table 3)

$2,81 \%$ of all donors in an unselected donor population were rejected.

Comments by staff and donors

Though results can be obtained more quickly by capillary testing, the noninvasive method is easy to handle and can be well integrated. Standardization of handling is important to obtain correct results. The usage of Gloves and disinfection can be reduced to a minimum resulting in a lower total process time. The noninvasive method contributes to both donor and staff safety by avoiding blood contact. Nearly all donors prefer the new noninvasive method.

Conclusion: The Bavarian Red Cross Transfusion Service has introduced a new noninvasive method for Hemoglobin measurement (Haemospect) as a routine method on mobile donation sites after having received permission by the Paul Ehrlich Institute (PEI). The new technique contributes both to donor and staff safety due to less falsely permitted donors and avoidance of blood contact. The total donor rejection rate in an unselected donor population is acceptable yet there is still potential for further improvement.

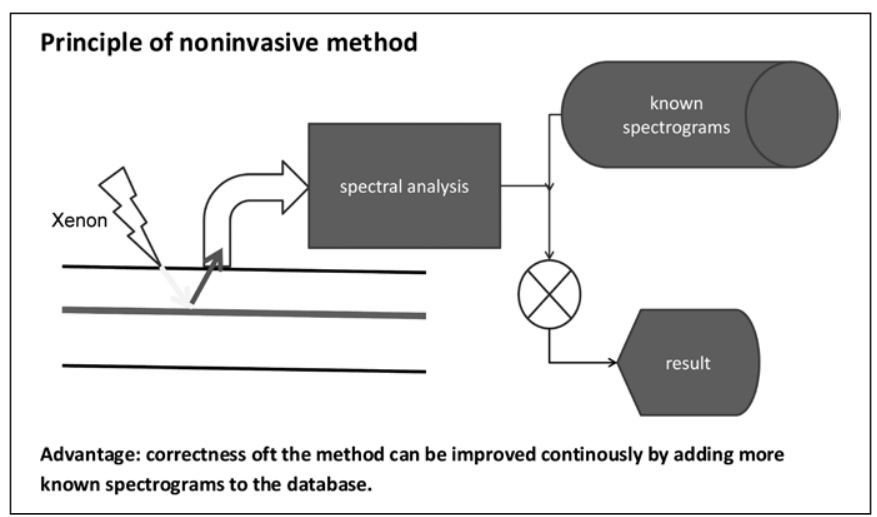

Table 1. Near limit donors involved

\begin{tabular}{|l|l|l|}
\hline men & 64 & $32 \%$ \\
\hline women & 138 & $\mathbf{6 8 \%}$ \\
\hline total & 202 & \\
\hline
\end{tabular}

Table 2. Near limit donors' results

\begin{tabular}{|l|l|l|l|l|}
\hline \multicolumn{3}{|l|}{ Haemospect } & \multicolumn{2}{l|}{ capillary } \\
\hline $\mathrm{n}=202$ & correct & incorrect & correct & incorrect \\
\hline admission & 113 & 38 & 142 & 47 \\
\hline & $55,94 \%$ & $\mathbf{1 8 , 8 1 \%}$ & $70,30 \%$ & $\mathbf{2 3 , 2 7 \%}$ \\
\hline rejection & 20 & 31 & 11 & 2 \\
\hline & $9,90 \%$ & $\mathbf{1 5 , 3 5 \%}$ & $5,45 \%$ & $0,99 \%$ \\
\hline
\end{tabular}

Table 3. Unselected donors' rejection

\begin{tabular}{|l|l|l|l|}
\hline & women & men & total \\
\hline admission & 1710 & 2357 & 4067 \\
& $98,84 \%$ & $98,91 \%$ & $97,16 \%$ \\
\hline rejection & 93 & 26 & 119 \\
& $5,16 \%$ & $1,09 \%$ & $\mathbf{2 , 8 4 \%}$ \\
\hline $\mathrm{n}=$ & 1803 & 2383 & 4186 \\
\hline
\end{tabular}

SPE-V05

\section{The hemoglobin content of red blood cell concentrates depends on the pre-donation hemoglobin detection method}

*A. Sümnig1, T. Thiele ${ }^{1}$, A. Petersmann ${ }^{2}$, A. Greinacher ${ }^{1}$, G. Hron ${ }^{1}$

${ }^{1}$ Institut für Immunologie und Transfusionsmedizin, Abteilung

Transfusionsmedizin, Greifswald, Germany

${ }^{2}$ Institut für klinische Chemie, Greifswald, Germany

Background: Pre-donation hemoglobin $(\mathrm{Hb})$ values need to be above 8.4 $\mathrm{mmol} / \mathrm{l}$ in male and $\geq 7.8 \mathrm{mmol} / 1$ in female donors. In addition, the final $\mathrm{Hb}$ content of a red blood cell concentrate ( $\mathrm{RBC}$ ) has to meet the cut-off of $\geq 40 \mathrm{~g}$ per unit with a minimum level of $38 \mathrm{~g}$ per unit. In this study we aimed to determine the impact of pre-donation $\mathrm{Hb}$ values, assessed by either capillary-, non-invasive, or venous blood measurement on the final $\mathrm{Hb}$ content of RBCs produced by the buffy coat method.

Methods: All included donors were screened for blood donation eligibility according to standard regulations. Capillary-, non-invasive, and venous $\mathrm{Hb}$ measurement was performed in duplicate using a HaemoCue $\mathrm{Hb}$ 301, a Masimo Pronto-7, and a Siemens Advia 2120i, respectively. 553 donors were included into the study. The mean $( \pm \mathrm{SD}) \mathrm{Hb}$ values were $9.1( \pm 0.9) \mathrm{mmol} / \mathrm{l}$ in capillary blood, $8.5( \pm 0.8) \mathrm{mmol} / 1$ by non-invasive measurement, and $8.9( \pm 0.8) \mathrm{mmol} / \mathrm{l}$ in a venous blood sample. The capillary pre-donation $\mathrm{Hb}$ value was significantly higher $(\mathrm{p}<0.001)$ when compared to the venous $\mathrm{Hb}$ levels. In contrast, the non-invasive $\mathrm{Hb}$ value was significantly lower than $\mathrm{Hb}$ level measured in the venous blood sample $(\mathrm{p}<0.001)$. The duplicate measurements did not show any significant differences ( $\mathrm{p}>0.25$ for all comparisons).

Results: The median (min-max) Hb concentration of RBCs produced based on pre-donation capillary $\mathrm{Hb}$ measurement was $51.5 \mathrm{~g}$ (30.9-75.9), whereas the amount of hemoglobin increased to $53.7 \mathrm{~g}$ (32.5-75.9) using pre-donation non-invasive measurement, and $52.7 \mathrm{~g}(32.5-75.9)$ if venous pre-donation measurement was performed. Using the traditional cutoffs for female and male blood donors this translated into $2.2 \%$ of RBCs containing $<38 \mathrm{~g} \mathrm{Hb}$ (capillary method), 1.4\% of RBCs containing $<38$ $\mathrm{g} \mathrm{Hb}$ (non-invasive), and $1.1 \%$ of RBCs containing $<38 \mathrm{~g} \mathrm{Hb}$ (venous method). Therefore, we suggest basing the $\mathrm{Hb}$ cut-off for blood donation eligibility on standards established for each measurement method used to ensure sufficient $\mathrm{Hb}$ levels in produced RBCs.

Conclusion: The $\mathrm{Hb}$ values assessed by using different determination methods differs significantly. Increasing the $\mathrm{Hb}$ cut-off for female donors to $8.4 \mathrm{mmol} / 1$ for the capillary method, and $7.9 \mathrm{mmol} / \mathrm{l}$ for $\mathrm{Hb}$ detected by venous blood analysis without changing the cut-off for male donors resulted in a decrease of RBCs containing less than $38 \mathrm{~g} \mathrm{Hb}$. To ensure standardized $\mathrm{Hb}$ levels of RBCs produced by the buffy coat method, one must take into account which method for $\mathrm{Hb}$ level assessment was chosen and adjust those values to the method of RBC preparation.

SPE-V06

\section{Influence of Iron Substitution with a Standard Drug vs a Food Supplement on Blood donation in repeated Blood Donors}

${ }^{\star}$ F. Stötzer ${ }^{1}$, S. Schneider ${ }^{2}$, G. Metzgeroth ${ }^{3}$, J. Brade ${ }^{4}$, M. MüllerSteinhardt ${ }^{1}$, H. Klüter ${ }^{1}$

11 Institute for Transfusion Medicine and Immunology, Red Cross Blood Service Baden-Württemberg - Hessen, Medical Faculty Mannh, Mannheim, Germany

${ }^{2} 2$ Institute for Clinical Chemistry, University Medicine Mannheim, Germany ${ }^{3}$ Department of Oncology and Haematology, Internal Medicine III, University Medicine Mannheim, Germany

${ }^{4}$ Department for Medical Statistics, Medical Faculty Mannheim, Heidelberg University, Germany

Background: Lack of iron is a well-known problem amongst blood donors who donate blood on a regular basis. Especially motivated blood donors who donate frequently, according to the national guidelines, should 
receive a controlled iron substitution. The kind and volume of the iron replacement for healthy blood donors still is subject to discussion with respect to tolerance, safety and efficacy.

Methods: We performed a prospective randomized study that compared the drug used for iron replacement at the blood service (50 mg elementary iron per day) to a food supplement (vitamins plus $20 \mathrm{mg}$ elementary iron per day) in an equivalent cumulative dose of $1200 \mathrm{mg}$ oral iron and to a third group taking $100 \mathrm{mg}$ ascorbic acid over an observation period of two years. Beside safety and tolerance parameters recorded by a donor questionnaire at each visit in a stepwise rating from fully agree (1) to absolutely not applicable (5), the effect on the blood donation commitment was analysed. Laboratory parameters, venous $\mathrm{Hb}$, the mean corpuscular volume (MCV), ferritin and zink protoporphyrine (ZPP) were monitored over an observation period of at least 18 months in all three groups.

Results: Group 1 incorporated 50 donors $(n=50)$ with total number 325 donations, group 2 incorporated 46 donors $(n=46)$ with 314 donations and group 3 incorporated also 46 donors $(n=46)$ with a total of 224 donations. Donor reporting and interview on the day of a donation showed a high compliance in all three groups. Answering the donor questionnaire, all three treatments were well tolerated by the donors. When actively questioned, stomach-ache, diarrhea, constipation, headache etc. were reported by $29 \%$ of the donors in the $50 \mathrm{mg}$ group vs $40 \%$ in the $20 \mathrm{mg}$ group but $9 \%$ in the non-iron treatment group. All symptoms reported were mainly gastrointestinal. The results revealed that iron treatment (Group 1: 1200 $\mathrm{mg}$ iron, $24 \times 50 \mathrm{mg} \mathrm{Fe}$, Plastufer ${ }^{\mathbb{B}}$ mite; Group 2: $1200 \mathrm{mg}$ iron, $60 \times 20$ mg Fe, NBV Vital Kapseln ${ }^{\circledR}$ ) lead to a marked reduction of deferrals for low $\mathrm{Hb}$ in both groups in comparison to the control group 3.

Conclusion: In iron treatment group 1, the deferral rate for low $\mathrm{Hb}$ level was $8 \%$ (i.e. 4 donors) in comparison to group 2 where the deferral rate was $4 \%$ (i.e. 2 donors). In group3, the non iron treatment group, the deferral rate for low $\mathrm{Hb}$ levels was $30 \%$ (i.e. 14 donors). The effect went along with a rise in donations achieved (more than $30 \%$ in both iron groups). Notably supplement with only $20 \mathrm{mg} \mathrm{Fe}$ over a longer period was not inferior. This result is of great importance as the replacement with $20 \mathrm{mg} \mathrm{Fe}$ is regarded as food supplementation. A better tolerance of the low dose iron replacement could not be demonstrated.

\section{SPE-V07 \\ Previous donations critically determine the acceptance of direct questions about sexual risk behavior}

\author{
${ }^{*}$ B. Wistal, D. Anhalt, C. Dennin, M. Lach, T.H. Müller \\ DRK-BSD NSTOB, Springe, Germany
}

Background: A new donor questionnaire has been developed as a standard for nationwide use in Germany. Several questions address sexual risk behavior in an open direct manner. We performed a study to investigate the donors' perception of the new questionnaire and to analyze its relation to socio-demographic parameters.

Methods: The new questionnaire was used for all whole blood donations collected by two mobile teams in Northern Germany over a period of 3 months. Potential donors were kindly asked to respond to six standardized questions related to the donors' perception of the questionnaire directly after its completion: question number $4(\mathrm{Q} \# 4)$ assessed if any questions were perceived as being awkward or problematic.

Results: The new questionnaire was completed by 5046 donors. Out of these only $5.5 \%(\mathrm{n}=275)$ did not provide an answer to $\mathrm{Q} \# 4$. The majority of $66.7 \%(n=3365)$ did not perceive problems with the questionnaire in contrast to $27.9 \%(n=1406)$ who did. The mean proportion of positive responders to $\mathrm{Q} \# 4$ varied slightly with the age range of the donors: $<30$ years $29 \% ;<40$ yrs $32 \% ;<50$ yrs $35 \% ;<60$ yrs $29 \%$; $>60$ yrs $26 \%$. The proportion of positive answers to $\mathrm{Q} \# 4$ are summarized in the following table in relation to additional variables (Table 1).

Significant differences in the positive response rates to Q\#4 were observed for the frequency of donations $(\mathrm{p}<0.0001)$ and age $(\mathrm{p}<0.0001)$ but not gender $(\mathrm{p}=0.08)$ in a model of logistic regression. 
Conclusion:The number of previous donations appears to be the pivotal determinant for a spontaneous irritation of donors by introducing questions directly assessing their sexual risk behavior.

Table 1

\begin{tabular}{|ccccc|}
\hline \# of donations & years of age & gender & $\mathrm{N}$ & \% positive Q\#4 \\
$\leq 10$ & $\leq 40$ & male & 264 & 19.3 \\
$\leq 10$ & $\leq 40$ & female & 352 & 21.6 \\
$\leq 10$ & $>40$ & male & 289 & 16.3 \\
$\leq 10$ & $>40$ & female & 350 & 16.6 \\
$>10$ & $\leq 40$ & male & 394 & 39.3 \\
$>10$ & $\leq 40$ & female & 262 & 38.2 \\
$>10$ & $>40$ & male & 1821 & 28.3 \\
$>10$ & $>40$ & female & 1165 & 32.1 \\
\hline
\end{tabular}

\section{SPE-V08}

\section{Positive psychotropic effects of blood donation are} frequent and potentially important for donor management

\section{${ }^{*}$ M. Esefeld 1 , A. Sümnig ${ }^{1}$, H.- J. Grabe ${ }^{2}$, A. Greinacher ${ }^{1}$}

${ }^{1}$ Institut für Immunologie und Transfusionsmedizin, Abteilung Transfusionsmedizin, Greifswald, Germany

${ }^{2}$ Klinik und Poliklinik für Psychiatrie und Psychotherapie, Greifswald, Germany

Background: Positive psychotropic effects after blood donation like "increased well-being" or "feeling satisfied after donation" have been reported in previous studies. Improved psychology being may be of importance for recruitment strategies for blood donors and to maintain donors. Understanding the biological mechanisms underlying this phenomenon might also help to comprehend the biology of mood regulation. In this study we investigated the kinetics and dynamics of the psychotropic status of blood donors over 8 weeks after blood donation.

Methods: This prospective cohort study consisted of two parts: 1) a qualitative semi-structured interview assessing the psychological and physiological being after blood donation, enrolling 42 whole blood donors (female $\mathrm{N}=24$; male $\mathrm{N}=18$ ) and 2) a questionnaire-based assessment including 7 different items (see results) enrolling 100 whole blood donors (female $\mathrm{N}=47$; male $\mathrm{N}=53$ ). All items were rated on a numeric scale. The questionnaire was administered $15 \mathrm{~min}$ before blood donation, directly during the donation process and then $15 \mathrm{~min}, 6 \mathrm{~h}, 24 \mathrm{~h}, 72 \mathrm{~h}, 1$ week and 8 weeks after donation. A standardized assessment of socio-demographic data, laboratory and physical parameters (e.g. blood pressure, hemoglobin, BMI) was performed prior to donation. Both parts of the study were conducted at the Greifswald blood donation center.

Results: During phase 1, seven psychotropic effects were frequently indicated by blood donors (mood, concentration, satisfaction, resilience, initiative, physical well-being and energy level). With the aid of those 7 dimensions the items for the standardized questionnaire of phase 2 were developed. These items were summarized to the de-novo well-being (WB) scores. Based on a discriminant analysis of the WB scores, subjects were stratified into 2 groups. The first group $(\mathrm{N}=44$, responders) showed an increase in the WB scores after blood donation, while the second group $(\mathrm{N}=56$, non-responders) did not $(\mathrm{p}<0.001)$. The WB scores of responders increased mainly during a period of $24 \mathrm{~h}$ to 1 week after blood donation ( $\mathrm{p}$ $<0.001$ ). Blood donors with hypertension showed significant lower WB scores $15 \mathrm{~min}$ before donation in relation to normotonic blood donors $(\mathrm{p}<$ $0.05)$. Neither time of donation nor obesity were significantly associated with changes in WB scores.

Conclusion: Almost half of the blood donors reported positive psychotropic effects after blood donation, resulting in increased WB scores that lasted for up to one week. We are now aiming to identify the underlying biological mechanisms.
Fig. 1

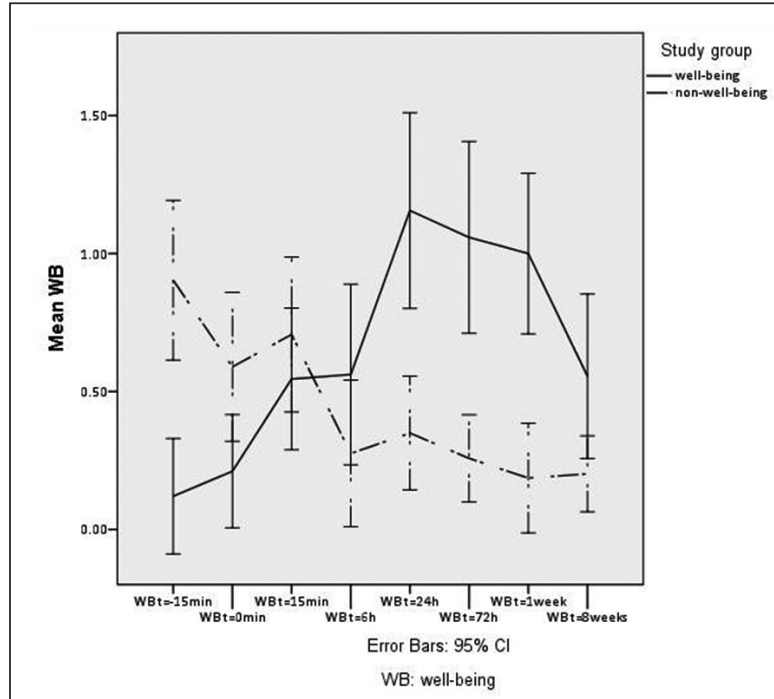

Median well-being (WB) of blood-donors over a time of 8 weeks. The responder group showed a significantly higher WB score compared to the non-responder group at $24 \mathrm{~h}$; $72 \mathrm{~h} ; 1$ week and 8 weeks after blood donation $(p<0.05)$.

\section{SPE-V09 \\ Monitoring of adverse reactions during whole blood donation at the German Red Cross Blood Transfusion Service West in the years 2005 to 2011}

*A. Koppen, K. Nowak, T. Alt, J. Bux

DRK-Blutspendedienst West, Hagen, Germany

Background: Verification of the security of a blood donor is one of the basic principles of a blood transfusion service. This is a precedent condition for the attendance to give blood voluntarily and non-remunerated. Therefore, it is necessary to be conscientious and respectfully in contact with the blood donors also to exclude any hazards with constant efforts. A blood donor, who feels safe and secure especially in rare cases of an unavoidable adverse event, will almost certainly donate blood furthermore. Methods: In this study healthy voluntary and non-remunerated donors ( $>18$ years old) of whole blood donations $(500 \mathrm{ml})$ were included. Specific protocols were developed to documentate which kind of donor is affected (sex, age, first- or repeat donor) and which kind, point in time, treatment etc. of an adverse event occurred. Furthermore is recorded if a donor could go home after the initial treatment, is referred for follow-up treatment to the family physician or is admitted to the hospital. All informations are entered manually into the blood bank software BAS400 and assigned with the unique donor identification number and the individual donation number. Data evaluation is supported by database queries (Crystal Reports) and export of the data to Microsoft Excel for further analysis. Results: About 6.7 million whole blood donations were performed by our blood transfusion service. 19,920 adverse events were evaluated with regard to forecited aspects. The number of adverse events in the last years is comparable and continuously on a low level $(2005: 0.22 \%, 2006: 0.21 \%$, 2007: 0.21\%; 2008: 0.22\%; 2009: 0.28\%, 2010: 0.38\%, 2011: $0.41 \%$ relating to appeared donors). The determined level is under the level reported in the relevant literature. The evaluation shows that female donors suffer two times more often from adverse reactions than male donors, first-time donors 1,5 times more than repeat donors. Approximately $80 \%$ of the symptoms are vasovagal reactions, followed by haematomas $(7 \%)$. Out of 15,266 registered donors with adverse reactions, $0.41 \%$ were rejected from further blood donations. $43 \%$ of this population donated again and $66 \%$ of the repeat donors are female.

Conclusion: The relevance of the issue is in evidence because a lot of countries setup within the scope of hemovigilance a centralized register also for adverse events during blood donation. Thereby increasing infor- 
mation is available, but the comparability has to be done carefully because of complex definitions and subjective grading. Due to the large amount of data collected since 2005 and the constant magnitude of adverse reactions in relation to the collected donations of our blood transfusion service, the statistical data are very meaningful.

\section{Poster: Blutspende \& Spenderrekrutierung}

\section{SPE-P01}

Experience in the use of social networks at the Institute for Transfusion Medicine and Immunohaematology at the University Hospital Magdeburg

\section{${ }^{*}$ S. Schulze', D. Gööck², J. Sieber², M.U. Heim}

${ }^{1}$ Universitätsklinikum Magdeburg A.ö.R, Institut f. Transfusionsmedizin u. Immunhämatologie, Magdeburg, Germany

${ }^{2}$ Webvariants GbR, Magdeburg, Germany

Background: In Germany alone more than 24 million people are currently active on Facebook. For several years the Institute for Transfusion Medicine at the University Hospital Magdeburg is using social networks for public relations. This paper describes the experiences using the Internet portals WebUni and Facebook.

Methods: In the period from 2005 to 2010 the Institute for Transfusion Medicine Magdeburg was cooperating with WebUni, which is a socio-active web portal for students in Magdeburg. Since 2010 our institute has been operating its own page on Facebook. In addition the institute is engaging in the blood donation app on Facebook developed by the company Webvariants GbR for blood establishments.

Results: From 2005 to 2009, the users of WebUni were appealing regularly for blood donation competitions. The number of participants was about 60 people. Since 2010, our institute has been operating its own Facebook page. News, promotions as well as information about blood donation dates are published there. In 2011, the contributions were read by an average of 158 Facebook users within the first 48 hours after release. In 2013 , the number of readers had increased to an average of 355 per post. According to the statistics by Facebook $32 \%$ of visitors to the Facebook page of our institute are between 18 and 24 years, $49 \%$ between 25 and 34 years and $12 \%$ between 35 and 44 years. The blood donation app of the company Webvariants GbR offers a service for Facebook users among blood donors. 300 blood donors of our institute are regularly reporting their blood and plasma donations to the Facebook application.

Conclusion: The use of social networks such as Facebook enables blood establishments to communicate actively with blood donors being part of the respective networks. An advantage is the very rapidly spread out of information to a large group of people. Applications, like the described blood donation app, enable donors to communicate with each other according to the principle: "Do well and talk about it". The users keep their individual donation statistics, receive email reminders of the next donation date and share the information about their completed donations with their Facebook friends.

\section{SPE-P02}

\section{Deferred first-time donors and their return behaviour}

\section{${ }^{*}$ F.-M. Quante, G. Walther-Wenke}

DRK-Blutspendedienst West, Münster, Germany

Background: This study is based on the behaviour of 10,452 prospective first-time donors who were deferred between February and September 2012. The return rate of punctured first-time donors is low. It is even more problematic to motivate prospective first-time donors to donate blood when their first experience with blood donation was being deferred. Explanation of the reason for deferral combined with an incentive might 
be a valuable instrument to influence the return rate of deferred first time donor in a positive way.

Methods: The test group consisted of deferred first-time donors with a temporary deferral and an even donor identification number were sent a personal letter with an enclosed information leaflet and an incentive (sticking plaster case). Donors with a permanent deferral or with a temporary deferral of more than 12 month as well as donors with a deferral based on their weight were excluded. Deferred first-time donors with an odd donor identification number functioned as control group. Donor behaviour has been monitored till the end of the fifth month after deferral took place.

Results: The evaluation of the number of following donations out of both groups showed little difference in their respective behaviour. Samples consisted of 5,226 deferred donors each. 754 (14.43\%) of the test group donated for the first time, whereas $696(13.32 \%)$ of the control group did so. Only the differentiation referring to sex and reason for deferral showed a difference in behaviour. With women there was nearly no reaction to additional contact: after mailing and incentive $14.72 \%$ of the test group and $14.04 \%$ of the control group without further contact donated blood. Of men without additional contact only $11.91 \%$ donated for the first time, whereas additional contact led to an increase to $13.89 \%$. With the most frequent reason for deferral "risk of infection" the return rate was increased with both sexes by the additional contact.

Conclusion: It seems difficult to influence donor behaviour positively. A longer period of observation will show if the return rate of deferred prospective first-time donors can be influenced by the above mentioned instruments.

\section{SPE-P03}

\section{Early detection of metabolic syndrome in blood donors of} Mexico. A pilot study

\section{*J. Rojo ${ }^{1}$, A. Arroyo' 1 I. Sotelo², S. Hernández ${ }^{3}$, M. Rivera ${ }^{4}$}

${ }^{1}$ Centro Nacional de la Transfusión Sanguínea, Dirección General, Mexico City, Mexico

${ }^{2}$ Centro Estatal de la Transfusión Sanguínea de Zacatecas, Mexico,

Mexico

${ }^{3}$ Centro Estatal de la Transfusión Sanguínea de Chihuahua, Mexico, Mexico

${ }^{4}$ Centro Estatal de la Transfusión Sanguínea de Chihuahua, Mexico, Mexico

Background: Obesity is a risk factor for the development of chronic diseases, such as type 2 diabetes and cardiovascular disease. The prevalence of obesity in mexican adults has increased lately. Data from the National Health and Nutrition Survey revealed that $30 \%$ of adults were obese. The identification of cases of obesity or overweight having one or more components of the metabolic syndrome (WHO) is the first step to design a nationwide program for the prevention of type 2 diabetes.

Methods: To find out the number of blood donors from 3 states of Mexico (north, centre and adyacent to Mexico City) with undiagnosed obesity-related metabolic comorbidities for the early detection of metabolic syndrome. Body Mass Index (BMI), gender, weight, height, blood pressure, serum glucose, cholesterol, triglycerides, low and high density lipoproteins and waist-hip ratio were evaluated in 599 donors from the State Centers of Blood Transfusion of Chihuahua (north), Tlaxcala (adjacent with Mexico City) and Zacatecas (centre). Risk factors were considered according to the WHO when 4 of the following were found positive: Systolic pressure $\geq 140 \mathrm{mmHg}$, Diastolic pressure $\geq 90 \mathrm{mmHg}$, Triglycerides $\geq 150 \mathrm{mg} / \mathrm{dL}$, HDL cholesterol: Men

Results: From the total group, 112 were women and 487 male, 165 of them $(27.55 \%)$ presented a risk factor to develop metabolic syndrome. From these, 15 were women and 150 male, with a mean age of 37.4 (18-62) years, BMI 29.6 (22.7-42.32), weight 83.3 Kg (61-119), height $1,68 \mathrm{mts}(1,50-1,90)$, blood pressure $118 / 75 \mathrm{mmHg}(100 / 40-150 / 100)$, serum glucose $103.9 \mathrm{mg} / \mathrm{dL}(71-272)$, cholesterol 206.6 (108-306), triglycerides 273.34 (48-1,005), LDL 130.46 (43-460), HDL 45.58 (23413 ) and waist-hip ratio of $0.97(0.80-1.90) ; 78$ had history of diabetes and only 79 referred physical activity.
Conclusion: The blood banks could be an opportunity area for early detection of metabolic syndrome. These pilot results could be of help to plan and implement a national program in blood banks for early detection of metabolic syndrome in order to defer this population for early attention and education about this problem. Since important differences between the 3 states were found, national further studies should be done in the State Centers of Blood Transfusion, in order to detect differences in the other Mexican States among the population like diets, costumes, life styles, etc.

\section{Fig. 1}

\section{Risk factors for Metabolic Syndrome according to the WHO} Mexican Blood donors from 3 States

\begin{tabular}{|l|c|c|c|c|}
\hline \multicolumn{1}{|c|}{ N: 165} & Minimum & Maximum & Mean & $\begin{array}{c}\text { Std. } \\
\text { Deviation }\end{array}$ \\
\hline Age & 18 & 62 & 37.39 & 9.953 \\
\hline Weigth & 61.0 & 119.0 & 83.830 & 12.0557 \\
\hline Height & 1.5 & 1.9 & 1.682 & 0.0716 \\
\hline BMI & 22.77 & 42.32 & 29.5995 & 3.71894 \\
\hline Sistolicc & 100.00 & 150.00 & 118.9697 & 9.96177 \\
\hline Diastolic & 40.00 & 100.00 & 75.8424 & 7.65407 \\
\hline Glucose & 71.00 & 272.00 & 103.8667 & 25.33039 \\
\hline Colesterol & 108.00 & 306.00 & 206.6606 & 35.35818 \\
\hline HDL & 23.00 & 413.00 & 45.5806 & 31.83578 \\
\hline LDL & 43.00 & 460.00 & 130.4618 & 41.15924 \\
\hline Triglicerides & 48.00 & $1,005.00$ & 273.3455 & 150.94288 \\
\hline Index & 0.80 & 1.90 & 0.9727 & 0.10717 \\
\hline
\end{tabular}

SPE-P04

Prevalence of iron deficiency in Upper Austria - impact of whole blood donation and development of a strategy for detecting and preventing iron depletion in blood donors

\section{*S. Suessner, A. Niklas, C. Gabriel}

Red Cross Transfusion Service of Upper Austria, Speziallabor/ Qualitätskontrolle, Linz, Austria

Background: In the last years much efforts and proceedings have been made in improving safety of blood components as well as to guarantee an adequate blood supply for the aging of our society and for today's advanced medicine. Due to the well-known fact that whole blood donation leads to iron depletion additional measures should be implemented to monitor donor's health. The aims of our study were to identify the prevalence of iron deficiency in Upper Austria and the impact of whole blood donation in this context as well as to introduce a new testing strategy for iron deficiency. Methods: 2594 whole blood donors were enrolled. All data were collected and recorded in the context of routine whole blood donation. Each included donor was in accordance with the Austrian guidelines for blood donor selection and had appropriate haemoglobin levels. Donors were stratified by gender and performed number of whole blood donations.

\begin{tabular}{|l|l|}
\hline Group & Categories \\
\hline I & $\begin{array}{l}\text { First-time donors and donors with no donations in the past } 24 \\
\text { months }\end{array}$ \\
\hline II & Donors with 2 donations in the past 24 months \\
\hline III & Donors with 2 donations in the past 12 months \\
\hline IV & Donors with 3 donations in the past 12 months \\
\hline V & Donors with 4 and more donations in the past 12 months \\
\hline
\end{tabular}

Ferritin, transferrin saturation and several RBC and reticulocyte indices were used for diagnosis of iron depletion. Different iron deficiency stages were defined according the DGHO guidelines. Statistical analysis was conducted with the use of SPSS 20 software.

Results: The prevalence of iron deficiency in the upper Austrian whole blood donor population was $22.8 \%$. In premenopausal women an increased risk for iron deficiency was noticed compared to postmenopausal women $(39.1 \%$ vs $23.6 \%)$. The factor sex pointed to be the strongest pre- 
dictor for iron deficiency ( $p<0.0001)$. Females had an odds ratio of 3.76 compared to males. No statistically significant differences were observed in the distinct age groups. A cut-off value for the parameter Ret-He (reticulocyte haemoglobin equivalent) was also determined in this study and implemented in the new test algorithm.

Conclusion: The implementation of the new suggested test algorithm should contribute to adjust donation frequency (donation intervals) in time and individually in case of iron deficiency. This action improve donor safety, do not cause in higher deferral rates due to low haemoglobin levels in next whole blood donations and so sustain a constant regular donor population.

\section{SPE-P05}

\section{Screening of prostate specific antigen in male voluntary blood donors as a tool of total donor care}

\section{${ }^{\star} R$. Moog, R. Lange, M. Westergom}

plusBlut $\mathrm{GmbH}$, Hennigsdorf, Germany

Background: In times of demographic changes with shrinking number of blood donations on the one hand and an increasing demand of blood components on the other, blood donation services have to re-structure their attitude of donor recruitment.

Prostate cancer occurs in men with increasing age and screening of prostate specific antigen (PSA) is a tool to diagnose the tumour.

The aim of the present study was to implement a health care service for voluntary blood donors as a tool to motivate them to give blood.

Methods: Male blood donors older than 45 years were informed about the possibility of PSA screening. After obtaining informed consent donors samples were screened for PSA by chemiluminescence (DXI, Beckman/ Coulter,Germany). If PSA exceeded $4 \mathrm{ng} / \mathrm{ml}$ the donors were informed by mail and advised to consult an urologist. Feed back was evaluated and a decision on the further donor career was made.

Results: 2013 samples were analysed from January $1^{\text {st }}, 2010$ until December $31^{\text {st }}, 2012$. Elevated PSA levels were found in 62 samples from 49 donors. 4 of these donors were aged 45-50 years, 13 donors were 51 -55 years old. The majority of 20 donors with elevated PSA levels was in range of 56-60 years, followed by 17 donors aged 61-65 years. 10 donors were older that 65 years. Average PSA level was $6.37+3.86 \mathrm{ng} / \mathrm{ml}$. Donors were informed by standard mail to consult an urologist. Donors were followed up for the results of consultation. 3 donors (PSA levels 4.34, 5.60 and $15.70 \mathrm{ng} / \mathrm{ml}$ ) suffered from prostate cancer and underwent operation. 7 donors were lost for follow up. All other donors had no clinical evidence for prostate cancer and were allowed to stay in our donor pool. All donors appreciated our donor care although some were concerned when they received the information about the elevated PSA level.

Conclusion: PSA screening allows to detect prostate cancer at an early stage in otherwise healthy donors and is an adequate tool of donor care. 3 PSApositve donors were operated and a progress of the disease was avoided. Our screening of PSA level was almost welcomed as a total donor care programme.

Fig. 1

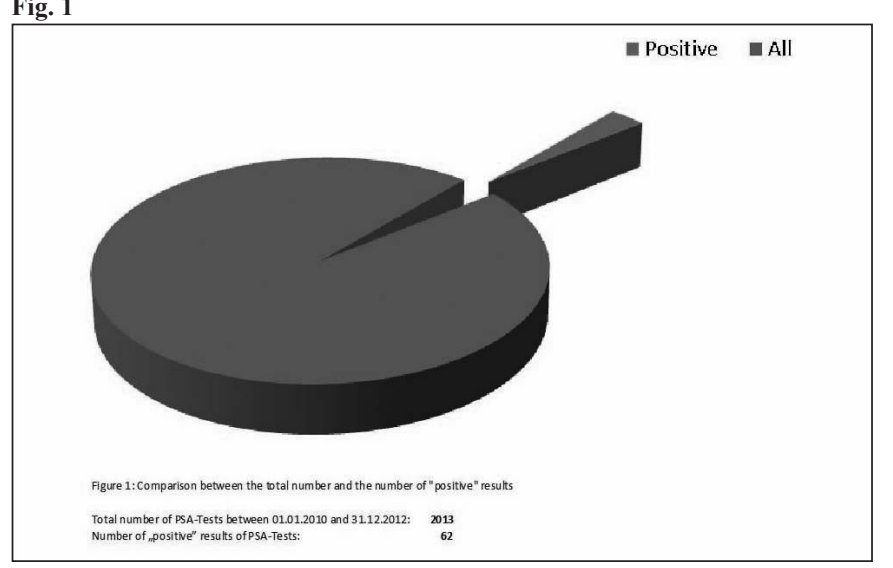

SPE-P06

\section{Lymphopenia in long-term platelet donors}

M. Blechschmidt ${ }^{1}$, M. Kramer ${ }^{2}$, A. Rosner ${ }^{1}, K$. Zimmer ${ }^{1}$, *K. Hölig ${ }^{1}$

${ }^{1}$ Universitätsklinikum Dresden, Transfusionsmedizin, Dresden, Germany

${ }^{2}$ Universitätsklinikum Dresden, Medizinische Klinik I, Dresden, Germany

Background: German Guidelines permit a maximum of 26 platelet donations per donor and year. It is believed, that modern blood cell separators predominantly separate platelets and do not deplete other cell populations, especially leukocytes, to a significant degree. We report on donors developing lymphopenia during long-term plateletpheresis donations.

Methods: At our institution, platelet apheresis is performed with Trima Accel (Version 5.1), Terumo BCT (Lakewood, Co, USA). Donors are allowed to donate a maximum of 26 double platelet concentrates per year. Complete blood counts with differentials are assessed during every $10^{\text {th }}$ platelet donation. A subgroup of our platelet donors attracted our attention with lymphocyte counts remarkably below the normal range. Here we report the characteristics of these donors.

Results: Among 54 long-term platelet donors (donating for more than 1 year, more than 20 donations), we identified 14 donors (13 males, 1 female) with lymphopenia. These donors had donated between 40 and 208 times during a time period of 22-99 months (1,25-2,18 donations/ month). Absolute lymphocyte counts had fallen from initial values of 1,56 $(1,08-1,85) \mathrm{Gpt} / 1$ to $0,96(0,78-1,08) \mathrm{Gpt} / 1(\mathrm{p}<0,001)$. There have been no other abnormal findings in the history of these donors, especially no increased susceptibility for infections. We tested the relationship between the difference in lymphocyte count and the donation frequency. In a statistical model, incorporating the observation period and the number of platelet donations per month, $50 \%$ of the variance of the difference in lymphocyte count could be resolved.

Conclusion: During long-term regular platelet donation in line with the German Guidelines, a substantial proportion of donors $(26 \%)$ shows a considerable decline of lymphocyte counts, depending on the number of donations and the observation period. Analyses of the content of the LRS-chamber of the Trima disposable for platelet collection revealed that a median number of $1.4+0,56 \times 10^{9}$ leukocytes are captured after a double plateletpheresis (Néron et al.). Further investigations are needed to figure out, if these results compromise donor safety.

\section{SPE-P07 \\ Hemoglobin levels and morphological changes in erythrocytes of regular blood donors}

\section{*S. Nowak-Harnau, O. Röhrig, D. Zluhan, B. Luz}

Klinikum Stuttgart, Zentralinstitut f. Transfusionsmedizin u. Blutspendedienst, Stuttgart, Germany

Background: Subclinical iron deficiency in whole blood donors may be a problem for donors accepted for donation while their hemoglobin levels are above the cutoff levels for donation.

Methods: From 01.01.2012 till 31.12.2012 we measured for all donors that did a whole blood donation a complete blood count that was evaluated in our institute with a Cell Dyn blood counter. All donors had screening with finger prick capillary samples using a portable hemoglobinometer. They all had to correspond to the eligibility criteria for donation, wich means a hemoglobin level $\geq 13,5 \mathrm{~g} / \mathrm{dl}$ for men and $\geq 12,5 \mathrm{~g} / \mathrm{dl}$ for women. During the time studied 41178 blood donations had been given.

For 202 blood counts (0,5\%) from 133 donors MCV of the erythrocytes was $<75 \mathrm{fl}$. We analysed the hemoglobin levels the MCV taking into account the number of blood donations within 01.01.2012 till 31.12.2012 or the blood donations given before that time.

Results: Fig. 1

Conclusion: We give 10 tablets ferrous sulfate or ferrous gluconate tablets for all male donors $<14 \mathrm{~g} / \mathrm{dl}$ hemoglobin mesured in the finger prick method and for all female donors with hemoglobin $<13,0 \mathrm{~g} / \mathrm{dl}$. Also donors with $\mathrm{MCV}<80 \mathrm{fl}$ were instructed to take tablets. No datas were 
Fig. 1

\begin{tabular}{|c|c|c|c|c|c|c|c|c|c|c|c|c|}
\hline \multicolumn{7}{|c|}{ Females $(n=49)$} & \multicolumn{6}{|c|}{ Males $(n=84)$} \\
\hline \multicolumn{13}{|c|}{ Number of donations before that time } \\
\hline & $\mathrm{MCV}$ & MCV MIN & MCV MAX & $\mathrm{HB}(\mathrm{G} / \mathrm{DL})$ & HB MIN & HB MAX & MCV & MCV MIN & MCV MAX & HB (G/DL) & HB MIN & HB MAX \\
\hline \multirow[t]{2}{*}{ Median } & 74,2 & 67,8 & 74,9 & 12,7 & 10,6 & 13,4 & 73,3 & 68,2 & 74,8 & 14,6 & 13,8 & 15,8 \\
\hline & $1-9$ & & & & & & $1-9$ & & & & & \\
\hline Median & 74,2 & 69,9 & 74,9 & 12,6 & 11,0 & 14,2 & 74,1 & 67,3 & 74,9 & 13,8 & 12,6 & 16,4 \\
\hline \multirow[t]{2}{*}{ Median } & 73,7 & 70,5 & 74,9 & 12,7 & 11,7 & 14,0 & 74,1 & 68,7 & 74,9 & 14,2 & 12,6 & 16,3 \\
\hline & & & & & & & $>40$ & & & & & \\
\hline \multicolumn{7}{|c|}{ Number of donations within this year } & 73,6 & 71,2 & 74,8 & 14,7 & 12,8 & 16,4 \\
\hline & $\mathrm{MCV}$ & MCV MIN & MCV MAX & HB (G/DL) & HB MIN & HB MAX & MCV & MCV MIN & MCV MAX & HB (G/DL) & HB MIN & HB MAX \\
\hline & 1 & & & & & & 1 & & & & & \\
\hline Median & 74,0 & 70,8 & 74,9 & 12,7 & 11,0 & 13,4 & 73,3 & 68,2 & 74,8 & 13,9 & 13,1 & 15,9 \\
\hline \multirow[t]{2}{*}{ Median } & 74,1 & 67,8 & 74,8 & 12,6 & 10,6 & 14,0 & 74,1 & 67,3 & 74,9 & 14,6 & 12,6 & 16,4 \\
\hline & 4 & & & & & & $4-6$ & & & & & \\
\hline Median & 74,2 & 71,3 & 74,9 & 12,7 & 11,7 & 14,2 & 73,8 & 68,7 & 74,9 & 14,2 & 12,8 & 16,3 \\
\hline
\end{tabular}

available if $\mathrm{MCV}$ value $<75 \mathrm{fl}$ is present in donors with iron deficiency or donors having a hereditary hemoglobinopathy.

With reommendation of iron replacement regarding the hemoglobin values in the finger prick method or marking donors with low MCV levels, we try to prevent our donors from iron deficiency.

\section{SPE-P08 \\ Providing Effective Measures to Prevent Severe Iron \\ Deficiency in Whole Blood Donors: The Roles of Sufficiently Frequent Determination of Serum Ferritin and Adequate Counseling of Donors}

\section{${ }^{*}$ T. Dominka}

Albertinen-Krankenhaus/Albertinen-Haus gGmbH, Institut für Transfusionsmedizin, Hamburg, Germany

Background: The iron loss caused by whole blood donations can contribute to the development of an iron deficiency (ID). It is our aim to protect our donors from this potentially harmful side effect. For many years now, we have tried to achieve this through determining our donors' serum ferritin levels (SF) frequently, providing adequate iron supplementation, adapting the individual donation intervals, and taking sufficient time for giving advice. In 2012 we even intensified this regime and now looked back in order to determine whether this was successful.

Methods: A SF below $30 \mathrm{ng} / \mathrm{ml}$ is internationally regarded as indicating an iron deficiency, which can cause symptoms even without anemia. In September 2012 we adapted our intervention thresholds according to this limit. Now, if it seems likely (judging from SF at former donations, donation frequency, sex, age, body weight etc.), that the current SF is lower than $30 \mathrm{ng} / \mathrm{ml}$ (former threshold: $20 \mathrm{ng} / \mathrm{ml}$ ), the donor is informed and offered iron tablets. If the post-donation review of the laboratory results reveals that the SF is lower than $20 \mathrm{ng} / \mathrm{ml}$ (former threshold: 15 $\mathrm{ng} / \mathrm{ml}$ ) and the donor has not yet got iron tablets, these are mailed. At present, we determine SF at every donation using a turbidometric assay. We now looked through the lab files of the first 240 donors to whom the changed regime was applied in order to determine how their SF values had changed at the time of their next donation.

Results: Of the first 240 donors who had donated in September 2012, until April 2013 a total of $201(83,8 \%)$ had donated at least once more (111 of them female, 90 male), so we could compare the SF at the time of the donations in September $2012\left(1^{\text {st }} \mathrm{SF}\right)$ with the SF at the next following donation $\left(2^{\text {nd }} \mathrm{SF}\right)$.
We found 11 donors $(5,5 \%)$ with $1^{\text {st }} \mathrm{SF}<15 \mathrm{ng} / \mathrm{ml}$ (mean $1^{\text {st }} \mathrm{SF}: 12,27 \pm$ $1,48 \mathrm{ng} / \mathrm{ml}$ ); 2 of these still had a $2^{\text {nd }} \mathrm{SF}<15 \mathrm{ng} / \mathrm{ml}$ (mean $2^{\text {nd }} \mathrm{SF}: 31,0 \pm$ $20,03 \mathrm{ng} / \mathrm{ml})$.

$1^{\text {st }} \mathrm{SF}<20 \mathrm{ng} / \mathrm{ml}: 30$ donors $(14,9 \%)$ including the donors in the group above; mean $1^{\text {st }} \mathrm{SF}: 15,67 \pm 2,89 \mathrm{ng} / \mathrm{ml}$. 7 of these still had a $2^{\text {nd }} \mathrm{SF}<20$ $\mathrm{ng} / \mathrm{ml}$; mean $2^{\text {nd }} \mathrm{SF}: 30,43 \pm 16,22 \mathrm{ng} / \mathrm{ml}$.

$1^{\text {st }} \mathrm{SF}<25 \mathrm{ng} / \mathrm{ml}: 62$ donors $(30,8 \%)$ including the donors in the groups above; mean $1^{\text {st }} \mathrm{SF}: 18,69 \pm 3,7 \mathrm{ng} / \mathrm{ml}$. 19 of these still had a $2^{\text {nd }} \mathrm{SF}<25$ $\mathrm{ng} / \mathrm{ml}$; mean $2^{\text {nd }} \mathrm{SF}: 30,04 \pm 15,7 \mathrm{ng} / \mathrm{ml}$.

$1^{\text {st }} \mathrm{SF}<30 \mathrm{ng} / \mathrm{ml}: 85$ donors $(42,3 \%)$ including the donors in the groups above; mean $1^{\text {st }} \mathrm{SF}: 20,93 \pm 4,82 \mathrm{ng} / \mathrm{ml}$. 51 of these still had a $2^{\text {nd }} \mathrm{SF}<30$ $\mathrm{ng} / \mathrm{ml}$; mean $2^{\text {nd }} \mathrm{SF}: 29,33 \pm 14,99 \mathrm{ng} / \mathrm{ml}$.

Conclusion: On the whole these data demonstrate that our program to prevent severe iron deficiency (ID) is effective. The numbers of donors who remained in their respective low SF groups were markedly reduced and the mean SF showed a significant increase, which applies in particular to the donors with initially very low SF who needed to improve their iron stores most urgently. We feel that a selective and well-timed prophylaxis of ID is not possible without knowing about the current serum ferritin level. A personal counseling, with the objective to find the most suitable measures the donor should take to replenish his or her iron stores, is equally important.

\section{SPE-P09}

\section{Transcutaneous, non-invasive methods of determining} hemoglobin concentrations

\section{*H. Jungmann, M. Schietzel}

MBR Optical Systems GmbH, Wuppertal, Germany

Introduction: Transcutaneous, non-invasive methods of determining hemoglobin concentrations offer a very promising alternative to bloodbased methods. Non-invasive technologies are, however, significantly influenced by physiological and environmental factors. Being able to perform valid determinations of concentration requires knowledge of how these factors affect the measurements so that suitable methods can be used to minimize that influence:

Background:The following physiological factors influence non-invasive $\mathrm{Hb}$ measurement: vascular structure, microcirculation, vasomotion, skin structure, pigmentation, melanin concentration, disorders such as Raynaud's phenomenon, medication. The following environmental factors can also influence non-invasive $\mathrm{Hb}$ measurement: temperature, sitting position (changes in blood flow during measurement), movement during measurement, positioning of the optical sensor. 
Material and Methods: Studies among 349 blood donors have measured the effect of the differences in microcirculation between men and women. Subject to the same conditions, a non-invasive $\mathrm{Hb}$ hemoglobin meter and a laboratory analyzer were used. The effect of moving the arm during $\mathrm{Hb}$ measurement was also investigated.

Results: The finger pad of the right middle finger was used as the measuring site. 124 donor measurements were used to determine the influence of temperature fluctuation on the non-invasive determination of $\mathrm{Hb}$ concentrations. The blood-based reference system may constitute another source of error in non-invasive $\mathrm{Hb}$ measurement. For this purpose $\mathrm{Hb}$ concentrations of 78 donors were determined subject to the same conditions and using two different laboratory instruments (Coulter Counter, Sysmex). Furthermore, comparative measurements and results from 843 donor measurements were presented. Here, a laboratory system was used as reference to determine $\mathrm{Hb}$ concentrations. In addition, a non-invasive measuring system (haemospect) and a minimally invasive measuring system (Hemocue) were used for the measurements.

Conclusion: There are highly significant differences in the relevant parameters - tissue $\mathrm{Hb}$, skin concentration and $\mathrm{Hb}$ distribution - between men and women. Arm movement clearly influences the readings. However, temperature fluctuation during measurement has a marginal influence on the results. If the temperature fluctuates greatly, its influence increases significantly. When using two different reference instruments, the bloodbased $\mathrm{Hb}$ concentrations determined in parallel may differ by up to $1.5 \mathrm{~g}$ / $\mathrm{dL}$. The comparative measurements between haemospect and Hemocue yielded similar results for both systems.

\section{SPE-P10}

\section{Use of the 'uniform' questionnaire for blood donation in} Germany

\section{B.S. Gathof, A. Sauermann, *S. Radojska}

Uniklinik Köln, Transfusionsmedizin, Köln, Germany

Background: For safety of blood donation the detailed evaluation of risks of the donor with respect to his health and potential risk behaviour is necessary. In Germany a group of experts for the Arbeitskreis Blut of the Robert Koch Institute had developed a questionnaire, which, after scientific testing, was recommended to be used by all blood donation centers Some of the questions were debated by donors and blood centers.

Methods: The use of the uniform questionnaire was looked up in the information available in the internet platforms of the blood donation services in Germany. Their conformity with the recommended questionnaire was evaluated.

Results: In May 2013 internet platforms of 66 German blood donation services were evaluated. Of these were 6 from the German / Bavarian Red Cross, 57 hospital or community associated, 2 private organisations. In total 9 questionnaires were available, 8 could be printed out. 4 questionnaires were identical with the recommended version, thereof 1 with minor modifications, and 5 questionnaires showed differences. The differences were amongst others in questions $16-18$ in 4 of 5 questionnaires leading to imprecise answers.

The donor questionnaire should be part of the donor marketing. Therefore its integration in the website of donor centres is desirable. If the majority of donor centres choose not to show this information this indicates a communication problem.

Conclusion: In this small evaluation only about $13.4 \%$ percent of the questionnaires could be evaluated. This study showed that the "uniform'questionnaire is not used by the majority of the blood centers in Germany. A new discussion seems necessary to obtain a recommendation, which could be integrated in the individual donor history by all blood centres in Germany.

\section{Oral Abstract Session: Hämostaseologie}

\section{HÄM-V01 \\ Immunization against protein/heparin complexes in pediatric patients undergoing cardiac surgery}

${ }^{*}$ R. Jouni' ${ }^{1}$, H. Zöllner ${ }^{1}$, J. Bagemühl' ${ }^{1}$, U. Strobel ${ }^{1}$, A. Koster ${ }^{2}$, J. Amiral ${ }^{3}$, A. Greinacher ${ }^{1}$, T. Bakchoul ${ }^{1}$

${ }^{1}$ Institute for Immunology und Transfusion Medicine, Ernst-Moritz-Arndt University, Greifswald, Germany

${ }^{2}$ German Heart Institute (DHZB), the Department of Anesthesiology, Berlin, Germany

${ }^{3}$ Hyphen BioMed, Paris, France, Germany

Background: The polyanion heparin binds charge related to positively charged proteins such as platelet factor 4 (PF4) and protamine (PRT). Antibodies against PF4/heparin and protamine/heparin complexes are frequently formed in adults after cardiac surgery during which protamine is given to neutralize heparin. In adult patients undergoing cardiac surgery we recently found anti-PF4/heparin and anti-PRT/heparin IgG antibodies in $52.5 \%$ and $26.6 \%$ of patients, respectively.

Aim: To investigate the incidence of PF4/heparin and PRT/heparin antibodies in pediatric patients undergoing cardiac surgery.

Methods: A prospective cohort of 101 pediatric patients (median age: 4 months, range 1-163 months) undergoing cardiac surgery was investigated. Blood samples were collected in five-day intervals until postoperative day 15. Sera were tested for PF4/heparin- and PRT/heparin antibodies using in-house immunoassay (EIA) that separately detects IgG/M antibodies. Platelet activation capability was assessed using the heparin induced platelet aggregation assay (HIPA).

Results: 40/101 (39.6\%) patients tested positive for anti-PF4/heparin antibodies of any class (IgG: $n=4, \operatorname{IgM}, n=27$, and both: $n=9$ ), among whom 2/101 (2.0\%) were platelet activating. Anti-PRT/heparin antibodies were detectable in 18/101 (17.8\%) pediatric patients of any class (IgG: $n=4$, IgM: $n=13$, and both: $n=1)$. Three patients $(3.0 \%)$ had platelet-activating anti-PRT/heparin antibodies at the time of surgery, when protamine and heparin is given. None of these patients developed postsurgery thrombocytopenia or thromboembolic complications.

Conclusion: The IgG antibody responses towards PF4/heparin- or PRT/ heparin-complexes are less frequent in children compared to adults. This indicates that this immune response is driven by B-cells which depend on cofactors which are more present in adults than in children.

\section{HÄM-V02 \\ Increased activation of the coagulation cascade is not a countermeasure to prevent bleeding in patients with immune thrombocytopenic purpura}

\section{${ }^{*} H$. Rühl', B. Pötzsch ${ }^{1}$, J. Oldenburg ${ }^{1}$, G. Bein ${ }^{2}$, U. J. Sachs ${ }^{2}$}

${ }^{1}$ Universitätsklinikum Bonn, Institut für Experimentelle Hämatologie und Transfusionsmedizin, Bonn, Germany

2Justus-Liebig-Universität Gießen, Institut für Klinische Immunologie und Transfusionsmedizin, Gießen, Germany

Background: The bleeding risk in patients with immune thrombocytopenic purpura (ITP) does not always correlate with the platelet count. Coagulation factor concentrates have been used successfully in cases of severe haemorrhage in ITP and have been shown to at least partially correct the ITP-induced coagulopathy in vitro. Therefore an increased activation status of the plasmatic coagulation could contribute to the variability of the bleeding phenotype in ITP. Methods: To evaluate the activation status of the clotting cascade we simultaneously measured plasma levels of thrombin and of the thrombin-activated endogenous anticoagulant activated protein C (APC) in plasma samples of patients with ITP using a recently developed oligonucleotide-enzyme capture assay (OECA) platform. The diagnosis of ITP was confirmed by the monoclonal antibody immobilization of platelet antigen assay (MAIPA). In addition to the measurement of plasma levels of thrombin and APC, pro- 
thrombin fragment $1+2(\mathrm{~F} 1+2)$, thrombin-antithrombin (TAT)-complexes, and antithrombin activity were measured using routinely established assays. Results: Plasma samples of 10 patients ( 7 females) with MAIPA-confirmed ITP (mean age, range: 55, 24-75 years) were available for analysis. Thrombin and APC levels above the respective limits of detection $(0.017 \mathrm{ng} / \mathrm{ml}$ for thrombin, $0.022 \mathrm{ng} / \mathrm{ml}$ for APC) were not observed. The majority of samples showed plasma levels of F1+2 and TAT-complexes within the reference ranges of our laboratory of $£ 0.34 \mathrm{nmol} / 1$ for F1+2 and $0.1-3.9 \mathrm{ng} / \mathrm{ml}$ for TAT-complexes. In one patient markedly increased TAT-complexes of $11.6 \mathrm{ng} / \mathrm{ml}$ were observed.

Conclusion: Using ultrasensitive detection methods detectable plasma levels of thrombin and APC were not observed in ITP patients. Our data suggest that an increased activation status of the plasmatic coagulation is not a frequent finding in ITP.

\section{HÄM-V03 \\ PTGS1 compound heterozygosity impairs gene expression and platelet aggregation and is associated with severe bleeding complications}

${ }^{*}$ P. Bugert ${ }^{1}$, E. Yagmur ${ }^{2}$, R. Weiskirchen ${ }^{3}$, H. Klüter ${ }^{1}$, A. Schedel ${ }^{1}$

${ }^{1}$ DRK-Blutspendedienst Baden-Württemberg - Hessen, Institut für Transfusionsmedizin und Immunologie, Mannheim, Germany ${ }^{2}$ Medical Care Center Dr. Stein and Colleagues, Mönchengladbach, Germany ${ }^{3}$ Institute of Clinical Chemistry and Pathobiochemistry, Aachen, Germany

Background: The prostaglandin endoperoxide synthase (PTGS1, COX1) catalyze the conversion of arachidonic acid (AA) into thromboxane $\mathrm{A}_{2}$ $\left(\mathrm{TxA}_{2}\right)$. Polymorphisms of genes involved in AA metabolism are potential modifiers in platelet function. The Aspirin-like defect (ALD) is an inherited platelet function disorder that involves the AA pathway, however, the molecular basis remains unknown. A patient with postoperative recurrent disseminated bleeding showed strongly deminished platelet aggregation response on AA indicating an ALD. Our study describes the molecular genetic investigation of the AA metabolism genes in this patient.

Methods: Platelet function was analyzed by light transmission and whole blood impedance aggregometry using different agonists including ADP, AA and U46619. Molecular genetic investigation included exon re-sequencing of the PTGS1, TBXAS1 and TBXA2R genes. In addition, gene transcripts were quantified in platelet RNA using realtime-PCR (qRT-PCR). PTGS1 protein expression was further investigated by Western blot analysis.

Results: AA-induced platelet aggregation response was significantly decreased, whereas platelet aggregation induced by U46619 as indicator for TxA -receptor function was unaffected. The TBXAS1 and TBXA2R genes revealed regular DNA sequences, whereas, the coding region of the PTGS1 gene of the patient was compound heterozygous (c.22C>T; c. $50 \mathrm{C}>\mathrm{T}$ ). We found that PTGS1 mRNA and protein expression in the patient's platelets was significantly decreased compared to unaffected normal controls (5-fold) or single heterozygotes (2-fold).

Conclusion: Compound heterozygosity in the 5' region of PTGS1 impairs gene expression and is associated with a phenotype of severe bleeding complication. Therefore, surgeons and anaesthesiologists should be aware of risks, which go with PTGS1 polymorphisms prior elective surgery.

\section{HÄM-V04 Methyprednisolone is a possible cause of drug-induced
immune thrombocytopenia (DIT)}

\section{${ }^{*}$ V. Kiefel', A. Winkelmann², S. Kranz ${ }^{2}$, I. Schönberner-Richter ${ }^{1}$,} U. Walter

${ }^{1}$ Institut für Transfusionmedizin, Rostock, Germany

${ }^{2}$ Universitätsmedizin Rostock, Klinik und Poliklinik für Neurologie, Rostock, Germany

Background: Acute severe thrombocytopenia is sometimes caused by drug-dependent antibodies (ddab). Ddab represent a unique type of platelet autoantibodies binding to platelet glycoproteins in the presence of the offending drug only. Prototypic substances implicated in DIT are quinine and quinidine, however, ddab against platelets reacting with many other substances have been described. Diagnosis is usually made by the assessment of the medical history and it can be confirmed by serologic tests for ddab.

Patient/Methods: A 23-year-old female patient with multiple sclerosis developed acute, severe thrombocytopenia (minimal platelet count $\left.1 \times 10^{\wedge} 9 / 1\right)$. A tentative diagnosis of DIT was made and blood samples were screened for ddab using an enzyme immunoassay (ELISA) with intact test platelets in suspension: pantoprazole,zopiclone, lorazepan, ranitidine, dimetindene, fingolimod, paracetamol and methylprednisolone.

Results: Of the drugs screened with the patient's serum, only methylprednisolone (MP) induced binding of ddab to platelets. The antibody was shown to be specific for the glycoprotein (GP) IIb/IIIa complex using the MAIPA assay. The patient had received four treatments with high doses of MP in the 14 months before this last treatment responsible for DIT. MP was discontinued and platelet counts rose to $178 \times 10^{\wedge} 9 / 1$ four days later. The ddab did not react with platelets in the presence of dexamethasone and the patient was treated with dexamethasone approximately four weeks later without effects on the platelet count.

Conclusion: To our knowledge this is the second case of DIT caused by a methylprednisolone-dependent antibody identified by immunological methods. Also the first case (Royer B et al., Blood 2010;115:5431) was a patient with multiple sclerosis under treatment with MP. These two exciting observations prove that a drug, which occasionally is used for treatment of ITP may itself cause severe immune thrombocytopenia.

\section{HÄM-V05 \\ Neoplasm-induced bleeding in inherited FXIII subunit A deficiency - a case report}

${ }^{*}$ V. Ivaskevicius, N. Marquardt, S. Horneff, C. Klein, G. Goldmann, B. Pötzsch, J. Oldenburg

Institute of experimental Haematology and Transfusion Medicine, Haemophilia Center, Bonn, Germany

Background: Inherited mild factor XIII (FXIII) deficiency belongs to one of the most underdiagnosed bleeding disorders so far. This is, because most patients do not develop bleeding complications in daily life.

Methods: A 64-year-old male without a history of bleeding presented with painful swelling of neck, weight loss, anemia and episodic bleeding from the right tonsil necessitating tonsillectomy. Histologic evaluation revealed epithelioid tumor in a tonsil. Immunohistochemistry showed a strong expression of CD31, co-expression of cytokeratin and no expression of CD34 establishing the diagnosis of cytokeratin-positive epitheloid angiosarcoma.

Blood coagulation status was performed and revealed inconspicuous global tests (prothrombin time, activated partial thromboplastin time, thrombin time), normal levels of single coagulation factors, but significantly (20-fold) elevated d-dimer and decreased FXIII levels (FXIII-activity 35\%, FXIIIA-Ag 16\%). Plasma mixing studies excluded neutralizing antibodies against FXIII. There were no signs of hyperfibrinolysis performing thrombelastography.

Results: A novel heterozygous F13A1 gene nonsense mutation (Glu102X, c.307G>T) was found confirming inherited mild FXIII deficiency. Heterozygous Glu102X mutation was also detected in two further asymptomatic relatives (see figure 1)

For further clinical management the patient was transfused with FXIII-concentrate and showed an adequate response ruling out that the FXIII deficiency was induced by increased turnover. Despite this haemostatic management and antifibrinolytic treatment the patient had to undergo several revisions due to delayed, $\mathrm{Hb}$ relevant bleeding after cervical lymph nodes extirpation. Two chemotherapy cycles with paclitaxel and palliative radiotherapy of the neck area were performed, but the patient died unfortunately two months after diagnosis.

Conclusion: It is a unique case showing combination of a highly aggressive angiosarcoma and presence of inherited FXIII deficiency. It is also a 
rare example demonstrating the necessity of FXIII genotyping despite of highly elevated d-dimers.

Fig. 1

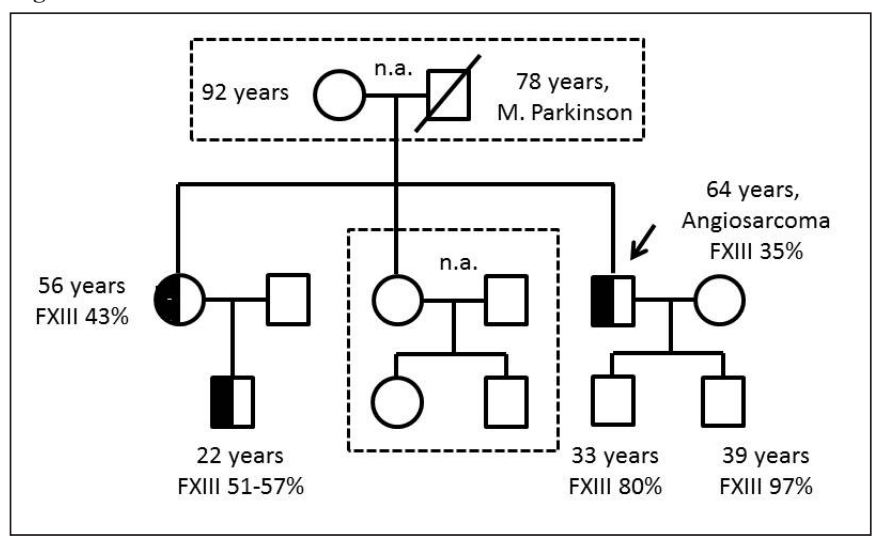

HÄM-V06

The influence of pre-analytical conditions on the analysis of platelet derived microparticles

${ }^{*}$ F.M. Eckstein ${ }^{1}$, W. Xiang ${ }^{2}$,D. R. Weiss ${ }^{1}$, R. Zimmermann ${ }^{1}$, ${ }^{*} E$. Strasser ${ }^{1}$

${ }^{1}$ Universitätsklinikum Erlangen, Transfusionsmedizinische und Hämostaseologische Abteilung, Erlangen, Germany

${ }^{2}$ FAU Erlangen-Nürnberg, Biochemisches Institut, Erlangen, Germany

Background: Microparticles (MP) have recently become a focus of both research and clinical investigations. As pre-analytical conditions frequently remain unpublished, further studies are needed to analyse their impact on MP release.

Methods: This prospective study investigated the effect of sequential storage under three different sets of conditions (fresh; storage at $4{ }^{\circ} \mathrm{C}$ for 24 hours, $\mathrm{SC} 1$; storage at $-70{ }^{\circ} \mathrm{C}$ for $24 \mathrm{~h}, \mathrm{SC} 2$ ) and agitation on platelet-derived MP (PMP) in 11 healthy blood donors (6 male, 5 female). PMP were quantified using flow cytometry (FCM) for analysis of all events positive for both CD41a-PE and Annexin-V-FITC. New developed calibration beads for FCM (size of $0.3-0.9 \mu \mathrm{m}$ ) were applied for FCM. For functional testing a phospholipid-dependent clotting assay (XACT) was used.

Results: PMP concentration increased 1.7-fold in platelet-poor plasma (PPP) under SC1 and further increased 1.6-fold $(\mathrm{p}<0.001)$ under SC2 $(\mathrm{p}=0.005)$. Overall, samples of $\mathrm{SC} 2$ had a 5.5 -fold increased count of large PMP $(0.5-0.9 \mu \mathrm{m})$ compared to baseline. Results in samples of $\mathrm{SC} 2$ ranged from $40.1 \mathrm{~s}$ to $80.3 \mathrm{~s}$ but on average the CT was also shortened compared to the $\mathrm{CT}$ for $\mathrm{SC} 1$ and fresh samples. Additional agitation before PPP preparation reduced the PMP concentration by around $50 \%$ $(\mathrm{p}=0.025) .135 \%$ more small PMP were detected with recently developed calibration beads. Compared to CT (XACT) flow cytometry using Megamix Plus calibration beads is able to reveal significant differences between the analyzed preanalytical conditions.

Conclusion: For standardizing PMP analysis fresh blood samples should be used. Calibration beads for FCM (size of 0.3-0.9 $\mu \mathrm{m}$ ) has shown to be a reliable tool for PMP quantification especially for PMP of smaller sizes up to $300 \mathrm{~nm}$. Agitation of blood samples before PMP analysis should be avoided. The application of XACT is limited for the analysis of preanalytical conditions.

\section{Poster: Hämostaseologie}

HÄM-P01

Therapeutic Immunoglobulins (IVIG) elevate the thrombogenic potential of monocytes and platelets: Possible relevance for thromboembolic complications

${ }^{*} U$. Salge-Bartels ${ }^{1}$, M. Heiden ${ }^{1}$, N. Groß ${ }^{2}$, R. Seitz ${ }^{1}$

${ }^{1}$ Paul-Ehrlich-Institut, Hämatologie \& Transfusionsmedizin, Langen, Germany 2Paul-Ehrlich-Institut, Immunologie, Langen, Germany

Background: Thromboembolic events (TEE) following administration of IVIG were recently reported to be associated with contaminating FXIa (Etscheid M, Vox Sang, 2012). Because of the arterial manifestation of TEE, we studied whether in addition to the FXIa-dependent clotting, IVIG induced the activation of platelets and monocytes.

Methods: Twenty IVIG samples from five manufacturers were tested for a stimulating effect on whole blood clot formation. Additionally, a selection of non- and TEE-associated lots was further analysed using thromboelastography, platelet aggregation, platelets and monocyte adhesion. Induction of tissue factor (TF) surface expression in monocytes and annexin $\mathrm{V}$ binding to platelets was determined by flow cytometry. Western blotting was used to analyse anti-CD154-reactive proteins. Pure FXIa was used for comparison. Results: In whole blood IVIG enhanced the FXIa-initiated clotting. IVIG induced Fc $\gamma$ RII-receptor-dependent TF expression in monocytes. Intensity of TF induction was similar for all IVIG products, and not affected by FXIa. IVIG strengthened ADP and TRAP-6 elicited platelet aggregation. Furthermore, IVIG increased platelet-monocyte adhesion, annexin $\mathrm{V}$ binding to platelet microvesicles, as well as platelet adhesion to IVIG-coated surfaces. The strongest effects were observed with TEE-associated lots. CD154-related proteins were detected in all IVIG products, and CD154-related high molecular weight complexes were particularly found in one TEE-associated IVIG. In platelet aggregation, recombinant sCD154 enhanced aggregate formation and stability.

Conclusion: Our data demonstrate that IVIG modulate platelet and monocyte activation and can thereby affect the haemostatic balance. CD154-related proteins are assumed to be part of the interactions, the mechanism of which needs to be defined in further studies.

\section{HÄM-P02}

\section{Micro RNA Levels Change during Platelet Activation}

${ }^{*}$ B. Stephan ${ }^{1}$, H. Lauer ${ }^{1}$, S. Gries ${ }^{1}$, J.F. Schenk', C. Welter ${ }^{2}$

${ }^{1}$ Universitätsklinikum des Saarlandes, Institut für Klinische Hämostaseologie und Transfusionsmedizin, Homburg, Germany ${ }^{2}$ Medizinische Fakultät der Universität des Saarlandes, Humangenetik, Homburg, Germany

Background: Platelets lack nuclear DNA but harbour many different RNAs and an active protein synthesis. Likely platelets possess post transcriptional regulation and micro-RNAs play a central role in these mechanisms. A larger number of such regulatory RNAs have been identified in platelets. We aimed at analyzing quantitative changes of micro-RNAs during activation of platelets. Methods: 6 apheresis leucocyte-depleted platelet concentrates of healthy blood donors (written consent for examination was obtained) were leucocyte-depleted for a second time. After this second filtration flow cytometry did not detect any leucocytes in the platelet concentrates. Platelet concentrates were divided into two parts and one part was activated with collagen. RNA was isolated from native and activated platelet aliquots. A pre-screening with a micro RNA array was performed and 11 deregulated micro-RNAs were quantified by Northern blot.

Results: Northern blot results indicated upregulation of all $11 \mathrm{mi}$ cro-RNAs in three aliquots, downregulation in 2 aliquots and no deregulation in one aliquot following collagen activation.

Conclusion: Our results indicate that micro-RNA levels in platelets change during platelet activation, albeit interindividual variation appears to exist. 
HÄM-P03

\section{Advanced platelet characterization using dynamic light scattering}

\author{
A. Labrie ${ }^{1}$, J. Leung ${ }^{2}$, G. Bourne ${ }^{1}$, *E. Maurer-Spurej ${ }^{1,2}$ \\ ${ }^{1}$ Lightintegra Technology, Vancouver, Canada, Canada \\ 'University of British Columbia , Centre for Blood Research, Vancouver, \\ Canada, Canada
}

Background: It is estimated that $10-30 \%$ of platelet transfusions fail to achieve a satisfactory patient outcome. The characteristics of donor blood contribute to product quality, and together with processing factors, may influence transfusion outcomes. Current research methods to characterize platelet components are often invasive, time consuming, costly and require highly trained personnel. Thus, a non-invasive, routine test to screen donors and products for platelet quality is still needed.

Methods: ThromboLUX, a non-invasive test based on dynamic light scattering, was used to characterize platelet samples.Small, $100 \mu \mathrm{L}$ samples of platelet concentrates or platelet-rich plasma were loaded into capillaries. The Doppler shift of the light scattered by the suspended particles was analyzed to determine the microparticle content, platelet shape, platelet temperature response and the extent of microaggregation. From this information a Score was calculated and used to determine the effect of different preparations.

Results: Blood donations with lipemic plasma are often discarded because the risk factors are unknown, and objective measures of turbidity are not practical. Using ThromboLUX, a Score was obtained even when the platelet concentrate was visibly lipemic, and it was possible to determine the maximum tolerable lipid concentration. ThromboLUX was further used to detect microparticles (MP) in samples without the need to isolate them from other particles. MP concentrations of donor and product samples were compared and showed differences depending on the preparation technique. Lastly, we employed ThromboLUX for bacterial detection in platelet samples, and found contaminated samples received lower ThromboLUX Scores. Further studies are required to determine the sensitivity of the device for this application.

Conclusion: ThromboLUX can test donor blood samples prior to blood donation to qualify donors. Rapid donor screening could enhance platelet transfusion performance and eliminate the collection and processing of ineffective platelet components. ThromboLUX provides a comprehensive and novel analysis of platelet samples and has the potential as a routine, non-invasive platelet test.

\section{HÄM-P04}

An increased Plasminogen Activator Inhibitor-1 activity and a reduced factor XII activity may reflect an increased risk factor for the development of recurrent venous thrombosis.

\section{${ }^{*}$ L. Bukreeva, B. Hoppe, A. Salama}

Charité-Universitätsmedizin Berlin, Institute of Transfusion Medicine, Campus Virchow-Klinikum, Berlin, Germany

Background: Deep venous thrombosis (DVT) and venous thromboembolism (VTE) occur secondary to a number of hereditary and acquired disorders of hemostasis. In such patients, a widespread screening for thrombophilic risk factors is frequently performed.

Methods: The following parameters were determined in 127 patients with idiopathic and recurrent deep venous thrombosis ( $\geq 2$ incidents) and 485 patients suffering from non-recurring VTE: the activity of antithrombin, protein C, protein S, PTT, D-Dimer, prothrombin time, fibrinogen, factor VIII, factor XI, factor XII, and PAI-1 activity. For these parameters a statistical comparison between both groups has been performed.

Results: Analysis of fibrinolysis system demonstrated a significant higher PAI- 1 activity $(p=0,021)$ and reduced XII activity $(p=0,003)$ in patients with recurrent VTE as compared to those with non-recurring disease.

Conclusion: Reduced factor XII activity and increased PAI-1 activity may represent risk factors in patients with recurrent VTE.
Oral Abstract Session: Herstellung \& Qualitätskontrolle

\section{HQK-V01 \\ Evaluation of in vivo imaging of magnetically labelled blood cells}

${ }^{*} K$. Aurich ${ }^{1}$, T. Berthold ${ }^{1}$, J. Heidel ${ }^{1}$, N. Schubert ${ }^{1}$, J. Kühn², S. Hadlich ${ }^{2}$, A. Greinacher ${ }^{1}$, T. Bakchoul ${ }^{1}$

1 Universitätsmedizin Greifswald, Transfusionsmedizin, Greifswald, Germany ${ }^{2}$ Universitätsmedizin Greifswald, Institut für Diagnostische Radiologie, Greifswald, Germany

Background: Novel blood products require characterization of blood cell integrity and function. Cellular labelling by magnetic nanoparticles may be a promising approach to study kinetics of transfused blood cells in vivo. We recently developed a method to label platelets and granulocytes with Resovist ${ }^{\circledR}$ iron oxide nanoparticles. Here we report on the monitoring of transfused nanoparticle labelled cells in vivo and ex vivo.

Methods: Platelets and granulocytes were labelled with Resovist ${ }^{\circledR}$. The particle location within cells was analyzed by means of electron and fluorescence microscopy. The impact of magnetic labelling on platelet and granulocyte function was tested by flow cytometry (activation marker), aggregometry (platelet aggregation) and granulocyte agglutination test. The ability of MRI based detection was measured using a 7-Tesla MRI scanner. For those studies in vitro phantom measurements of different cell suspensions and in vivo detection studies after subcutaneous injection of cells (NOD-SCID mice) were performed. Further, the NOD/SCID mouse model was used for long term imaging of circulating human cells administered intravenously, and real time images were made. Finally, labelled human granulocytes were re-isolated from mouse blood by magnetic separation and recovery rate was determined by flow cytometry and atomic absorption spectroscopy.

Results: Labelling of platelets and granulocytes with $10 \mathrm{mM}$ Resovist ${ }^{\mathbb{R}}$ resulted in an iron content of $0.8 \mathrm{pg} / \mathrm{platelet}$ and $1.3 \mathrm{pg} / \mathrm{granulocyte}$, respectively. Particles were localized intracellularly with a labelling efficiency of $\sim 50 \%$, which was sufficient for detection and quantification of cells by MRI. Neither platelet nor granulocyte function was affected significantly by magnetic labelling with Resovist ${ }^{\circledR}$. After i.v. application into NOD/SCID mice we found that $5 \%$ of circulating granulocytes were of human origin. In addition, we were able to re-isolate these cells from mouse blood $\left(2.1 \times 10^{5}\right.$ cells $)$ enabling us to perform subsequent investigations in regards of the impact of circulation-exposure on cells.

Conclusion: Magnetic nanoparticle labelling does not impair function of platelets and granulocytes and allows MRI imaging of labelled transfused human blood cells in vivo.

Fig. 1
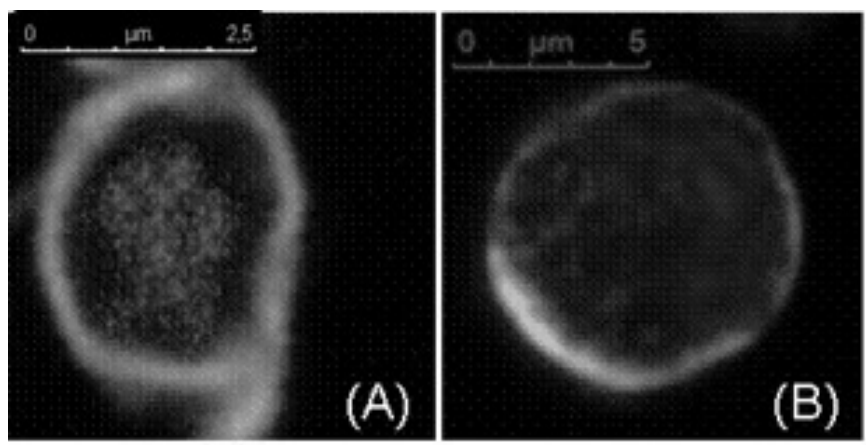
HQK-V02

Storage-induced changes of the cytosolic red blood cell proteome - an 2D DIGE/high-resolution LC-MS analysis

\author{
K. Walpurgis' ${ }^{1}$ M. Kohler ${ }^{1}$, A. Thomas ${ }^{1}$, H. Geyer ${ }^{1}$, M. Thevis ${ }^{1},{ }^{*} F$. \\ Wenzel $^{2}$ \\ ${ }^{1}$ German Sport University, Institute of Biochemistry, Cologne, Germany \\ ${ }^{2}$ Universitätsklinik Düsseldorf, ITZ, DÜsseldorf, Germany
}

Background: The storage of packed red blood cells (RBCs) is associated with the development of morphological and biochemical changes leading to a reduced posttransfusion functionality and viability of the cells. Within this study, two-dimensional polyacrylamide gel electrophoresis analysis (2D DIGE) and nano-liquid chromatography high-resolution/accuracy mass spectrometry (LC-MS) were used to analyze the storage-induced changes of the cytosolic RBC proteome and identify characteristic protein patterns and potential marker proteins for the assessment of RBC storage lesions.

Material and Methods: Leukodepleted RBC concentrates of healthy volunteers $(\mathrm{n}=12$, six female and six male) were stored according to standard blood bank conditions for 0, 7, 14, 28 and 42 days. After hemoglobin removal the RBC proteins were labeled by DIGE technique and separated using SDS-PAGE gel electrophoresis. The identification of the proteins were performed by in-gel tryptic digestion and LC-MS.

Results: A total of 189 different RBC proteins were identified comprising metabolic enzymes, chaperones and components of the cytoskeleton as well as proteins of the cellular defense or the ubiquitin-proteasome-system. Following statistical evaluation, a total of 14 protein spots were found to be significantly altered after 42 days of ex vivo storage. Especially, three proteins could be selected to be potentially useful as biomarkers for RBC aging comprising transglutaminase 2, beta actin and copper chaperone for superoxide dismutase (additionally validated by western blot analysis).

Conclusions: The observed alterations of RBC proteins during storage were only modest, but it was possible to identify three potential marker proteins for the assessment of RBC storage lesions serving as a basis for the development of a respective screening assay.

HQK-V03 ADAM-rWBC: A new tool for measurement of leucocytes
in blood components

*J. Strobel, U. Antos, R. Eckstein, J. Zingsem

Universitätsklinikum Erlangen, Transfusionsmedizin, Erlangen, Germany

Background: Cellular blood components in Germany have to be leucocyte depleted (less than $10^{\wedge} 6$ leucocytes per component, RiLi BAEK). It is therefore necessary for transfusion services to measure residual leucocytes in components after depletion. The ADAM-rWBC device (NanoEnTek, Seoul, Korea) counts leucocytes after fluorescent staining a blood sample with propidium iodide, which only dyes cells containing DNA. Staining occurs both in viable and dead cells. Then, pictures are made with a CCD camera of 200 slots on a plastic carrier, analyzed and cells counted automatically.

Methods: We measured residual leucocytes in 40 units of leucocyte depleted apheresis platelet concentrates (APLT) with three different methods: Counting the cells in the Nageotte chamber manually, by flow cytometry (LeucoCount, BD, Heidelberg, Germany) and with the ADAM-rWBC device. Furthermore we performed geometrical dilution series with six APLT samples spiked with cells from 128 leucocytes per $\mu 1$ down to 3 leucocytes per $\mathrm{ml}$ (geometrical dilution) to test for precision of the three methods.

Results: Cell counts per $\mu 1$ from the 40 units done with all three methods are shown in table 1 . All cell counts for residual leucocytes were below $10^{\wedge} 6$ cells per component, although mean cell counts were approximately 5 and 6 times higher in flow cytometry and ADAM-rWBC, respectively. The dilution series showed acceptable accuracy, especially in the range 
around the cut off for regarding a concentrate as contaminated with leucocytes. No unit with less than $10^{\wedge} 6$ leucocytes was regarded as contaminated, and on the other hand no sample spiked with more than $10^{\wedge} 6$ leucocytes was counted as having less.

Conclusion: All methods are suitable for measuring residual leucocytes in APLT. Flow cytometry is expensive and time consuming, and highly trained technicians are necessary. Counting in the Nageotte chamber is cost efficient and easy to handle, but less sensitive than the techniques compared to and very tiring for technicians. The ADAM-rWBC method is easy to perform and the results are very constant. However, due to the necessarity of loading the probe slides manually, the technician has no walk away time, as counting only takes around 90 seconds per slide. Automating the loading step of the machine could enhance the advantages of the method.

Table 1: Mean cell count $/ \mu \mathrm{l}$, minimum and maximum cell count, $\mathrm{p}$ values for group comparison

\begin{tabular}{|l|l|l|l|l|}
\hline Method & $\begin{array}{l}\text { Mean } \pm \text { standard } \\
\text { deviation }\end{array}$ & $\begin{array}{l}\text { Minimum - } \\
\text { maximum cell } \\
\text { count }\end{array}$ & $\begin{array}{l}\text { P value compared } \\
\text { to Nageotte }\end{array}$ & $\begin{array}{l}\text { P value of ADAM- } \\
\text { rWBC compared } \\
\text { to Flow cytometry }\end{array}$ \\
\hline Nageotte & $0,0725 \pm 0,088$ & $0,0-0,3$ & - & - \\
\hline Flow cytometry & $0,410 \pm 0,579$ & $0,0-2,81$ & 0,0000318 & - \\
\hline ADAM-rWBC & $0,468 \pm 0,417$ & $0,0-2,16$ & 0,0000002 & 0,031 \\
\hline
\end{tabular}

\section{HQK-V04}

\section{Influence of different preparation methods and storage solutions on platelet activation in vitro}

\author{
*W. Madla', G. Walther-Wenke ${ }^{2}$, R. Deitenbeck ${ }^{3}$, A. Grolle ${ }^{3}$, \\ M. Brodde 4 , B. Kehrel ${ }^{4}$, J. Bux ${ }^{3}$ \\ ${ }^{1}$ DRK Blutspendedienst West, Zentrale Herstellungsleitung, Hagen, \\ Germany \\ ${ }^{2}$ DRK Blutspendedienst West, Münster, Germany \\ ${ }^{3}$ DRK Blutspendedienst West, Hagen, Germany \\ ${ }^{4}$ OxProtect, Münster, Germany
}

Background: A number of methods can be used to obtain leucocyte-depleted platelet concentrates (PC) from pooled buffy coats. Apart from the manual preparation method (M-PC), it is the automated processes (OrbiSac (O-PC), TACSI (T-PC)) above all that promise a high platelet (PLT) yield as well as quality standardization. As a storage medium, plasma is frequently replaced by additive solutions (AS). The purpose of this study was to examine the effect of various preparation methods and different storage solutions (plasma, SSP, SSP+) on platelet activation in vitro.

Methods: Six PCs per preparation method (pooling of 4 buffy coats for M-PC, O-PC, T-PC) and storage solution were analyzed by flow cytometry after $0 \mathrm{~h}, 24 \mathrm{~h}$ and $72 \mathrm{~h}$ in order to assess the following in vitro parameters: Baseline fibrinogen binding, expression of P-selectin (CD62P) and granulophysin (CD63), microaggregate and microparticle formation, baseline binding of two anti-PAR-1 antibodies (providing insight into the cleavage and internalization of thrombin receptor PAR-1). The results of a total of 9 different PC preparations were compared with each other and with the results of fresh platelets obtained from whole blood.

Results: The different PCs did not reveal any significant differences in baseline fibrinogen binding to the GP IIb-IIIa receptor. In comparison with controls, the PLT from all preparations displayed increased expression of CD62P, decreasing in the sequence $\mathrm{SSP} \rightarrow \mathrm{SSP}+\rightarrow$ plasma and OrbiSac $\rightarrow$ TACSI as well as increasing in relation to time. The expression of CD63 was comparable to that of CD62P. Neither between the types of preparation nor in comparison with controls was there any evidence of differences in microaggregate formation. An increase in microparticle formation was observed after $72 \mathrm{~h}$, with plasma revealing the lowest increase. There were no significant differences between the AS, with the manual method generating the lowest amount. No differences arose between O-PC and T-PC. There were no significant variations in anti-PAR-1 antibody binding between the preparations.

Conclusion: Any differences that were found indicated that plasma offers an advantage over additive solutions, and $\mathrm{SSP}+$ an advantage over SSP (CD62P, CD63, microparticles). The effect of a longer storage period was most noticeable in an increase of microparticles and partly in CD62P. The other activation indicators remained stable, particularly the microaggregates and the PAR-1 receptor. Comparison of the preparation methods revealed the slightest differences, with minor graduations in the sequence of M-PC, T-PC, O-PC.

\section{HQK-V05}

\section{Evaluation of a proficiency panel for an external quality assurance programme for bacterial detection in platelet concentrates}

\section{${ }^{*}$ T. Vollmer ${ }^{1}$, V. Schottstedt ${ }^{2}$, L. Pichl ${ }^{2}$, K. Hourfar ${ }^{3}$, W. Sireis ${ }^{3}$ M. Schmidt ${ }^{4}$, J. Dreier ${ }^{1}$}

${ }^{1}$ Herz- und Diabeteszentrum NRW, Insitut für Laboratoriums- und Transfusionsmedizin, Bad Oeynhausen, Germany

${ }^{2}$ German Red Cross Blood Transfusion Service West, Central Laboratory, Hagen, Germany

${ }^{3}$ German Red Cross, Institute of Transfusion Medicine and Immunohematology, Frankfurt, Germany

${ }^{4}$ Reference Institute for Bioanalytics, Bonn, Germany

Background: Contamination of platelet concentrates (PCs) is the main focus of transfusion-related bacterial infections due to PC storage temperature of $22^{\circ} \mathrm{C}$, providing optimal bacterial growth conditions. In Germany, the maximum shelf-life for platelets was reduced from 5 days to 4 days to increase blood safety. Alternatively, rapid screening methods for bacterial detection in platelets were now available for routine screening to extend platelet shelf-life again to 5 days. However, the validation of these rapid bacterial detection methods represents a complicated process. Methods: The aim of this study is the development of a collaborative trial for the detection of bacteria in PCs with an external quality control panel for the application of current rapid screening methods such as Bactiflow, bacteria specific NAT, Pan Genera Detection (PGD) and BacTx. PCs were spiked with $0.03 \mathrm{CFU} / \mathrm{m}$ of different WHO bacterial standards (PEI standards) and incubated at room-temperature for 72 hours. Subsequently, bacterial titers were adjusted in platelet fluids at two concentrations $(10 \mathrm{E}+03 \mathrm{CFU} / \mathrm{ml}$ and $10 \mathrm{E}+05 \mathrm{CFU} / \mathrm{ml})$. An antibiotic concentration of 5 $\mathrm{ng} / \mathrm{ml}$ trimethoprim/sulfomethoxazol is added to each sample to prevent bacterial growth before testing. All samples were shipped to participants under temperature controlled conditions. Time schedule for testing will be limited at 6 hours, data were implemented into a web based platform. Results: Participants will pass the proficiency panel if negative controls had negative results and positive samples had positive results; samples spiked with bacteria in the range of $10 \mathrm{E}+05 \mathrm{CFU} / \mathrm{ml}$ must have a positive screening result, samples with low concentrations should have positive results, depending on the rapid detection method used.

Preliminary testing under conditions of the collaborative study using a quality control panel of samples spiked with $10 \mathrm{E}+03 \mathrm{CFU} / \mathrm{ml}$ and $10 \mathrm{E}+05$ $\mathrm{CFU} / \mathrm{ml}$ S. aureus, S. epidermidis, E. coli, K. pneumonia, S. pyogenes and $B$. cereus showed a good performance using the two rapid methods Bactiflow and bacteria specific NAT. The supplementation of antibiotics is sufficient to prevent bacterial growth in PCs but did not influence bacterial detection and still allows detection of bacteria using culture methods. Conclusion: The planned proficiency panel enables the verification of the analytical sensitivity of rapid bacterial detection systems under controlled routine conditions and represents an important contribution for blood safety. Bacterial contamination of blood components is a rare event and successful participation in proficiency testing demonstrates the efficiency of all processes of rapid bacterial screening methods and is the basis for platelet shelf-life extension. The first pilot collaborative testing with currently three participants will be performed in June this year. 


\section{HQK-V06}

Platelet units re-suspended in platelet additive solution compared with platelet units re-suspended in plasma: Less adverse events, reduced number of washing procedures and comparable rate of units per patient for ABO matched and mismatched units

\section{*W. Nussbaumer, H. Schennach, P. Mayersbach \\ ${ }^{1}$ Univ.-Klinik Innsbruck, Transfusionsmeedizin, Innsbruck, Austria}

Background: Platelet additive solution (PAS) allows a reduction of at least $65 \%$ of plasma content in platelet concentrates. Consequently human proteins in general and $\mathrm{ABO}$-antibodies in particular are significantly reduced. Platelet transfusions across the $\mathrm{ABO}$ barrier without additional manipulation (washing, reduction of plasma content by centrifugation) of the unit should become safe and effective and the frequency of adverse effects (AEs) should be reduced.

Methods: In our blood bank we introduced PAS in a 65:35 ratio (PAS vs. plasma) for all our platelet units beginning of 2009. PAS-3M (SSP+, Macopharma, Langen, BRD) is used for apheresis as well as for pooled platelets. Using our computerized hemovigilance system, which requests reports from all transfusions and not only AEs, we analyzed platelet transfusion reports from 2009 to 2013 (PAS) for report efficacy, rate of AEs, and rate of washing procedures. Data are given as a total as well as spitted for ABO matched and mismatched transfusions and compared with data from 2004 (plasma), a year all units were re-suspended in 100\% plasma. Results: A total of 19450 requested transfusions were registered for the PAS group and 5141 for the plasma group. Report efficacy was $93.78 \%$ vs. $94.16 \%$ (PAS group vs. plasma group, respectively). Rate of ABO matched transfusions remains similar between both groups $(72.12 \%$ vs. $73.54 \%$, PAS vs. plasma). Washing procedures were reduced from $17.2 \%$ to $1.19 \%$, mainly responsible units containing ABO antibodies directed against recipients $\mathrm{ABO}$ antigens $(97.84 \%$ vs. $2.27 \%$, plasma vs. PAS) Rate of AEs was decreased from $0.76 \%$ to $0.42 \%$ in general, from $0.77 \%$ to $0.45 \%$ for matched units and from $0,79 \%$ to $0.34 \%$ for $\mathrm{ABO}$ mismatched units (plasma vs. PAS). Number of platelet units transfused per patient was 6.09 vs. 4.34 (plasma vs. PAS).

Conclusion: We found a significant decrease $(p<0.01)$ of AEs in the PAS group. Decrease was highly significant for the matched units $(\mathrm{p}<0.01)$ and slightly significant for the mismatched units $(p=0.03)$. Also washing procedures could be minimized to $1.19 \%$. Regarding the transfusion efficacy, our data lack information about CCI or increment. However, the lower number of units per patient for the PAS group may also be an indirect measurement that PAS units are at least not inferior to plasma units. Controlled, prospective studies may confirm our retrospective findings in the future.

\section{Poster: Herstellung \& Qualitätskontrolle}

\section{HQK-P01}

\section{Production of Autologous Serum Eye Drops (ASE) under the terms of German legal requirements}

\section{${ }^{*}$ R. Conradi, S. Runkel, W.E. Hitzler, D. Marandiuc ${ }^{1}$ \\ Universitätsmedizin Mainz, Transfusionszentrale, Mainz, Germany}

Background: Autologous serum manufactured for application as eye drops underlies the strict legal specifications of the German law on drugs (AMG). The production has to take place under controlled conditions, based on the guidelines for good manufacturing practice (EU-GMP). Additionally the Pharmacopoea Europaea (Ph. Eur.) is the legal basis for preparations to be administered to the eye. We describe our experience in ASE production related to the legal requirements, autologous blood donation and serum processing.
Methods: Our requirements were:

1. The production in a closed system to avoid the expensive and time consuming clean-room facilities.

2. The standardized blood collection and processing to yield reproducible quality.

3. A quick and effortless procedure for patients with flexible amount of phials and easy application of eye drops.

4. The compliance with German legal requirements.

For autologous blood donation we used a TF304.1 blood bag. After clotting the serum was transferred into a chained phial system TF30 (Meise Medizintechnik GmbH, Schalksmühle). This system is closed and has 30 phials of $1.5 \mathrm{~mL}$ each.

The specifications for residual cell counts were: RBC

Results: The following aspects could be implemented or validated:

1. For standardization of whole blood collection we examined the volume of blood which had to be drawn to fill 1, 2, or 4 chains of phials with serum. We came to a volume of 140, 260 and $460 \mathrm{ml}$ of whole blood.

2. A decision about the blood volume to donate was needed for every patient with respect to illness, age and condition of the patient.

3. The Bactec FX (Becton Dickinson) microbiological testing system was validated for serum samples (Ph. Eur.).

4. Logistics and validation for shipment of frozen ASE on dry ice and involving a public pharmacy under the terms of German law.

5. Adjustment of the clotting conditions of whole blood from patients with delayed drug-dependent coagulation (heparin, vitamin $\mathrm{K}$ antagonists).

Conclusion: We established and described a process for standardized manufacture of ASE under legal conditions. This process is suitable for nearly every patient.

ASE produced in a closed system can be stored at $-20{ }^{\circ} \mathrm{C}$ for 112 days. An autologous blood donation of $460 \mathrm{~mL}$ can assure ASE for a period of almost 4 months. Therefore patients with chronic disease and in good condition must donate blood only 3-4 times a year. Until now for all our patients (age 8 to 90 years) it was possible to produce ASE in a satisfying matter. The assumption of costs has to be clarified in every single case by the patient.

\section{HQK-P02}

\section{A stress test for stored platelets}

${ }^{\star}$ B. Baumann-Baretti ${ }^{1}$, B. Brys ${ }^{1}$, G. Jaster ${ }^{1}$, K. - P. Krause ${ }^{1}$, M. Heiden ${ }^{2}$, W. Schwarz ${ }^{2}$

${ }^{1}$ Haema AG, Qualitätskontrolle, Berlin, Germany

${ }^{2}$ Paul-Ehrlich-Institut, Hämatologie/Transfusionsmedizin, Langen, Germany

Background: Storage at $4{ }^{\circ} \mathrm{C}$ largely alters in vitro function of platelets. However, preliminary investigations demonstrated that platelets stored in plasma are able to restore hypotonic shock resistance (HSR) and are fully activatable upon stimulation with TRAP-6 when temporarily stored at 4 ${ }^{\circ} \mathrm{C}$. It was of interest if platelet concentrates (PC) stored in additive solution as well as additionally for pathogen inactivation treated $\mathrm{PC}$ would be able to maintain platelet function in a comparable manner.

Methods: The Trima 6.0 apheresis device (Terumo BCT) was used to collect PC suspended in SSP+ additive solution (MacoPharma) $(n=8)$ or plasma $(n=3)$. Pathogen inactivation was performed with the Intercept $^{\mathrm{TM}}$ Blood System for Platelets (Cerus Europe BV). Except during Amotosalen/UVA treatment in pathogen inactivation, all PC continually rotated on flatbed agitators. Standard storage temperature $\left(22 \pm 2{ }^{\circ} \mathrm{C}\right)$ of $\mathrm{PC}$ in plasma (A) was changed to $4^{\circ} \mathrm{C}$ on day (d) 1 to $\mathrm{d} 3$ for 72 hours. $\mathrm{PC}$ in SSP+ were collected as Double PC (D-PC); one of the D-PC, (B), was stored at $22 \pm 2^{\circ} \mathrm{C}$ until pathogen inactivation of the corresponding D- PC, (C), was performed. Then the storage temperature for (B) and (C) was changed to $4^{\circ} \mathrm{C}$ for 72 hours lasting from d2 to d4. For all PC, quality control was conducted daily from d1 to d5 and on d7. Additionally CD62P expression quantified on FACS (Calibur, Becton Dickinson), blood gas analysis, lactate and glucose were tested. 
Results: When stored at $4^{\circ} \mathrm{C}$ the $\mathrm{pH}$ in the PC slightly increased (A) or rather stayed steady (B and C). Turning back to $22 \pm 2{ }^{\circ} \mathrm{C}, \mathrm{pH}$ decreased with a most pronounced drop in (C), staying on 6.4 until d7 (table 1).

During storage at $4^{\circ} \mathrm{C}$, platelets of all PC showed less (A) and (B) or no (C) further CD62P expression upon TRAP-6 stimulation: The continuous functional loss stopped for (A) and (B) after relocation to $22 \pm 2{ }^{\circ} \mathrm{C}$ and yet recovered in (B). Platelets of (C) became completely insensitive towards TRAP- 6 within the first day at $4{ }^{\circ} \mathrm{C}$ remaining irreversible even when relocated to $22 \pm 2{ }^{\circ} \mathrm{C}$ (table2).

Due to storage at $4^{\circ} \mathrm{C}$ HSR decreased in platelets of all PC: For (A) and (B) the decrease averaged $20 \%$ while in (C) it hit $60 \%$. Relocated to $22 \pm 2{ }^{\circ} \mathrm{C}$, platelets of (A) and (B) showed increased HSR while those of (C) rapidly lost HSR being hardly detectable at $\mathrm{d} 5$ and no more at $\mathrm{d} 7$. Conclusion: Platelets suspended in plasma or SSP+ demonstrated comparable in vitro functionality during storage of $4^{\circ} \mathrm{C}$ and after relocation to $22^{\circ} \mathrm{C}$. In contrast, platelets treated for pathogen inactivation completely lost their HSR, showed high preactivation and could not be further activated with TRAP-6. In addition $\mathrm{pH}$ became acidic. It is assumed that platelets from pathogen inactivated PC cannot repair the cold storage induced loss of membrane integrity due to missing protein neosynthesis.

Table 1: $\mathrm{pH}$ during storage

\begin{tabular}{|c|c|c|c|c|c|c|c|}
\hline & $\mathrm{d} 0$ & $\mathrm{~d} 1$ & $\mathrm{~d} 2$ & $\mathrm{~d} 3$ & $\mathrm{~d} 4$ & $\mathrm{~d} 5$ & $\mathrm{~d} 7$ \\
\hline \multirow{2}{*}{ A } & $6,95 \pm$ & $7,04 \pm$ & $7,10 \pm$ & $7,12 \pm$ & $7,00 \pm$ & $6,94 \pm$ & $6,78 \pm$ \\
& 0,02 & 0,02 & 0,03 & 0,03 & 0,08 & 0,09 & 0,11 \\
\hline \multirow{2}{*}{ B } & & $6,98 \pm$ & $7,00 \pm$ & $6,96 \pm$ & $6,95 \pm$ & $6,86 \pm$ & $6,85 \pm$ \\
& & 0,03 & 0,03 & 0,02 & 0,02 & 0,06 & 0,04 \\
\hline \multirow{2}{*}{ C } & & $6,75 \pm$ & $6,76 \pm$ & $6,72 \pm$ & $6,69 \pm$ & $6,42 \pm$ & $6,43 \pm$ \\
& & 0,06 & 0,06 & 0,06 & 0,08 & 0,05 & 0,10 \\
\hline
\end{tabular}

Table 2: Increase of CD 62 expression after stimulation with TRAP in [\%]

\begin{tabular}{|c|c|c|c|c|c|c|c|}
\hline & d0 & d1 & d2 & d3 & d4 & d5 & d7 \\
\hline \multirow{2}{*}{ A } & $81,5 \pm$ & $67,5 \pm$ & $65,1 \pm$ & $54,3 \pm$ & $24,0 \pm$ & $18,6 \pm$ & $24,8 \pm$ \\
& 8,8 & 2,8 & 11,6 & 4,0 & 1,9 & 8,9 & 9,0 \\
\hline \multirow{2}{*}{ B } & & $79,0 \pm$ & $40,1 \pm$ & $23,4 \pm$ & $15,4 \pm$ & $33,7 \pm$ & $31,5 \pm$ \\
& & 8,9 & 9,9 & 12,9 & 6,1 & 6,4 & 9,4 \\
\hline \multirow{2}{*}{ C } & & $46,3 \pm$ & $0,9 \pm$ & $-2,8 \pm$ & $-2,4 \pm$ & $0,6 \pm$ & $1,5 \pm$ \\
& & 17,5 & 5,0 & 4,9 & 2,2 & 0,5 & 3,3 \\
\hline
\end{tabular}

HQK-P03

In-vitro quality of Red Blood Cells, pooled Platelet Concentrates, and Plasma Products prepared from overnight stored whole blood by automated blood component processing machine TACSI

\section{${ }^{*}$ G. Matthes, R. Beltzig}

Universitätsklinikum Leipzig AöR, Institut für Transfusionsmedizin, Leipzig, Germany

Background: The new TACSI WB protocol processes 6 whole blood (WB) units into 6 plasma, 6 red blood cell (RBC), and 6 buffy coat (BC) units. TheTACSI PLprotocol processes 4-6 BC into a pooled platelet concentrate (PC). The study aims to collect data for automatic blood component processing and for in-vitro quality analysis of TACSI produced $\mathrm{RBC}, \mathrm{PC}$, and plasma products prepared from overnight stored WB.

Methods: $450 \mathrm{~mL}$ of WB donation from 78 blood donors was collected into a TACSI WB kit. These were processed from overnight stored WB within the TACSI machine into three components: RBC, PC, Plasma $\mathrm{RBCs}$ were leucodepleted post processing through the integrated Imuflex $\mathrm{RBC}$ filter. PCs were processed from $4 \mathrm{BCs}$ and 1 plasma unit and leucodepleted through the integrated Imuflex PLT filter during processing. No filtration is needed for plasma since they are leucodepleted already through centrifugation. Parameters for in-vitro quality of RBC, PC, and plasma units were selected and measured according to the requirements of guidelines (RBC at day 1, 7, 14, 21, 28, 35, 42, 45; PC at day 1, 3, 4, 5,7 ; plasma at day 1,35 ).

Results: The results of this study prove that the TACSI WB System automates the manual steps of blood component processing. Table 1 represents data for the three final products at day 1 after collection. All RBC, PC, and plasma units show from beginning up to the end of storage good quality parameters. Table $2 \& 3$ summarize the monitoring for storage quality. The recovery for all processed products amounted to $72 \%$ for RBCs, to $75 \%$ for PCs, and to $80 \%$ for plasma.

Conclusion: Automation with TACSI WB technology provides consistent leucodepleted RBCs, PCs with a high recovery created by four pooled $\mathrm{BCs}$, and very pure plasma products. Compared to conventional methods manual steps can be saved significantly. All TACSI WB processed blood products are comparable in storage data with known good quality by meeting European and national standards.

\begin{tabular}{|c|c|c|c|c|c|c|c|}
\hline $\mathrm{RBC}$ & $\begin{array}{c}\text { Vol. } \\
m L\end{array}$ & $\begin{array}{l}\text { rWBC } \\
W B C / \text { unit }\end{array}$ & $\begin{array}{c}\mathrm{pH} \\
22^{\circ} \mathrm{C}\end{array}$ & $\begin{array}{l}\mathrm{Hb} \\
g / \text { unit }\end{array}$ & $\begin{array}{l}\text { PLT } \\
\text { Gptunit }\end{array}$ & $\begin{array}{c}\mathrm{K}+ \\
\text { mmolunit }\end{array}$ & $\begin{array}{c}\text { Free } \mathrm{Hb} \\
m g / g h b\end{array}$ \\
\hline Number & 20 & 20 & 20 & 20 & 20 & 20 & 20 \\
\hline Average & 254 & $5,43 E+05$ & 7,13 & 48 & 2 & 1,90 & 9,3 \\
\hline S.D. & 14 & $8,50 \mathrm{E}+05$ & 0,06 & 5 & 1 & 0,16 & 4,0 \\
\hline PC & Vol. & rwBC & $\mathrm{pH}$ & PLT & MPV & \multicolumn{2}{|c|}{ Aggregation } \\
\hline & $m L$ & WBC/unit & $22^{\circ} \mathrm{C}$ & *10e11/unit & fil & Coll $(\%)$ & $A D P(\%)$ \\
\hline Number & 17 & 17 & 17 & 17 & 17 & 17 & 17 \\
\hline Average & 298 & $8,98 \mathrm{E}+04$ & 7,27 & 3,15 & 6,2 & 77 & 60 \\
\hline S.D. & 9 & $4,02 \mathrm{E}+04$ & 0,12 & 0,45 & 0,4 & 6 & 14 \\
\hline Plasma & Vol. & rWBC & $\mathrm{pH}$ & F V & F VIII & F IX & TP \\
\hline & $m L$ & WBC/unit & $22^{\circ} \mathrm{C}$ & $\%$ & $\%$ & $\%$ & g/unit \\
\hline Number & 16 & 16 & 16 & 16 & 16 & 16 & 16 \\
\hline Average & 257 & $1,34 E+05$ & 7,24 & 99 & 105 & 114 & 16 \\
\hline S.D. & 17 & $1,47 \mathrm{E}+05$ & 0,08 & 21 & 34 & 21 &  \\
\hline
\end{tabular}

Table 2: Quality and storage monitoring of RBC products.

\begin{tabular}{|c|r|rrr|r|}
\hline \multicolumn{6}{|c|}{ Table 2: Quality and storage monitoring of RBC products. } \\
\hline RBC & pH & ATP & Hbe & K+ & $\begin{array}{c}\text { Hemolysis } \\
\text { Rate }\end{array}$ \\
\hline & $22^{\circ} \mathrm{C}$ & $\mu$ molig Hb & mg/dL & mmoll & $\%$ \\
\hline Number & 20 & 20 & 20 & 20 & 20 \\
day1: Average & 7,13 & 5,09 & 17 & 1,90 & 0,03 \\
S.D. & 0,06 & 0,35 & 8 & 0,16 & 0,01 \\
\hline day7: Average & 6,94 & 5,30 & 30 & 17,44 & 0,06 \\
\hline S.D. & 6,95 & 5,28 & 37 & 17,42 & 0,07 \\
day21: Average & 6,72 & 4,14 & 68 & 36,92 & 0,12 \\
\hline S.D. & 0,06 & 0,37 & 35 & 2,88 & 0,06 \\
\hline day35: Average & 6,65 & 2,82 & 120 & 49,19 & 0,21 \\
S.D. & 0,04 & 0,42 & 69 & 3,62 & 0,12 \\
\hline day42: Average & 6,58 & 2,30 & 178 & 53,68 & 0,31 \\
\hline S.D. & 0,04 & 0,36 & 97 & 4,14 & 0,18 \\
\hline
\end{tabular}

\begin{tabular}{|c|c|c|c|c|}
\hline $\mathrm{PC}$ & $\mathrm{pH}$ & $\begin{array}{l}\text { Aggregation } \\
\text { ADP }\end{array}$ & $\begin{array}{l}\text { Aggregation } \\
\text { Collagen }\end{array}$ & MPV \\
\hline & $22^{\circ} \mathrm{C}$ & $\%$ & $\%$ & $\mathrm{fL}$ \\
\hline Number & 17 & 17 & 17 & 17 \\
\hline day1: Average & 7,27 & 77 & 60 & 6,2 \\
\hline S.D. & 0,12 & 6 & 14 & 0,4 \\
\hline day 3 : Average & 7,59 & 69 & 39 & 5,6 \\
\hline S.D. & 0,10 & 10 & 14 & 0,4 \\
\hline day4: Average & 7,50 & 73 & 30 & 5,5 \\
\hline S.D. & 0,07 & 5 & 7 & 0,3 \\
\hline day5: Average & 7,47 & 64 & 34 & 5,4 \\
\hline S.D. & 0,06 & 6 & 7 & 0,4 \\
\hline day7: Average & 7,41 & 57 & 21 & 5,3 \\
\hline S.D. & 0.10 & 16 & 10 & 0.4 \\
\hline
\end{tabular}




\section{HQK-P04}

\section{In vitro Evaluation of Buffy Coat derived Platelet concentrates resuspended in $\mathbf{8 0} \%$ or $\mathbf{8 5} \%$ PAS IV}

\author{
*U. Gravemann', T. Volgmann" , A. Uyttenhove ${ }^{3}$, R. Philipp 4 , \\ B. Lambrecht ${ }^{1}$, J.-M. Payrat ${ }^{3}$, A. Seltsam ${ }^{1}$ \\ 'DRK-Blutspendedienst NSTOB, Forschung und Entwicklung, Springe, \\ Germany \\ ${ }^{2}$ DRK-Blutspendedienst NSTOB, Springe, Germany \\ ${ }^{3}$ Fenwal Europe, Mont-Saint-Guibert, Germany \\ ${ }^{4}$ Fenwal Germany, München, Germany
}

Background: PAS IV (InterSol-G ${ }^{\mathrm{TM}}$; Fenwal) is a new additive solution containing glucose that has been developed for storage of platelet concentrates (PCs) with residual plasma of less than $30 \%$. In vitro quality parameters of buffy coat (BC)-derived PCs stored in PAS IV with a plasma ratio of $20 \%$ or $15 \%$ were evaluated over a storage period of 7 days.

Methods: Selection of volunteer donors was based on local standard practices. Whole blood units were processed after overnight storage at room temperature. High speed centrifugation was applied and BCs were separated. In a pool and split design, $8 \mathrm{ABO}$ compatible BCs were pooled and split into two pools that were supplemented by PAS IV or by PAS IV with a small amount of additional plasma to reach a final plasma ratio of $15 \%$ and $20 \%$, respectively. Units $(n=12$ per study arm) were stored for up to 7 days and were regularly sampled for in vitro assays.

Results: The PC volumes were $283 \pm 18 \mathrm{~mL}$ (PAS IV-20\%) and $282 \pm$ $14 \mathrm{~mL}$ (PAS IV-15\%). The PLT concentration was similar in both PC types (PAS IV-20\%: $10.2 \pm 0.7 \times 10^{8} / \mathrm{mL}$; PAS IV-15\%: $10.1 \pm 0.6 \times 10^{8}$ $\mathrm{mL}$ ). The $\mathrm{pH}$ at $22^{\circ} \mathrm{C}$ measured at the end of storage was above 6.4 for both products. The mean glucose concentration was above $11.0 \mathrm{mmol} / \mathrm{L}$ at the end of storage independent of the plasma content. Lactate reached concentrations of $8.0 \pm 0.7 \mathrm{mmol} / \mathrm{L}$ in PAS IV-20\% PCs and of $7.5 \pm 0.6$ $\mathrm{mmol} / \mathrm{L}$ in PAS IV-15\% PCs. The aggregation response after collagen stimulation $(10 \mu \mathrm{g} / \mathrm{mL})$ was similar for both types of PCs (PAS IV-20\%: $58 \pm 13 \%$; PAS IV-15\%: $56 \pm 18 \%$, day 7). CD62 expression was significantly higher in PAS IV-20\% PCs than in PAS IV- $15 \%$ PCs from day 5 ( $30 \pm 2 \%$ vs. $26 \pm 2 \%$; $\leq 0.001$, day 7 ). PAC- 1 antibody binding values were similar for both PC types throughout the 7 days (PAS IV-20\%: $66 \pm$ 5\%; PAS IV-15\%: $65 \pm 4 \%$, day 7).

Conclusion: PLT quality is well maintained in PAS IV during the 7 days storage period with both plasma ratios. This study shows that it is feasible to reduce the plasma ratio in $\mathrm{BC}$-derived PCs down to $15 \%$. PCs with minimal plasma content may help to reduce the occurrence of transfusion reactions such as transfusion related acute lung injury (TRALI) or allergic reactions for which plasma proteins have been implicated as the causative factor.

\section{HQK-P05}

\section{Efficacy and safety of fresh frozen plasma as precondition for successful Plasma Exchange}

\section{${ }^{*}$ C. Weinigel, C. Weise, S. Rummler, D. Barz}

Universitätsklinikum Jena, Institut für Transfusionsmedizin, Jena, Germany

Background: We developed a leukocyte and platelet-depleted pathogen reduced single donor apheresis plasma via filtration with Plasmaflex PLAS4 and Intercept Blood System for Plasma. This pathogen inactivated plasma (PIP) was the first to receive marketing authorisation from the PEI in 2011 (PEI.H.11468.01.1). SD plasma and PIP were applied for plasma exchange therapy (TPE) without randomization depending on availability.

Methods: During the first two years of clinical application (03/2011 04/2013) altogether 1546 PIP-units were transfused during TPE. 162 TPE procedures were performed on 45 patients. 793 units of SD Plasma were transfused in 33 patients within $76 \mathrm{TPE}$. The main indications were transplant rejection episodes, chronic inflammatory neurological diseases and thrombocytopenia. Data were assessed retrospectively.
Results: In total TPE was tolerated well, no thrombotic or bleeding episodes were seen. Only 11 mild transfusion reactions were documented in the PIP group (6.8\%) vs. 10 reactions in the SD group (13.2\%). Coagulation parameters (Quick, aPTT, Fibrinogen) were analysed before and after TPE. The Quick value decreased by $15 \%$ when using PIP vs. $13 \%$ with SD, aPTT increased $2 \%$ with PIP vs. a $7.6 \%$ decrease under SD and fibrinogen decreased $31 \%$ (PIP) vs. $26 \%$ (SD).

Conclusion: The pathogen reduced plasma has shown a good tolerability in routine clinical use for plasma exchange therapy. No unexpected transfusion reactions were observed. PIP has proven to be at least comparable to SD plasma. Possibly, further monitoring may show PIP being superior to SD plasma.

\section{HQK-P06}

\section{Automated technology for transporting, scanning and scaling of plasma products from whole blood donations}

\section{U. Schäfer', *P. Schauwecker', K. Janetzko², H. Schrezenmeier',} M. Wiesneth ${ }^{1}$

${ }^{1}$ Institute UIm, German Red Cross Blood Transfusion Service BadenWürttemberg - Hessen, Germany, Ulm, Germany

2Institute Mannheim, German Red Cross Blood Transfusion Service Baden-Württemberg - Hessen, Germany, Mannheim, Germany

Background: About 850.000 fresh frozen plasma (FFP) from whole blood donations are produced yearly inGermany. Given that all available blood component separators cannot assess the weight of the blood components according the regulatory requirements, FFPs are currently scanned and scaled manually in order to define the exact volume for labeling each product. Here we demonstrate an automated combined transport, scanning and scaling system (Garvens METTLER TOLEDO) for manufacturing of plasma products.

Methods: The automated transport, scanning and scaling system consists of a haul track (length $12 \mathrm{~m}$, width $32 \mathrm{~cm}$ ), which can be loaded with plasma bags from each separator, a calibrated scale (precision $\pm 0.1 \mathrm{~g}$, weighing range $1 \mathrm{~g}$ to $1.000 \mathrm{~g}$ ) and two automated bar code readers with a capacity to scan 400 plasma bags/h. The conveyor belt runs with $10 \mathrm{~m} /$ min, transports the plasma bags from the separator to the freezer and the bags are scanned and scaled automatically in the meantime.

Results: During the last 3 months about 60.000 plasma products were transported, scanned and scaled automatically, thus the volume was calculated and saved electronically with the product code. Read fault errors of the product codes occurred in only $1.09 \%$ and were shown on the display with an audible alarm. For validation purposes the results from the automated system were compared to the results from manual scaling with a calibrated precision balance (Sartorius) $(\mathrm{n}=100, \Delta \mathrm{m}=-0.1 \mathrm{~g}$, range $=$ $-0.4 \mathrm{~g}$ to $+1.4 \mathrm{~g}$ ). By substituting for the scaling using the separators and the current manual procedure, the presented automated technology would save about $15.000,-€$ per year when considering an annual amount of 250.000 whole blood preparations. Quality of plasma products like factor VIII content was not influenced of the automated technology.

Conclusion: The automated transport, scanning and scaling system for plasma products is safe and in accordance with the regulatory requirements. The system delivers high quality and productivity and can also be used for other blood products like red cell concentrates.

\section{HQK-P07 \\ In vitro evaluation of platelets stored in three different additive solutions}

\section{${ }^{*}$ G. Leitner, J. List, M. Horvath, B. Eichelberger, P. Jilma}

Allgemeines Krankenhaus, Wien, Klinik für Blutgruppenserologie und Transfusionsmedizin, Wien, Austria

Background: Usually platelets are stored in $100 \%$ plasma. Plasma contains all nutritive factors to store platelets for 5 days with minimal metabolic changes. In the eighties first attempts were made to replace plasma 
by platelet additive solutions (PAS) to gain plasma for other purposes. Since pathogen inactivation (PI) is an upcoming topic in transfusion medicine additive solutions are again a matter of interest because PI methods require PAS with or without plasma as storage media.

Our aim was to examine in a prospective analysis the impact of three different PAS on quality of platelets concentrates stored for up to 7 days. Methods: All donors $(n=12)$ were recruited from our routine multicomponent donor pool ( 3 females / 9 males, median age 43 yrs; range 26-57) and fulfilled the requirements for triple platelet concentrate (PC) harvests. All procedures were performed with TrimaAcell ${ }^{\circledR}$ soft ware version 6.0 (TerumoBCT). Automatic PAS addition was switched off. The highly concentrated product was divided into three equal parts and the additive solutions $\left(\mathrm{Composol}^{\circledR}\right.$ or $\mathrm{SSP}+{ }^{\circledR}$ or Intersol ${ }^{\mathrm{TM}}$ ) were added to a final concentration of $56 \%$.

PCs were stored at $22^{\circ} \mathrm{C}\left( \pm 2{ }^{\circ} \mathrm{C}\right)$ under continuous agitation untill expiration. Samples were drawn under aseptic conditions on days 1, 5 and 7 to measure the extent of storage lesions by means of $\mathrm{pH}, \mathrm{O} 2, \mathrm{CO} 2$, Glucose, $\mathrm{LDH}$, lactate, MPV and aggregation response to thrombin receptor agonist peptide-6 (TRAP-6 $50 \mu \mathrm{m}$ ). The study was performed in a cross over design.

Results: The yield for each PC was mean $2.28 \times 10^{11}$ (SD 0.3). On day 1 there was no significant differences for any of the tested parameters. In all products minimal changes were measured for $\mathrm{pH}, \mathrm{MPV}, \mathrm{O} 2$ and $\mathrm{CO} 2$ during the 7 days of storage. Glucose decreased and LDH and lactate increased in all units over time. On day 7 glucose was significant lower in Intersol PCs than in Composol and SSP+ PCs $(12 \mathrm{mg} \%$ on day $7 \mathrm{vs}$ $38 \mathrm{mg} \%$ in Composol and $36 \mathrm{mg} \% \mathrm{SSP}+$ products). In accordance LDH and lactate were significantly higher in Intersol units day $(224 \mathrm{U} / \mathrm{L}$ and $16.6 \mathrm{mmol} / \mathrm{L})$ compared to Composol (100 U/L and $12.2 \mathrm{mmol} / \mathrm{L})$ and $\mathrm{SSP}+(143 \mathrm{U} / \mathrm{L}$ and $12.8 \mathrm{mmol} / \mathrm{L})$. Intersol PCs showed higher baseline activation during the whole storage time and reduced response to TRAP on day 7 ( $\mathrm{p}<0.05)$.

Conclusion: For 7 days of storage SSP+ and Composol are equal in maintaining glucose and keeping acid metabolites low. The clinical meaning of the low response to agonists on day 7 in Intersol platelets has to be investigated.

\section{HQK-P08}

\section{The automatization in granulocyte collection using the Optia device eases the procedure and provides concentrates of high quality}

\section{${ }^{*}$ G. Leitner, V. Kolovratova, M. Horvath, N. Worel}

Allgemeines Krankenhaus, Wien, Klinik für Blutgruppenserologie und Transfusionsmedizin, Wien, Austria

Background: The therapeutic effect of granulocyte transfusions in leukopenic patients with severe infections refractory against antibiotics or antimycotics is generally accepted. The challenge is the ability to collect sufficient numbers of granulocytes to induce eradication of pathogens. In the last decades several approaches were initiated to enable the collection of a therapeutic dose (4 $10^{10}$ granulocytes per day) of neutrophiles (ANC). The last innovation in this field is the fully automated Optia ${ }^{\circledR}$ device / Cobe (COBE BCT, Lakewood, CO).

Methods: In a prospective analysis we aimed to show that the fully automated system of Optia/Cobe provides granulocyte concentrates not inferior in terms of yield, purity and donor comfort to the current devices. 26 donors, mobilized with prednisolon $(\mathrm{P})$, were recruited from our routine multicomponent donor pool ( 2 females / 24 males, median age 42 yrs, range 27-63). These volunteers donated 1 concentrate. 14 collections were obtained from 6 donors, all relatives of the recipients, $(1$ female $/ 5$ males, median age 30 yrs, range 24-59) who were mobilized with G-CSF at a dose of $5 \mu \mathrm{g} / \mathrm{kg}$ bodyweight (BW). All collections were performed with Optia ${ }^{\circledR}$ device according to the instructions of the manufacturer. A mean of 6.1L (SD 0.3) of donor's blood volume was processed within 120 minutes. Anticoagulation was done with a mixture of Hetastarch $6 \%$ and sodium citrate $46.7 \%$.
Results: None of the donors experienced any side effects. The gain of $\mathrm{BW}$ was in mean $0.4 \mathrm{~kg}$ for the routine $(\mathrm{P})$ - and $0.2 \mathrm{~kg}$ for the G-CSF group. A mean of $449 \mathrm{~mL}$ (SD 61) was collected with a purity of in mean $82 \%$ (SD 6.1) ANC for both mobilization groups. As expected a significant difference was found in the absolute yield of ANC in the products, 5.9 (SD 3.1) $\times 10^{10}$ in the G-CSF group vs $1.9(\mathrm{SD} 0.5) \times 10^{10}$ in the $\mathrm{P}$ group. Compared with historical data the product volume as well as the absolute ANC yield in the P group was significantly higher in Optia than in collections done with Spectra ${ }^{\circledR} /$ Cobe: $449 \mathrm{~mL}$ vs $225 \mathrm{~mL}$ and $1.9 \times 10^{10}$ $(\mathrm{SD} 0.5)$ vs $1.3 \times 10^{10}(\mathrm{SD} 0.6)$ respectively, $\mathrm{p}<0.5$.

Conclusion: Granulocyte collections with the fully automated Optia ${ }^{\circledR}$ device are safe for donors. The procedure requires less man-power than Spectra $^{\circledR}$ as a continuous visual control of the collection-line is negligible accompanied by high quality of the products.

\section{HQK-P09 \\ Autologous serum eye drops (ASEDs) in the therapy of ocular surface diseases: A first one year data evaluation from the clinical and manufacturing point of view (March 2012-April 2013)}

\section{${ }^{*}$ P.-J. Viorica-Maria, M. Störmer, B. Assefa, S. Radojska, L. Oustianskaia, I. Loquai, B. Gathof \\ Uniklinik Köln , Transfusionsmedizin , Köln, Germany}

Background: The therapy with ASEDs containing epitheliothrophic factors (growth factor, fibropectin and vitamins) may lead in patients suffering especially from ocular surface disorders (i.e. dry eye, persistent epithelial defects) to a rapid and essential improvement of clinical symptoms. This study evaluated our first experience of preparation and therapy with ASEDs indicated by 30 patients.

Methods: Thirty ambulant patients treated with ASEDs have been evaluated over one year retrospectively. The data included age and history of patients, clinical diagnosis and technical problems during product's manufacturing and storage. ASEDs were manufactured in a "closed" system in $93 \%$ of patients $(28 / 30)$ using specific donation bags and ocular applicators systems (Meise GmbH, Germany). In 2 (7\%) of patients a minor volume of serum samples were collected using serum monovette tubes. Serum separation and transfer into the sampling bag of the closed ASEDs system were performed under GMP conditions in the clean zone. The ASEDs applicators need to be stored in deep freezer at $-18^{\circ} \mathrm{C}$. The delivery of occular applicators to the patients and their storage is performed $<-18^{\circ} \mathrm{C}$.

Results: We registered one blood donation per patient in $63 \%(19 / 30)$, 2 donations in $27 \%(8 / 30)$ and 5 donations in $10 \%(3 / 30)$ of all patients. Initiation of ASEDs manufacturing in our laboratories required a complex organization. At the beginning of manufacturing in a close system, technical incidents as 3 breaking blood bags during centrifugation occured. Evaluations showed that the breaks occured at a low place of the bags during the vertical centrifugation. After the bags' horizontal centrifugation this incident didn't occured anymore. A special ASEDs delivery system using shipment on dry ice through external pharmacies has been introduced and validated. A further technical incident occured during storage due to a defect of a pharmacy's deep freezer which led to loss of contained ASEDs.

Conclusion: The manufacture of ASEDs in closed and open system has been successfully validated and implemented in routine production. All repeated donating patients (7-82 years) reported an improvement of clinical symptoms reflected in a better vew and eye luminosity, substantial reduction of eye inflammatory signs and dryness. Due to benefits of ASEDs application, an increasing demand of this therapy is expected. But, the ASEDs' delivery through pharmacies is very time consuming: in this sense, a change of German regulations allowing a direct delivery to the patients is urgently necessary. 


\section{HQK-P10}

\section{From CAPA-Workflows to PQR in one Database}

${ }^{*}$ H. Clemm, S. Kießig

Ainea AG, Ludwigshafen, Germany

Background: The preparation of a Product quality review is mandatory since 2005 (ICH Q7A) Therefore a single database summarizing as much as possible data is helpful. A wide definition of CAPA (Corrective Action - Preventive Action) is a prerequisite.

Methods: Medical complaints, complaints by the recipient, audit findings, results out of specification (OOS), deviations in quality control, manufacturing validation, and microbiological controls, are often handled separately. All of them undergo a comparable process: a corrective action is required and if a distinct level is passed also a preventive action (a CAPA) is required. Therefore a common workflow can be established. Also post donation reporting and look back procedures can be treated as a CAPA process. The tracking, trending and reporting of epidemiological data should be included here.

Results: A single database not only to document the different kinds of deviations and complaints is required. The same database should fulfill also specifications of a document control system as guided documents flow, document scheduling, documented training, even under decentralized conditions.

The analysis of existing and planned processes is prerequisite for an introduction of an individualized CAPA workflow into a DCM.

Conclusion: The set up of workflows in a single database requires a lot of analytical work. The reports from the database can be set up to minimize the efforts in $\mathrm{PQR}$ reporting.

\section{HQK-P11}

\section{Platelet-derived extracellular vesicles influence the quality of platelet concentrates}

\section{*A. Black, A. Pienimäki-Römer, E. Orsó, G. Schmitz}

University Hospital Regensburg, Institute for Laboratory Medicine and Transfusion Medicine, Regensburg, Germany

Background: Platelet-derived extracellular vesicles (PL-EVs) constitute the major part of circulating EVs in the blood. They are also present in platelet concentrates (PCs) and may influence the quality of PCs, as recent reports implicate significant pro-thrombotic and pro-coagulant activities for PL-EVs. The aim of the present study was to analyze PL-EVs in PCs and correlate them with standard quality control (QC) parameters of PCs (e.g. functional capacity of platelets, duration of hemapheresis) and with donor-specific laboratory parameters (e.g. BMI, immature platelet fraction).

Methods: PL-EVs, shed from activated and senescent platelets, were analyzed by nanoparticle tracking analysis (NTA), and standard as well as advanced high sensitivity (Apogee 50M) flow cytometry. Platelet count and immature platelet fraction (IPF) were determined by a hematology analyzer. Functional capacity of platelets (i.e. externalization of CD62P in response to standard TRAP-6 activation) was measured by flow cytometry. Standard QC parameters were recorded during platelet hemapheresis. All in vitro measurements were carried out on day 0 (PC-production, 'fresh platelets') and on day 5 (after PC-expiry, 'senescent platelets') Altogether $n=42$ PCs were investigated, 15 of them were irradiated with 25 Gy on day 0.

Results: The externalization of CD62P, indicative for intact platelet function in PCs, significantly decreased during in vitro platelet senescence, and this was inversely correlated with the significant increase of PL-EVs/ platelets. The size of PL-EVs varied in all PCs investigated, ranging from approx. 70-550 nm, suggesting that diverse PL-EVs derive from different mechanisms. Interestingly, in fresh PCs a significant correlation was found between PL-EVs and PC-production with different hemapheresis devices, duration of platelet apheresis, and the IPF count in peripheral blood of the donor prior to apheresis. In senescent PCs the BMI of donors inversely correlated to the PL-EV-counts.
Conclusion: Accurate measurement of PL-EVs is highly recommended in the regular QC of PCs as a plausibility check for platelet function. Shedding of PL-EVs in PCs depends on shear stress in hemapheresis and diverse pre-analytical conditions.

\section{HQK-P12}

\section{Identification of blood donors with IgA deficiency for the provision of fresh frozen plasma with reduced allergic potential}

\section{${ }^{*}$ G. H. W. Hersmann, C. Günther, M. Koburger, K. Linke, H. Kroll} Inst.f.Transfusionsmedizin Suhl, Suhl, Germany

Background: Patients with immunoglobulin A ( $\operatorname{IgA}$ A deficiency are threatened by severe anaphylactic transfusion reactions when treated with plasma containing blood products. Therefore, patients at risk should only receive plasma or platelets from donors with very low level or absent IgA. In order to provide suitable plasma for transfusion purposes we screened our donor population for $\operatorname{IgA}$ and anti-IgA with different methods.

Methods: From December 2007 until April 2013 IgA concentrations were analyzed in blood donors who qualified for plasmapheresis. The IgA plasma concentration was measured by turbidimetry (Abbott IgA test, Architect, Aeroset). In selected cases, confirmation testing was performed by immuno nephelometry (Beckman Coulter). Selected donors with IgA concentrations below $0.3 \mathrm{~g} / 1$ were further analyzed in particle agglutination assay (ID-PaGIA IgA, BioRad). Antibodies against IgA were detected by ID-PaGIA-Anti-IgA test (BioRad).

Results: Of 7,416 blood donors, $32(0.43 \%)$ had an IgA concentration below $0.3 \mathrm{~g} / \mathrm{l}$ in turbidimetric assay. 17 randomly selected samples were confirmed by nephelometry. 32 donors with IgA concentrations below 0.3 $\mathrm{g} / \mathrm{l}$ were investigated in particle agglutination: Of 19 donors with very low IgA concentrations $(<0.001 \mathrm{~g} / \mathrm{l}), 15$ showed negative results and four showed positive results in particle agglutination. 13 donors had IgA concentrations between 0.1 and $0.3 \mathrm{~g} / \mathrm{l}$, eleven of whom were positive and two were negative in agglutination assay. Two of 15 IgA-deficiency donors were found to have Anti-IgA.

Conclusion: Here, we could demonstrate that $0.26 \%(19$ of 7,416$)$ of our blood donors had IgA levels below $0.001 \mathrm{~g} / 1$ and, therefore, were suitable as IgA deficient plasma donors. The results of the particle agglutination assay did not completely correlate with those obtained by turbidimetric test. IgA antibodies were found in $0.027 \%$ of the donors. Our institution is now capable to supply fresh frozen plasma for IgA deficient patients.

\section{HQK-P13}

\section{Influence of different preparation methods and storage solutions on platelet functional capacity in vitro}

\author{
${ }^{*}$ W. Madla1, G. Walther-Wenke ${ }^{2}$, R. Deitenbeck ${ }^{3}$, A. Grolle ${ }^{3}$, M. \\ Brodde $^{4}$, B. Kehrel', J. Bux ${ }^{3}$ \\ ${ }^{1}$ DRK Blutspendedienst West, Zentrale Herstellungsleitung, Hagen, \\ Germany \\ 2DRK Blutspendedienst West, Münster, Germany \\ ${ }^{3}$ DRK Blutspendedienst West, Hagen, Germany \\ ${ }^{4}$ OxProtect, Münster, Germany
}

Background: The automated preparation of blood products ultimately aims at achieving greater process efficiency and quality standardization; hence the use of the OrbiSac (O) or TACSI (T) systems for obtaining leucocyte-depleted platelet concentrates (O-PC, T-PC) from pooled buffy coats. This study investigates the effect of these technologies on selected quality criteria - in this case the potential for platelet (PLT) activation compared to the standard manual method (M-PC). We also assessed to what extent the parameters in question were affected by various storage solutions.

Methods: Six PCs per preparation method (pooling of 4 buffy coats for $\mathrm{M}-\mathrm{PC}, \mathrm{O}-\mathrm{PC}, \mathrm{T}-\mathrm{PC}$ ) and storage solution (plasma (PL), additive solutions (AS) SSP, SSP+) were analyzed by flow cytometry after $0 \mathrm{~h}, 24 \mathrm{~h}$ and 72 
$\mathrm{h}$ in order to assess the following in vitro parameters: ADP- and thrombin-induced (FIIa-induced) fibrinogen binding, FIIa-induced expression of CD62P and CD63, FIIa-induced cleavage of the PAR-1 receptor. The results of a total of 9 different $\mathrm{PC}$ preparations were compared with each other and with the results of platelets freshly obtained from whole blood. Results: ADP only achieved a poor level of PLT activation in all preparations; fibrinogen binding was strongest in the PL PCs. There were few differences between the methods. The results obtained from FIIa-induced fibrinogen binding were comparable to those of ADP, though the PLT did reveal a greater potential for activation. Here too there was evidence of significantly better fibrinogen binding in PL PCs. FIIa-induced CD62P expression was clearly pronounced in all preparations, and tended to be better with PL and M-PCs. Even after 72 hours the PLT of all PCs displayed good CD63 antibody binding. PL PCs exhibited the best response after the addition of thrombin. The use of SSP + effected a significantly better response from O-PC than from T-PC. Data on FIIa-induced cleavage of the PAR-1 receptor provide evidence of an equally intact thrombin receptor in all preparations at all times.

Conclusion: The examination of PLT functional capacity indicated that no advantage is gained by exchanging plasma for AS, and that $\mathrm{SSP}+$ in turn is preferable to SSP. ADP- and thrombin-induced binding of fibrinogen in particular were found to suffer negative effects from storage. The other parameters, however, indicated that cell viability in the PCs remained unchanged. Unlike the examination of baseline platelet activation, the present data revealed differences between the preparation methods which to some extent were significant, with better values in individual cases for O-PC vs T-PC.

\section{HQK-P14}

\section{Evaluation of different automated detection systems for microbiological control of cellular products}

\author{
*U. Schurig \\ Paul-Ehrlich-Institut, Bakteriologische Sicherheit, Langen, Germany
}

Background: According to Ph. Eur. Chapter 2.6.27, automated culturing systems may be used for microbiological control of cellular products. Growth detection is usually performed by continuous readout of metabolic parameters. Automated systems are intended and validated for the detection of infectious agents from patients' whole blood. Validation performed by the applicant is necessary when those systems are used for different types of therapeutic preparations.

Two automated detection systems (BACTEC; Becton Dickinson and BacT/Alert; Biomerieux) were compared to show their suitability for quality control in principal.

Methods: 13 microbial strains were used in this approach: those required by $\mathrm{Ph}$. Eur. Chapters 2.6.1 and 2.6.27 for growth promotion, method suitability or validation; environmental species; species isolated from skin and contaminants of blood components.

Strains were inoculated with bacterial counts of 10-30 CFU per bottle for growth promotion test according to $\mathrm{Ph}$. Eur. requirements. In both $\mathrm{BAC}$ TEC and BacT/Alert, commercially available standard bottles without adsorbing substances or other supplements were used (BACTEC Standard Aerobic / Anaerobic; BacT/Alert iAST / iNST bottles). Incubation temperature in BACTEC and BacT/Alert was $32,5^{\circ} \mathrm{C}$. Four strains were evaluated for detection by automated systems in the presence of hematopoietic stem cells ( 5 different donors, from peripheral blood). Seven other microbial strains were tested in presence of two stem cell preparations.

Results: Media evaluation showed good compliance of both automated systems. Differences were observed in anaerobic bottles for strictly aerobic or anaerobic micro-organisms. B. subtilis, Ps. aeruginosa, Ps. fluorescens, Micrococcus sp, C. albicans and A. brasiliensis were not detected in BACTEC anaerobic bottles, but in BacT/Alert iNST bottles only Ps. fluorescens showed no growth and A. brasiliensis was detected irregularly. Detection of $C$. sporogenes occurred later in BacT/Alert iNST than in BACTEC Standard anaerobic bottles.
Matrix evaluation in presence of stem cell preparations showed good compliance in both automated systems. With 3 of the 5 tested preparations, growth was detected within 10 and 25 hours. Longer detection times could be found for $A$. brasiliensis and $P$. acnes and greater differences between the two automated systems for $C$. sporogenes. With two preparations, growth of the strains was detected only irregularly.

Conclusion: Both automated systems were suitable to detect growth of the tested micro-organisms, but conditions in anaerobic bottles are different in comparison with inoculation in thioglycolat. Inoculation of BacT/ Alert Anaerobic bottles has to be performed very careful in order to maintain the anaerobic conditions. BACTEC Anaerobic bottles keep strong anaerobic conditions.

In stem cell preparations, donor characteristics may influence the outcome of a validation. Additional studies are necessary to investigate the background of this observation.

\section{HQK-P15}

\section{Comparison of growth and time to detection of different microbial strains in dependence of incubation temperature in an automated detection system}

\section{*U. Schurig \\ Paul-Ehrlich-Institut, Bakteriologische Sicherheit, Langen, Germany}

Background: Microbiological control of cellular products is performed according to the requirements of $\mathrm{Ph}$. Eur. Chapter 2.6.27, currently at an incubation temperature of 35 to $37^{\circ} \mathrm{C}$. This temperature is optimal for mesophilic microbial species, represented for instance by clinical isolates, but not for psychrotolerant environmental microbial species. Micro-organisms may be present in the source material or contaminate cells and tissues during procurement or at manufacturing. When different conditions are applied, some species may grow in the product itself, but not in the culturing medium because of the higher incubation temperature. Methods: In BacT/Alert 3D Dual-T (Biomerieux), incubation of $17 \mathrm{mi}$ crobial strains was performed at $22,5^{\circ} \mathrm{C}$ and $32,5^{\circ} \mathrm{C}$ in BacT/Alert iAST aerobic bottles and at $32,5^{\circ} \mathrm{C}$ in iNST anaerobic bottles with or without stem cell preparations. Strains used are either required by $\mathrm{Ph}$. Eur. Chapter 2.6.1 or 2.6.27 for growth promotion and method validation; belong to skin and environmental strains or WHO transfusion relevant bacterial reference (TRBR) strains by PEI. Some of the strains were incubated additionally at $35^{\circ} \mathrm{C}$. The strains were inoculated with bacterial counts of around 10 to $30 \mathrm{CFU}$ per bottle.

Results: Most of the tested micro-organisms showed shorter detection time at higher temperatures. In comparison with $32,5^{\circ} \mathrm{C}$, detection at 22,5 ${ }^{\circ} \mathrm{C}$ was delayed for 20 hours in the case of B. subtilis, Ps. aeruginosa, $C$. albicans, Str. pyogenes and K. pneumoniae. For some other strains, such as St. aureus, Micrococcus sp., A. brasiliensis, St. epidermidis and E. coli, a delay in detection between 35 and about 50 hours was observed at 22,5 ${ }^{\circ}$ C. Ps. fluorescens and Penicillium sp. showed only a few hours delay at $22,5^{\circ} \mathrm{C}$. On the contrary, Cladosporium cladosporoides was not able to grow at $32,5^{\circ} \mathrm{C}$, but could be detected only at $22,5^{\circ} \mathrm{C}$.

The comparison between the incubation at $32,5^{\circ} \mathrm{C}$ and $35^{\circ} \mathrm{C}$ showed on the one hand shorter detection times especially for Staphylococcus $s p$. and Propionibacterium acnes, but on the other hand no detection for PS. fluorescens and Sphingomonas sp.

Conclusion: The currently used one temperature of $35^{\circ} \mathrm{C}$ used for testing should be extended to two temperatures in order to cover the most relevant temperatures at manufacturing and storage. Applied temperatures should meet the requirements of relevant micro-organisms for the type of product in order to provide the best conditions and shortest time to detection.

$35^{\circ} \mathrm{C}$ is a suitable incubation temperature for anaerobic testing, but aerobic testing should be performed preferably at $22,5^{\circ} \mathrm{C}$ and $32,5^{\circ} \mathrm{C}$. Optimal conditions for strictly aerobic contaminants cannot be provided by the anaerobic bottles and are not met by the lower temperature only. 


\section{HQK-P16}

\section{Correlation of the hypotonic shock response and exchange of shape change with the new ThromboLUX ${ }^{\circledR}$}

\section{Krämer, T. Raczat, J. Strobel, D.R. Weiß, R. Eckstein, *J. Ringwald}

Universitätsklinikum Erlangen, Transfusionsmedizin und Hämostaseologie, Erlangen, Germany

Background: ThromboLUX ${ }^{\circledR}$ (LightIntegra, Vancouver, Canada) uses dynamic light scattering for analysing kind and number of microparticles and the response of platelets (PLTs) to temperature stress. Paired comparison of this new in-vitro quality parameter with established standard tests such as the hypotonic shock response (HSR) or the extent of shape change (ESC) are rare. We performed a paired comparison of ThromboLUX (TLX) Score with HSR and ESC in samples derived from single donor platelet concentrates (SDPs).

Methods: Samples were taken from SDPs stored between one and seven days which had not been used for transfusion for different reasons. Either they had been expired after four days of storage as they had not been needed for transfusion or they had not been released for transfusion due to visible aggregates, a content of more than $1 \times 10^{6}$ white blood cells per unit or increased liver enzymes of the donor. One SDP could have been tested on different days and all tests were performed in parallel and in duplicate. Mean of the two tests was used for analysis. For HSR and ESC testing, Chrono-Log SPA2000 (Chrono-Log,Havertown,PA) was used and platelet rich plasma was adjusted to a concentration of 200 to 300 PLTs per nL. Correlation between TLX Score, HSR and ESC was tested using Pearson's correlation coefficient for all SDPs together and for SDPs of different age.

Results: We performed testing of 99 samples derived from 42 SDPs. Mean values for TLX Score, HSR and ESC was $30.3+3.8,69.0+12.2 \%$, and $23.2+4.9 \%$, respectively. Correlation testing of all SDPs revealed no significant correlation between TLX Score and HSR or ESC ( $r=-0.158$, $\mathrm{p}=0.118$ and $\mathrm{r}=-115, \mathrm{p}=0.255$, respectively) whereas HSR and ESC correlated significantly $(r=0.351, p<0.001)$. Additionally, the data were analysed separately for SDPs stored up to or longer than 4 days. Interestingly, we found significant positive correlation between TLX Score and ESC as well as between HSR and ESC only for SDPs stored 5 to 7 days but not for SDPs stored up to 4 days.

Conclusion: Overall, the new PLT in-vitro quality parameter TLX Score did not show significant correlation with the two standard in-vitro parameters HSR and ESC except for outdated SDPs with regard to TLX Score and ESC. However, further studies are needed testing all three in-vitro quality parameters against PLT transfusion outcome to get valid information about their predictive value for the success of PLT transfusion.

\section{HQK-P17}

Distribution and supply of RBCs by Blood Donation Service West Centres for Transfusion Medicine Münster and Bad Salzuflen, Germany

\section{${ }^{*} D$. Kühnel', B. Spier ${ }^{1}, T$. Alt ${ }^{2}$, W. Engel ${ }^{3}$, G. Walther- Wenke ${ }^{1}$ ${ }^{1}$ DRK BSD West Zentrum für Transfusionsmedizin, Münster, Germany ${ }^{2}$ DRK BSD Rheinland-Pfalz/Saarland, Bad Kreuznach, Germany ${ }^{3}$ DRK BSD Ostwestfalen- Lippe, Bad Salzuflen, Germany}

Background: The Transfusion Medicine Centers Münster and Bad Salzuflen are located in North-Rhine-Westphalia, with a catchment area of app. 4.63 million inhabitants. According to the population based distribution of bloodgroups in Germany [O pos. $35 \%$, A pos. $37 \%$, B pos. $9 \%$, AB pos. $4 \%$, O neg. $6 \%$ A neg. $6 \%$ B neg. $2 \%$, AB neg. $1 \%$; Lit.: Thomas, L.: Labor und Diagnose, 1992] we supply our regional hospitals. The most important blood groups in transfusion are $\mathrm{ABO}$ and $\mathrm{Rh}$ blood group system. $6 \%$ of the population in Germany are blood group $\mathrm{O} \mathrm{RhD}$ negative. Methods: In a retrospective analysis a statistical evaluation of produced RBCs in 2002, 2007 and 2012 (proper for transfusion) and their ABO and $\mathrm{Rh}$ blood groups was performed.
Results: There is a clinical and discontinuous distribution. O RhD neg. blood group is relatively rare, known as "universal donor" blood group and very important in emergency situations. There are mandatory, recommended and acceptable indications for the transfusion of $\mathrm{O} \mathrm{RhD}$ negative red blood cells. Our blood donation service produced more than $8 \%$ of RBCs to be group O RhD negative to meet demand from hospitals (Fig.1). By comparing the years 2002, 2007, 2012 (Tab.1) we see a significant variation in collecting blood from blood donors with blood group O neg. $\left(X^{2}=111.81 ; p=2.4905 E-17\right)$. According to the demographic changes in Germany our collection numbers differed in the investigated years. In the last years we increased our activities in personal contact with our blood donors. The statistical differences of RBCs/ donors/ year between 2002/2007 and 2007/2012 are highly significant (Mann-Whitney-U-Test:p < 0.001)

Conclusion: To summarise, we determined that the motivation to donate blood regularly is subject to many factors. By targeted personal contact (letter, telephon, email etc.) a change in donor-behaviour is possible. When differentiating donation rates according to the investigated years, we see an effect of our efforts. The blood supply of the regional hospitals also for rare blood groups (e.g. O RhD negative) is secured. The changes in blood recipient and donor population will be challenges in the future.

Fig. 1: Distribution of O RhD negatve RBCs/month in 2002, 2007, 2012

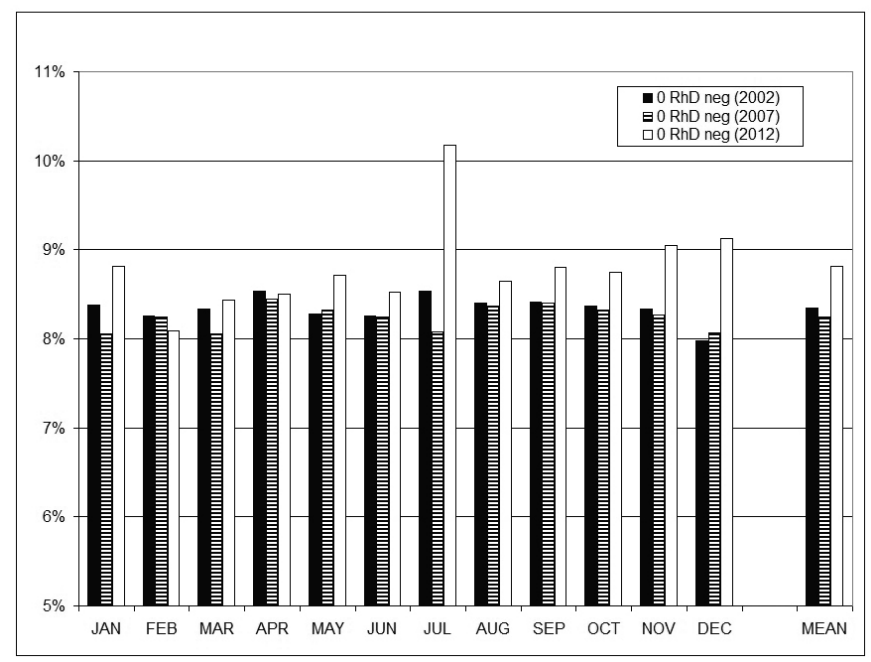


HQK-P18

\section{Occurence of residual platelet aggregates in single donor platelet concentrates in routine blood banking}

\author{
*J. Ringwald"1, R. Eckstein 1 , M. Antoon ${ }^{2}$, M. Cardoso ${ }^{2}$ \\ ${ }^{1}$ Universitätsklinikum Erlangen, Transfusionsmedizin und \\ Hämostaseologie, Erlangen, Germany \\ ${ }^{2}$ TerumoBCT, Zaventem, Belgium
}

Background: Platelet (PLT) aggregates (PAs) can occur during processing of whole-blood or apheresis derived PLT components. Collection of single donor PLT concentrates (SDPs) at higher concentrations and yields may increase the likelihood of PAs. Most of them disperse with rest periods and agitation, but in some rare cases PAs persist for more than $24 \mathrm{~h}$ and may prevent release of the product. We retrospectively analyzed the presence of PAs in SDPs stored in $100 \%$ plasma and their impact on product release. Furthermore, we aimed to detect variables being associated with PA occurrence. Methods: We studied the production protocols of 803 SDPs collected on TRIMA ACCEL (TerumoBCT) or AMICUS (Fenwal) during randomly chosen periods in 2012. All SDPs are checked for the appearance of macroscopically visible PAs by an operator within $1 \mathrm{~h}$ after production (T1) and, if positive, again by a physician 6 to $24 \mathrm{~h}$ later (T2). Additionally, in all SDPs residual white blood cells (WBCs) are determined with Nageotte chamber and microscopically visible PAs are documented. SDPs with persisting macroscopically visible PAs should not be released.

Results: Macroscopically or microscopically visible PAs were initially present 12.5 and $8.7 \%$, respectively. Macroscopically visible PAs disappeared within $24 \mathrm{~h}$ in $81 \%$. Eleven units with positive PAs at T1 had not been checked at T2. Ten units with PAs were not released resulting in a total discard rate of $1.2 \%$ due to PAs. SDPs with or without PAs did not differ in variables such as separation volume, collection time, anticoagulant volume in SDPs, donor's total blood volume, WBC or PLT count However, SDPs with PAs were donated more often by females and donors with lower hematocrit. For double collection with TRIMA ACCEL, SDPs with PAs also showed a higher PLT concentration and content.

Conclusion: PAs are present in routine SDP production to a relevant extent but the vast majority disappears within $24 \mathrm{~h}$. The loss of SDPs due to persistent PAs is rather low. The relevance of the higher rate of females and individuals with lower hematocrit among the donors of SDPs with PAs as well as the clinical impact of the phenomenon needs further prospective in-vitro and in-vivo studies.

\section{Oral Abstract Session: Immunhämatologie}

\section{$\mathrm{IMH}-\mathrm{V} 01$}

Recombinant blood group proteins facilitate the detection of alloantibodies to high-prevalence antigens and uncover hidden antibodies: results of an international study

${ }^{*}$ A. Seltsam ${ }^{1}$, F. Wagner', M. Lambert', T. Bullock ${ }^{3}$, N. Thornton ${ }^{3}$, E. A. Scharberg 4 , D. Grueger ${ }^{5}$, C. Schneeweiß ${ }^{5}$, R. Blasczyk ${ }^{6}$

${ }^{1}$ German Red Cross Blood Service NSTOB, Institute Springe, Springe, Germany

${ }^{2}$ National Blood Service, Irish Blood Transfusion Service, Dublin, Ireland ${ }^{3} \mathrm{NHSBT}$ Filton Blood Centre, International Blood Group Reference Laboratory, Bristol, United Kingdom

${ }^{4}$ German Red Cross Blood Service Baden-Württemberg-Hessen, Institute for Transfusion Medicine and Immunohematology, Baden-Baden, Germany ${ }^{5}$ Imusyn, Hannover, Germany

${ }^{6}$ Hannover Medical School, Institute for Transfusion Medicine, Hannover, Germany

Background: Red blood cell (RBC) alloantibodies to high-prevalence antigens (hp-AGs) are not easily specified by routine antibody identification tests using standard test cell panels. Thus, occurrence of alloan- tibodies to hp-AGs generally leads to a delay in antibody identification and a possible delay in patient care. Recombinant blood group proteins (rBGPs) are useful for identification of such antibodies. This multicenter study was conducted to test the use of rBGPs in regional and international $\mathrm{RBC}$ reference laboratories.

Methods: For a period of 6 months, single soluble rBGPs (Lu, Yt, Kn (CR1, LHR-D), JMH, Sc, Rg, Ch, Do, Cr) or cocktails of them were assessed for their ability to inhibit reactivity of antibodies to the corresponding antibody specificities. Four different national and international reference laboratories took part in the study. Initially, the use of rBGPs was validated by testing panels of well characterized patient serum samples containing antibodies to hp-AGs in the inhibition assay. Then, the rBGPs were prospectively used in all participating laboratories for routine antibody identification and the results were compared to those obtained with RBCs-based diagnostics.

Results: Panels of predefined antibodies to hp-AGs $(\mathrm{N}=40)$ were completely and specifically neutralized by the corresponding rBGP specificities. The rBGPs were used prospectively for antibody identification in a total of 81 cases. Antibodies to hp-AGs were specifically inhibited by the corresponding rBGP specificities except for some CR1-related antibodies $(\mathrm{N}=6)$ which may be directed to so far undefined epitopes not expressed on the truncated recombinant CR1. In five cases, more than $1 \mu \mathrm{g}$ of rBGP was required to achieve full inhibition. In 13 cases, one $(\mathrm{N}=5)$ or multiple $(\mathrm{N}=8)$ additional clinically relevant alloantibodies could clearly be identified in the presence of an antibody to a hp-AG. The rBGPs were successfully used for crossmatching to inhibit the reactivity of a clinically irrelevant antibody to a hp-AG, to determine compatibility between donor and recipient.

Conclusion: Soluble rBGPs are stable and can be easily integrated into routine serology. rBGP-based diagnostics enables the identification of antibodies to hp-AGs without the need for rare $\mathrm{RBC}$ reagents which are often unavailable. Underlying antibodies can be reliably detected and crossmatching results be validated in the presence of antibodies to hp-AGs. Because of their capacity for single-step, direct antibody determination, rBGP-assays will greatly facilitate and accelerate the process of antibody identification, resulting in a more efficient blood supply for immunized patients.

\section{IMH-V02}

Top versus bottom RBC sample drawing for blood group typing: analysis of mixed fields in solid phase and column agglutination

\section{${ }^{*}$ S. Flommersfeld, B. Ertl, G. Bein, U. J. Sachs}

Universtitätsklinikum Marburg, Transfusionsmedizin, Marburg, Germany

Background: The recognition of mixed fields (MF) sometimes hampers proper blood group typing in patients with a history of recent RBC transfusions. Several case reports have claimed that in automated blood group typing, mixed fields may occur more frequently, since in automatic devices, RBCs are pipetted from the bottom of the test tube. Transfused RBCs are supposed to be preferably present at the bottom because they gain weight during shelf storage and may thus settle faster than autologous cells.

Methods: During a time period of four weeks, we identified 22 patients who were transfused with RBCs incompatible for CcEe or K due to shortage of fully compatible RBCs. CcEe and $\mathrm{K}$ typing was performed on the latest available post-transfusion blood sample manually (using column agglutination from BioRad, Dreieich, Germany) or automatically (using the solid phase system GalileoNEO from Immucor, Rödermark, Germany). RBCs were collected from the top and bottom parts of the test tube. Results: Mixed fields were identified in 20/22 patients (30/37 antigen discrepancies) by column agglutination (CA) and in 14/22 patients (17/37 antigen discrepancies) in the automated solid phase (SP) assay. Findings between top and bottom samples differed in $6 / 22$ patients in both systems (antigen discrepancies, 12/37 for CA and 8/37 for SP). CA detected 4 antigen discrepancies in the top and 8 in the bottom sample; SP detected 
5 antigen discrepancies in the top and 3 in the bottom sample. The mean age of transfused RBCs in samples with MF in the top sample was 11 days, but was 19 days for those with MF in the bottom sample $(\mathrm{p}=0.004)$ Conclusion: The SP assay is less sensitive to recent transfusions compared to CA. Since all samples in which mixed field were not detected by SP were typed correctly, this could be an advantage of the SP assay in clinical routine. If, however, identification of previous transfusions is warranted, CA should be used preferably (sensitivity approx. 90\% vs. $64 \%$ ). In contrast to previous reports, transfused RBCs are not regularly found on the bottom of the test tube. We found evidence that this phenomenon might increase with the age of the transfused RBC. Again, SP was more robust than $\mathrm{CA}$ in typing $\mathrm{CcEe}$ and $\mathrm{K}$ from these samples.

\section{IMH-V03}

\section{Adsorption-elution dependent confirmation of RhD positivity expressed by the large hybrid-allele RHD-CE(4-9)-D}

\section{S. Meyer ${ }^{1}$, K. Neuenschwander ${ }^{1}$, S. Sigurdardottir ${ }^{1}$, J. Gottschalk², T. Schulzki ${ }^{3}$, T. Weingand ${ }^{4}$, A. Maier ${ }^{4}$, B. M. Frey ${ }^{5}$, ${ }^{*}$ C. Gassner ${ }^{1}$}

${ }^{1}$ Blood Transfusion Service Zurich, Swiss Red Cross, Molecular Diagnostics \& Cytometry, Schlieren, Switzerland, Switzerland

${ }^{2}$ Blood Transfusion Service Zurich, Swiss Red Cross, Screening, Schlieren, Switzerland, Switzerland

${ }^{3}$ Blood Transfusion Service Graubünden, Swiss Red Cross, Chur, Switzerland, Switzerland

${ }^{4}$ Blood Transfusion Service Central Switzerland, Swiss Red Cross, Luzern, Switzerland, Switzerland

${ }^{5}$ Blood Transfusion Service Zurich, Swiss Red Cross, Schlieren, Switzerland, Switzerland

Background: "Small" RHD-CE-D hybrid-alleles still code for some RhD epitopes and consequently appear serologically as "partial" RhDs, e.g. RhD VI. In contrast, "large" RHD-CE-D hybrid-alleles lack almost all RhD coding nucleotides (nt) and therefore generally appear as RhD negative. Since RHD-CE(4-7)-D (ISBT: $R H D * 01 N .07$ ) consistently reacts $\mathrm{RhD}$ negative by serology, accordingly, the hybrid-allele $R H D-C E(4-9)-D$, with an even larger $\mathrm{CE}$ insert, would be expected to be $\mathrm{RhD}$ negative as well.

Methods: Screening for $R H D$ among $\mathrm{RhD}$ negative blood donor samples was done using an in house validated method to detect the presence of $R H D$ exon 5 (combined nts $676 \mathrm{G}$ with $787 \mathrm{G}$ ), exon 7 (1048G) and the 3' UTR after exon 10 (nt 1359A) on single donor DNAs with RhD negativity. Molecular allele discrimination was done with commercially available kits, suitable (1) to scan for the presence of RHD gene exons by Sequence-Specific Priming (PCR-SSP), and (2) to detect the type(s) of "Rhesus boxes" (innotrain GmbH, Kronberg i.T., Germany). Phenotypic expression of residual $\mathrm{RhD}$ was assessed by adsorption-elution technique using polyclonal Anti-D antiserum.

Results: One single sample of $R H D-C E(4-9)-D \mid R H d$ (deletion) genotype was observed among 5'854 RhD negative blood donors. Adsorption-elution of Anti-D onto the red blood cells (RBC) of the propositus repetitively confirmed the expression of weak RhD on his RBC membrane. $R H D C E$-, and Rhesus box analysis-PCR-SSP revealed a heterozygous presence of a RHD-CE(4-9)-D large hybrid-allele in combination with a RhD negative $R H d$ (deletion) haplotype.

Conclusion: The observed $R H D-C E(4-9)-D$ hybrid allele exhibited weak $\mathrm{RhD}$ expression on the RBC surface, confirming a similar observation made in China. Therefore, RhD negativity of all large $R H D$-CE-D hybrid alleles may not be concluded in general. In the observed sample, presence of RHD specific nucleotides within the exon 4-9 CE-specific sequence hybridized into $R H D$, or on one of the two $R H C E$ genes must be postulated, but did not become apparent by PCR-SSP analysis. Large RHD-CE-D hybrid-alleles need (1) accurate identification, and/or (2) adsorption-elution driven $\mathrm{RhD}$ negativity confirmation, before their $\mathrm{RhD}$ negative status may be infered.

\section{IMH-V04 \\ Screening of patients for preexisting antibodies to pathogen inactivated red blood cells}

\section{${ }^{*}$ C. Geisen 1 , V. Brixner ${ }^{1}$, L. Stempniewski ${ }^{1}$, A. North ${ }^{2}$,} A.-H. Kiessling ${ }^{3}$, M.M. Müller ${ }^{1}$, N. Mufti ${ }^{2}$, E. Seifried ${ }^{1}$

${ }^{1}$ Institut für Transfusionsmedizin und Immunhämatologie; Universität

Frankfurt, Abteilung für Immunhämatologie, Hämostaseologie und Molekulare Diagnostik, Frankfurt/Main, Germany

${ }^{2}$ Cerus Corporation, Concord, CA, USA, Germany

${ }^{3}$ Universitätsklinik Frankfurt, Thorax-, Herz-, Thorakale Gefäßchirurgie,

Frankfurt/Main, Germany

Background: In preparation for a Phase 3 clinical trial using the S-303 Treatment System for pathogen inactivation of red blood cell components (RBCs) for transfusion of acute anemia patients, we screened more than 5000 patients in a large university hospital for pre-existing immune reactivity to S-303 treated RBCs. Prior experience with the first generation S-303 Treatment System had shown that there was a small proportion of patients ( 1 to $2 \%$ ) with pre-existing antibodies against the acridine moiety of S-303 that was associated with S-303 treated RBCs.

Methods: $\mathrm{RBC}$ of a voluntary blood group $\mathrm{O}$ blood donor were split into three parts and pathogen inactivated using 1) first generation PI method (high S-303 adducts) 2) second generation PI method (low S-303 adducts) and 3) not pathogen inactivated. RBC were frozen in Glycerolyte (Fenwal) and stored at $-80^{\circ} \mathrm{C}$ until day of antibody screening using a gel card centrifugation technique (BioRad). Patient samples were either stored at $4{ }^{\circ} \mathrm{C}$ or centrifuged and plasma was frozen at $-30^{\circ} \mathrm{C}$ within $48 \mathrm{~h}$ after blood drawing. For screening purposes, 25 $\mu \mathrm{l}$ of each patient sample was incubated with $50 \mu \mathrm{l}$ of $0.8 \%$ erythrocyte suspension and Liss/Coombs sera on DiaMed Gel Cards ${ }^{\circledR}$ using a Tecan Liquid handling machine. After $15 \mathrm{~min}$ of incubation at $37^{\circ} \mathrm{C}$ gel cards were centrifuged and checked for positive reactions using Saxo Reader (Biorad). A positive control of monoclonal antibody to S-303 was included for each run. Results: We analysed our potential study cohort for epidemiologic parameters and found this patient cohort to be of average age of 67 years (1 day to 99 years old) and to have a male / female ratio of $57 \%$ to $43 \%$. In one patient, immune reactivity to S-303 treated RBCs was detected and confirmed by inhibition of agglutination with an S-303 analogue, S-300. Further characterization revealed a warm reactive acridine-specific IgG-antibody with a titer of 4 against RBC with high acridine (first generation PI method). Reaction against low acridine cells (second generation PI method) was only weakly positive. The patient is a 73 year old male of Arabic descent undergoing arterial valve repair surgery. In all other patients acridine specific antibodies were excluded.

Conclusion: This study was designed to evaluate the prevalence of immune reactivity to S-303 treated RBCs in the planned clinical study cohort using the second generation S-303 treatment system that results in a reduced number of S-303 adducts per RBC. The prevalence of immune reactivity to S-303 treated RBCs using the second generation S-303 treatment process is expected to be a very rare event. Further studies are needed to reveal the prevalence of immune reactivity and acridine specific antibodies to S-303 treated RBCs in various patient cohorts.

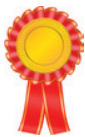

IMH-V05

CD59 defines a new blood group antigen

${ }^{*}$ M. Anliker ${ }^{1}$, I. von Zabern ${ }^{1}$, B. Höchsmann ${ }^{1}$, H. Kyrieleis ${ }^{2}$, C. Dohna-Schwake ${ }^{3}$, H. Schrezenmeier ${ }^{1}$, C. Weinstock ${ }^{1}$

${ }^{1}$ Institute of Clinical Transfusion Medicine and Immunogenetics UIm, German Red Cross Blood Service Baden-Württemberg - Hessen, Institute UIm, University of UIm, UIm, Germany

${ }^{2}$ Hospital Bethanien, Department of Pediatrics, Moers, Germany

${ }^{3}$ University Hospital of Essen, Department of Pediatrics, Essen, Germany

Background: Although CD59 is known since a long time as a constituent of the erythrocyte membrane, it so far has not been defined as an blood 
group antigen. Homologous restriction factor (HRF), membrane inhibitor of reactive lysis (MIRL) and membrane attack complex inhibitory factor (MACIF) are further designations for this glycoprotein that limits complement lysis. Six cases of an isolated deficiency of CD59 with two molecular bases have been published so far (Motoyama, M et al, 1992; Nevo, Y et al., 2012). We recently described the new CD59 allele c.146delA discovered in a young child of Turkish origin (Höchsmann B. et al., 2010, DOI:10.1159/000321410).

Methods: Here, we give evidence for the presence of an alloantibody directed against CD59 in the plasma of this child. We also present easily applicable methods of identification for this antibody. Serologic testing was performed using a gel matrix test to detect agglutination. CD59 and CD55 were determined with FITC labelled anti-human-CD59 and PE labelled anti-human-CD55 (Becton Dickinson Pharmingen). His tagged and non-glycosylated recombinant soluble CD59 protein was obtained in lyophilized form (Creative Bio Mart) In the plasma of the child carrying a homozygous isolated CD59 deficiency, an antibody against a high frequency antigen was detected at the age of 18 months. Between the $2^{\text {nd }}$ and $3^{\text {rd }}$ years of age the antibody titer was 4.

Results: The reactivity of the antibody was enhanced by treatment of RBC with papain but was destroyed by treatment with DTT. To identify the specificity of the antibody, soluble recombinant anti-CD59 was added to the plasma of the CD59 deficient patient. As a control, monoclonal anti-CD59 and monoclonal anti-CD55 were used instead of plasma. Flow cytometric analysis demonstrated inhibition of the patient antibody and of commercial monoclonal anti-CD59 by the recombinant soluble CD59 protein while the reactivity of anti-CD55 remained unaffected. To design an assay even more easily to perform, we used the gel agglutination technique. Agglutination by the irregular antibody could be completely inhibited by preincubation of the plasma with recombinant CD59. In further experiments using the same test system, the alloantibodies anti-K, anti-C, anti-c or anti-Fy(a) were added to the plasma of the CD59 deficient patient.

Conclusion: While the antibody directed against the high frequency antigen CD59 was blocked, the admixed alloantibodies were still detectable. A CD59 deficient patient had formed an anti-CD59 antibody, easily detectable by blocking the antibody reactivity by recombinant soluble CD59. The CD59 deficiency was inherited from the parents who both were heterozygous for the same allele. The erythrocyte membrane glycoprotein CD59 is well characterized on a molecular level, but this is the first demonstration of a human antibody directed against it. Therefore, CD59 represents a candidate for a new blood group system.

\section{IMH-V06}

\section{Vel-negative allele frequency comparison in one Austrian and three Swiss regions for efficiency optimization of donor screening and recruitment}

\section{Jungbauer ${ }^{1}$, S. Sigurdardottir ${ }^{2}$, S. Meyer ${ }^{2}$, J. Gottschalk ${ }^{3}, T$. Schulzki ${ }^{4}$, T. Weingand 5 , A. Maier ${ }^{5}$, B.M. Frey ${ }^{6},{ }^{*}$ C. Gassner ${ }^{2}$}

${ }^{1}$ Blood Transfusion Service Wien, Niederösterreich \& Burgenland, Autrian Red Cross, Vienna, Austria, Austria

${ }^{2}$ Blood Transfusion Service Zurich, Swiss Red Cross, Molecular

Diagnostics \& Cytometry, Schlieren, Switzerland, Switzerland

${ }^{3}$ Blood Transfusion Service Zurich, Swiss Red Cross, Screening,

Schlieren, Switzerland, Switzerland

${ }^{4}$ Blood Transfusion Service Graubünden, Swiss Red Cross, Chur, Switzerland, Switzerland

${ }^{5}$ Blood Transfusion Service Central Switzerland, Swiss Red Cross, Luzern, Switzerland, Germany

${ }^{6}$ Blood Transfusion Service Zurich, Swiss Red Cross, Schlieren,

Switzerland, Switzerland

Background: The blood group Vel was discovered 60 years ago and individuals negative for this antigen are rare and are required for the safe transfusion of patients with antibodies to Vel. Very recently, the Vel-negative phenotype could be linked to SMIM1, located on chromosome $1 \mathrm{p} 36$ As reported three times timely in parallel, almost all Vel-negative individ- uals investigated so far (e.g. 164 of 169) were homozygous for a frameshift deletion of 17 bp in exon 3. An in house PCR using Sequence Specific Priming (PCR-SSP) was used for rapid Vel screening and genotyping. Methods: Austrian samples were from 422 blood donors of the Vienna region ("VI"), or were 855 DNAs collected in Switzerland in the course of the mandatory screening for the presence of $R H D$ among $\mathrm{RhD}$ negative donors. Swiss regions were roughly represented by the cantons of Graubünden ("GR"), Central Switzerland ("CE": Luzern, Nidwalden, Obwalden, Schwyz and Zug), and Zurich and Schaffhausen ("ZH”). Using data published, two separate PCR-SSPs, one specific for the recognition of the Vel-negative allele with the $17 \mathrm{bp}$ deletion (screening reaction) and another for the wildtype-allele were devised and positively validated using samples with known Vel phenotype.

Results: Of the Austrian and the three different Swiss regions of VI, GR, $\mathrm{CE}$, and $\mathrm{ZH}, 422,238,196$ and 421 individuals were screened and 13, 10,8 and 10 were heterozygous for the Vel-negative allele, respectively. Allele-frequencies for this allele were calculated to be $0.0154,0.0210$, 0.0204 and 0.0119 in VI, GR, CE, and ZH, and Vel-negative homozygous donors may consequently be expected at a frequency of 2.4, 4.4, 4.2 and 1.4 per 10'000 donors, respectively (table 1 ).

Conclusion: Genetic linkage of the Vel locus SMIM1 on chromosome 1 with the $25 \mathrm{Mbp}$ distanced $R H D$ may not be excluded a priori, and frequency data may therefore not be balanced among the investigated $\mathrm{RhD}$ negative Swiss donors, and additionally be incomparable to data obtained from $\mathrm{RhD}$ positive/negative Austrian donors. However, Vel-negative donor screening and recruitment would be more than three times more efficient in GR than in $\mathrm{ZH}$, expecting 4.4 versus 1.4 Vel-negative donors per 10 '000, respectively. Allocation of screening resources should therefore be done accordingly.

\begin{tabular}{|lccccc|}
\hline $\begin{array}{l}\text { Natio- } \\
\text { nality }\end{array}$ & Region & $\begin{array}{c}\text { n donor samples } \\
\text { investigated }\end{array}$ & $\begin{array}{c}\text { n Vel-negative } \\
\text { heterozygous }\end{array}$ & $\begin{array}{c}\text { Vel-negative } \\
\text { allele frequency }\end{array}$ & $\begin{array}{c}\text { expected Vel- } \\
\text { negative homo- } \\
\text { zygous per 10'000 }\end{array}$ \\
\hline AT & VI & 422 & 13 & 0.0154 & 2.4 \\
CH & GR & 238 & 10 & 0.0210 & 4.4 \\
CH & CE & 196 & 8 & 0.0204 & 4.2 \\
CH & ZH & 421 & 10 & 0.0119 & 1.4 \\
\hline TOTAL & $\mathbf{1} \mathbf{2 7 7 7}$ & $\mathbf{4 1}$ & $\mathbf{0 . 0 1 6 1}$ & $\mathbf{2 . 6}$ \\
\hline
\end{tabular}

\section{IMH-V07}

\section{Molecular screening for the Vel- blood group phenotype}

\section{${ }^{*}$ P. Bugert, G. Rink, H. Klüter, E. A. Scharberg}

DRK-Blutspendedienst Baden-Württemberg - Hessen, Institut für Transfusionsmedizin und Immunologie, Mannheim, Germany

Background: Vel is a high prevalence blood group antigen. Population studies estimate the prevalence of Vel- individuals at about 1 in 4,000 in Europe. Anti-Vel can cause severe hemolytic transfusion reactions and a hemolytic disease of the newborn. Recently, the molecular basis of the Vel- phenotype was discovered as a homozygous $17 \mathrm{bp}$ deletion in the coding region of the SMIMI gene. We developed PCR methods for the rapid detection of the SMIMI $17 \mathrm{bp}$ deletion and screened a German blood donor cohort.

Methods: PCR methods based on the use of sequence-specific primers (PCR-SSP) and on the use of TaqMan probes (TaqMan-PCR) were developed for detection of the wild-type (Vel+) and the deletional (Vel-) SMIM1 alleles. Both methods were validated using two serologically tested Vel- reference samples. Molecular screening in a blood donor cohort was performed by TaqMan-PCR. Selected samples with Vel+/+, Vel+/and Vel-/- genotypes were re-typed using PCR-SSP.

Results: The Vel- reference samples showed the expected genotype, i.e. homozygous 17 bp deletion in the SMIMI gene, both in PCR-SSP and TaqMan-PCR. Screening of 2,592 blood donors using the TaqManPCR method revealed 2,497 (96.33\%) Vel+/+, 94 (3.63\%) Vel+/- and 1 (0.04\%) Vel-/- individuals (allele frequencies: Vel+ $98.15 \%$; Vel- $1.85 \%$ ). 
Re-typing of all Vel+/- and Vel-/- samples as well as 22 Vel+/+ samples by PCR-SSP confirmed the TaqMan-PCR genotype results.

Conclusion: Our PCR methods enable a fast screening for Vel- blood donors and genotyping of patients in clinical diagnosis. TaqMan-PCR could be the method of choice for screening larger series, whereas, for typing of single or few samples the cost-efficient PCR-SSP method could be favored. The supply of Vel- patients with Vel- blood is required to avoid severe hemolytic reactions and is an important contribution to safer transfusion.

\section{IMH-V08}

\section{Screening for Vel negative donors: The molecular approach may be superior to serologic screening}

\section{${ }^{*}$ F. Wagner ${ }^{1}$, R. Bittner ${ }^{1}$, A. Döscher ${ }^{2}$, T. H. Müller ${ }^{1}$ \\ ${ }^{1}$ DRK Blutspendedienst NSTOB, Springe, Germany \\ ${ }^{2}$ DRK Blutspendedienst NSTOB, Institut Bremen-Oldenburg, Oldenburg, Germany}

Background: Transfusion support for patients with anti-Vel is difficult, because blood donors known to be Vel negative are rare. Last year, we started screening for Vel-negative donors using plasma from a donor with anti-Vel. Recently, the molecular basis of the Vel antigen was clarified, allowing for a molecular screening approach. We analyzed samples found by the serologic screen for the molecular Vel status.

Methods: Blood donors were tested with a diluted donor serum in Capture technique using the Galileo (Immucor). Cells from donors with a negative result were re-checked in ID gel technique with undiluted serum. A Vel PCR was devised using a HEX-labeled primer detecting the 17bp deletion present in alleles not expressing the Vel antigen, a FAM-labeled primer detecting the absence of this deletion and unlabeled non-specific reverse primers. A set of 8 different reverse primers yielding products of slightly different length was used to allow pooled analysis by capillary electrophoresis.

Results: Among 2537 donors tested on Galileo, 42 were negative and 34 doubtful for antigen Vel. Retesting in gel technique confirmed a Vel negative result for 16 of the 42 donors testing negative on Galileo and for 4 of the 34 donors with doubtful result on Galileo. Suspiciously, a crossmatch of one unit characterized as Vel negative by this strategy with a patient with a strong anti-Vel was positive. Fourteen of the 20 possibly Vel negative donors were tested by Vel PCR, only one donor was homozygous for the $17 \mathrm{bp}$ deletion, 1 donor did not carry this deletion, and 12 were heterozygous for the deletion. Both donors weakly positive in ID gel were heterozygous for the $17 \mathrm{bp}$ deletion.

Conclusion: Serologic screening for Vel negative donors is severely limited by the often low quality of the available antisera and may result in a high number of false negatives caused by Vel heterozygotes. The Vel PCR allows confirmation of the Vel negative status independent of the availability of suitable antisera. Considering the unsatisfactory specificity of serologic screening, direct molecular testing may be advantageous. The developed PCR approach allows screening of blood donors for Vel negative status by pooled capillary electrophoresis and can be integrated into our routine high throughput molecular donor blood group prediction system.

\section{IMH-V09}

CTL-2 is a new von Willebrand receptor: Role in antibody mediated neutrophil aggregation induced by anti-CTL-2 antibodies

\section{Y. Tjahyono ${ }^{1}$, B. Bayat ${ }^{1}$, H. Berghöfer ${ }^{1}$, S. Werth ${ }^{1}$, M. Burg- Roderfeld ${ }^{1}$, H. Deckmyn ${ }^{2}$, U. Sachs ${ }^{1}$, *S. Santoso ${ }^{1}$ \\ ${ }^{1}$ Justus Liebig Universität Giessen, Klinische Immunologie und Transfusionsmedizin, Giessen, Germany \\ ${ }^{2}$ Katholieke Universiteit Leuven, Laboratory for Thrombosis Research, Kortrijk, Belgium}

Background: Recruitment of neutrophils to endothelial cells lining the blood vessels is the key stage in inflammation. Recent evidence indicated that antibodies against neutrophils or/and endothelial cell could contrib- ute as active players in neutrophil recruitment (Williams et al, 2011).It is known that antibodies against choline transporter like protein-2 (CTL-2) are capable to induce stable neutrophil aggregation in vitro; a phenomenon which is associated with severe Transfusion Related Acute Lung Injury. To date, little is known about the function of CTL-2.

Methods: In this study, we sought to analyse the mechanism of neutrophil aggregation mediated by anti-CTL-2 antibodies.

Purified IgG fractions containing anti-CTL-2 antibodies were tested in granulocyte agglutination test (GAT) in the absence or presence of different purified plasma proteins (albumin, vWF, fibrinogen). ROS production was measured by the use of ferricytochrome-c reduction method. Stable transfected cells expressing CTL-2 and CD11b/CD18 were established and their interaction with purified plasma proteins was evaluated by adhesion assay and surface plasmon resonance (SPR) technology.

Results: Our results showed that neutrophil aggregation mediated by anti-CTL-2 antibodies depends on vWF. In the presence of recombinant vWF-A1-A2-A3, vWF-A1 but not vWF-A3 domain, neutrophil aggregates could be observed. This aggregation could be inhibited by mab against vWF-A1 domain and against CD11b/CD18. Interestingly, neutrophils incubated with anti-CTL-2 antibodies in the presence of soluble vWF did not lead to ROS production. However, significant ROS production was detected when neutrophils interact with immobilized vWF. In cell adhesion assay, CTL-2 transfected HEK cells adhere strongly onto immobilized vWF, but not on fibrinogen. The interaction between CTL2 and vWF was significantly stronger when compared to the adhesion capability of CD11b/CD18 transfected cells on vWF. These results could be confirmed by real time protein-protein interaction analysis by SPR; CTL-2 bounds more efficiently to vWF when compared to CD11b/CD18. Conclusion: Altogether, our findings indicated the important role of vWF for anti-CTL-2 mediated neutrophil aggregation. Furthermore, this study introduced CTL-2 as new binding partner for vWF (via A1-Domain). Together with CD11b/CD18 integrin, this molecular cross talk may open new insights on the mechanism of neutrophil recruitment during inflammation

\section{IMH-V10 \\ A novel monoclonal antibody against GPIbalpha (Gi10) inhibits human platelet clearance induced by autoantibodies against GPIb/IX from ITP patients in a NOD/SCID mice model}

\section{U. Sachs', T. Bakchoul', A. Giptner', *S. Santoso'}

${ }^{1}$ Justus Liebig Universität Giessen, Klinische Immunologie und Transfusionsmedizin, Giessen, Germany

2Universität Greifswald, Immunologie und Transfusionsmedizin, Greifswald, Germany

Background: Idiopathic thrombocytopenic purpura (ITP) is an autoimmune disease caused by autoantibodies (aabs) against platelet glycoproteins (GPs) IIb/IIIa and Ib/IX. Recent evidence indicates that aabs against GPIb/IX mediate platelet destruction by adifferent mechanism in comparison to aabs against GPIIb/IIIa (Fc-independent vs. Fc-dependent pathway) (Webster et al, 2006). Accordingly, patients with ITP mediated by anti-GPIb/IX aabs seem to be less responsive to IVIG treatment ( $\mathrm{Li}$ et al, 2012). Thus, alternative therapy option should be considered for these refractory patients.

Methods: In this study, we aimed to analyse the capability of a novel mouse monoclonal antibody (mab) specific for GPIb (mab Gi10) to interfere with the binding of aabs against GPIb/IX in vitro. In addition, the capability of this mab to inhibit platelet clearance in vivo was investigated in a previously established NOD/SCID mouse model (Newman et al, 2007). Only sera from well-characterized ITP patients containing GPIb/IX aabs $(n=10)$ were included. All sera were tested by the glycoprotein specific immunoassay, MAIPA, using a panel of mabs against different GPIb/IX subunits (Ibalpha, Ibbeta and IX). The inhibitory capacity of mab Gi10 toward anti-GPIb/IX aabs was investigated by flow cytometry. Analysis of human platelet clearance by anti-GPIb/IX aabs and mab Gi10 was performed in the NOD/SCID mouse model. 
Results: Mab Gi10 reacted specifically with GPIbalpha remnant on platelets did not affect platelet function in vitro (Kiefel et al, 1991). Analysis of aabs against GPIb/IX in the MAIPA assay using mab Gi10 as capture antibody showed significant reduction of aabs reactivity when compared to other capture mabs against GPIbalpha (clone AP1, SZ2), GPIX (clone FMC25) and GPIbbeta (clone Gi22), indicating binding competition between mab Gi10 and GPIb/IX aabs. These results could be confirmed by flow cytometric analysis; pre-incubation of platelets with mab Gi10 blocked the binding of GPIb/IX aabs. When purified IgG fractions of GPIb/IX aabs or mab Gi10 were injected into the NOD/SCID mouse, clearance of human platelets from mice blood circulation were observed. However, a significant reduction of platelet clearance was detected when mice were pre-treated with deglycosylated mab Gi10 (NGM-Gi10) prior to injection of aabs against GPIb/IX .

Conclusion: These data demonstrate the capability of mab Gi10 to hinder the binding of GPIb/IX aabs and thereby to inhibit platelet clearance in vivo. The use of modified mab Gi10 (such as NGM-Gi10) may represent an alternative therapy option for selected ITP patients.

\section{IMH-V11}

\section{Methodological and clinical aspects of alloimmunization after granulocyte transfusion in patients undergoing allogeneic stem cell transplantation}

\section{*J. Flaxa', A. Rosner', K. Hölig' ${ }^{1}$, M. Bornhäuser², R. Wassmuth ${ }^{2,3}$} ${ }^{1}$ Universitätsklinikum Dresden, Transfusionsmedizin, Dresden, Germany 2Uniklinikum Dresden, Medizinische Klinik und Poliklinik I, Dresden, Germany ${ }^{3}$ Universitätsklinikum Düsseldorf, Düsseldorf, Germany

Background: In allogeneic hematopoietic stem cell transplantation (HSCT), granulocyte transfusions (GT) may be required in immunocompromised, neutropenic patients. In this context, alloimmunization against alloantigens may occur and affect HSCT outcome.

Methods: Anti-HLA and -MICA antibody response after the administration of GT in 29 patients undergoing allogeneic HSCT $(n=27)$ encompassing 109 sera was investigated before and up to six month after HSCT. Microparticle-based multiple analyte profiling assays (MMA) were used for antibody screening and specification as implemented in LabScreenMixed (LSM) and LabScreenPRA (LSP) (One Lambda, Canoga Park, CA, USA) Antigen specific antibody binding was detected by subsequent flow cytometric analysis using LabScan 100 flow cytometer (Luminex Inc., Austin, TX, USA). For data acquisition, Luminex software version 1.2.1 was used. Results: Anti-HLA class I and II antibodies emerged de-novo in eleven $(38 \%)$ and four (14\%) patients, respectively. Similarly, preformed antibodies were observed in four cases (14\%) for anti HLA class I and also four patients for anti-HLA class II antibodies. Anti-MICA antibodies were observed in eight granulocyte recipients of which three patients developed anti-MICA antibodies after GT while preformed antibodies were seen in five patients. The conversion to positivity for any of the investigated antibodies did not significantly affect overall survival or the incidence of GVHD.

Conclusion: GT-associated alloantibody conversion observed did not significantly correlate with outcome. Thus, surveillance of anti HLA antibodies in the course of GT in the context of HSCT may not be required routinely. The role of MICA antibodies in HSCT and GT, however, requires further study.

\section{IMH-V12}

\section{Granulocyte reactive antibodies and their association with red blood cell alloimmunisation}

\section{*M. Schönbacher, M. Heinzl, G. Körmöczi \\ Medizinische Universität Wien, Blutgruppenserologie und Transfusionsmedizin, Wien, Austria}

Background: The implementation of granulocyte immunological methods at the Medical University of Vienna facilitates the diagnosis of trans- fusion related acute lung injury (TRALI) in Austria. The presence of granulocyte reactive antibodies seems widespread and risk groups have still to be defined. In this study, we determined the frequency of granulocyte reactive antibodies associated with red blood cell (RBC) alloimmunisation.

Methods: 333 samples from previously pregnant women were screened for granulocyte reactive antibodies by aggregation and immunofluorescence test. 143 of these samples contained irregular anti-RBC antibodies, $190 \mathrm{did}$ not. Both groups were compared regarding the frequency of granulocyte reactive antibodies.

Results: 47 of 143 RBC alloantibody positive blood samples contained granulocyte reactive antibodies, as well as 32 of $190 \mathrm{RBC}$ alloantibody negative blood samples. Thus, significantly more samples from RBC alloimmunised women contained granulocyte reactive antibodies (32.9\%), but also a substantial number of non-RBC alloimmunised women $(16.8 \%$, $\mathrm{p}=0.001)$. The antibody prevalence increased with the number of pregnancies (15.2\% after one, $22.8 \%$ after two, $20.4 \%$ after three, $32.1 \%$ after four or more pregnancies).

Conclusion: It is a fact that pregnancy is a risk factor for the presence of granulocyte reactive antibodies. This study shows that RBC alloimmunisation represents an additional risk factor and should be considered in future pretransfusion testing.

\section{Poster: Immunhämatologie}

\section{IMH-P01}

\section{Positive outcome of transplantation with an antigen $M$-positive kidney graft in a patient with an anti-M alloantibody}

\section{${ }^{*}$ P. Yilmaz, J. Kößler, E. Weinig, A. Kobsar, E. Klinker, M. Böck}

University of Würzburg, Institute of Transfusion Medicine and Haemotherapy, Würzburg, Germany

Background: We report on a 26-year-old male patient with congenital kidney dysplasia and terminal renal failure who had developed an irregular alloantibody against the antigen $\mathrm{M}$ due to repeated blood transfusions in the past (first detection in 06/2000). The patient required dialysis since 2007. In October 2012, a postmortal antigen M- positive kidney was provided by Eurotransplant. Since the M antigen is known to be located on red cells and on epithelial cells of the kidney, the alloantibody might have influence on allograft function after transplantation.

Methods: Blood groups of donor and recipient were determined with column agglutination technology. MN antigen structures were identified serologically and with PCR. The antibody screening tests and alloantibody levels were measured repeatedly with the indirect antiglobulin test (IAT) and in sodium chloride medium (column agglutination technology). Results: Donor and recipient were identical concerning the AB0 blood groups (blood group A), but showed differences in the MN blood group systems (donor: $\mathrm{M}+\mathrm{N}+$; recipient:M-N+). Before transplantation, the irregular alloantibody $\mathrm{M}$ was detected weakly positive in the recipient's blood with a titer level of 1:1 in indirect antiglobulin test (IgG) and weakly positive in sodium chloride medium. During the follow up for two weeks, the alloantibody was no longer detectable. Creatinine levels decreased from 5.43 to $0.96 \mathrm{mg} / \mathrm{dL}$ and urea levels from 46.7 to $23.0 \mathrm{mg} /$ $\mathrm{dL}$. The glomerular filtration rate increased significantly from 14 to 101 $\mathrm{mL} / \mathrm{min} / 1.73 \mathrm{~m}^{2}$ (MDRD). Urine secretion improved daily and reached about $3000 \mathrm{~mL}$ per day when the patient was discharged two weeks after transplantation. No further dialysis was needed. The same clinical results were obtained after three months of follow-up.

Conclusion: Our case of successful kidney transplantation illustrates that the presence of pre-existing anti-M antibodies in kidney recipients does not obligatorily have a negative impact on an antigen M-positive allograft survival. Moreover, we couldn't observe any boostering effect on the 
antibody level. In contrast, the disappearance of the antibody might be caused by adsorption on M- antigen positive epithelial cells and supported by the immunosuppressive therapy (tacrolimus, mycophenolate) after transplantation.

\section{IMH-P02 \\ Blood Group Genotyping by Means of MALDI-TOF MS - first Experiences}

${ }^{*}$ B. Just ${ }^{1}$, B. Stierand-Peterwitz ${ }^{1}$, G. Schleif', C. Vollmert ${ }^{2}$, R. Deitenbeck ${ }^{1}$

${ }^{1}$ DRK-Blutspendendedienst West, Hagen, Germany

2Sequenom $\mathrm{GmbH}$, Hamburg, Germany

Background: Antibodies against high frequent antigens often lead to critical supply situations due to the inadequate availability of antigen-negative preparations. Besides routine serological testing more and more screening programs are applied by means of molecular genetic inhouse procedures or commercial test systems. We are describing our first experiences with MALDI-TOF MS. The examination of each donor was aimed to be limited to a single multiplex approach and to consider as many polymorphisms as possible, especially the known relevant alleles (Kp(ab), Lu(ab), Yt(ab), Co(ab), Fy(ab), Jk(ab), S, s).

Methods: The validation was intensively monitored by the provider Sequenom. After its completion, repeated donors of blood group 0 (rarely A) CCD.ee, ccD.EE as well as ccddee and $\mathrm{K}$ neg. should be tested [without preliminary investigation of $\mathrm{Lu}(\mathrm{b}), \mathrm{Kp}(\mathrm{b}), \mathrm{Co}(\mathrm{a})$ und $\mathrm{Yt}(\mathrm{a})]$. Negative results were to be confirmed by a CE-marked PCR-test supplemented by a serological control of a subsequent donation. Genotyping results of $\mathrm{Lu}(\mathrm{a})$, $\mathrm{Kp}(\mathrm{a}), \mathrm{Fy}(\mathrm{ab}), \mathrm{Jk}(\mathrm{ab}), \mathrm{S}$ and $\mathrm{s}$ were to be compared with previous serological results.

Results: Positive and negative results (genetically as well as serologically pre-tested samples) were confirmed in the course of validation. In addition, duplicate or multiple replicate determinations of numerous samples were carried out (4064 evaluable allele determinations showed a concordance of $99.4 \%$ ). Among 2555 donors a total of 15 "rare donors" could be detected [negative for $\mathrm{Co}(\mathrm{a})(\mathrm{n}=7), \mathrm{Yt}(\mathrm{a})(\mathrm{n}=4), \mathrm{Lu}(\mathrm{b})(\mathrm{n}=4)]$ which could be confirmed by CE-marked SSP-kits. In the meantime 7 out of 15 cases were also serologically confirmed. 1423 serological predestinations of $\mathrm{Lu}(\mathrm{a}), \mathrm{Kp}(\mathrm{a}), \mathrm{Fy}(\mathrm{ab}), \mathrm{Jk}(\mathrm{ab}), \mathrm{S}$ or s resulted in a phenotype-genotype concordance of $99.6 \%$ (5 Fy(a-b-) were not considered). 3 of 5 discrepant results were confirmed by means of a commercial PCR-test kit (2 were not tested until now). In one case a FY*X could be found which would explain the Fy(b-) phenotype. Repeated phenotyping of all 5 samples is pending (all 5 were pre-tested only once).

Conclusion: MALDI-TOF MS is suitable for high throughput genotyping of blood groups. Our investigation has shown that results correspond well with other genotyping procedures as well as phenotyping. An extended typing of blood donors would further improve the supply of patients with antibodies against the above mentioned high frequent antigens (in future supplemented by the blood group Vel). Furthermore, it has been shown that in case of antibody mixtures an improvement could also be achieved

\section{IMH-P03}

\section{Anti-D Immunization by a Serological D-negative Red Cell Concentrate}

\section{${ }^{*}$ B. Just, B. Stierand-Peterwitz, V. Schottstedt, R. Deitenbeck}

DRK-Blutspendendedienst West, Hagen, Germany

Background: Within one week a total of 14 units of blood group $0 \mathrm{RhD}$ neg. were transfused to a female patient. With one exception (Ccddee) all donors were known as ccddee, one red cell concentrate (RCC) was K pos. Prior to transfusion, the patient was tested $0 \mathrm{Rh}$ neg. (ccddee) $\mathrm{K}$ neg. 10 days after the first transfusion the antibody screen became positive (detection of an anti-D). An immunization by serological D-negative RCC
(DEL-types) is probably rare, but published subsequently. Therefore all donors involved were to be given a further check-up.

Methods: The antibody screen as well as its identification on the patient was performed by using the ID-card system (BioRad). Phenotyping of $\mathrm{RhD}$ of the donor was carried out by using partial D panel (Diagast, BioRad) of the ID-card technique. The genotype was specified by means of SSP-PCR (BAG, inno-train).

Results: In the IAT anti-D could be proven beyond doubt. 12 out of 14 donors could be re-examined so far. 11 out of 12 donors reacted negatively in the D-screen, in the SSR-PCR also no evidence was found on a partial D whereas in 1 of these 12 donors the hybrid RHD (1-9)-CE(10)/RHD(delEx10) could be suspected. Serologically this donor reacted very weak only with the monoclonal antibody P3X249. A reaction with the monoclonal anti-D HM16 is reported which was negative in our investigations. Therefore it cannot be excluded that only sequencing admits an unambiguous determination of the allele. On the occasion of a further donation additional examinations (like absorption/elution, sequencing) are planned.

Conclusion: Our findings support an anti-D immunization by a DEL serotyped as Ccddee. RHD (1-9)-CE(10)/RHD(delEx10) is ascribed a low density of 50 antigens per red cell. Blood products from a donor with an obvious weakened D-expression indicate a certain immunization potential; this is why some blood donation services carry out the genotyping of serologically D-negative donors. Determination of DEL can hardly/ cannot be achieved by serological routine methods with regard to DELtypes with only few D-epitopes per red cell. Complementary techniques should be routinely added.

\section{IMH-P04 \\ HLA Class II Haplotypes in Autoimmune Thyroid and in Polyglandular Disease}

${ }^{\star}$ B. Flesch ${ }^{1}$, T. Alt ${ }^{1}$, N. Matheis' ${ }^{2}$, C. Weinstock ${ }^{3,4}$, J. Bux ${ }^{1}$, G. Kahaly ${ }^{2}$

1DRK Blutspendedienst West, Hagen und Bad Kreuznach, Germany 2Universitätsklinikum, Mainz, Germany

${ }^{3}$ DRK Blutspendedienst West, Bad Kreuznach, Germany

${ }^{4}$ DRK Blutspendedienst Baden-Württemberg-Hessen, Ulm, Germany

Background: The association of autoimmune thyroid diseases (AITD) with HLA class II alleles and haplotypes has been demonstrated before. In contrast, immunogenetics of the autoimmune polyglandular syndrome type III (APS) involving both, type 1 diabetes and AITD, is poorly understood. Therefore, we compared the HLA class II alleles, haplotypes and genotypes in a large collective of patients with AITD, APS and euthyroid healthy controls.

Methods: A total of 222 patients with AITD, 93 with APS and 121 non-related healthy subjects were typed for $D R B 1, D Q A 1$ and $D Q B 1$ at a four digit level. For high resolution HLA typing a bead-based PCR-SSO technique (Luminex ${ }^{\circledR}$ ), PCR with sequence specific primers (PCR-SSP), or an in-house PCR-method with sequence based typing (PCR-SBT) were used. HLA DRB1-DQA1-DQB1 haplotypes were deduced either from family based segregation, where family members were available, or from the known allele linkage.

Results: The allele and haplotype frequencies significantly discriminated patients with AITD versus APS. The alleles DRB1*03:01, *04:01, $D Q A 1 * 03: 01, * 05: 01$ and $D Q B 1 * 02: 01, * 03: 02$ were more prevalent $(P<0.001)$ in APS than in AITD patients and controls. In contrast, $D R B 1 * 11: 01, * 15: 01, D Q B 1 * 03: 01$ and $* 06: 02$ predominated in AITD. The haplotypes $D R B 1 * 03: 01-D Q A 1 * 05: 01-D Q B 1 * 02: 01$ and $D R B 1 * 04: 01-D Q A 1 * 03: 01-D Q B 1 * 03: 02$ were strongly overrepresented in APS versus both AITD $\left(P_{c}<0.001\right)$ and controls $\left(P_{c}<0.001\right.$; fig. 1$)$. The combination of both haplotypes to a genotype was also highly prevalent in APS patients versus both controls and AITD patients (OR 28.8 and $26.4, P<0.001$; fig. 2). Finally, the haplotypes $D R B 1 * 11: 01-D Q A$ $1 * 05: 05-D Q B 1 * 03: 01$ and $D R B 1 * 15: 01-D Q A 1 * 01: 02-D Q B 1 * 06: 02$ were protective for APS compared to controls $\left(P_{c}<0.01\right.$ both) and to $\operatorname{AITD}\left(P_{c}<0.01, P_{c}<0.001\right)$. 
Conclusion: HLA class II haplotypes and genotypes differentiate between AITD and APS. Susceptibility haplotypes favor the development of polyglandular autoimmunity in AITD patients. Especially AITD patients with the $D Q A 1 * 05: 01-D Q B 1 * 02: 01 / D R B 1 * 04: 01-D Q A 1 * 03: 01$ $D Q B 1^{*}$ 03:02 genotype carry an increased risk to develop an additional autoimmune glandular disease.

Fig. 1

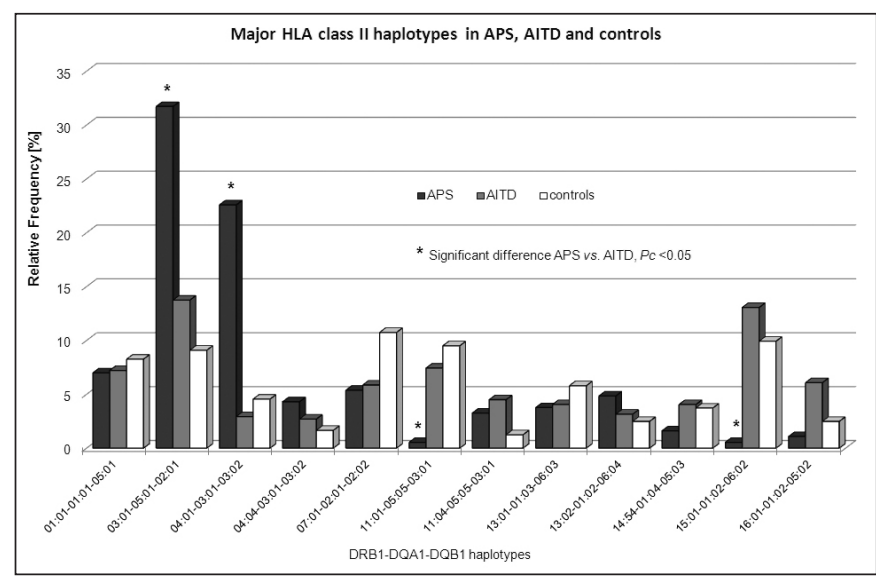

Fig. 2

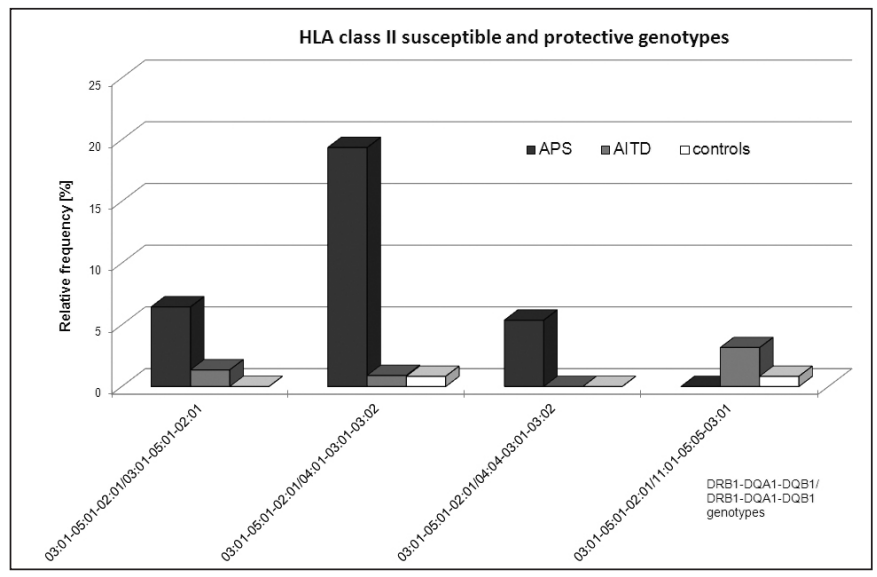

IMH-P05

\section{Mixed field agglutination in weak $D$ type 31 : No Rh complex alteration except for diminished Band3}

\section{J. Reif', H. Hustinx², E.-M. Dauber ${ }^{1},{ }^{*}$ G. Körmöczi ${ }^{1}$}

${ }^{1}$ Medical University of Vienna, Blood Group Serology and Transfusion Medicine, Vienna, Austria

${ }^{2}$ Regional Blood Transfusion Service of the Swiss Red Cross, Bern, Switzerland

Background: Some weak D type 31 variant red blood cell (RBC) samples exhibited spontaneous mixed field agglutination in routine serological RhD typing. No definitive cause of the observed mixed field agglutination could be determined, as established reasons of this phenomenon could be excluded in all cases. Therefore, these samples were investigated for alterations of Rh complex composition potentially leading to mixed D expression.

Methods: Flow cytometric D antigen density measurement and Rh complex analysis was applied to five weak D type 31 samples with spontaneous mixed field agglutination, and compared to weak D variants without mixed field agglutination as well as Rh phenotype-matched controls. Results: The serological RhD mixed field pattern of the weak D type 31 samples was confirmed by flow cytometry. With the standard algorithm, the median D antigen density of weak D type 31 RBCs was 176 (range 117-230). Using an alternative calculation method, the D antigen density was determined separately for each RBC subpopulation: one RBC population with distinctly lower (median 72; range 46-124) and another population with markedly higher D density (median 1855; range 1572-2389). To evaluate for a potential alteration of the Rh complex, the expression of $\mathrm{C}, \mathrm{c}, \mathrm{E}$, and e antigens, total Rh, Rh-associated glycoprotein, Band3, CD47 and LW protein were quantified. A significantly reduced Band3 expression on the weak D type 31 variants was observed, whereas expression of all other Rh complex molecules showed no abnormalities, compared to controls.

Conclusion: Band 3 is known to be an important factor for correct plasma membrane integration of the RhD polypeptide. Therefore, Band 3 expression irregularities may contribute to the mixed D expression of the studied weak D type 31 samples. So far, however, the mechanism for altered Band 3 expression in these samples remains unknown.

\section{IMH-P06 \\ Flow-cytometric detection of leukocyte-reactive antibodies: Partial association with red cell alloimmunization}

\section{Heinzl, M. Schönbacher, *G. Körmöczi}

Medical University of Vienna, Blood Group Serology and Transfusion Medicine, Vienna, Austria

Background: Anti-granulocyte antibodies are directed against human leukocyte antigens or human neutrophil antigens (HNA) and may cause transfusion-related acute lung injury and immune neutropenias. The flow cytometric granulocyte immunofluorescence test (Flow-GIFT) may facilitate large-scale screening for anti-leukocyte antibodies. Particular risk groups of anti-HNA immunization are yet to be defined. In this study, a possible association of white and red blood cell (RBC) alloimmunization was determined using Flow-GIFT.

Methods: Of 333 samples from women with a history of pregnancy, 143 contained irregular anti-RBC antibodies and 190 did not. These groups were tested by Flow-GIFT and compared for the frequency of anti-leukocyte antibodies.

Results: The frequency of granulocyte reactive antibodies was $38.5 \%$ (55 of 143 samples) in the anti-RBC alloimmunized group and $29.5 \%$ (56 of 190 samples) in the control group $(p=0.085)$. Anti-RBC alloimmunization was thus not a risk factor for anti-granulocyte reactivity. The difference regarding anti-lymphocyte reactivity was significant $(\mathrm{p}=0.035)$ when not adjusted for the number of pregnancies. Twelve positive control samples were granulocyte-reactive in Flow-GIFT and specificity proved to be $95.5 \%$ when testing 22 negative controls. Importantly, characteristic differences between serum and plasma samples were evident when performing Flow-GIFT, with impact on fluorescence values.

Conclusion: This study did not show an association between anti-RBC and anti-granulocyte alloimmunization, but possible associations may have been blurred by technical effects (serum/plasma). However, anti-lymphocyte antibodies seem to be associated with anti-RBC alloimmunization. Flow-GIFT may be the ideal method for large-scale anti-granulocyte antibody screening due to its high sensitivity. It is recommended to analyze serum samples. 


\section{IMH-P07}

\section{Alloantibody development in a patient with the Rhesus blood group D-}

${ }^{\star}$ E. Weinig' ${ }^{1}$ J. Kößler ${ }^{1}$, P. Yilmaz' 1 , E. Scharberg' ${ }^{2}$ F. Wagner ${ }^{3}$, A. Döscher ${ }^{4}$, E. Klinker ${ }^{1}$, M. Böck ${ }^{1}$

${ }^{1}$ University of Würzburg, Institute of Transfusion Medicine and Haemotherapy, Würzburg, Germany

${ }^{2}$ Red Cross Blood Service Baden-Württemberg-Hessen, Institute BadenBaden, Baden-Baden, Germany

${ }^{3}$ Red Cross Blood Service NSTOB, Institute Springe, Springe, Germany

${ }^{4}$ Red Cross Blood Service NSTOB, Institute Bremen-Oldenburg,

Oldenburg, Germany

Background: We report on a male 45-year-old patient with the rare Rhesus phenotype D-. The patient suffered from M. Castleman (generalized lymphadenopathy) and was currently treated in our hospital preparing peritoneal dialysis because of acute renal failure. 24 days before admission, he received one standard unit of packed red cells $0 \mathrm{D}+(\mathrm{CcD}$.ee $)$ in an external hospital - incompatible concerning the Rhesus phenotype. We followed up the patient in respect to antibody development and clinical course.

Methods: Blood group determination, antibody screening and antibody identification tests were performed using column agglutination technique (DiaMed Diagnostica, Munich, Germany), antibody identification in addition with tube technique (Grifols Diagnostics, Düdingen, Switzerland). $\mathrm{ABO}$ and Rhesus CDE blood group antigens were confirmed by PCR (Inno-Train Diagnostik, Kronberg, Germany). Sequencing of the Rhesus gene RHCE, Exon 1-9 (patient and his sister) was performed using appropriate primers.

Results: 27 days after transfusion, the patient had developed an alloantibody with an apparent preference for Rhesus e only in the enzyme medium. In the course of follow-up, singular reactions appeared also in the indirect antiglobulin test (IAT, day 31 and 43). Finally, on day 105, the patient had a strong panreactive alloantibody detectable in the IAT and the enzyme medium with preference for Rhesus $\mathrm{C}$ and $\mathrm{e}$ (in the sodium chloride medium). The serum reacted negatively only with two samples of D- (Rh17 negative) and two samples of Rhnull test cells showing a clear anti-Rh17 (anti-Hro) specificity. Sequencing of the Rhesus gene showed that the patient and his sister are homozygous for the RH allele RHCE(1)-D(2-9)-CE(10), also known as D 2-9 D-SH.

Conclusion: A patient with the genetically confirmed rare Rhesus phenotype $\mathrm{D}$ - developed a panreactive alloantibody against antigens of the $\mathrm{RhCE}$ protein after transfusion of only one unit of packed red cells. This alloantibody is known as anti-Hro or anti-Rh17. Anti-Rh17 of different patients are not necessarily mutually compatible. In consequence, all efforts have to be done to avoid incompatible transfusions. Donors with this rare $\mathrm{Rh}$ phenotype must be registered in (inter)national rare donor programs.

\section{IMH-P08}

\section{DNA-extraction from cell free maternal plasma with the SNAPcard ${ }^{\mathrm{TM}}$ method}

${ }^{*}$ A. Doescher ${ }^{1}$, F.F. Wagner ${ }^{2}$, C. Vogt ${ }^{1}$, H. Paul ${ }^{3}$, A. Ross ${ }^{3}$,

\section{E.J. Klip ${ }^{3}$, E.K. Petershofen ${ }^{1}$}

${ }^{1}$ Blutspendedienst NSTOB, Oldenburg, Germany

${ }^{2}$ Blutspendedienst NSTOB, Springe, Germany

${ }^{3}$ Blood Cell Storage Inc., Seattle, Germany

Background: Determination of HDN-relevant fetal blood groups in plasma of pregnant women is increasingly used as an additional diagnostic method. Polymorphisms are used to either discriminate between fetal and maternal DNA or to verify negative test results. Technologies for DNA extraction play an important role in this aspect and different technologies for plasma extraction are available. DNA extraction from cell free plasma by binding to the surface of a glass slide is a new approach is this context We compared this new method with our routinely performed magnetic particle procedure
Methods: DNA from maternal plasma samples (at gestation week 15th to 18th) was extracted in parallel with the MagnaPure ${ }^{\mathrm{TM}}$ large volume DNA isolation kit and the BCSI SNAPcard ${ }^{\mathrm{TM}}$ with a sample volume of $500 \mu \mathrm{L}$. DNA yield was examined by UV-spectroscopy. In addition, the amount of fetal DNA was examined by quantitative real-time PCR. Amplification of $R H D$ specific sequences and internal controls were performed with TaqMan PCR and single base extension method.

Results: Samples from 50 pregnant women were tested in parallel. The mean yield for SNAPcard DNA extraction was $45 \mathrm{ng} / \mu \mathrm{L}$ (range 22-91) compared to $18 \mathrm{ng} / \mu \mathrm{L}$ (range 5-27) using magnetic particles. No differences could be observed for threshold cycles in real-time amplification of RHD Exon 10 comparing samples extracted with either method. Single base extension assays for determination of gender-independent internal controls showed more and/or higher peaks for paternal single nucleotide polymorphisms in about $70 \%$ of the SNAPcard extracted samples.

Conclusion: Our results demonstrate success for extraction of cell free DNA from plasma on glass slides in this setting. The SNAPcard provides a higher yield of nucleic acids and a DNA quality comparable in real-time PCR settings and better results for high multiplexed amplification assays in prenatal diagnosis.

\section{IMH-P09 \\ Four premature stop-codons in FUT 1 gene cause two different $\mathrm{H}$-deficient blood groups detected in blood donors}

\author{
${ }^{*}$ A. Doescher ${ }^{1}$, G. Fauchald², J. Lüdecke ${ }^{3}$, F.F. Wagner ${ }^{4}$ \\ ${ }^{1}$ Blutspendedienst NSTOB, Oldenburg, Germany \\ ${ }^{2}$ BRK Blutspendedienst, München, Germany \\ ${ }^{3}$ BRK Blutspendedienst, Nürnberg, Germany \\ ${ }^{4}$ Blutspendedienst NSTOB, Springe, Germany
}

Background: Polymorphisms in the FUT 1 gene affect the expression of the $\mathrm{H}$ blood group phenotype. Homozygous non-expressed alleles of FUT 1 exhibit either the Bombay phenotype, if no active Se transferase is present. Non-expressed FUT1 alleles linked to active Se transferases and weakly active FUT1 alleles may result in "para-Bombay" phenotypes with severely diminished expression of antigens A, B or H on the RBC. Here we present two cases of FUT 1 alterations in blood donors from Germany.

Methods: Serological examination of H-deficient blood groups was performed with routine methods including antigen determination with commercial antisera and ID panels. Molecular analysis was done by sequencing of exon 1 to 4 including intron/exon borders of the FUT 1 gene, using BigDye terminators v 1.1. Further examinations of the new polymorphisms were done by haplotype specific sequencing, using primers in the exon 1 insertion.

Results: A) The H-deficient A blood group from the donor from Northwest Germany initially appeared as weakly positive for antigen A but negative for antigens $\mathrm{H}$ and A1. Molecular analysis showed one allele with a seven basepair insertion in exon 1 of the FUT 1 gene (M93X). An additional substitution of $\mathrm{C}>\mathrm{T}$ at the second allele results in a premature stop at aa 104. Haplotype specific sequencing confirms the distribution of the mutations of both alleles.

B) All serological signs for a para-bombay type were observed in the sample of a blood donor. Molecular analysis reveals $\mathrm{C}>\mathrm{T}$ polymorphisms at nt 586 and 836 (stop-codons at positions 196 and 256).Examination of maternal and paternal DNA shows the heterozygous para-Bombay type for the mother and the para-Bombay type USA2 for the father. Due to the heterozygous constellation, the parents show no signs of a H-deficient blood group.

Conclusion: Serological suspect of H-deficient blood group could be confirmed by molecular genetic analysis. Each of the donors harboured two different mutations und therefore premature stop-codons at different positions. The phenotype of both donors showed a weak or absent expression of the $\mathrm{H}$ antigen. Mutations in the FUT1 gene may result in apparent weak A or B phenotypes. 


\section{IMH-P10}

\section{Anti-D antibody in a pregnancy with a deletion in exon 1 of the maternal RHD gene}

\author{
${ }^{*}$ A. Doescher ${ }^{1}$, C. Vogt ${ }^{1}$, E.K. Petershofen 1 , F.F. Wagner ${ }^{2}$ \\ ${ }^{1}$ Blutspendedienst NSTOB, Oldenburg, Germany \\ ${ }^{2}$ Blutspendedienst NSTOB, Springe, Germany
}

Background: Determination of HDN-relevant fetal blood groups in amniocentic fluid or from maternal plasma is a routinely used tool in pregnancies with known antibodies. While the detection of RHD-positive DNA in a $R H D$-negative background should be no problem, the occurrence of $R H D$-sequences in RhD-negative mothers can give rise to false positive typing.

Methods: DNA from $1 \mathrm{ml}$ plasma was extracted using the MagnaPure Large Volume protocol and tested for the presence of RHD exons 3 and 10. DNA from maternal leukocytes was isolated with the PureGeneÔ Kit RHD genotyping of maternal DNA was done using an in-house multiplex PCR setting. Sequencing of $R H D$-sequences, including exons 1 to 10 and intron/exon borders, were done by direct taq cycle-sequencing using BigDye-terminators v.1.1 in an ABI310. Determination of antigen density was performed using 12 anti-D sera (LHM 75/58, 76/59/, 174/102, 50/28, $169 / 81,76 / 55,77 / 64,70 / 45 /, 59 / 19,169 / 80,57 / 17$, ESD1). The presence of a Del phenotype was tested with absorption and elution technique using a polyclonal anti-D (BioRad).

Results: Both DNA samples (plasma and maternal DNA) showed positive reactions for $R H D$-specific exons. Sequencing of the maternal DNA for $R H D$ exons 1 to 10 reveals a deletion of nucleotide "A" at position 147 in exon 1 of the RHD gene. Mean value for the determination of antigen density was 3.0 and below the given value for the ccddee-control (3.9). In addition, no antigen $\mathrm{D}$ could be detected by absorption and elution technique in this case.

Conclusion: Prenatal determination of fetal $R H D$ is limited in cases when the mother carries a non-expressed D-allele. Since maternal plasma includes both, DNA from maternal and fetal cells, during pregnancy it is not possible to designate the origin of the PCR templates. The determination of the anti-D titer should be performed on a regular basis, e.g. two times a month. Although, the "del 147 A" allele was first mentioned in 2004 and characterized as DEL, adsorption/elution and flow cytometry suggest that in our case a D negative phenotype may be present.

\section{IMH-P11 \\ Comparison of Three Different Assays for Platelet Antibody Testing}

\author{
${ }^{*}$ N. Sareban ${ }^{1}$, S. Macher ${ }^{1}$, G. Lanzer ${ }^{1}$, C. Drexler ${ }^{1}$, U. Posch ${ }^{1}$ \\ K. Schallmoser ${ }^{2}$ \\ ${ }^{1}$ LKH Graz, Universitätsklinik für Blutgruppenserologie und \\ Transfusionsmedizin, Graz, Austria \\ ${ }^{2} \mathrm{LKH}$ Salzburg, Universitätsklinik für Blutgruppenserologie und \\ Transfusionsmedizin, Salzburg, Austria
}

Background: Platelet reactive antibodies against glycoproteins (GP), HLA class I antigens or human platelet antigens (HPA) may lead to significant thrombocytopenia and bleeding disorders in various clinical syndromes. A wide range of different assays are used for their detection in clinical laboratories. In this retrospective analysis the suitability of three different test systems was examined and the spectrum of antibody specificities was assessed.

Methods: From 2008 to 2011 serum samples of 1,250 thrombocytopenic patients with suspected autoimmune thrombocytopenia (ITP), malignant hematologic diseases, transfusion reaction to platelet concentrates, refractoriness to platelet transfusions and suspected neonatal alloimmune thrombocytopenia (NAIT) were tested for platelet antibodies with an enzyme-linked immunosorbent assay (ELISA; Lifecodes PAKPLUS ${ }^{\circledR}$ and PAK $12^{\circledR}$, Gen-Probe) and a solid-phase assay (Capture-P Ready Screen ${ }^{\circledR}$, Immucor Inc.). In cases of suspected anti-HLA class I antibodies a specific lymphocytotoxicity test (LCT, Bio-Rad ${ }^{\circledR}$ ) was additionally performed.
Results: In total, 382/1,250 samples were positive in the ELISA or in the solid-phase assay (30.6\%). In $69.4 \%$ we obtained concordant negative but only in $8.6 \%$ concordant positive results with both methods implying that 197 samples of 1,065 in the solid-phase assay negative samples were positive in the ELISA (18.5\%). The most frequent specificities of antibodies, only detectable in the ELISA were GP IIb/IIIa (36/197; 18.3\%), GP Ia/IIa $(28 / 197 ; 14.2 \%)$ and combined GP IIb/IIIa and GP Ia/IIa (45/197; $22.8 \%$ ). In the group of samples tested positive by both methods the majority of antibodies reacted against HLA class I antigens $(42 / 107 ; 39.3 \%)$ followed by GP IIb/IIIa (10/107; 9.3\%). The frequency of HPA-specific antibodies was comparable in both groups $(7 / 197 ; 3.6 \%$ and $4 / 107$; $3.7 \%$ ). All other specificities were rare.

Conclusion: In contrast to HLA-class I and HPA antibodies, GP-specific platelet antibodies were more frequently detectable in the ELISA than in the solid phase assay. Therefore, suitability of the different methods seems to depend on suspected clinical diagnoses. In our laboratory the ELISA and LCT are the first line screening tests, the solid-phase assay is used as additional test to confirm HPA antibodies. However, in cases of uncertain results analysis by the 'monoclonal antibody-specific immobilization of platelet antigen’ (MAIPA) - assay is highly recommended.

\section{IMH-P12}

\section{Case Report: Prevention of Fetal Hydrops - Non-invasive Doppler Ultrasonography, Immunohematological Diagnostics during a Risk Pregnancy}

\section{${ }^{*}$ T.J. Schulze' ${ }^{1}$, M. Goebel', K. Heuser'2, S. Götz ${ }^{3}$,} E.K. Petershofen ${ }^{4}$, G. Hütter ${ }^{1}$, K. Janetzko ${ }^{1}$

${ }^{1}$ Institute of Transfusion Medicine and Immunology, German Red Cross Blood Service, Mannheim, Germany

2Diakonissen-Stiftungskrankenhaus Speyer, Clinic for Gynaecology and Obstetrics, Speyer, Germany

${ }^{3}$ IKTZ Heidelberg gemeinnützige $\mathrm{GmbH}$, German Red Cross Blood Service, Heidelberg, Germany

${ }^{4}$ Institut Bremen-Oldenburg, German Red Cross Blood Service, Oldenburg, Germany

Background: A 30 year old woman (para 1, gravida 2) with pre-existing anti-K at a high titre and a newly diagnosed anti-Jk(a) antibody underwent gynecological and immunohematological control examinations during pregnancy. Anti-K not only causes severe HDN but also inhibits erythroid progenitors leading to prolonged anemia. NAT testing of fetal DNA was negative for Kel-1. Anti-K titres rose although clinical examination and ultrasonography excluded hemolysis. Gynecologists decided to deliver full-term.

Methods: Indirect antiglobuline test (IAT) - for both antibody detection and titre measurements - and direct antiglobuline test (DAT) were performed in the gel ID card system (DiaMed-BioRad). Elution was performed with chloroform technique. $\mathrm{K}$ and $\mathrm{Jk}(\mathrm{a})$ antigens were detected by tube test with two different clones (Medion-Optima, Immucor-Optima). DNA was examined by NAT for Kel-1 (Petershofen, Oldenburg, Germany). Fetal arterial blood flow (Vmax) of the arteria cerebri media was performed by doppler ultrasonography (Toshiba Xario). Titre elevation was determined by testing both the presently and last tested material within one set.

Results: Fetal DNA was initially tested negative for Kel-1 in week 10 . Anti-K antibody titre by then was $2^{11}$. In week 22 , anti-Jk(a) was newly diagnosed with titre $2^{1}$. Anti-K titres in week 36, 38, 39, 40, 41 were between $2^{11}$ and $2^{13}$ while anti-Jk(a) titre rose from $2^{1}$ to $2^{3}$ by week 41 . Clinical and ultrasonographical examination, especially Vmax of arteria cerebri media, performed at the same time, were all within normal range. At birth, week $41+4$, anti-K titre was $2^{14}$ and anti-Jk(a) was $2^{0}$ in the newborn's plasma. The newborn was K negative and Jk(a) positive. DAT was positive and anti-Jk(a) could be eluted from the child's red blood cells. There were no signs of icterus or hemolysis. Regular follow-up examination did not show any abnormalities. 
Conclusion: After negative molecular biological testing for Kel-1, despite high anti-K titres, gynecologists performed clinical examination together with Doppler ultrasonography and immunohematological titre controls instead of inducing delivery. Both, prenatal antigen detection and Vmax measurement of the arteria cerebri media using Doppler ultrasonography that correlate precisely to Hb-Levels in the fetal blood prove to be reliable and feasible diagnostics that can help avoid invasive procedures.

\section{IMH-P13}

\section{Case Report: Administration of incompatible red cell concentrates to patients with Anti-ABTI}

${ }^{*}$ G. Fauchald1', B. Lucke' ${ }^{1}$ H. Hannibal', J. Lüdecke', H. Butsch ${ }^{1}$, F. Weinauer ${ }^{1}$

${ }^{1}$ BSD BRK, München, Germany

${ }^{2}$ Klinikum Main-Spessart, Würzburg, Germany

Background: An 86 year old patient with known anti-ABTI required a transfusion after a femur fracture. ABTI is a highly common antigen. Side effects after a required transfusion are highly probable but as yet have not been described.

Methods: After operative treatment of the femur fracture the patient was transfused under intensive-care monitoring and appropriate clinical chemistry and immunhaematological examinations were performed. The five children of the patient were tested for ABTI and VEL-Antigen.

Results: The Hemoglobin value after transfusion was $7.0 \mathrm{~g} / \mathrm{dl}$. An anti-ABTI titre of 1:32 in albumin IAT before transfusion with DCT negative fell to 1:4 during AK adsorption with positive DCT after transfusion. 48 hours after transfusion haptoglobin was $68 \mathrm{ml} / \mathrm{dl}$, LDH was $787 \mathrm{U} / 1$ and total bilirubin was $1.55 \mathrm{mg} / \mathrm{dl}$. Using adsorption and elution techniques we were able to produce an anti-ABTI test serum that can be used for all blood groups.

Conclusion: No transfusion reaction was observed in a patient with an initial anti-ABTI titre of 1:32 after transfusion of ABTI positive blood. For clarification of suspected anti-ABTI cases we produced a test serum with which anti-ABTI can be determined.

\section{IMH-P14}

\section{High-titer anti-D in a blood donor with $R H D(I V S 3+2 T>A)$}

\section{*I. Mardt ${ }^{1}$, A. Döscher ${ }^{2}$, F. Wagner}

${ }^{1}$ DRK Blutspendedienst NSTOB, Institut Springe, Springe, Germany ${ }^{2}$ DRK Blutspendedienst NSTOB, Institut Bremen-Oldenburg, Oldenburg, Germany

Background: Splice site mutation in $R H D$ are often associated with a DEL phenotype, but D negative phenotypes may occur, too. The discrimination of DEL and D negative alleles may be difficult and variable results have been obtained e.g. for $R H D(\mathrm{IVS} 3+1 \mathrm{G}>\mathrm{A})$. $R H D(\mathrm{IVS} 3+2 \mathrm{~T}>\mathrm{A})$ has been described as $\mathrm{D}$ negative, but formal proof for anti-D production was lacking.

Methods: Blood donors antibody screen was performed using Capture technique on Galileo (Immucor). Titers were performed in ID gel technique. D negative first time donors were screened by RHD PCR for RHD exon 7 in pools of about 20; the molecular basis was determined by $R H D$ sequencing.

Results: A donor with a high-titer anti-D and RH phenotype ccddEe was found to be positive for $R H D$. Sequencing revealed the $R H D$ allele RHD(IVS3 $+2 T>A)$. The anti-D titer was 1:256. As possible immunizing event, there was a pregnancy in 2012. No transfusions had been reported. Conclusion: Individuals with $R H D(\operatorname{IVS} 3+2 \mathrm{~T}>\mathrm{A})$ are able to produce high-titer anti-D, similar to other D negative individuals.

\section{IMH-P15}

\section{New RHD alleles with weak hemagglutination and genetic exon 9 diversity: weak D Types 45.1, 75, and 76.}

\author{
${ }^{\star}$ C. Gassner ${ }^{1}$, I. Utz ${ }^{2}$, H. Schennach ${ }^{2}$, A. Ramoni ${ }^{3}, H$. Steiner ${ }^{4}$, \\ S. Scholz ${ }^{5}$, U. Kreklau ${ }^{6}$, G. Körmöczi ${ }^{7}$
}

${ }^{1}$ Blood Transfusion Service Zurich, Swiss Red Cross, Molecular

Diagnostics \& Cytometry, Schlieren, Switzerland, Switzerland

${ }^{2}$ General Hospital and Univ. Clinics, Central Institute for Blood Transfusion

and Immunological Department, Innsbruck, Austria, Palestine

${ }^{3}$ General Hospital and Univ. Clinics, Department of Obstetrics and

Gynecology, Innsbruck, Austria, Austria

${ }^{4}$ General Hospital and Univ. Clinics, Department of Urology, Innsbruck,

Austria, Austria

5 InnoTrain GmbH, Kronberg i.T., Germany, Germany

${ }^{6}$ Labor Wisplinghoff, Köln, Germany, Germany

${ }^{7}$ Medical University of Vienna, Department of Blood Group Serology and

Transfusion Medicine, Vienna, Austria, Austria

Background: Weak agglutination of $\mathrm{RhD}$ positive samples may be observed for most category and partial Ds, which are prone to anti-D development, and among weak Ds, usually tolerating confrontation with RhD upon pregnancy, or transfusion, without developing anti-D. Still, new weak D alleles are discovered on a regular basis and are then ideally described by molecular variant $R H D$ allele analysis, complemented by detailed characterization of the associated D phenotype.

Methods: Sample 45.1 originated from Labor Wisplinghoff, Cologne, Germany, and samples 75 and 76 were both patients from the General Hospital and University Clinics in Innsbruck, Austria. Variant D types were characterized using PCR-SSP based molecular typing, RHD sequencing, extended serologic D antigen investigations, and flow cytometric $\mathrm{D}$ antigen quantification.

Results: Three novel weak D types termed weak D Types 45.1, 75, and 76 with $R H D$ nucleotide substitutions coding for amino acid exchanges in predicted intracellular $\mathrm{RhD}$ polypeptide stretches were discovered by standard serological methods. Flow cytometric analysis determined their antigen densities to be approximately $1.990,900$, and $240 \mathrm{D}$ sites per red blood cell, respectively. Adsorption-elution-technique-supported D epitope mapping of these three weak D types demonstrated the expression of all tested D epitopes. All three novel weak D Types 45.1, 75, and 76 alleles share a remarkable common feature, in that all of them typed negative for coding nucleotide A1193 located in Exon 9 of the RHD gene. This could clearly be shown by $R H D$ gene exon scanning polymerase chain reaction using sequence-specific priming and was explainable by specific mutations for weak D Types 45.1 (C818T, G1195A), 75 (G1194C), and 76 (A1215C).

Conclusion: All novel weak D types expressed all tested D epitopes. Therefore, in respective carriers, immunization-tendency upon challenge may be small, but can't be excluded in general. It is of interest that for weak D Types 45.1, 75, and 76, similar alleles with a maximal divergence of one amino acid only, that is, weak D Types 45,41 , and 68 , respectively, have been reported so far.

\section{IMH-P16}

\section{Clinical application of non-invasive fetal blood group genotyping in Upper Austria}

\section{${ }^{*}$ H. Polin 1 , A. Reiter ${ }^{1}$, M. Brisner ${ }^{1}$, M. Danzer ${ }^{2}$, J. Weinberger ${ }^{2}$, C. Gabrie/ $^{3}$}

${ }^{1}$ Red Cross Transfusion Service of Upper Austria, Molecular Immunohematology, Linz, Austria

${ }^{2}$ Red Cross Transfusion Service of Upper Austria, Immunogenetics, Linz, Austria

${ }^{3}$ Red Cross Transfusion Service of Upper Austria, Head of Departement, Linz, Austria

Background: In the past decade, non-invasive prenatal diagnosis (NIPD) of cell-free fetal (cff) DNA has been implemented in many routine laboratories across Europe. Diagnostic approaches encompass the detection 
and characterization of blood group markers, hemoglobinopathies and chromosomal aberrations. Clinically employed assays require reliable detection of cff DNA for the exclusion of false-negative results. In 2005, NIPD for RHD, RHC, RHE and KEL1 specific nucleotide alterations was introduced in our institute. In a look-back, we evaluated the feasibility of our genotyping strategy.

Methods: Cell-free DNA was isolated from a total of 122 maternal plasma samples from gestation weeks 9-40. Real-time PCR for RHD exon 7 and $10(\mathrm{n}=88)$, RHC $(\mathrm{n}=16)$, RHE $(\mathrm{n}=13)$ or KEL1 $(\mathrm{n}=5)$ was conducted in parallel to an Internal Control fragment (b-globin). In case of negative reactions, the presence of cff DNA was confirmed by 1 to 9 follow-up PCRs based on genetic conditions inherited from the father. All results were compared with postnatal serology.

Results: In total, we observed two (1.6\%) inconclusive results during this retrospective study. RHD exon 7 failed to amplify in one sample at gestation week 9. However, PCR testing three weeks later revealed positive results. One sample did not meet the threshold criteria due to non-amplification of the Internal Control segment, probably based on insufficient DNA extraction. There was no evidence for variant alleles. When compared to postnatal results, $100 \%$ concordance was observed.

Paternally derived single nucleotide polymorphisms (SNPs) were confirmed in 14 samples without blood-group specific markers. Y-chromosomal markers were amplified in 9 samples determined by a single PCR. In 5 samples, additional PCRs were carried out to detect the presence of fetal DNA.

Conclusion: Our real-time based approach for non-invasive characterization of fetal blood group markers was proven rapid and specific. The reliability of the methodology is provided post gestation week 12 . The presence of paternal mutations in the fetal genome was reliably identified at various periods of gestation.

\section{IMH-P17}

\section{An extended plasma inhibition test to discriminate Anti- Ch1 and Anti-Rg1}

\section{${ }^{*}$ A. Stenzel, G. Bringmann, C. Baumann, T. Zeiler}

DRK-Blutspendedienst West, Immunhämatologie, Ratingen, Germany

Background: Anti-Ch1 (Chido) and Anti-Rg1 (Rodgers) antibodies are clinically insignificant but are able to disturb immunohematologic analyses due to their reactivity in the indirect antiglobuline test (IAT).

Anti-Ch1 and Anti-Rg1 can be inhibited by normal plasma (inhibition test), however further investigations are necessary to determine the exact specificity.

Here we present a selective inhibition test using plasma from $\mathrm{Ch} 1$ and Rg1 negative donors.

Methods: We tested blood donors for the presence of HTLA-antigens (Ch1, Rg1, Kn(a), Kn(b), McC(a), McC(b), Yk(a+), Yk(a-)) via in-house PCR and identified Ch1- and Rg1-negative donors.

Extended Inhibition Test:

$100 \mu 1$ plasma from patients with suspected Anti-Ch1 or Anti-Rg1 are diluted with $100 \mu \mathrm{l}$ normal plasma (inhibition test) or $100 \mu \mathrm{l} \mathrm{NaCl} 0.8 \%$ (control). In addition to this routine inhibition test, we incubate the patient's plasma with Ch1- or Rg1-negative plasma. After $30 \mathrm{~min}$. incubation at $37{ }^{\circ} \mathrm{C}$ we tested all probes by IAT column technique against 3 test cells.

An initially positive antibody screen test becoming negative after inhibition with normal plasma indicates the presence of Anti-Ch1 or Anti-Rg1. The result of the additional inhibition test with Ch1-negative or Rg1-negative plasma respectively enables to identify exactly Anti-Ch1 and/or Anti-Rg1.

Results: We tested 15 patients with known or suspected Anti-Ch1 or Anti-Rg1, respectively. The specificities of the previously unknown antibodies were confirmed by additional serological and molecular biological analyses of the corresponding antigens.

We found 4 Anti-Rg1 and 11 Anti-Ch1. In all cases the Extended Inhibition Test was able to predict the correct specificity Anti-Ch1 or Anti-Rg1.
Conclusion: Using Ch1- and Rg1-negative Plasma in the Extended Inhibition Test Anti-Ch1 and Anti-Rg1 can be identified and discriminated with a simple serological method. We think, cost- and time-consuming molecular testing can be saved due to the accuracy of the test.

\section{IMH-P18}

\section{Two novel mutations of the Rh-Associated Glycoprotein causing $\mathrm{Rh}_{\text {null }} / \mathrm{Rh}_{\text {mod }}$ phenotypes}

${ }^{*}$ C. Weinstock ${ }^{1}$, F.F. Wagner ${ }^{2}$, J.M. Rox ${ }^{3}$, M. Anliker ${ }^{1}$, H. Schrezenmeier ${ }^{1}$, I. von Zabern ${ }^{1}$

${ }^{1}$ Institute of Clinical Transfusion Medicine and Immunogenetics UIm, German Red Cross Blood Service Baden-Württemberg - Hessen, Institute UIm, University of UIm, UIm, Germany

${ }^{2}$ German Red Cross Blood Service NSTOB, Institute Springe, Springe, Germany

${ }^{3}$ Institute of Transplantation Diagnostics and Cellular Therapeutics, University of Düsseldorf, Düsseldorf, Germany

Background: Expression of RhD and RhCE on red blood cells (RBC) requires the presence of the Rh-Associated Glycoprotein (RhAG). The $3 \mathrm{Rh}$ proteins form a complex in the cell membrane together with other membrane structures including Glycophorin B (GPB). The rare $\mathrm{Rh}_{\text {null }}$ phenotype results from silenced $R H D$ and $R H C E$ genes (amorph type), or from a silenced $R H A G$ gene (regulator $\mathrm{Rh}_{\text {null }}$ ). In addition, several non-silencing mutations of the $R H A G$ gene are known, which are associated with a decreased expression of RhAG leading to a weak expression of $\mathrm{RhD}$ and $\mathrm{RhCE}\left(\mathrm{Rh}_{\bmod }\right)$.

Typing of Rh and MNS antigens was done with a routine tube method. Methods: The presence of RhAG on the RBC was tested with two specific monoclonal antibodies in the indirect antiglobulin test (IAT) with a column agglutination method (Biorad). Eluates (acid method) prepared from RBC after adsorption with polyclonal antisera were tested in the IAT (column agglutination; Capture method, Immucor). $R H D$ and $R H C E$ alleles were determined by in-house PCR. For investigation of the RHAG genes, genomic DNA was amplified and sequenced.

2 Propositi with an apparent $\mathrm{Rh}_{\text {null }}$ phenotype were investigated. Both were negative for RhD and RhCE antigens and for RhAG antigen by standard methods. Propositus 1 carried the alleles $R H D, R H C, R H c$, and $R H e$. Sequencing the $R H A G$ gene revealed heterozygosity for the previously described splice site mutation c. $157+1 \mathrm{G}>\mathrm{A}$ and for a novel nucleotide substitution in exon 7 (c.976 G>A; p.(Asp326Asn), transmembrane/intracellular border).

Results: After adsorption/elution, Anti-D and anti-c, respectively, were detectable. Neither anti-C nor anti-e could be demonstrated. $N$ expression was normal, expression of antigen s was decreased. Propositus 2 carried the alleles $R H D, R H C$, and $R H e$. At the RHAG locus he was heterozygous for the splice site mutation c. $157+1 \mathrm{G}>\mathrm{A}$ and for a novel nucleotide substitution in exon 2 (c.172T $>$ A; p.(His58Tyr), transmembrane section). Both mutations were also present in his parents. No RBC for absorption and elution were available. We describe 2 propositi with an apparent $\mathrm{Rh}_{\text {null }}$ phenotype when standard serological typing methods were applied. Both individuals were heterozygous for the previously described mutation c. $157+1 \mathrm{G}>\mathrm{A}$ and a second, novel nucleotide substitution.

Conclusion: Homozygosity for c. $157+1 \mathrm{G}>\mathrm{A}$ is known to result in complete absence of RhAG. Therefore, the two novel mutations caused an excessive loss of RhAG expression. Propositus 2 could not be tested by adsorption/elution. Propositus 1 expressed RhD (DEL) and Rhc weakly, as demonstrated by adsorption/elution. Substitution of transmembrane amino acids in $\mathrm{RhD}$ is typically for weak D. Due to the similar structure of RhD and RhAG, the low expression of RhAG and, thus, RhD/ $\mathrm{CE}$ antigens, is plausible for these mutations. Low expression of RhAG and $\mathrm{RhD} / \mathrm{CE}$ antigens may be important for allele carriers with respect to immunization in case of transfusion. 


\section{IMH-P19}

Genotyping of the Vel Blood Group by PCR-SSP

${ }^{*}$ A. Reill', B. Flesch ${ }^{2}$, B. Just ${ }^{1}$, J. Bux ${ }^{1}$

${ }^{1}$ DRK-Blutspendedienst West, Hagen, Germany

${ }^{2}$ DRK-Blutspendedienst West, Bad Kreuznach, Germany

Background: The blood group Vel was already described in 1952, but the molecular basis remained unclear. Vel- individuals are extremely rare and since anti-Vel can cause severe haemolytic transfusion reactions, easy Vel antigen typing is an important task in transfusion medicine. Lack of suitable typing sera hampered large scale screening for Vel- blood donors. Quite recently, the genetic background of Vel could be elucidated. Homozygosity for a 17 nucleotide deletion in the third exon of SMIM1 is responsible for the Vel- phenotype. Here, we present an easy method for Vel genotyping using PCR-SSP.

Methods: Four PCR primers were designed to form 3 mixes. A consensus sense primer was combined with a consensus antisense primer, a Vel-positive antisense primer including the deletion and a Vel-negative antisense primer spanning the deletion, respectively. The consensus mix yields a larger product in Vel+ individuals, a smaller one in Vel- individuals or two products in the heterozygous state. Primers as described by Ballif et al. were used for DNA sequencing of the third and fourth exon of the small integral membrane protein 1 (SMIM1).

Results: DNA samples of 12 Vel- patients or blood donors were available for genotyping. All of them exhibited homozygosity for the newly described 17 nucleotide deletion in the SMIM1 gene (Ballif et al. 2013, Storry et al. 2013, Cvejic et al. 2013). As expected, the son of a Vel- patient proved to be heterozygous. All results of serological typing, PCRSSP and DNA sequencing were in perfect accordance.

Conclusion: We describe an easy PCR method that will facilitate large scale screening for Vel-negative blood donors. Screening using only one mix of primers seems to be feasible.

\section{IMH-P20}

\section{Genotyping rare blood groups by PCR-SSP}

\section{${ }^{*} P$. Bugert, G. Rink, E.A. Scharberg, K. Janetzko}

DRK-Blutspendedienst Baden-Württemberg - Hessen, Institut für Transfusionsmedizin und Immunologie, Mannheim, Germany

Background: The identification of antibody specificity in patients with antibodies against high and low prevalence blood group antigens is a challenge in transfusion medicine. In cases with unclear serological results molecular methods can provide the patient's genotype and important information for the antibody identification. Therefore, we developed PCR methods based on the use of sequence-specific primers (PCR-SSP) for typing of rare blood groups.

Methods: PCR-SSP methods were developed for detection of $\mathrm{Di}(\mathrm{a} / \mathrm{b})$, $\mathrm{Yt}(\mathrm{a} / \mathrm{b}), \operatorname{In}(\mathrm{a} / \mathrm{b}), \operatorname{Js}(\mathrm{a} / \mathrm{b}), \operatorname{Lu}(8 / 14), \operatorname{LW}(\mathrm{a} / \mathrm{b}), \operatorname{Sc}(1 / 2), \mathrm{Kn}(\mathrm{a} / \mathrm{b}), \mathrm{McC}(\mathrm{a} / \mathrm{b})$ $\mathrm{Sl}(1 / 2), \mathrm{Yk}(\mathrm{a} / \mathrm{-})$ alleles. Patients and blood donors with serological evidence for or with assumed presence of at least one of the listed blood group antigens were typed by PCR-SSP.

Results: For PCR-SSP typing we selected 177 samples (41 patients, 136 donors). From the genotyping results we could deduce blood group phenotypes as follows: 11 patients with $\mathrm{Kn}(\mathrm{a}-\mathrm{b}+) ; 2$ patients and 1 donor with Sl:1,2; 2 patients with Sl:2,2; 1 patient with $\mathrm{Js}(\mathrm{a}+\mathrm{b}-)$; 3 donors with $\mathrm{Yt}(\mathrm{a}-\mathrm{b}+)$. In addition we diagnosed the $\mathrm{Yk}(\mathrm{a}-)$ phenotype in 7 donors and 9 patients. With regard to low prevalence antigens we identified 5 donors positive for Lu14, 2 donors and 1 patient positive for $\mathrm{LW}(\mathrm{b}), 2$ donors positive for $\mathrm{Sc} 2$ and 1 patient positive for $\mathrm{McC}(\mathrm{b})$.

Conclusion: Genotyping is a very useful tool to determine the antibody specificity in patients with unclear antibodies against high prevalence antigens. It contributes to a better supply of these patients with compatible blood units or to a better clinical risk assessment if serologically incompatible blood units have to be transfused. Identifying antibodies against low prevalence antigens can be important in prenatal diagnostics.
IMH-P21

\section{Distribution of $R H D$ variants in a patient population in Saxony-Anhalt}

\section{${ }^{*}$ S. Jorks ${ }^{1}$, U. Schwab ${ }^{1}$, A. Engelskircher ${ }^{1}$, A. Döscher ${ }^{2}$, H. Kroll ${ }^{1}$} ${ }^{1}$ Red Cross Blood Transfusion Service NSTOB, Institute for Transfusion Medicine Dessau, Dessau, Germany

${ }^{2}$ Red Cross Blood Transfusion Service NSTOB, Institute for Transfusion Medicine Oldenburg, Oldenburg, Germany

Background: The $\mathrm{Rh}$ system is the most immunogenic and clinically important protein-based blood group antigen system. Single nucleotide polymorphisms in the transmembrane domain of the RHD gene lead to weak protein expression. Other mutations or hybrid genes between $R H D$ and RHCE are responsible for the expression of most partial Ds. Since some patients with partial or weak D can form anti-D antibodies these variants should be well characterized. In this study, we systematically analyzed patients with weak agglutinations in serologic RhD typing by RHD genotyping.

Methods: RhD phenotyping of about 65,000 patients of the immunohematologic laboratory in Dessau was performed by tube agglutination technique or automated blood typing system Tango optimo (Biotest) with two different monoclonal anti-D antibodies that did not react with RhD category VI. All samples that showed agglutinations of $2+$ or less or different reaction strengths between the sera and on direct inquiry from hospitals were analyzed by $R H D$ genotyping. DNA was extracted from EDTA-anticoagulated blood. RHD genotyping was performed by PCR with sequence-specific primers (CDE SSP, weak D SSP) and in selected cases by direct Taq cycle sequencing using BigDye terminators.

Results: A total of 359 patients with weakend anti-D reactions were analyzed. $R H D$ variants were detected in 354 (98.6\%) patients. Seventeen different weak D types and six different partial $R H D$ alleles were found. In detail, the following $R H D$ genes were identified: weak D type 1: 198 (55.2\%), weak D type 2: $87(24.2 \%)$, weak D type $3: 41(11.4 \%)$, D cat VII: $3(0.8 \%)$, weak D type $4.0 / 4.1$, weak D type 5 , weak D type 14 , and weak D type 42 each $2(0.6 \%)$, weak D type 18 , weak D type 20 , weak D type 25 , weak D type 31 , weak D type 41 , weak D type 49 , DAU: 1 $(0.3 \%)$, DNB, DHMi, and DFR2 each $1(0.3 \%)$. In addition, five new RHD alleles were identified. Weak D type $61: 1(0.3 \%)$, weak D type 63 : $1(0.3 \%)$, weak D type $64: 3(0.8 \%)$, RHD(A273V): $1(0.3 \%)$, and RHDCE(3,4)-D: $1(0.3 \%)$.

Conclusion: The study demonstrates that weak reactions in RhD phenotyping were almost always $(98.6 \%)$ associated with genetically defined $R H D$ variants. We think that a $2+$ agglutination is a reasonable threshold to initiate $R H D$ genotyping. Seventeen different weak $D$ types, five different partial $R H D$ alleles, and one hybrid allele were found. However, we did not find an association between strength of agglutination with certain weak or partial $R H D$ alleles. Thus, $R H D$ genotyping should be routinely integrated into the blood group typing strategy for patients to avoid unnecessary immunizations.

\section{$\mathrm{IMH}-\mathrm{P} 22$}

\section{Prevalence and specificities of red cell alloantibodies}

\section{${ }^{\star}$ D. Marandiuc, R. Conradi, S. Runkel, D. Kreutzmann,} W.E. Hitzler

Universitätsmedizin Mainz, Transfusionszentrale, Mainz, Germany

Background: The blood group antibodies (abs) can raise major problems for compatible blood transfusions. We retrospectively reviewed our transfusion records from 2010 to identify new alloimmunized individuals. In that year we typed and screened 8,280 different patients. In this study we included only new detected abs by known patients and all abs from patients new to us.

Methods: Blood grouping was performed manually on bioplates and with gel column agglutination. Antibody screening and identification were also manually performed with Bio-Rad ${ }^{\circledR}$ coombs ID cards and antibody identification panels from Bio-Rad ${ }^{\circledR}$, Medion Diagnostics Data-Cyte ${ }^{\circledR}$ 
Plus and Immucor Gamma ${ }^{\circledR}$ Panocell ${ }^{\circledR}$. The Wra positive reagent red cells were from Sanquin Reagents. Positive screens with inconclusive results and auto-ab were excluded. We included coombs reactive anti-M, anti-P1 and anti-Lewis abs.

Results: We found 322 patients with abs, 40\% men and 60\% women $43.78 \%$ of the patients were already known in our database. 309 patients had 383 coombs reactive Abs and 13 patients only one cold reactive $\mathrm{Ab}$ The most frequent $\mathrm{Ab}$ was anti-D with $16.97 \%$, followed by anti-Lua $13.83 \%$, anti-E $12.01 \%$, anti-K $12.01 \%$, anti-C 6.52\%, anti-Lea 5.22\%, anti-Wra $5.22 \%$. 246 patients $(76,39 \%$ ) had only one ab, 50 patients had 2 abs, 11 patients had 3 abs and 2 patients had 4 abs. The most frequent allo-ab combinations were C/D for 23 patients and $\mathrm{c} / \mathrm{E}$ for 10 patients. 49 patients were followed-up after a longer period of time (6 months or more). 37 of them still had their abs. Abs most frequent found were anti-D (8), anti-K (8), anti-E (4), anti-Fya (4), anti-Lua (3).

Conclusion: As in most other studies, the incidence of alloimmunisation against red blood cell antigens was higher for female patients. Here the immunization through pregnancy could play an important part. The majority of the patients had a single ab. The immunogenity of $\mathrm{D}$ antigen was again underlined, corresponding to the high incidence of the found anti-D abs.

\section{IMH-P23}

\section{Novel A variant Allele of the ABO Blood Group Gene (A101 Var V87E)}

\section{${ }^{*} D$. Marandiuc, A. Döscher, R. Conradi, G. Maccagno, W.E. Hitzler}

Universitätsmedizin Mainz, Transfusionszentrale, Mainz, Germany

Background: The blood transfusion services have to assure the safety of the blood supply. Therefore, it is important to correctly determine the blood group of donors, as of patients to make a compatible blood transfusion possible. Routinely, we perform an extended blood group typing for the first two donations. For one donor we obtained discrepant results for the forward compared with the reverse ABO blood grouping. To clarify our findings the sample was sequenced and a novel A variant allele was discovered.

Methods: Routinely we determine donor ABO blood group with an automated serological method on Immucor Gamma ${ }^{\circledR} \mathrm{Neo}^{\mathrm{TM}}$. This instrument uses a microplate agglutination technique. Standard ABO DNA typing was performed using a PCR-SSP test from BAG Healthcare (BAGene ABO-TYPE variant). ABO sequencing was performed with Taq-polymerase cycle sequencing using fluorescent-labeled dye terminator reactions. The sequencing data was analyzed and compared to known reference sequences with MacVector ${ }^{\circledR}$ Software.

Results: In the serological forward $\mathrm{ABO}$ typing we found a weak reaction to anti-A and anti-AB reagents and in the the reverse typing, a weak reaction to B erythrocytes. This suggested a variant A blood group. To identify this variant we performed a DNA typing which indicated an ABO*O01/ A101 genotype. Sequencing exon 6 showed for the first haplotype a deletion of nucleotide $\mathrm{G} 261$ as expected for $\mathrm{ABO} * \mathrm{O} 01$. The second haplotype showed a T257A nucleotide substitution. Consequently, a valine amino acid is replaced by a glutamic acid [V87E] in the ABO transferase. This allele was named A101 Var V87E.

Conclusion: The inconsistency between serological results (suggesting a deficient $\mathrm{A}$ antigen) and molecular results (genotype $\mathrm{ABO} * \mathrm{O} 01 / \mathrm{A} 101$ ) was clarified through sequencing of exon 6 . In addition to the expected ABO*O01 haplotype, a new nucleotide substitution [T257A] was discovered in the second haplotype, leading to an amino acid exchange [V87E] for the $\mathrm{N}$-acetylgalactosamine-transferase. The genotype of the donor was described as ABO*O01/A101 Var V87E.

\section{IMH-P24 \\ RHCE Hybrid Gene as Cause for -D- Haplotype: Confusion in Mother-Child Blood Group typing}

${ }^{\star} R$. Conradi ${ }^{1}$, A. Doescher ${ }^{2}$, D. Marandiuc ${ }^{1}$, G. Maccagno ${ }^{1}$, A. Jung ${ }^{1}$, W.E. Hitzler ${ }^{1}$

${ }^{1}$ Universitätsmedizin Mainz, Transfusionszentrale, Mainz, Germany ${ }^{2}$ DRK Blutspendedienst NSTOB, Oldenburg, Germany

Background: As part of routine postnatal care, we tested the blood type of a mother and her newborn child. We typed the mother as B CCD.ee $(\mathrm{D}+\mathrm{C}+\mathrm{c}-\mathrm{E}-\mathrm{e}+)$ and the newborn as $\mathrm{O}$ ccD.EE $(\mathrm{D}+\mathrm{C}-\mathrm{c}+\mathrm{E}+\mathrm{e}-)$. In this presentation we describe the extended range of our investigations to elucidate this constellation, which at a first glimpse seems impossible.

Methods: Serological blood group typing was done by standard column agglutination testing (BioRad, ID-System). DNA typing of HLA A-, B-, C-, DRB1- and DQB1 low resolution was done with PCR-SSO (Invitrogen RELI) and PCR-SSP (BAG, BAGene) kits. DNA typing of RHD and RHCE was performed with PCR-SSP (BAG, BAGene) kits. Sequencing of RHD and RHCE gene exons 1 to 10, including intron/exon borders, was done by direct taq cycle-sequencing using BigDye terminators v1.1. Quantification of RHD and RHCE sequences by real-time PCR, spanning exons 3 to 7, was used for determination of RHCE mutations masked by normal RHCE alleles in trans.

Results: We initially thought at a sampling mistake and then later at an egg donation. Reruns of the blood typing with new blood samples confirmed the first results. A RHD and RHCE DNA typing sustained the serological results. HLA typing of mother and child confirmed their relation and excluded the egg donation or the eventually switch at birth. Sequencing of mothers' and childs' DNA showed wildtype sequences for the RHD gene. Examination of the RHCE gene revealed a RHCe genotype for the mother and RHCcE for the child. The calculated ratio of RHD to RHCE sequences in exons 3 to 7 was $2: 1$ for the mother and $3: 1$ for the child.

Conclusion: The RHD: RHCE ratio indicates a RHCe-D(3-7)-Ce hybrid gene for the maternal DNA. Most probably the resulting haplotypes are Cde and -D-. By inheritance of the hybrid gene to the child, the expressed RhcE antigens are solely of paternal orign. This kind of constellation makes the different antigen expression of mother and child possible. Further experiments are necessary to confirm these results, e.g. haplotype-specific sequencing of cDNA.

Fig. 1

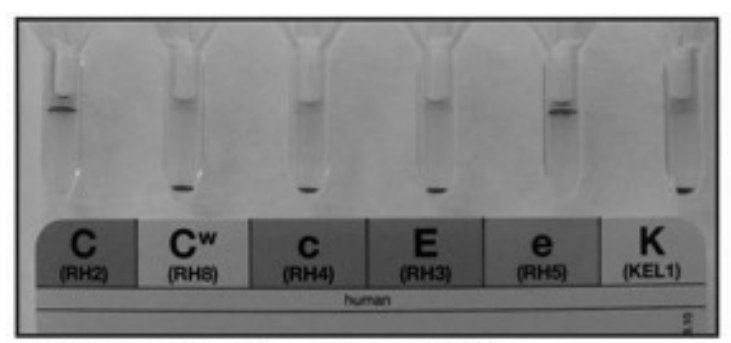

\section{Rh-formula of mother: CCD.ee}

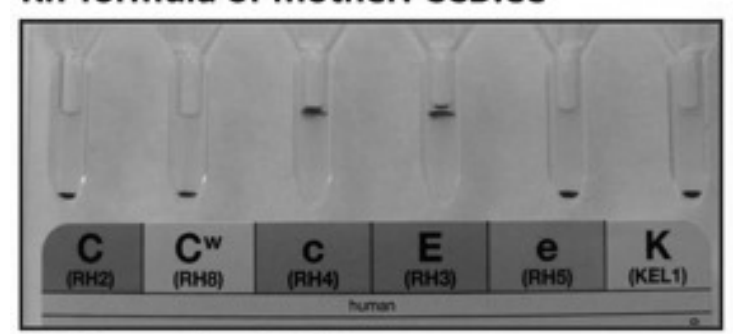

Rh-formula of child: ccD.EE 


\section{IMH-P25}

\section{Adoptive immunotherapy as therapeutic option to treat human papilloma virus associated tumours}

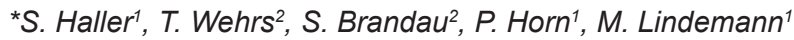 \\ ${ }^{1}$ Universitätsklinikum Essen, Institut für Transfusionsmedizin, \\ Transplantationsdiagnostik und F\&E, Essen, Germany \\ 2Universitätsklinikum Essen, Klinik für Hals-Nasen-Ohrenheilkunde / \\ Forschung, Essen, Germany
}

Background: Human Papilloma Viruses (HPVs) are known to cause malignant transformation. The high-risk type HPV16 is associated with cervical cancer and head and neck squamous cell cancer. HPV16-positive tumour cells are supposed to carry the oncogenes E6 and E7 as antigens. These markers may provide a target for an adoptive immunotherapy. For therapeutic success, it is necessary to expand HPV-specific peripheral blood mononuclear cells (PBMCs) to an adequate amount. Expanded cells still have to be functional, therefore, methods for quantification and characterisation have to be optimised.

Methods: HPV-specific PBMCs can be analysed via enzyme-linked immunospot (ELISpot) assay on the one hand and europium-2,2 $: 6^{`}, 2^{\prime \prime}$-terpyridine-6,6"-diacarboxylate (EuTDA) cytotoxicity assay on the other hand. Cytokine producing PBMCs can be identified and quantified on a single cell level with the ELISpot assay. To quantify HPV16-specific cells, the interferon- $\gamma$ (IFN- $\gamma$ ) ELISpot assay has been optimised in our laboratory. Currently, ELISpot assays to measure the HPV16-specific production of interleukin 10 (IL-10), granzyme B and perforin in healthy persons are optimised. Furthermore, the cytotoxic activity of HPV16-specific T lymphocytes against HPV16-positive tumour cells will be tested with an EuTDA cytotoxicity assay. To set up the test, a HPV16-positive tumour cell-line, UPCI-SCC-090, is compared with a HPV16-negative one, UT-SCC-050.

Results: The frequency of HPV16-specific cells producing IFN- $\gamma$ in 33 healthy persons is quite low (1/50,000 for HPV16 E6 and 1/200,000 for HPV16 E7) with a median IFN- $\gamma$ spot number of 2.0 for HPV 16 E6 and 0.5 for HPV 16 E7, respectively, out of 100,000 cells. ELISpots to detect IL-10, granzyme B and perforin production will be optimised soon. The EuTDA cytotoxicity assay was established using the cell line K562. With this cell line, the specific lysis of labeled target cells K562 by freshly isolated PBMCs was determined. Currently, the assay procedure is optimised for HPV16-positive and -negative tumour cell lines, UPCI-SCC-090 and UT-SCC-050, respectively.

Conclusion: As soon as all methods to analyse HPV16-specific PBMCs are optimised, further data can be acquired for healthy persons as well as patients affected with HPV16-positive tumours. These data will be compared with each other and also with healthy persons vaccinated against HPV. The comparison of healthy non-vaccinated persons with persons vaccinated against HPV might reveal a new cohort of probands, whose PBMCs can be used to set up the expansion protocols. As soon as adequate expansion protocols are ready, the cytotoxic activity of expanded HPV16-specific T lymphocytes will be tested.

\section{IMH-P26}

Wilms' tumor 1 (WT1)-specific T lymphocytes as a tool for adoptive immunotherapy in acute myeloid leukemia

\section{${ }^{*}$ T. Danielzik' , E. Hermann ${ }^{2}$, U. Buttkereit ${ }^{2}$, D. Beelen², P. Horn ${ }^{1}$,} M. Lindemann ${ }^{1}$

${ }^{1}$ Universitätsklinikum Essen, Institut für Transfusionsmedizin, Essen, Germany

${ }^{2}$ Universitätsklinikum Essen, Klinik für Knochenmarktransplantation, Essen, Germany

Background: The Wilms' tumor 1 (WT1) antigen is involved in cell differentiation and haematopoiesis. It is known to be highly expressed in hematopoietic malignancies such as acute myeloid leukemia (AML) and thus many current studies suggest the use of WT1 peptide vaccination. Successful peptide vaccination is questionable as patients suffering from
AML have a compromised immune system. We started a study that focuses on the production of WT1-specific T lymphocytes that may provide a more useful tool, as specific and fully functional cells derived from patients themselves should be transfused.

Methods: We isolated peripheral blood mononuclear cells of healthy persons and AML patients pre and post transplantation to analyse differences in the frequency of WT1-specific T lymphocytes. Isolated cells were tested upon WT1 peptide recognition and analysed by enzyme-linked immunospot (ELISpot) assay. By ELISpot assay, cytokine producing cells can be identified and quantified on a single cell level. The WT1-specific production of different cytokines (Interferon- $\gamma$, Interleukin-10) was measured, single cells were counted and frequencies determined. Furthermore, $\mathrm{T}$ cell functionality in patients pre and post transplantation is also tested in ELISpot with the cytolytic enzymes granzyme B and perforin. Cytotoxic activity of WT1-specific T lymphocytes against different AML specific cell lines is tested in a europium release (EuTDA) assay.

Results: Quantification of WT1-specific T lymphocytes producing IFN- $\gamma$ resulted in a frequency of $0.0010 \%$ in healthy controls and frequencies of $0.0014 \%$ and $0.0115 \%$ in patients pre- and post-transplantation, respectively. In comparison, IL-10 production was much higher in patients post-transplantation $(0.3575 \%)$ but seems to be absent in patients pre-transplantation.Cytolytic enzymes were not produced in healthy controls but in patients pre- and post-transplantation in a lower amount $(0.0060 \%$ for granzyme B). Perforin production post-transplantation was slightly increased with a frequency of $0.0105 \%$. In EuTDA assay, target cells (TC) were incubated with TDA ligand that enters the cell. Upon lysis of TC by WT1 specific effector cells, ligand is released and later coupled with fluorescent europium. The EuTDA assay is currently optimised for specific lysis in different AML cell lines

Conclusion: Specific T cells of AML patients post-transplantation tend to produce more cytokines upon WT1 specific stimulation than AML patients pre-transplantation and healthy controls. Post-transplantation patient cells seem to be more active as the proinflammatory response is generally increased. These findings suggest the use of patient WT1-specific $\mathrm{T}$ lymphocytes postHCT as a tool for cellular immunotherapy. Therefore cells are further tested for cytotoxic activity to ensure their lytic potential to prevent relapse.

\section{IMH-P27 \\ Erythrocyte alloantibody in transfusion dependent thalassemia patients: Proportion and related factors}

\section{*A. Kurniawan1, D. Atmakusuma², L. Sukrisman²}

${ }^{1}$ Faculty of Medicine, University of Pelita Harapan, Internal Medicine, Tangerang, Indonesia

${ }^{2}$ Faculty of Medicine, University of Indonesia, Hematology and Medical Oncology, Jakarta, Indonesia

Background: In transfusions dependent thalassemia patients who have got repeated transfusion for a period of time, the hemoglobin level after transfusion could not be maintained appropriately to be expected. The production of erythrocyte alloantibody in transfusion dependent thalassemia patients has been reported before. These antibodies were probable related to the failure on maintaining hemoglobin level after transfusion. the aims of this study was To find proportion and related factors of erythrocyte alloantibody production in transfusion dependent thalassemia patients.

Methods: Cross sectional study of adult transfusion dependent thalassemia patient without others autoimmune disease at Hematology and Medical Oncology outpatient clinic in Cipto Mangunkusumo hospital from July to December 2012 was done. The specimen was subjected to erythrocyte alloantibody evaluation by column gel agglutination technique. Eleven cell reagent panels were used in screening and identification of alloantibody. Positive alloantibody is defined as positivity of indirect anti-globulin test. Statistic analysis was done between erythrocyte alloantibody production and several factors related such as sex, age of initial transfusion, type of rhesus, history of splenectomy, and types of packed red cell. 
Results: From 88 subjects, there were $37,5 \%$ thalassemia patients that did not maintain hemoglobin level after transfusion. From 33 of those subjects, there were $78,6 \%$ subjects with alloantibody. Positive alloantibody did not correlate to sex, age of initial transfusion, type of rhesus, history of splenectomy, and types of packed red cell.

Conclusion: The proportion alloantibody production in adult thalassemia patients that failure on maintaining hemoglobin level were 78,6\%. Positive alloantibody did not correlate to sex, age of initial transfusion, type of rhesus, history of splenectomy, and types of packed red cell.

\section{IMH-P28 \\ $\mathrm{Rh}-\mathrm{D}$ determination with molecular genetic methods: Useful and necessary?}

\section{*K. Henneberg-Quester, I. Schumacher}

Universitätsklinik Essen, Institut für Transfusionsmedizin, Essen, Germany

Background: Since the 1990ies several working groups in transfusion medicine determine the genetic circumstances of the Rh-genes with the new possibilities of PCR-tests. Several amazing findings especially concerning the gene Rh D were found. In different groups of humans the genetic information is transferred in different manners in the antigens of the erythrocyte surface. Mostly in Caucasians the type dd is based upon a deletion of the gene $\mathrm{D}$, in people of African origin the gene Dpsi as a result of a nonsense mutation is followed by serological $\mathrm{D}$ negative and in people from Asia it is followed by a hybrid allele.

Methods: Since 1997 diagnostic test kits for PCR-SSP were distributed Initially we used the kit of the University of Innsbruck, in the following years the commercial kits provided by InnoTrain and BAG (DNA preparation, cycling conditions and detection as described by the manufacturer). Results: Amongst 100 donors with the subgroups Ccddee and ccddEe 8 donors were D-positive (Table 1). Positive donors with the serological type dd are rare, but consistently detectable.

To avoid anti-D development donors with modified occurrence of the D-antigen should be reported as D-positive (Gassner C. et al.). In the open society of central Europe mixtures happen due to migration and genetic drift. These circumstances occur in any transfusion center, even small ones. Since beginning of the tests we avoided 87 transfusions of D-positive erythrocytes to D-negative patients.

Conclusion: Patients and especially girls and women in child-bearing age in case of transfusion should get only blood tested by molecular genetic testing methods in order to avoid alloimmunisation. Whether even the most rare appearance of $\mathrm{D}$ positive donors in ccddee population needs to be determined, has to be discussed. Reference: Gassner C. et al. Presence of RHD in serologically D-, C/E+ individuals: a European multicenter study. Transfusion 45, 2005

\begin{tabular}{|l|l|l|l|}
\hline $\begin{array}{l}\text { Serological } \\
\text { result }\end{array}$ & PCR result & $\begin{array}{l}\text { Donations } \\
\text { after PCR }\end{array}$ & $\begin{array}{l}\text { Donations } \\
\text { before PCR }\end{array}$ \\
\hline & & & \\
\hline Ccddee & D pos & 25 & 18 \\
\hline Ccddee & D pos & 7 & 26 \\
\hline Ccddee & D pos & 32 & 19 \\
\hline Ccddee & D pos & 4 & 0 \\
\hline Ccddee & D pos & 0 & 0 \\
\hline Ccddee & D-CE(2-9)-D & 5 & 0 \\
\hline Ccddee & D(K409K DEL & 3 & 0 \\
\hline ccddEe & Dweak type 2 & 11 & 2 \\
\hline
\end{tabular}

Table 1: Determination of D with molecular genetic methods (PCR)

\section{IMH-P29 \\ Drug-induced immune hemolysis: one center's experience}

\section{${ }^{*} B$. Mayer, R. Genth, T. Bartolmäs, S. Yürek, A. Salama}

Charité - Universitätsmedizin Berlin, Institut für Transfusionsmedizin, Berlin, Germany

Background: Drug induced immune hemolysis is difficult to diagnose and the true incidence is obscure. During the last 15 years we identified a total of 60 patients with drug-induced immune hemolytic anemia (DIHA). Methods: Serological tests were performed with standard techniques using gel cards. Drug-dependent antibodies were investigated in the presence and absence of the drug and / or their ex vivo antigens (urine of patients treated with the drug).

Results: Hemolysis was acute and severe in all patients. Outcome was fatal in ten patients $(16,6 \%)$. The direct antiglobulin test (DAT) was positive in 53 patients (88\%) with $\mathrm{C} 3 \mathrm{~d}( \pm \operatorname{IgG} \pm \operatorname{IgM} \pm \operatorname{IgA})$, in five patients with $\mathrm{IgG}$, in one patient with $\operatorname{IgA}$ and negative in one patient who has been investigated six month following the hemolysis.

Drug dependent antibodies to 13 different drugs with the following specificities were identified: Diclofenac (19), Piperacillin (11), Oxaliplatin/ Carboplatin (10), Ceftriaxon (9), Cefotaxim (2), Rifampicin (2), radiographic contrast media (2), Cotrimoxazol (1), Clindamycin (1), Ibuprofen (1), Etoricoxib (1), 5-Fluorouracil (1).

Conclusion: DIHA is a rare but potentially life threatening autoimmune hemolytic anemia. The key serological finding is a positive DAT, mainly with Anti-C3d. Based on our results, the most common drugs causing DIHA were antibiotics. The most common single drug identified was Diclofenac, followed by Piperacillin, Oxaliplatin and Ceftriaxon. Together these four drugs counted for $80 \%$ of all cases. The spectrum of the drugs associated with DIHA has changed considerably during the last decades.

\section{IMH-P30 \\ Clinical relevance of $\mathrm{RBC}$ autoantibodies occurring in pregnancy}

\section{${ }^{*}$ B. Mayer, E. Puschkina, A. Salama}

Charité - Universitätsmedizin Berlin, Institut für Transfusionsmedizin, Berlin, Germany

Background: Autoantibodies to RBCs are considered to occur only rarely in pregnancies. In addition, the significance of these autoantibodies are conflicting, ranging from insignificant to serious hazard to mother and child. We report our data on pregnant women with autoantibodies who were investigated at our university hospital during the last 12 years.

Methods: Serological tests were performed with standard techniques using gel cards. All pregnant women with positive antibody screen were analyzed. None of the women had significant autoimmune hemolytic anemia prior to pregnancy.

Results: A total of 381 pregnant women with antibodies were analysed. Of these, $318(83,5 \%)$ had alloantibodies, $49(12,9 \%)$ had autoantibodies and $14(3,7 \%)$ had auto- and alloantibodies. Of the 63 patients with autoantibodies, 60 patients had warm reactive autoantibodies, two patients had cold reactive autoantibodies and one patient had both, warm and cold reactive autoantibodies.

None of the pregnant women with autoantibodies and none of their children developed hemolysis. In one case the DAT was positive in the neonate due to maternal autoantibodies crossing the placenta, but there were no signs of hemolysis in the infant and consecutively no treatment was necessary.

Conclusion: Autoimmunization to RBCs may infrequently occur during pregnancies. These autoantibodies can largely considered to be benign and do not cause significant hemolysis. However, regular monitoring during pregnancy is indicated to exclude concomitant alloimmunization. 


\section{IMH-P31}

No increase in fetal platelet counts due to repeated intrauterine IgG infusions in fetal alloimmune thrombocytopenia

\section{G. Giers ${ }^{1}$, H. Lorenz ${ }^{2}$, B. Tutschek ${ }^{3}$, ${ }^{\star} F$. Wenzel ${ }^{4}$}

${ }^{1}$ Universitätsklinik Düsseldorf, IHTM, Düsseldorf, Germany

${ }^{2}$ Biometry and Statistics, Neuberg, Germany

${ }^{3}$ University Hospital Bern, Department of Obstetrics, Bern, Germany

${ }^{4}$ Universitätsklinik Düsseldorf, ITZ, Düsseldorf, Germany

Background: It is well known that non-specific IgG infusions can suppress antibody-mediated cell destruction. Therefore several studies used this mechanism by maternal intravenous IgG (IVIG) administration for the treatment of neonatal alloimmune thrombocytopenia (FNAIT) showing inconsistent results. We speculated that failure of maternal IVIG could be due to an inactivating modification of the IgG during the active placental transport, and retrospectively studied a cohort of fetuses treated directly with IVIG.

Methods: We retrospectively analyzed data from a cohort of ten fetuses with FNAIT treated by direct fetal immunoglobulin infusion. Fetal treatment was begun between 17 and 25 weeks and continued until 36 weeks of gestation with weekly cordocenteses and fetal immunoglobulin infusions. The fetuses received IVIG (Endobulin ${ }^{\mathrm{a}}$ Immuno $\mathrm{GmbH}$, Heidelberg, Germany) at $1 \mathrm{~g}$ per kg estimated fetal weight.

Results: IgG levels in fetuses increased significantly by IVIG administration from $252 \pm 72 \mathrm{mg} / \mathrm{dl}$ at $21^{\text {st }}$ week of gestation to $1201 \pm 158 \mathrm{mg} / \mathrm{dl}$ at $34^{\text {th }}$ week of gestation. There was no significant change in maternal HPA antibody and $\mathrm{IgG}$ levels. In addition, no significant alterations of the fetal platelet count could be observed: At the time of first sampling the mean fetal platelet count was 18,800 (range 4,000-38,000) vs. 19,000 (range 10,000-38,000) immediately before delivery.

Conclusion: Our retrospective study presents a unique analysis of a historical cohort, contributing to the ongoing debate about the treatment of choice for fetal alloimmune thrombocytopenia and suggesting that IVIG administration does not improve fetal platelet count.

\section{IMH-P32}

\section{Evaluation of the Classic Plus ID GelStation}

\section{*K. Gubbe, A. Karl, M. Schauffel, Y. Scharnagl}

DRK-Blutspendedienst Nord-Ost gemeinnützige $\mathrm{GmbH}$, Plauen, Germany

Background: Time effective and flexible testing with fully automated analysis and documentation are requirements on the automation process in a blood group serology laboratory. The feasibility and economic benefit of the Classic Plus ID GelStation (DIAMED) in a medium sized blood group serology laboratory were evaluated.

Methods: In total 280 ABO group and 98 rhesus type determinations, 327 cross matches and 417 antibody screenings were done manually and in parallel with Classic Plus ID GelStation. The test duration and personnel commitment of the most important test profiles were determined and compared:

Profile 1: ABO blood group, reverse typing and antibody screening

Profile 2: Profile 1 plus rhesus type

Profile 3: Profile 1 plus cross match

Profile 4: ABO blood group, antibody screening and cross match

The profiles were tested with calculated optimal number of samples (see table 1). For cross match testing two erythrocyte concentrates per sample were assumed. In a second step, total test time for the calculated optimal sample size plus one sample was determined.

Results: Between the fully automated and manually processed samples no erroneous results were obtained. Table 1 shows the test duration and personnel commitment of the chosen profiles. Testing with the Classic ID Plus GelStation yielded a time advantage for the operator between 10 and $20 \mathrm{~min}$ per batch and profile. If the profiles 3 and 4 were loaded with calculated optimal capacity plus one sample the test duration increased by $40 \mathrm{~min}$.
Table 1. Test duration and human resource retention

\begin{tabular}{|c|c|c|c|c|}
\hline \multirow{2}{*}{$\begin{array}{l}\text { Profile (opti- } \\
\text { mal sample } \\
\text { size per } \\
\text { batch) }\end{array}$} & \multicolumn{2}{|c|}{$\begin{array}{l}\text { processing with Classic ID } \\
\text { Plus-GelStation }\end{array}$} & \multicolumn{2}{|c|}{ manually processing } \\
\hline & $\begin{array}{l}\text { total test time } \\
\text { (min) }\end{array}$ & $\begin{array}{l}\text { personnel } \\
\text { commit- } \\
\text { ment (min) }\end{array}$ & $\begin{array}{l}\text { total test } \\
\text { time } \\
(\mathrm{min}) \\
\end{array}$ & $\begin{array}{l}\text { personnel com- } \\
\text { mitment (min) }\end{array}$ \\
\hline Profile $1(9)$ & 70 & 25 & 60 & 45 \\
\hline Profile $2(6)$ & 85 & 20 & 60 & 35 \\
\hline Profile $3(8)$ & 140 & 45 & 100 & 65 \\
\hline Profile 4 (8) & 80 & 25 & 65 & 45 \\
\hline
\end{tabular}

Conclusions: Fully automation and the theoretical loading capacity of up to 48 samples, 24 ID-cards and 16 reagents promise higher daily turnover rate and a reduction in staff engagement compared to manual processing of samples.

In contrast we found less time savings than expected. The turnover rate of the Classic ID Plus-GelStation is extremely reduced when urgent samples are loaded or mixed profiles are processed. The low capacity of centrifuge (12 places) and $37^{\circ} \mathrm{C}$ incubator ( 8 places) is the major bottleneck in this regard. Nevertheless it is expected that the use of the Classic Plus automate will reduce operator errors and human labor.

\section{IMH-P33}

Monoclonal antibody therapy with anti-CD44-mAb makes mimicry like the dangerous antibody anti-AnWj in a patient with AML

\section{*I. Trestyanszki, N. Dierks, B. Zimmermann}

DRK-Blutspendedienst NSTOB, Institut Bremen-Oldenburg, Bremen, Germany

Background: The use of monoclonal antibodies in tumor therapy represents a therapeutic benefit for the patients, but at the same time the antibodies can cause a problem for the laboratory tests. We report a case in which the lack of information about such treatment led to dangerous delays and additional costs.

Case Report: A 79-year-old female AML-patient was admitted to hospital with acute gastrointestinal bleeding. The treating hospital found positive antibody screening tests and positive cross matches. On her last hospital stay, she received 2 units of RBCs with a negative antibody test without problems. Blood samples were sent to our laboratory for further examination.

In our laboratory the patient serum reacted positive with all test cells in indirect antiglobulin techniques, except the patient's own cells. Investigations with test cells lacking antigens of high frequency were suggestive of the presence of an antibody with specificity anti-AnWj. AnWj is a high-prevalence antigen (901009) located on an isoform of CD44 glycoprotein. CD44 is present on circulating red cells, B and T lymphocytes, granulocytes and monocytes. Serious hemolytic transfusion reactions have been described.

Results: The complete medical history of the patient was not known. In our further investigation, we found out that she was treated with antiCD44-mAbs in the context of a study. We selected RBCs compatible for the patient's antigens Rh, K, Fy, Jk, M, N, and S. All cross matches were positive. Nevertheless transfusions of several units of RBCs proceeded without any complications after in-vivo hemolysis test and steroid application. 6 weeks later, the antibody screening test was negative.

Conclusion: (A) By the clinical use of anti-CD44-mAb, the immunohematological diagnostics may be affected in patients. The complete medical history avoids extensive examinations and / or misinterpretation, and is important for urgent patient care.

(B) Variable clinical outcomes have been reported in patients with anti-AnWj transfused with AnWj positive RBCs. This is the case of a transfusion without complications by a patient with monoclonal anti-CD44 therapy with AnWj specificity. 
IMH-P34

\section{Fatal neonatal hemolytic disease due to anti-E}

*B. Mayer, A. Pruß, A. Salama

Charité - Universitätsmedizin Berlin, Institut für Transfusionsmedizin, Berlin, Germany

Background: Severe hemolytic disease of the fetus and newborn (HDFN) is usually caused by Anti-D, Anti-K or Anti-c. HDFN associated with anti-E is rare und usually considered to be mild. We report on a newborn with fetal hydrops and fatal hemolysis due to a potent anti-E.

Methods: Serological tests including antibody titers and IgG-subclass determination were performed using standard techniques. Genotyping for RHCE, KEL and GYPB was performed after DNA-extraction using PCR-SSP.

Case report: A 32-year old women presented in her fourth pregnancy for monitoring of a known alloimmunization due to Anti-E, $-\mathrm{K}$ and $-\mathrm{S}$. The first pregnancy of the woman was normal. The second child had a positive DAT due to anti-E but no evidence of HDFN. The third pregnancy ended in fetal death at 8 weeks of gestation.

At the fourth pregnancy, an emergency caesarean section was performed at the 28th week because of fetal hydrops. The newborn had a hemoglobin of $7.5 \mathrm{mg} / \mathrm{dl}$ and a total bilirubin of $9.81 \mathrm{mg} / \mathrm{dl}$. A double volume exchange transfusion was performed on day one after birth, but the baby died on day five.

Results: Antibody-titers at seven weeks' gestation were 512 for anti-E, 256 for anti-K and 128 for anti-S. Titers for anti-K and -S remained unchanged throughout the pregnancy, whereas anti-E titer increased to 1024 at 18 and 28 weeks' gestation, respectively.

After delivery, the direct antiglobulin test (DAT) was strongly positive and maternal Anti-E antibody could be eluted from the neonate's RBC. The child's blood group was determined as $0 \mathrm{Rh}$ pos (D pos), CcEe, K-, S- and confirmed by genotyping. Determination of IgG Anti-E subclasses revealed IgG3.

Conclusion: Anti-E may cause severe HDFN. This is supported by our case and few other cases reported in the literature.

\section{IMH-P35 \\ Crossreactivity of cephalosporin-dependent antibodies involved in immune hemolytic anemia}

${ }^{*}$ T. Bartolmäs, C. Knoth, B. Mayer, A. Salama

Charite, Institut für Transfusionsmedizin, ZTB, Immunhämatologisches Antikörperlabor, Berlin, Germany

Background: Cephalosporins are actually considered to be the most frequently involved compounds in drug-induced immunhemolytic anemia (DIHA). Until now, a little is known about crossreactivities and their significance.

Methods: Serum samples of ten patients who developed DIHA due to ceftriaxone or cefotaxime antibodies were tested in the presence and absence of several widely used cephalosporins and other $\beta$-lactam antibiotics and ex-vivo antigens (urine of patients treated with the antibiotic). Serological testing was performed using standard tests.

Results: Direct antiglobulin tests (DAT) were strongly positive with anti-C $3 \mathrm{~d}$ in all ten cases and with anti-IgG in five cases, too. Eight patients had antibodies to ceftriaxone and 2 patients to cefotaxime. Serum samples of seven of the former patients were also crossreactive in the presence of cefotaxime or its metabolites. Vice versa, the cefotaxime-dependent antibodies crossreacted also in the presence of ceftriaxone or its metabolites. Two antibodies to ceftriaxone and one antibody to cefotaxime showed weak agglutination of RBCs in presence of the majority of the other tested $\beta$-lactam antibiotics. The remaining antibodies showed only weak and isolated reactions with other cephalosporins.

Conclusion: The vast majority of ceftriaxone and cefotaxime drug-dependent antibodies were mutually strongly crossreactive. However, with other cephalosporins and $\beta$-lactam antibiotics these antibodies react not or only weakly. These reactions do not seem to be clinically relevant, although none of the patient has been rechallenged to the causative drug or other cephalosporins.

\section{IMH-P36 \\ Panagglutinating Autoantibodies in Patients with Autoimmune Hemolytic Anemia of Warm Type can be classified by inhibition with monospecific antibodies}

\section{${ }^{*}$ T. Bartolmäs, R. Genth, B. Mayer, A. Salama}

Charite, Institut für Transfusionsmedizin, ZTB, Immunhämatologisches Antikörperlabor, Berlin, Germany

Background: In patients with autoimmune hemolytic anemia (AIHA) of warm type, the causative autoantibodies (aabs) usually belong to the IgG class and less frequently to IgM and / or IgA classes. Rarely, the causative aabs are capable of agglutinating RBCs (panagglutinating autoantibodies) independent of antihuman globulin serum (AHG). These antibodies can not invariably be classified and might be confused with cold agglutinins or falsely attributed to warm IgM aabs. We describe a simple and fast serological technique for the characterization of such antibodies.

Methods: Five patients with severe hemolytic anemia were studied. Serological testing was performed using standard techniques. Eluates from patients' RBC were incubated with monospecific anti-human IgG, IgM and IgA, respectively, and re-tested against normal pooled 0 RBCs using gel cards with monospecific AHG serum.

Results: Routine testing revealed strong autoagglutination of patients' RBCs in all cases and panagglutinating serum samples in three patients. In the latter three patients cold agglutinins with high thermal amplitude were initially suggested. In the remaining two patients IgM warm aabs were suspected. However, inhibition of the eluted aabs by incubation with monospecific antihuman globulins revealed warm aabs of the IgA class in two patients, of the IgM class in two other cases, and of the IgG class in one patient.

Conclusion: Panagglutinating aabs are confusing and can be classified by inhibition experiments using patients' eluates and monospecific antibodies.

\section{IMH-P37}

\section{Comparison of blood group typing using the NEO blood group analyzer and the HEA BeadChip technology}

\section{*O. Meyer, D. Budach, B. Mayer, A. Salama}

Charité - Universitätsmedizin Berlin, Institut für Transfusionsmedizin, Berlin, Germany

Background: Usually, the presence of a blood group antigen is ascertained by an agglutination reaction using monoclonal or polyclonal antibodies that react with the questionable antigen. However, such phenotyping may be complicated by several reasons. In contrast to standard PCR-methods DNA microchip methods provide a fast test procedure and an automated analysis. Here, we report our results of blood group typing by using the HEA BeadChip Kit compared to an automated phenotyping using the NEO blood group analyzer (both Immucor Medizinische Diagnostik GmbH, Germany).

Methods: 512 EDTA-blood samples of healthy blood donors were investigated. Blood group phenotyping was performed using the NEO blood group analyzer and monoclonal and/or polyclonal antibodies against $\mathrm{C}$, c, E, e, K, k, M, N, S, s, Kp(a), Fy(a), Fy(b), Jk(a), Jk(b), Lu(a), Lu(b), $\mathrm{Co}(\mathrm{a}), \mathrm{Co}(\mathrm{b}), \mathrm{Js}(\mathrm{b})$, and Di(a). DNA purification was performed using the automated QIAcube system ( $\mathrm{n}=251$; Qiagen, Hilden, Germany) and the InviGenious system ( $n=261$; Stratec Molecular, Berlin, Germany). Blood group genotyping using the HEA BeadChip Kit was performed as described by the manufacturer.

Results: Automated DNA-isolation was simple and easy using both devices. However, the preparation of the QIAcube system is somewhat complex and may lead to a mix up of samples. In contrast, the preparation of the InviGenious system was clearly arranged, but a considerable amount of samples needed retesting due to low DNA content. The performance of both systems was comparable. The results of blood group pheno- and genotyping were concordant in the majority of patients. Discrepancies were found in two samples for $\mathrm{C}$, and one sample for $\mathrm{k}$. One sample showed 
an indefinite result for $\mathrm{Jk}(\mathrm{a})$ and one further sample was un-evaluable by the HEA BeadChip Kit, probably due to poor DNA quality. The phenotyping results for $\mathrm{Di}(\mathrm{a})$ could not be evaluated, since the antibody was not optimized for NEO system. Finally, one discrepancy was found for s-phenotyping using monoclonal and polyclonal antibodies.

Conclusion: Blood group genotyping is feasible using the HEA BeadChip technology. However, the method is demanding and only applicable for specialized laboratories. Furthermore, it is impossible to type single antigens. In comparison, the NEO system reveals reliable phenotyping results within one hour.

\section{IMH-P38 \\ Frequency of pre-known and newly identified irregular anti-erythrocyte allo- and/or autoantibodies in a regional immunohematological reference laboratory}

\section{${ }^{*}$ C. Baumann, A. Stenzel, G. Bringmann, T. Zeiler}

DRK BSD West Breitscheid, Immunhämatologie, Ratingen, Germany

Background: Patients are frequently treated in in many various therapeutic facilities through the years. However, information about previously identified antibodies often is not passed on, thus increasing the risk of transfusion. Here we present a retrospective analysis on the frequency of newly identified and of previously known, but not reported antibodies in diagnostic samples sent to a regional reference laboratory.

Methods: The laboratory annually receives approximately 12,000 blood samples from many therapeutic facilities for investigation. A huge patient database was built up over the last decades thus giving the chance to fall back on known results of patients if samples are sent in without adequate anamnestic data. 1,000 consecutive samples, collected during January 2012, were included in the analysis. The data collection is based on a wide range of immunohematological methodology including the identification of HTLA antibodies. The following parameters were incorporated: age and gender of patients, antibody specifity, single vs. multiple antibodies, allo- and/or autoantibodies, new findings vs. pre-known findings and results of direct agglutination testing.

Results: $41,8 \%$ of all had positive results (271 fem, 146 male). 356 showed allo- and/or auto-antibodies (141 pre-known, 215 newly diagnosed). 145 had a single antibody and 211 cases had multiple antibodies (table). 62 samples had a non-specific direct agglutination test (ab-screening / elution negative).

\begin{tabular}{|c|c|c|c|c|c|c|}
\hline Antibody & Total & $\begin{array}{l}\text { Singular } \\
\text { antibodies }\end{array}$ & $\begin{array}{l}\text { Antibodies in } \\
\text { combination }\end{array}$ & $\begin{array}{l}\text { Additional } \\
\text { warm auto- } \\
\text { antibodies }\end{array}$ & $\begin{array}{l}\text { Newly } \\
\text { identified }\end{array}$ & $\begin{array}{l}\text { Pre-known } \\
\text { antibodies }\end{array}$ \\
\hline Anti-D & $\begin{array}{l}47(8 \times \mathrm{Rh}- \\
\text { prophylaxis })\end{array}$ & 27 & 20 & & 26 & 21 \\
\hline Anti-E & 76 & 24 & 52 & 8 & 24 & 5 \\
\hline Anti-C & 29 & 3 & $\begin{array}{l}26(12 \times+ \\
\text { Anti-D, } 2 \times+ \\
\text { Anti-D/-E })\end{array}$ & 3 & 7 & 21 \\
\hline Anti-c & 26 & 9 & 17 & - & 5 & 21 \\
\hline Anti-e & $\begin{array}{l}15(14 \times \\
\text { Auto-Anti-e) }\end{array}$ & 1 & - & 14 & 1 & 0 \\
\hline \begin{tabular}{|l|} 
Anti-Cw \\
\end{tabular} & 15 & 5 & 10 & & 6 & 9 \\
\hline Anti-K & 49 & 20 & 29 & - & 15 & 34 \\
\hline \begin{tabular}{|l} 
Anti-Jk \\
a
\end{tabular} & 19 & 10 & 9 & 2 & 8 & 11 \\
\hline Anti-Jk $^{\mathrm{b}}$ & 9 & 3 & 6 & - & 2 & 7 \\
\hline Anti-Fy $^{a}$ & 21 & 12 & $\begin{array}{l}9(2 \times+ \\
\text { HTLA })\end{array}$ & - & 10 & 11 \\
\hline Anti-Fy $^{b}$ & 2 & - & 2 & - & 1 & 1 \\
\hline Anti-M & 15 & 13 & 2 & - & 13 & 2 \\
\hline Anti-S & 10 & - & $\begin{array}{l}10(3 \times+ \\
\text { Anti-Wra })\end{array}$ & 3 & 1 & 9 \\
\hline \begin{tabular}{|l} 
Anti-Wr \\
a
\end{tabular} & 47 & 8 & $\begin{array}{l}39(14 \times \\
+ \text { WAA })\end{array}$ & 14 & 6 & 41 \\
\hline Anti-Lu ${ }^{\mathrm{a}}$ & 26 & 20 & 6 & - & 3 & 23 \\
\hline
\end{tabular}

+ Rare antibodies: $1 \times$ Anti-k, Anti-Ch ${ }^{\mathrm{a}}$, Anti-Cs ${ }^{\mathrm{a}}$, Anti-Kn ${ }^{\mathrm{a}}$, Anti-Yta ${ }^{\mathrm{a}}$ HLA-Antibodies, 2x Anti-Kn ${ }^{\mathrm{a}}$
Conclusion: The fact that $14.1 \%$ of all samples ( $39.6 \%$ of all positive) contained previously identified antibodies, most of them only by chance known to the reference lab, but not to clinic shows deficiencies in the communication of relevant immunohematological results. The probability of existing RBC antibodies increases with age $30 \%$ of antibody cases: year of birth 1930-1939)and with complex transfusion history but the availability of known data is currently inadequate. It is questionable whether the established system with the issuance of emergency cards is keeping with the times or should be replaced by e.g. electronical media.

\section{IMH-P39 \\ eBlood Match System - Telemedicine in Pre-transfusion Blood Match Tests}

\section{J. Tasič ${ }^{1},{ }^{*} U$. Lozar ${ }^{2}$ \\ ${ }^{1}$ Universität, Faculty for Electrical Engineering, Ljubljana, Slovenia ${ }^{2}$ PPS , Ljubljana, Slovenia}

Background: Blood transfusion services are usually organized as a network. Blood products are delivered from a central blood bank to the user, the local hospital. Before a blood transfusion is given to a patient, obligatory pre transfusion tests are performed at the hospital laboratory to confirm donor-patient compatibility. These tests are normally performed by specially trained personnel. Not all local hospitals have trained personnel available $24 / 7$ to perform such tests. The idea is to concentrate knowledge in the reference laboratories and use telemedicine to support local hospitals.

Methods: We have developed a telemedicine system for blood transfusion work to supply the local hospital laboratories with an expert opinion from the central reference laboratory. The eBlood Match System allows remote inspection and interpretation of pre-transfusion tests, which are performed by micro tube gel cards. Gel card reader scans the picture of a gel card and the operator in local hospital sends it via internet to the specialist in the reference laboratory. Scanned picture with patient's historical data are available to the specialist, when he/she does the interpretation. Final result is transmitted back to the hospital's laboratory. Digital signature and secure data transmission assure traceability and safety. Typical client-server database structure supports all functionalities, including videoconference, SMS alerts, digital signature, archiving, etc.

Results: The system was installed in all blood transfusion laboratories inSlovenia. Until now over 100.000 interpretations were performed. The same quality of the service was assured for all laboratories countrywide. Intelligent software module supervises the interpretation performed by specialist and warns in case of ambiguity. Main features:

- capture of high-resolution images of gel cards

- magnification of the images for detailed observation of agglutinates

- 24/7 availability of expertise

- real-time interaction between the technician in the local hospital laboratory and the expert in the reference laboratory

- exchange of medical data for patients and blood donors for the provision of information about transfusions and historical cases

- complete traceability of all procedures

- reliable, safe, secure and encrypted data transmissions

- compliance with EU legislation

Conclusion: Validation of the eBlood Match system was performed using 99 clinical cases selected from daily work. Two groups of specialists participated. Group A performed the readout of the pretransfusion tests using the telemedicine system. Group B read the ID cards independently using the standard visual method. All 98 final interpretations recorded using the telemedicine system were correct. We recorded 591 micro-tube readouts of agglutination strength using the telemedicine system, of which 582 were correct. Also 582 were correct using standard visual method. The validation proved that the telemedicine system was suitable for operational use. 

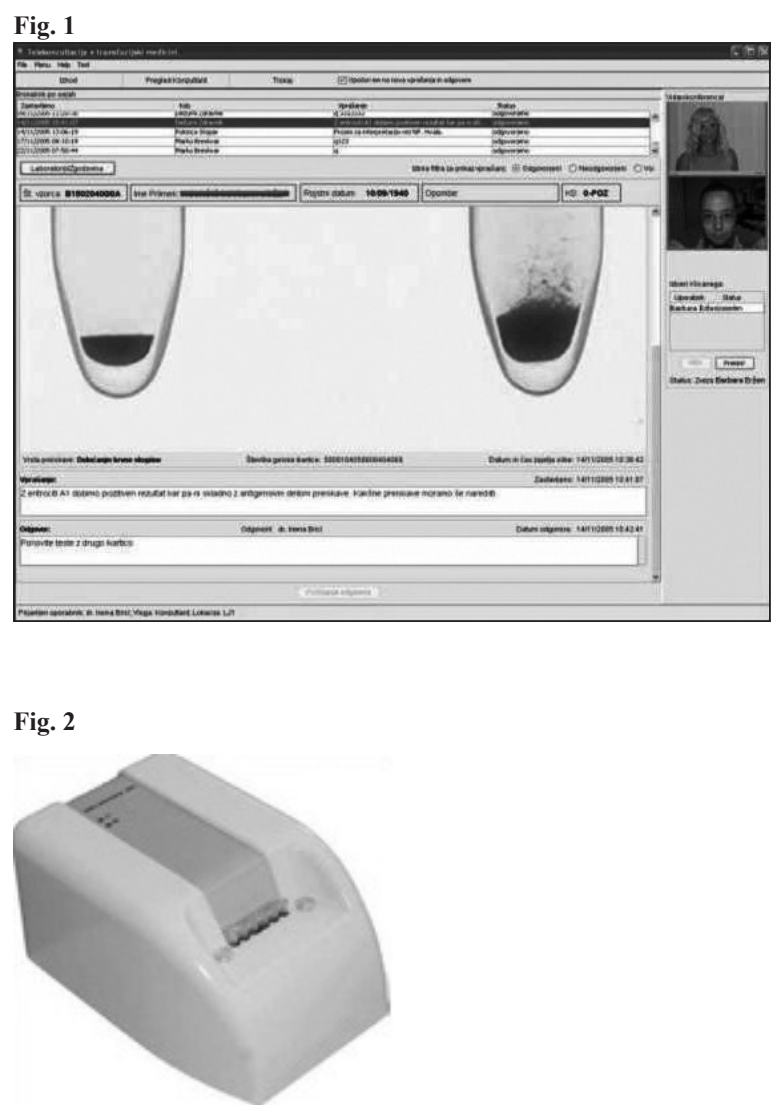

IMH-P40

Immuno-modulatory properties of primary-like immortalized stroma cell lines from leukemia patients

${ }^{*}$ B. Wagner', J.R. Göthert', U. Dührsen², T. May³, P.A. Horn ${ }^{1}$, B. Opalka', V. Rebmann ${ }^{1}$

${ }^{1}$ Uniklinikum Essen, Transfusionsmedizin, Essen, Germany

${ }^{2}$ Uniklinikum Essen, Hämatologie, Essen, Germany

${ }^{3}$ InSCREENeX GmbH, Braunschweig, Germany

Background: In leukemia, and acute myeloid leukemia (AML) in particular, cross-talk between the malignant cells and stromal cells in the bone marrow (BM) microenvironment is considered to play a role in blocking of cell death, stimulation of proliferation, drug resistance induction, and immune suppression.

Methods: To study such stromal effects in more detail and with an unlimited source of cells we had established three BM stroma cell lines from samples of two AML patients (K-AML and CZ-AML) and a sample from a normal donor (N-KM). The technique used for immortalization is considered to maintain the phenotype of the primary cells as much as possible. The cells reveal a common mesenchymal stem cell surface marker profile and have been in continuous culture for $>1$ year. Here, we studied the immunomodulatory properties of soluble factors derived from these cell lines in comparison to four AML derived primary stroma cells on the induction of regulatory $\mathrm{T}$ cells. After the incubation of peripheral blood mononuclear cells (PBMCs) in presence and absence of BM stroma cell line's supernatant during 7 days, the CD4 positive cells were analyzed by flow cytometry and quantitative Real-time PCR.

Results: We did not observe a change of the percentage of CD4+CD25+FOXP3 + cells in the flow cytometric analysis. In contrast, an 1.5-fold increase of FOXP3 mean fluorescence intensity (MFI) was found for CD4 T cells cultured with supernatant of the K-AML cell line compared to CZ-AML and normal donor. Culturing PBMCs with supernatant of AML derived primary stroma cells from four different AML patients revealed for two supernatants an induction of FOXP3 comparable to the
K-AML supernatant, whereas the other two primary AML stroma supernatants showed no effect on the FOXP3 expression level. These results were verified by quantitative FOXP3 specific Real-Time PCR. Moreover, the biophysical characterization by the Amnis Image Stream instrument showed that the CD4+CD25high+FOXP3+ cells seem to be smaller than the CD4+CD25low+FOXP3+ cells.

Conclusion: The supernatant of the two novel BM stroma cell lines derived from AML patients showed a converse behavior in case of the up-regulation of FOXP3 in CD4+CD25+ T cells, which was confirmed by comparison of AML-derived primary stroma cell supernatants. Thus, it seems that these cell lines are qualified to investigate immune modulatory properties of soluble factors being part of a leukemic BM microenvironment, e.g. modulation of immune cells.

This work was partly supported by Deutsche Krebshilfe (grant no. 109816).

\section{IMH-P41 \\ Mirtazapine-induced immune thrombocytopenia in a patient with preexisting thrombocytopenia}

*K. Winterstein, R. Danneberg, A. Büttner, H. Kroll

Red Cross Blood Transfusion Service NSTOB, Institute for Transfusion Medicine Dessau, Dessau, Germany

Background: Purpose: Drug-induced immune hemocytopenias are rare but potentially severe side effects of several drugs. Detection of the responsible antibodies may prevent the patient from reexposition to the drug.

Methods: Case report: A 50-year-old male patient suffering from ethyl toxic liver cirrhosis Child-Pugh-C with bleeding disorder and intracranial hemorrhage received a daily dose of mirtazapine because of repeated depressive episodes. Further medications were lorazepam, propranolol, pantoprazole, levetiracetam, haloperidol and metamizol. The platelet count was decreased $(85,000 / \mu \mathrm{L}-103.000 / \mu 1)$ when starting treatment. Undergoing mirtazapine therapy the platelet count dropped to $42,000 / \mu \mathrm{L}$. Three days later platelet count of $33,000 / \mu \mathrm{L}$ was measured. Serologic examination was initiated because immune thrombocytopenia was suspected. After discontinuation of mirtazapine the platelet count rose immediately to $154,000 / \mu \mathrm{L}$ during the following days.

Methods: The serum was analysed by platelet suspension immunofluorescence test (PSIFT) and glycoprotein-specific immunoassay (MAIPA). Results: Upon flow cytometric PSIFT the serum was strongly positive in the presence of $1 \mathrm{mg} / \mathrm{mL}$ mirtazapine but negative in the absence of the drug (MFI ratio: 24) indicating mirtazapine-dependent platelet-reactive antibodies. No reactivity was detectable in the presence of lorazepam, propranolol, pantoprazole, levetiracetam, haloperidol and metamizol. No binding was detected on platelet glycoproteins IIbIIIa, IbIX, V or IaIIa in MAIPA assay in the absence of the drug.

Conclusion: We describe a further case with mirtazapine-induced thrombocytopenia. This drug seems to bear a special risk for the induction of drug-dependent antibodies. Mirtazapine is that antidepressive drug with second highest prescription in germany. Physicians who use mirtazapine should be aware of this side effect.

\section{IMH-P42}

\section{Experience with heat elution in a regional blood bank}

\section{${ }^{*}$ T. Lorenzen, G. Walther-Wenke}

DRK-Blutspendedienst West, Zentrum für Transfusionsmedizin, Münster, Germany

Background: Heat elution is one of the preferred techniques for the investigation of patient samples with positive direct antiglobulin test (pdat) and transfusions within the last 6 weeks. A number of such samples reach the laboratory outside normal working hours. The question arises whether this time consuming analysis improves transfusion safety especially at night and on weekends. 
Methods: A retrospective analysis of test results of all cases with heat elution in 2011 was performed.

Results: Investigations were performed on 540 patient samples. In total 52 eluates reacted positive in antibody screening. In 49 cases further investigations showed no diagnostic benefit. Just in 3 cases specific antibodies were detected in the eluate only. In one case auto-anti- $\mathrm{C}^{\mathrm{w}}$ and auto-anti-E were identified, in a $2^{\text {nd }}$ case anti- $\mathrm{C}^{\mathrm{w}}$ was found in addition to previously known anti-E and anti-C. In the $3^{\text {rd }}$ case auto-anti-C was identified in addition to anti-E in the patient's plasma.

Conclusion: Published data show comparable numbers of positive results with elution. Only 3 of the 52 reacting eluates in 540 patient samples revealed additional antibodies in the eluate. Elution should not postpone transfusions unnecessarily. Especially when posting samples to an reference laboratory, time consuming investigations and transports add further to the time delay. Performing elutions on samples with pdat is of limited value.

\section{IMH-P43}

\section{A case of hemolytic disease of the fetus (HDFN) due to anti-D and anti-G probably masquerading as anti-C}

\author{
${ }^{*}$ K. Rosskopf, H. Knausz, G. Lanzer, B. Csapo, T. Wagner \\ Univ. Klinikum Graz, Univ. KI. f. Blutgruppenserologie und \\ Transfusionsmedizin, Graz, Austria
}

Background: Antibodies with apparent anti-C plus anti-D specificity could shape up as anti-C plus anti-D plus anti-G or as anti-C plus anti-G or as anti-D plus anti-G or as Anti-G only. Clinical significance is present when an anti-D prophylaxis is avoided whereas no immunized anti-D is proven or the apparent immunization of an antibody is doubted due to lack of contact to the corresponding antigen.

Methods: A woman in sixth pregnancy, week 31 of gestation, with B Rh negative ccddee who had two abortions and one fetal death in her medical history had anti-D titer 1:512 and anti-C titer 1:64 and anti-E below detection limit (anti-E was specified earlier in the same pregnancy). The fetus was tested D positive by maternal plasma nucleic acid testing. The fetus needed intrauterine transfusion due to ultra sound signs of anemia in our university hospital. Her husband has blood group O Rh ccD.EE and doubts raised about the specificity of her antibodies. A sequential adsorption-elution using the woman's serum and test cells of ccD.EE (C-, $\left.\mathrm{D}^{+}, \mathrm{G}^{+}\right)$and Ccddee $\left(\mathrm{C}+, \mathrm{D}-, \mathrm{G}^{+}\right)$specificity was done. In addition the supernatants after adsorption using the same test cells were analysed for presence of Anti-C and Anti-D antibodies, respectively.

Results: The sequential adsorption-elution study confirmed the presence of Anti-G antibody. After incubation with ccD.EE cells anti-C, anti-D and Anti-G antibodies were found in the supernatant. So the proof that there was no Anti-C in the woman's serum failed. The testing of the supernatant after incubation with Ccddee cells confirmed the presence of Anti-D and Anti-G antibodies.

Conclusion: We present a case of a pregnant woman with apparent Anti-CD antibody composition. The fetus tested D positive showed signs of HDFN. Therefore intrauterine transfusion was given. By adsorption and elution procedures the antibody specification of the woman was adjusted from anti-C plus anti-D to anti-D plus anti-G. Anti-C could neither be confirmed nor excluded. The woman gave birth to a baby in week 33 with apparent blood group $\mathrm{O}$ negative due to intrauterine transfusion.

\section{Oral Abstract Session: Infektionssicherheit}

\author{
INF-V01
}

\section{Trends in infections among blood donors in Germany -} are we still on the safe side?

\section{${ }^{*}$ R. Offergeld, M. Askar, S. Ritter, O. Hamouda \\ Robert Koch-Institut, Abteilung für Infektionsepidemiologie, Berlin, Germany}

Background: The Robert Koch Institute is responsible for the nationwide surveillance of HIV, hepatitis C (HCV), hepatitis B (HBV) and syphilis infections among blood and plasma donors in Germany.

Methods: All blood establishments in Germany are requested to report aggregated data on the number of donors and donations tested for infections together with detailed epidemiological and serological information on confirmed positive donors. Infection rates stratified for age group, gender, type of donation and donor type can thus be calculated.

Differences between donor groups were assessed with a Poisson regression.

Results: In 2011 a total of 542,542 new and applicant donors (ND) and $7,028,062$ donations from 2,523,769 repeat donors (RD) were reported. Among all donors 1481 confirmed HIV, HCV, HBV and syphilis infections were diagnosed. The resulting prevalence per 100,000 ND was 7.4 for HIV, 61.9 for HCV, 116.9 for HBV and 41.4 for syphilis. The proportion of incident infections $/ 100,000$ donations from RD was 1.0 for HIV, 0.7 for HCV, 0.4 for HBV and 1.5 for syphilis. Male donors were significantly more affected by transfusion relevant infections than female donors, especially with respect to HIV and HBV infections. While the prevalence increased with donor age, the highest rate of incident infections occurred in the donor group aged 25 to 34 .

19 confirmed NAT-only infections were reported (7 HIV, $9 \mathrm{HCV}$ and $3 \mathrm{HBV}$ infections), all of these were found in RD and the majority in male donors (all HIV and HBV NAT-only infections and 6/9 HCV-NAT-only infections). For 20,295 donors a confidential unit exclusion (CUE) was reported resulting in a loss of $0.36 \%$ of donations in the corresponding blood establishments. 11 of these donors were found to be confirmed positive for at least one of the infections. Since 2001 the prevalence and incidence of HCV and HBV among blood donors has declined considerably. By contrast, the incidence of HIV infections shows an increasing trend. Within 10 years the rate of HIV infections among RD nearly doubled, while HCV and HBV infection rates decreased by 59 and $74 \%$, respectively.

Conclusion: The continuous increase in HIV infection rates in repeat donors might compromise transfusion safety. To counteract the present non-adherence with donor deferral criteria and to enhance the effectiveness of the CUE, improved donor questionnaires, CUE forms and educational material are needed and HIV-test-seeking should be addressed directly.

\section{INF-V02 \\ New HIV-1 variants missed during routine blood donor NAT-screening}

${ }^{*}$ B. Müller' ${ }^{1}$ M. Nübling ${ }^{2}$, J. Kress ${ }^{2}$, K. Roth ${ }^{3}$, S. De Zolt ${ }^{3}$, L. Pichl'

'DRK blutspendedienst West, NAT-Labor, Hagen, Germany

${ }^{2}$ Paul-Ehrlich Institut, Molekulare Virologie, Langen, Germany

${ }^{3}$ GFE Blut mbH, Frankfurt, Germany

Background: Nucleic acid amplification techniques (NAT) in routine blood donor screening (RBDS) considerably reduce the diagnostic window phase period. Nevertheless, several reports of false-negative NAT results due to different reasons were published. Here, four cases of HIV-1 RNA-positive blood donations that escaped detection by NAT-screening are described. 
Methods: About 3 million blood donations were screened for viral infections between January 2010 and October 2012 in our German Red Cross blood donation service. Four plasma specimens with false-negative HIV-1 NAT results were comparatively investigated with 12 CE-marked NAT assays. In two cases of putative HIV-1 variants the target region of the NAT assay was sequenced allowing comparison with the respective primers and probes.

Results: Most of the NAT assays used in RBDS with the 5'-LTR as target region demonstrated deficiencies in detecting the viral variants and the low viral carrier donations. In one case several primer mismatches due to point mutations in the target region were observed. In contrast, sequence analysis revealed in another case a deletion of 56 nucleotides within the 5'-LTR preventing the binding of the probe accompanied by a neighbored insertion of another 52 nucleotides. Using dual-target assays, no false-negative results were obtained for these cases. The remaining two false-negative test results arose from the viral load of the specimen, which was below the respective NAT's LOD.

Conclusion: HIV-1 is characterized by a high mutation rate and rapid generation of new viral variants. By the use of only one target region for HIV-1 NAT assays there is a certain risk of false-negative test results. Employing of HIV-1 multi-/dual-target assays in RBDS seems to be a reasonable and cost-effective possibility to minimize this problem.

\section{INF-V03 \\ Incidence of hepatitis E virus infections in blood donors from Hessia}

\section{${ }^{*}$ K. Hourfar, W. Sireis, M. Schmidt, E. Seifried}

DRK Blutspendedienst Baden-Württemberg - Hessen, Institut Frankfurt am Main, Spenderscreening, Frankfurt am Main, Germany

Background: Several transfusion transmitted hepatitis E infections have been reported, although the major infection pathway is via the fecal oral route. Previous studies from UK, Germany, Sweden and Japan demonstrated prevalence in a range between 1 in 4,000 and 1 in 10,000.

Methods: The current study reports on the incidence of HEV RNA positive donations in blood donors from hessia. A first study was already conducted in 2012 at our blood donor service and demonstrated an incidence of HEV RNA positive donations of approximately 1:3000. This second study was carried out in order to determine the incidence with higher statistical power and to identify seasonal and geographical differences.

A PCR assay for HEV-RNA was used to screen blood donations from hessia between January and March 2013. The assay was used in combination of our automated NAT platform Zelos x100. To identify the incidence of Hepatitis E-virus infections in our blood donor population the NAT assay was used on pools of up to 94 donations per pool.

Results: The combination of our extraction system which is based on a high volume magnetic particle extraction process and the PCR assay allows highly sensitive detection of Hepatitis E-Virus. The $95 \%$ level of detection on basis of the WHO/PEI standard preparation was $11,3 \mathrm{IU} / \mathrm{ml}$ as calculated by probit analysis.

From January to March 2013 a total of 54,780 donations in 597 pools were screened for HEV-RNA. From these pools 34 were found to be HEV-RNA positive. This corresponds to an incidence of HEV RNA positive donations of approximately 1 in 1600 and is therefore even higher that expected. Analysis of the geographical distribution is in process.

Conclusion: Compared to the incidence of HEV infections in blood donors the reported cases of transfusion transmittet HEV is very low. However screening for HEV might be required for certain plasma products. The high incidence of HEV-RNA would cause a logistical challenge for minipool NAT testing due to the need for frequent pool resolution.

\section{INF-V04 \\ Strategies for hepatitis E virus blood donor screening: Comparison of four NAT assays versus antigen testing}

\section{${ }^{*}$ T. Vollmer, C. Knabbe, J. Dreier}

Herz- und Diabeteszentrum NRW, Insitut für Laboratoriums- und Transfusionsmedizin, Bad Oeynhausen, Germany

Background: The risk of transfusion-transmitted HEV infections by contaminated blood products currently remains unknown, amongst others due to the absence of available NAT screening methods. The sensitivity and performance of different HEV RNA amplification systems were evaluated for (a) blood donor pool screening and, (b) individuals with acute or chronic infections. HEV antigen screening was evaluated in comparison to NAT screening and detection of HEV-IgM specific antibodies using seroconversion panels of ten virologically confirmed HEV genotype 3 infected individuals.

Methods: The analytical sensitivity and the precision of the 3 commercially available assays RealStar HEV RT-PCR assay (Altona Diagnostic Technologies), hepatitis@ceeramTools Kit (Ceeram), the ampliCube HEV RT-PCR Kit (Mikrogen) and one in-house RT-PCR assay was determined using a twofold dilution series of plasma inoculated with the first WHO hepatitis E virus RNA standard (Paul-Ehrlich institute) in 6 dilution steps and 24 replicates. RNA was extracted with a high volume extraction protocol (4.8 ml, chemagic Viral 5K, Perkin Elmer) for blood donor pool screening and the Nuclisens easyMAG (biomerieux) for IDNAT. Screening for HEV antigen in consecutive samples of ten HEVRNA positive donors was performed using an HEV antigen test (Axiom) and the presence of HEV-specific IgM antibodies was determined using the recomWell HEV-IgM immunoassay (Mikrogen).

Results: All assays provided a good analytical sensitivity ranging from 4.7 to $91.2 \mathrm{IU} / \mathrm{ml}$ (96 pool: $451-8755 \mathrm{IU} / \mathrm{ml}$ per individual donation) using the high volume extraction method. The analytical sensitivities observed for the ID-NAT ranged from 37.8 to $180.1 \mathrm{IU} / \mathrm{ml}$. All assays demonstrate intra- and interassay variabilities with variation coefficients $<3 \%$. HEV antigen was only detectable in four out of ten samples, with corresponding viremia ranging from $1.92 \mathrm{E}+03$ to $2.19 \mathrm{E}+05 \mathrm{IU} / \mathrm{mL}$. The progression of $\mathrm{HEV}$ antigen detection follows the progression of $\mathrm{HEV}$ viremia, for all these four donors. HEV viremias in antigen-negative donors ranged from $1.86 \mathrm{E}+01$ up to $4.74 \mathrm{E}+04 \mathrm{IU} / \mathrm{mL}$. HEV-specific IgM antibodies were detectable in samples of seven donors, only one donor presented a parallel positivity of HEV antigen and anti-HEV IgM antibodies. Conclusion: The clinical relevance of transfusion-associated hepatitis $\mathrm{E}$ infection still requires further investigations. In case of raising concerns regarding blood product safety, the evaluated methods present powerful basic tools providing sensitive possibilities for viral testing comprehending further investigative studies. Application of HEV antigen screening is not an option to detect current HEV infection due the decreased sensitivity compared to NAT methods.

\section{INF-V05}

Risk-assessment of transfusion-associated Babesiosis in Tyrol

${ }^{*}$ S. T. Sonnleitner ${ }^{1}$, R. Baumgartner ${ }^{1}$, J. Simeoni ${ }^{1,2}$, H. Schennach ${ }^{3}$, C. Lass-Flörl ${ }^{3,4}$, R. Edelhofer ${ }^{5}$, K. Pfister ${ }^{6}$, G. Walder

${ }^{1}$ Dr. Gernot Walder $\mathrm{GmbH}$, Außervillgraten, Austria

2Service for Hygiene and Public Health, Bozen, Italy

${ }^{3}$ University Clinics Innsbruck, Central Institute for Blood Transfusion and Immunology, Innsbruck, Austria

${ }^{4}$ Innsbruck Medical University, Department of Hygiene, Medical

Microbiology and Social Medicine, Innbruck, Austria

${ }^{5}$ University of Veterinary Medicine, Department of pathophylsiology,

Vienna, Austria

${ }^{6}$ Veterinary Faculty, Comparative Tropical Medicine and Parasitology, Munich, Germany

Background: After malaria, babesiosis is the second most common transfusion-transmitted parasitic disease in the USA. This study shows that Babesia spp. is present in the Tyrols, that blood donors come into im- 
munoreactive contact with it and that we have to consider how to sustain blood product safety concerning this new challenge.

Babesiosis recently gained in importance as an emerging zoonotic infection in humans. Thereby, $B$. microti, $B$. divergens, $B$. venatorum and $B$. duncani are the four species of Babesia that are generally able to infect people.

Besides transmission of Babesia spp. by ixodid ticks, also transmission through blood transfusion has to be considered.

Methods: The area of investigation comprises the Austrian region of the Tyrols with North and East Tyrol with the rivers Drau (East Tyrol), Inn and Lech (North Tyrol). A number of 988 sera were tested for IgG antibodies against $B$. divergens and $B$. microti by in-house immunofluorescence assays (IFA). IFA-slides were tested by using commercially available hyperimmunesera.

Collection of questing ticks was performed in summer 2009 by about 120 volunteers among hunters at 25 sampling sites over a period of three months by flagging. Compared data of seroprevalence were tested by a chi-squared test. Differences were considered statistically significant when $\mathrm{x}^{2}>3.841(P$ was $\leq 0.05)$

Results: Of 988 sera, $21(2.1 \%)$ were positive in IFA against the $B$. divergens-complex at titres of $1: 128$ or higher and $5(0.5 \%)$ were positive in IFA against $B$. microti. The confidence interval for a sample size of 988 lies at $0.4 \%$.

Not one serum reacted against both Babesia-species, indicating that cross-reactivity is lower than $19.1 \%$ between $B$. divergens and $B$. microti $(p \leq 0.05)$ and specifity lies at $80.9 \%$.

Calculation of the risk-assessment:

We investigated 988 Tyrolean blood donors with an average age of 39.3, resulting in 38,840 years of life. Among them we found 21 seropositives against $B$. divergens. Under the presumption of a long-lasting immune response we can expect $0.5( \pm 0.2,95 \%)$ seroconversions against $B$. divergens per 10.000 persons per year. For $B$. microti the same calculation results in $0.1( \pm 0.08,95 \%)$ seroconversions per 10.000 persons per year. Conclusion: The present study for the first time assesses concomitant seroepidemiology to both $B$. divergens and $B$. microti. It shows that the local population comes into seroreactive contact with at least one member of the B. divergens-complex and - to a lesser extent - B. microti. Generally, it is one of only a few seroepidemiologic studies on Babesia spp. in European blood donors. The possibility of the asymptomatic persistence of Babesia spp. in blood is supported by numerous cases. A similar phenomenon of persistent and relapsing infection with Babesia spp. was observed by our own work group concerning a 3 -year-old child from North Tyrol.

\section{INF-V06 \\ Influence of bacterial growth properties in PCs on bacterial platelet screening strategies}

\section{${ }^{*}$ M. Störmer' ${ }^{1}$ S. Radojska1, V.-M. Petrescu-Jipa' , N.J. Hos², B. Gathof'}

${ }^{1}$ Transfusion Medicine, University Hospital of Cologne, Cologne, Germany ${ }^{2}$ Medical Microbiology, Immunology and Hygiene, University Hospital of Cologne, Cologne, Germany

Background: Since screening for bacterial contamination has been recommended by the Paul-Ehrlich-Institut for prolonging the shelf-life of platelet concentrates (PCs) back to 5 days in Germany, a variety of bacterial detection strategies were implemented. But the applicability and benefit of these methods is always influenced by the bacterial growth properties in the product. This study demonstrates the influence of bacterial growth ability on direct and indirect bacterial detection in PCs.

Methods: K. pneumoniae; E. coli, S. pyogenes, S. epidermidis, and B. thuringiensis were inoculated separately with 5-90 CFU/bag into PCs and incubated under routine conditions. Platelet function analysis of freshly produced apheresis PCs (APCs) was performed by using the Multiplate Analyzer (Roche Diagnostics) and aggregometry according to Born. The BactiFlow ALS (bioMérieux AES Chemunex) was used for direct detection and enumeration of bacteria in fresh and expired pooled and apheresis PCs. Sterile PCs served as negative controls. All measurements were performed in parallel to culture as reference. The identity of isolated bacteria was proven by MALDI-TOF analysis.

Results: $B$. thuringiensis and $K$. pneumoniae grew rapidly to high counts in all contaminated products. E. coli and $S$. pyogenes showed both, postponed and rapid growth behaviour. $S$. epidermidis grew slowly and was detectable by using the BactiFlow in $50 \%$ on day 4 (100\% on day 7$)$. All other strains were detectable on day 2 in $92 \%$ (100\% on day 3$)$. BactiFlow results corresponded to the results obtained from culture and plate assay. Platelet aggregation was reduced in highly bacterially contaminated units. The level of reduction was dependent on donors and bacterial strain growth properties. Rapid growers resulted in a reduction of platelet aggregation already on day 2 . Intermediate growing organisms were detectable on day 3 while slow growers resulted in a reduced aggregation upon day 3 or 4. S. epidermidis showed only an influence on aggregation when reaching high bacterial count levels on day 7 .

Conclusion: Bacterial detection in PCs is reliant on strain growth properties. Direct detection of bacterial cells have the benefit to detect bacteria in better time and represents the current situation of contamination. In contrast, indirect detection represents only the situation of a specific time before measurement due to the time between reaching high bacterial cell counts and inducing the detectable effect. Measuring platelet aggregation beforen transfusion would not only provide a lower priced alternative but could also improve microbiological safety, quality and efficiency of platelet transfusion.

\section{INF-V07 \\ UVC-treatment of residual leukocytes in platelet concentrates prevents XGVHD development in a NOD SCID gamma (NSG) mice model}

${ }^{*}$ P. Pohler ${ }^{1}$, M. Müller ${ }^{2}$, D. Schaudien², K. Sewald², T. H. Müller ${ }^{3}$, A. Seltsam

${ }^{1}$ DRK-Blutspendedienst NSTOB, Forschung und Entwicklung, Springe, Germany

${ }^{2}$ Fraunhofer Institute of Toxicology and Experimental Medicine, Hannover, Germany

${ }^{3}$ DRK-Blutspendedienst NSTOB, Springe, Germany

Background: One of the deleterious effects of leukocytes present in blood components is the transfusion-associated graft versus host disease (TA-GvHD). It is mediated by lymphocytes in donated blood, which proliferate and mount a severe immunological response in susceptible patients. The THERAFLEX UV-Platelets system (Macopharma) uses UVC light for pathogen reduction. Previous in vitro studies have shown that UVC-irradiation inactivated T-cells by $>5 \log$ steps. In the present study, we used a xenogeneic mouse model to evaluate the efficacy of UVC to prevent GvHD in vivo.

Methods: Plasma-reduced PCs prepared from 5 buffy coats in additive solution $\mathrm{SSP}^{+}$were spiked with 1 x $10^{6} / \mathrm{mL}$ human peripheral blood mononuclear cells (PBMCs) and treated either with UVC (standard dose of 0.2 $\mathrm{J} / \mathrm{cm}^{2}$ ), $\gamma$-irradiation (30 Gy) or remained untreated (positive control). PB$\mathrm{MCs}$ were removed from PCs and transferred into nod scid gamma mice (2.0-2.5 x 107 PBMCs/mouse). A total of 12 mice per study arm were treated with PBMCs. GvHD development was observed over a period of 61 days. Animal health and GvHD development were monitored by changes in external behaviour and phenotype, weight loss, survival days, and engraftment of human cells in different organs of the recipient mice by flow cytometry and immunohistological staining using human PBMC-specific antibodies (e.g. CD45, CD3, CD4, CD8, and CD16/CD56). In addition, a comprehensive histopathological evaluation was performed on all mice. Results: All mice receiving untreated human PBMCs (control group) exhibited severe symptoms of GvHD with weight loss, ruffled fur and reduced reactivity within 25 days after injection. High numbers of human T-cells and NK-cells were detected in various tissues like spleen, blood and bone marrow, but also in the skin, lung liver and gastro intestinal tract. Major histopathological findings were human cell infiltration ac- 
companied by a decreased cellularity of the bone marrow and a depletion of the red pulp of the spleen. In contrast, proliferation of human cell and development of GvHD was not detectable in animals inoculated with UVC-treated or gamma-irradiated PBMCs. Interestingly, human lymphocytes were observed in 2 out of 12 mice in the gamma group, whereas in the UVC group no human lymphocytes were detectable.

Conclusion: UVC irradiation effectively eliminates the proliferative capacity of residual PBMCs in PCs and inhibits the development of GvHD in a mouse model. These results indicate that the UVC-based THERAFLEX UV-Platelets system has the potential to substitute gamma irradiation for TA-GvHD prevention.

\section{INF-V08}

\section{Open Phase I Clinical Trial on Safety and Tolerance of autologous UVC-treated Single Donor Platelet Concentrates in Healthy Volunteers - Dose Escalation Study}

\section{${ }^{*}$ T. Thiele ${ }^{1}$, P. Pohler 2 , T. Kohlmann 3 , A. Sümnig' 1 K. Aurich ${ }^{1}$,} K. Selleng ${ }^{1}$, A. Westphal ${ }^{1}$, T. Müller ${ }^{2}$, A. Seltsam², A. Greinacher ${ }^{1}$

${ }^{1}$ Universitätsmedizin Greifswald, Transfusionsmedizin, Greifswald, Germany ${ }^{2}$ DRK NSTOB , Springe, Germany

${ }^{3}$ Universitätsmedizin Greifswald, Community Medicine, Greifswald, Germany

Background: UVC-pathogen reduction for platelet concentrates (PCs) is the first available procedure based on UVC-light alone without addition of photo additives. We investigated the safety and tolerance of UVC-pathogen reduction in recipients of autologous UVC-treated PCs. Methods: Healthy volunteers $(n=11)$ eligible for double platelet apheresis underwent two single (series $1+2$ ) and one double apheresis procedures (series 3 ) with a target yield of $3 \times 10^{11}$ platelets per PC. PCs were treated with UVC light $\left(0.2 \mathrm{~J} / \mathrm{cm}^{2}\right)$ and retransfused after two days storage in a dose-escalation scheme. Heart rate, temperature and blood pressure were determined along with primary endpoints fibrinogen, aPTT, PT (Quick) and D-Dimer before and after each transfusion. Secondary endpoints were $1 \mathrm{~h}$ corrected count increments (CCI) after each PC-transfusion in series 2 and 3 and standard clinical chemistry (electrolytes, liver enzymes, creatinine, CRP etc.).

Results: Heart rate, temperature and blood pressure were not relevantly changed. For primary endpoints, no significant changes were observed before and after transfusion of UVC-treated PCs. The median and interquartile range of 1-h CCIs were 16.0 (range: 12.0-22.0)after transfusion of one PC in series 2 and 13.0 (range: 7.8-26.5) after transfusion of each PC in series 3. Conclusion: Transfusion of UVC-treated autologous PCs met safety and tolerance criteria in healthy volunteers. The CCIs, albeit experimental in the autologous setting, suggest a good viability of transfused UVC-treated platelets. The results of this study justify as a next step of clinical evaluation the conduction of a study in transfusion-dependent patients. EudraCT No. 2010-023404-26.

\section{Poster: Infektionssicherheit}

\section{INF-P01}

In vitro quality of platelets treated in the THERAFLEX UVPlatelets system is well preserved during storage

\section{*U. Gravemann ${ }^{1}$, P. Pohler ${ }^{1}$, T.H. Müller², A. Seltsam ${ }^{1}$}

${ }^{1}$ DRK-Blutspendedienst NSTOB, Forschung und Entwicklung, Springe, Germany

${ }^{2}$ DRK-Blutspendedienst NSTOB, Springe, Germany

Background: The THERAFLEX UV-Platelets system (Macopharma) uses UVC light (wavelength: $254 \mathrm{~nm}$ ) for pathogen reduction of PCs without the need of any additional photoactive compound. UVC treatment selectively affects the nucleic acid of pathogens as well as of leu- kocytes while proteins are largely preserved. In the current study, the accumulation of cytokines during storage was investigated. The ThromboLUX (LightIntegra Technology) score as an in vitro measure for PLT quality and function was determined, which previously was shown to correlate with the corrected count increment (CCI) of PCs as a measure of posttransfusion PLT response.

Methods: PCs with a residual plasma content of 35\% were prepared from buffy coats using the additive solution SSP+ (Macopharma). PCs were pooled and split; one unit $(350 \mathrm{~mL})$ was left untreated while the other one (350 $\mathrm{mL}$ ) was irradiated on the Macotronic UV machine (MacoPharma) with a UVC dose of $0.2 \mathrm{~J} / \mathrm{cm}^{2}$. PCs $(\mathrm{n}=6)$ were stored for 7 days after preparation and samples were taken on days 2, 5 and 7. PLT- (CD 40L, RANTES, sCD62P, VEGF) and white blood cell (WBC)-associated cytokines (IL-1ß, IL-6, IL-8) were measured by enzyme-linked immunosorbent assay. The ThromboLUX score was determined by dynamic light scattering using the ThromboLUX technology (LightIntegra Technology, Inc.).

Results: All PCs (control and UVC-treated) showed low levels of WBC-associated cytokines. UVC treatment led to an only mild increase in the levels of different PLT-derived cytokines in PCs during storage. The level of RANTES in the UVC treated group was increased by $25 \%$ compared to the control group $(243.6 \pm 37.3 \mathrm{ng} / \mathrm{mL}$ vs. $305.5 \pm 31.9 \mathrm{ng} /$ $\mathrm{mL}, \mathrm{p} \leq 0.001)$ at the end of storage. Also CD40L and sCD62P were slightly higher in the UVC treated group on day 7 (CD40L $7.4 \pm 1.5 \mathrm{ng} / \mathrm{mL}$ vs. $8.9 \pm 1.7 \mathrm{ng} / \mathrm{mL}, \mathrm{p}=0.002 ; \mathrm{sCD} 62 \mathrm{P} 124 \pm 15 \mathrm{ng} / \mathrm{mL}$ vs. $137 \pm 22 \mathrm{ng} /$ $\mathrm{mL}, \mathrm{p}=0.018$ ). No significant differences in the ThromboLUX score were detected between groups. In particular, there was no statistically significant difference in microparticle concentration of the control versus the UVC-treated PCs.

Conclusion: There is only a small increase of PLT-derived cytokines in UVC-treated PCs during storage for 7 days. In addition, the ThromboLUX score of UVC-treated PLTs is well maintained. These results confirm the results of previous studies that the in vitro quality of UVC-treated PLTs is well preserved during storage.

\section{INF-P02}

The Blueflex filter of the THERAFLEX MB-Plasma system (Macopharma) efficiently removes different bacteria species from therapeutic plasma

\author{
*U. Gravemann', S. Reichenberg², C. Sumian³, A. Seltsam \\ ${ }^{1}$ DRK-Blutspendedienst NSTOB, Forschung und Entwicklung, Springe, \\ Germany \\ ${ }^{2}$ Macopharma, Langen, Germany \\ ${ }^{3}$ Macopharma, Tourcoing, Germany
}

Background: Pathogen reduction systems for fresh frozen plasma (FFP) such as the Methylene blue (MB)/light-based THERAFLEX MB-Plasma system were developed to increase the viral safety of plasma transfusions. However, FFP may also become contaminated with bacteria in rare cases. In a previous study, bacteria were efficiently eliminated from plasma by THERAFLEX MB-Plasma treatment. However, it was indeterminate what level of removal was attributed to the Blueflex filter, which as an integral to the MB-Plasma system reduces the concentration of MB and its derivatives. Methods: Plasma units $(315 \mathrm{~mL})$ were spiked with bacteria suspensions having titers of approx. $1 \times 10^{6} \mathrm{CFU} / \mathrm{mL}(S$. aureus, B. diminuta) or approx. $1 \times 10^{5} \mathrm{CFU} / \mathrm{mL}$ (B. subtilis spore preparation). The plasma was filtered using the Blueflex filter under manufacturer recommendations. Samples were taken after spiking and after Blueflex filtration. The bacteria titers were determined by plating on agar plates and the $\log _{10}$ reduction factor for the filtration was calculated.

Results: The three different bacteria species were efficiently removed from plasma by Blueflex filtration. S. aureus $(\mathrm{n}=2)$ was completely eliminated, resulting in a reduction factor of ${ }^{3} 5.9 \log$ steps. B. subtilis spores $(\mathrm{n}=2)$ were also completely removed, resulting in a $\log$ reduction of ${ }^{3} 4.7 \mathrm{log}$ steps. B. diminuta $(\mathrm{n}=4)$ which has a diameter smaller than other bacteria strains tested was not completely eliminated but removed by more than 4 log steps from FFP. 
Conclusion: The Blueflex MB removal filter has the capacity to efficiently remove bacteria from contaminated FFP. Taking into account the concentration of bacteria normally present in contaminated therapeutic plasma, the overall reduction capacity of the THERAFLEX MB-Plasma system (Plasmaflex, irradiation and Blueflex) will be sufficient to prevent transfusion-transmitted bacterial infections.

\section{INF-P03 \\ Influenza A virus H3N2 is efficiently inactivated by the THERAFLEX UV-Platelets system}

*U. Gravemann ${ }^{1}$, J. Schmidt' ${ }^{2}$ F. Tolksdorf ${ }^{3}$, C. Sumian", T.H. Müller ${ }^{5}$, G. Bauer ${ }^{6}$, S. Biczysko ${ }^{6}$, O.T. Keppler ${ }^{6}$, A. Seltsam ${ }^{1}$, H.F. Rabenau ${ }^{6}$

'DRK-Blutspendedienst NSTOB, Forschung und Entwicklung, Springe, Germany

${ }^{2}$ DRK-Blutspendedienst NSTOB, Dessau, Germany

${ }^{3}$ Macopharma, Langen, Germany

${ }^{4}$ Macopharma, Tourcoing, Germany

${ }^{5}$ DRK-Blutspendedienst NSTOB, Springe, Germany

${ }^{6}$ Klinikum der Johann-Wolfgang-Goethe-Universität, Institut für

Medizinische Virologie, Frankfurt/Main, Germany

Background: The THERAFLEX UV-Platelets system (MacoPharma) uses UVC light (wavelength: $254 \mathrm{~nm}$ ) for reduction of pathogen infectivity in platelet concentrates (PCs) without the need of any additional photoactive compound. UVC treatment selectively affects the nucleic acids of pathogens but also of leukocytes while proteins are largely preserved. It was shown previously that a variety of different viruses can be inactivated by this procedure. Aim of the study was to investigate the inactivation capacity of the THERAFLEX UV-Platelets procedure for human Influenza A virus, serotype $\mathrm{H} 3 \mathrm{~N} 2$.

Methods: First, donor sera were screened for the presence of neutralizing influenza antibodies (NIA). A double apheresis, plasma-reduced PC in additive solution $\mathrm{SSP}+(65 \% \mathrm{SSP}+/ 35 \%$ plasma $)$ was obtained from a donor who tested negative for NIA. The PC was split into two equal units that were spiked with virus suspension $(10 \% \mathrm{v} / \mathrm{v})$. The two PCs $(375 \mathrm{~mL}$ each) were UVC-irradiated on the Macotronic UV machine (MacoPharma) and samples were taken at different time points post irradiation. The infection titre of the H3N2 virus was determined by endpoint titration in microtitre plate assays on MDCK-2 cells. In most cases samples were titrated immediately, except for the "hold" sample which was stored until the end of the experiment and then titrated. For some of the samples also large volume plating was performed to lower the detection limit of the read out assay.

Results: H3N2 was highly sensitive to the THERAFLEX UV-Platelets pathogen reduction procedure. Already with a quarter $\left(0.05 \mathrm{~J} / \mathrm{cm}^{2}\right)$ of the full UVC dose $\left(0.2 \mathrm{~J} / \mathrm{cm}^{2}\right)$ a mean inactivation factor of $3.37 \log _{10}$ was achieved for UVC-treated PCs. At a UVC dose of $0.15 \mathrm{~J} / \mathrm{cm}^{2}$ and higher the virus was inactivated below the limit of detection, resulting in a H3N2 virus reduction factor of greater than $5 \log$ steps (see table).

Conclusion: Our results demonstrate that the THERAFLEX UV-Platelets procedure is an effective technology to inactivate Influenza A virus (H3N2) in potentially contaminated PCs.

Fig. 1

\begin{tabular}{|c|c|c|c|}
\hline Time-point & $\begin{array}{c}\text { UVC dose } \\
{\left[\mathbf{J} / \mathbf{c m}^{2}\right]}\end{array}$ & $\begin{array}{c}\text { mean titre } \\
{\left[\log _{\mathbf{1 0}} \mathbf{T C I D} \mathbf{5}_{0} / \mathbf{m L} \mathbf{L}\right]}\end{array}$ & $\begin{array}{c}\text { mean } \\
\log _{\mathbf{1 0}} \text { reduction factor }\end{array}$ \\
\hline Load & 0 & $5.57 \pm 0.09$ & \\
\hline Hold sample & 0 & $5.25 \pm 0.00$ & $0.32 \pm 0.42^{*}$ \\
\hline 1 & 0.05 & $1.88 \pm 0.00$ & $3.37 \pm 0.39^{* *}$ \\
\hline 2 & 0.1 & $\leq 1.5$ & $\geq 3.75 \pm 0.19^{* *}$ \\
\hline 3 & 0.15 & $\leq-0.06$ & $\geq 5.31 \pm 0.19^{* *}$ \\
\hline 4 & 0.2 & $\leq-0.06$ & $\geq 5.31 \pm 0.19^{* *}$ \\
\hline
\end{tabular}

INF-P04

\section{Prevalence of cytomegalovirus (CMV) in allogeneic cord blood units}

\author{
${ }^{*}$ A. Platz, A.-C. Aurich, A. Sauer, A.H. Schmidt, G. Ehninger \\ DKMS Nabelschnurblutbank, Dresden, Germany
}

Background: Cord blood has been established as reasonable source of hematopoietic progenitor cells for unrelated allogeneic transplantation since 1988. More than 25,000 cord blood transplantations were performed worldwide. Advantageous factors are the side-effect-free collection, lower risk of rejection compared to transplantation of bone marrow and peripheral blood progenitors and fast availability. Furthermore the risk of virus transmission is lower than in adult donors. Beside HIV, HBV, $\mathrm{HCV}$ and Treponema pallidum also cytomegalovirus (CMV) plays an important role in immunosuppressed patients.

Methods: The cohort was defined as total cord blood donations delivered at the DKMS Lifeline Cord Blood Bank between June 2011 and December 2012. Each cord blood unit meeting the inclusion criteria was processed and banked. Each cord blood unit was accompanied by maternal blood samples which were used for infectious disease markers (IDM) testing including anti-CMV - IgG and - $\operatorname{IgM}$ by enzyme immunoassay EIA. In case of seropositivity the referring cord blood sample was tested for CMV by PCR (sensitivity $125 \mathrm{IU} / \mathrm{ml}$ ). The results were compared to published data of different cohorts.

Results: 3,787 of 8,943 delivered cord blood units met all inclusion criteria for processing and banking. The corresponding maternal blood samples were tested for antibodies against CMV (IgG and $\operatorname{IgM})$. Of these 1,557 samples were seropositive, either IgG or IgM or both (41.1\%). The following PCR for CMV-detection in cord blood showed 5 samples positive $(0.32 \%)$ and 1,552 samples negative $(99.68 \%)$. The corresponding maternal results of the 5 PCR-positive samples were IgM positive only $(\mathrm{n}=1)$ and $\operatorname{IgM}+\operatorname{IgG}$ positive $(\mathrm{n}=4)$.

Conclusion: Previously published data show a prevalence of CMV-specific antibodies between 40 and $70 \%$ of adults in developed countries. The prevalence in blood donors ranges from 37 to $65 \%$ with age-dependency. The serostatus of the presented cohort is comparable to these publications. The detection of CMV in cord blood donors is with $0.32 \%$ relatively low when compared to 0.3 to $1.2 \%$ in other centers and very low in comparison to adult blood donors (2-12\%).

In accordance with other publications the DKMS Lifeline Cord Blood Bank cohort shows a low prevalence of CMV. Cord blood is superior to other stem cell sources concerning transmission of CMV infection.

\section{INF-P05}

\section{Procalcitonin in Blood Donation}

\section{${ }^{*}$ B. Wolters ${ }^{1}$, T. Keller ${ }^{2}$, H. Deiters ${ }^{1}$, A. Neumann ${ }^{1}$, K. Hartung ${ }^{1}$}

${ }^{1}$ Institut für Laboratoriums- und Transfusionsmedizin, Klinikum Bremerhaven, Bremerhaven, Germany

${ }^{2}$ ACOMED Statistik, Leipzig, Germany

Background: Bacterial contaminations of blood products are rare but in most cases severe side effects in transfusion medicine. Procalcitonin (PCT) is a new measureant making bacterial infection unlikely if the concentration is low. PCT has not been investigated in blood donors so far.

Methods: PCT (Thermo Fisher, Kryptor compact) was validated on healthy whole-blood donors $(n=1037)$. Other measureants were: blood count, CSR, CRP, GPT, GGT, syphilis-, HIV-, HBV-, HCV-serology, NAT for HIV and HCV.

A guided interview and blood culture of the buffy coat was done if CRP $\geq 10 \mathrm{mg} / 1$ or PCT $\geq 0.2 \mathrm{ng} / \mathrm{ml}$ was found.

Results: 34 (3.3\%) donors showed PCT values $\geq 0.1 \mathrm{ng} / \mathrm{ml}, 5(0.48 \%)$ donors $\geq 0.2 \mathrm{ng} / \mathrm{ml}$. The PCT upper reference limit (90.percentile) was $0.071 \mathrm{ng} / \mathrm{ml}$.

CRP $>5 \mathrm{mg} / 1$ was found in 120 whole-blood donors (11.6\%), CRP $>10$ $\mathrm{mg} / \mathrm{l}$ in 24 blood donors $(2.3 \%)$. We found common cold $(7 \times)$, rheumatic complaints $(6 \times)$, allergic coryza $(1 \times)$ and Herpes labialis $(1 \times)$ as possible 
explanations for elevated CRP. A simultaneous elevation of CRP and PCT was not found in a single case. No bacterial contaminated buffy-coat was found in these 24 cases.

All 5 donors with elevated PCT reported active athletics in the days before donation. One blood donor (male, 60 years) showed PCT of $1.032 \mathrm{ng} / \mathrm{ml}$ whereas all other laboratory results were within the reference range. It could be shown that the elevation was induced by sporting activity.

Conclusion: Bacterial contamination by bacteriamia is very rare in whole blood donation. Neither PCT nor CRP can be recommended as effective in whole-blood donation due to lacking specificity. Further studies might focus on platelet apheresis.

\section{INF-P06 \\ Comparison of three different systems for infectious disease testing}

\section{*V. Schottstedt, R. Rietz, R. Kirchhöfer, S. Droste}

DRK Blutspendedienst West, Zentrallabor, Hagen, Germany

Background: Automation of lab examinations is essential for reliable and fast result creation with regard to blood product release. We have performed a head-to-head-study to find out a suitable system to examine approx. 400 to 500 donations within 4 hours.

Methods: We have examined approx. 10,000 samples, derived from formerly negative first donations in the relevant test of record for $\mathrm{HBsAg}$, antiHBc, HCV-Ab, HIV-1/2-Ag/Ab. Samples were run on the following fully automated and innovative systems:

a) Architect i4000SR (Abbott, Delkenheim, Germany)

b) Cobas 8000 with two e602 modules (Roche, Mannheim, Germany)

c) 3 LIAISON XL (DiaSorin, Saluggia, Italy)

Cross contamination was checked by examination of alternating high positive and negative samples (3 times 12 samples each for HBsAg, $\mathrm{HCV}-\mathrm{Ab}$, resp. HIV-Ab). In addition 190 archive samples derived from deep freeze storage $\left(<-30{ }^{\circ} \mathrm{C}\right)$ with $200 \mu \mathrm{l}$ each in false bottom tubes (Sarstedt) were checked for performance of the different candidate tests. Maximum sample throughput was also evaluated. Occurring technical problems were registered. The user friendliness was evaluated.

Results: All systems performed every test once with $200 \mu 1$ archive sample volume except LIAISON XL for antiHBc resp. HIV-Ag/Ab. Turnaround time for examination of 400 samples in four tests simultaneously takes approximately: $3.5 \mathrm{~h}$ on Architect ; $4.5 \mathrm{~h}$ on Cobas 8000; and 5,5 $\mathrm{h}$ on LIAISON XL platforms respectively. In case of extended series the time to result per sample will be shortened. Technical robustness of Cobas 8000 was very well followed by LIAISON XL and Architect. Architect Software is very intuitive and easy to handle, which is nearly the same for LIAISON XL. The feature of RFID based reagent identification, which is only available on Liaison XL allows for interchanging reagent containers between different platforms while continuously tracking information about previous use is automatically documented.

Conclusion: All candidate systems revealed pros and cons during the study described. Choosing the most suitable system means to generate a cost-utility analysis in combination with defining priorities on the basis of individual preponderate demands with regard to state of the art lab automation. In our site high priority is given to the rate of false reactive results of the candidate systems causing loss of donations as well as costs for confirmatory testing. Basically all three systems can be used for donor screening in routine.

Fig. 1

\begin{tabular}{|l|c|c|c|}
\hline & $\begin{array}{c}\text { Architect } \\
\text { i4000SR }\end{array}$ & $\begin{array}{c}\text { Cobas } 8000 \\
\left.(\mathrm{e} 602)^{2}\right)\end{array}$ & Liaison XL \\
\hline HBSAg & $0,09 \%$ & $0,19 \%$ & $0,02 \%$ \\
\hline AHBC & $0,32 \%$ & $0,54 \%$ & $0,23 \%$ \\
\hline HCV-Ab & $0,18 \%$ & $0,12 \%$ & $0,36 \%$ \\
\hline HIV-1/2-Ag/Ab & $0,05 \%$ & $0,22 \%$ & $0,37 \%$ \\
\hline All & $0,64 \%$ & $1,07 \%$ & $0,98 \%$ \\
\hline
\end{tabular}

INF-P07

\section{First international proficiency panel for Hepatitis E Virus detection}

*M. Schmidt, W.J. Geilenkeuser, R. Kruse

Referenzinstitut für Bioanalytik, Vorstand, Bonn, Germany

Background: Hepatitis E virus (HEV) belongs to the Caliciviridae family and can cause hepatitis in humans like other hepatitis viruses. The main infection pathway is transmitted by infectious water (eg in Asia while monsoon) or through consumption of infectious animal meat (liver). Recently, transmissions by blood components were also reported. In each case the primary infection based on infected food, but secondary transmission by blood products were confirmed. Studies on blood donors worldwide, reported by Bailys et al. showed an incidence (RNA positive blood donors) of approximately 1:3000. Thus, the infection rate is two log periods higher than for HIV-1.

Methods: The aim of this study is the development of an international ring trial for the detection of HEV. Seven different NAT systems (4 times inhouse systems, and 3 time commercial systems; Roche s201 HEV, Novartis Panther HEV and Altona diagnostics HEV) were used by the participants. The panel included three HEV RNA positive samples with different virus concentrations (all samples were Genotype 3) and one negative sample.

Results: All participants passed the proficiency panel. False negative as well as false positive detections were not observed. The amplification efficiency between inhouse systems and commercial systems were comparable.

Conclusion: Detection of HEV in blood components is feasible by serology as well as by NAT (mini-pool NAT or individual donation NAT). Published sequences enables commercial companies as well as blood donor services with NAT experience to develop sufficient screening systems for HEV. The general introduction of HEV blood donor screening is still a point of debate in Transfusion Medicine, based on limited clinical symptoms and the option to inactivated HEV in plasma components by solvent and detergent methods.

\section{INF-P08}

\section{Hygiene Monitoring in a Hospital Transfusion Service}

\section{${ }^{\star}$ E. Strobel, P. Gleich}

Städtisches Klinikum München GmbH - Medizet, Department Mikrobiologie, München, Germany

Background: Safety of blood products from transmission of infections is achieved by providing non-infectious source material (e.g. by donor selection and laboratory testing) and by prevention of contamination during blood donation, processing, storage and application. In blood donation services a hygiene plan fixing frequency and surveillance of cleaning and disinfection measures is prescribed by federal law. But also in hospital transfusion services (HTS), according to German transfusion guidelines, a hygiene plan has to be drawn up and its realization must be documented. Methods: During the last 3 years the following equipement in our HTS (Klinikum Schwabing, Munich) was examined once a year: 1 refrigerator for red blood cell concentrates (RBCC), 2 refrigerators for plasma derivates, 1 climate box for platelet concentrates (PC), 2 freezers for fresh frozen plasma (FFP), 1 thawing device for FFP, 1 transport container for blood products and in the immunohematological laboratory 1 incubator, 2 washing centrifuges and 1 refrigerator for reagents. Bacteriological examination was done by direct contact cultures that were taken from several places of each object.

Results: The refrigerators for RBCC and plasma derivates as well as the climate box for PC showed growth of low numbers of environmental bacteria $\left(<10 \mathrm{CFU} / 25 \mathrm{~cm}^{2}\right)$. The thawing device for FFP exceeded this threshold only at the $1^{\text {st }}$ examination at 1 place $\left(11 \mathrm{CFU} / 25 \mathrm{~cm}^{2}\right)$, while the freezers for FFP showed at the $1^{\text {st }}$ and the $2^{\text {nd }}$ exmination at some places a moderately higher number of contaminants (up to $32 \mathrm{CFU} / 25 \mathrm{~cm}^{2}$ ). At the $3^{\text {rd }}$ examination the transport container showed a slightly elevated 
number of contaminants at 1 place $\left(18 \mathrm{CFU} / 25 \mathrm{~cm}^{2}\right)$. In the laboratory the washing centrifuges and the refrigerator showed only low numbers of contaminants $\left(<10 \mathrm{CFU} / 25 \mathrm{~cm}^{2}\right)$, while in the incubator at the $2^{\text {nd }}$ examination a slightly elevated $\left(13 \mathrm{CFU} / 25 \mathrm{~cm}^{2}\right)$, at the $3^{\text {rd }}$ examination a relevant $\left(70 \mathrm{CFU} / 25 \mathrm{~cm}^{2}\right)$ number of bacteria was found at 1 place.

Conclusion: Observance of the hygiene plan can be documented by written acknowledgement of every cleaning and disinfection procedure and by spot check monitoring of bacterial contamination of the equipement in the HTS. By these microbiogical examinations critical points can be detected (in our study: the refrigerators for FFP and the incubator in the laboratory) and the staff can be motivated to observe the hygiene rules.

\section{INF-P09}

\section{Prevalence of CMV-PCR positive platelet donors without antibodies in Upper Austria}

\section{${ }^{*}$ C. Fischer, M. Danzer, K. Hofer, I. Bartl, S. Böhm, J. Kaar, B. Tobisch, C. Gabriel}

Österreichisches Rotes Kreuz, Landesverband OÖ, Blutzentrale Linz, Linz, Austria

Background: The transfusion-associated risk of an infection with cytomegalovirus (CMV) by apheresis platelets (PLTs) is rather low overall. Nevertheless CMV infection in immunocompromised patients often gives rise to an invasive CMV disease. Moreover, neonatal CMV infection can lead to neonatal damage. Currently, apheresis platelets can be tested for CMV antibodies and CMV DNA screening is still not mandatory. The purpose of this study was to find out how often antibodies are not detected in CMV-PCR positive donors.

Methods: Extraction of CMV DNA from $1 \mathrm{ml}$ EDTA blood plasma was followed by real-time PCR amplification using TaqMan chemistry. The in-house PCR method including an internal control was performed using the LightCycler 2.0. Sera were tested by Enzygnost anti-CMV IgM and IgG assay using BEPIII analyzer.

Results: Between September 2009 and April 2013 a total of 9845 PLTs were tested for CMV DNA. 18 of these PLTs $(0.2 \%)$ were PCR-positive. Follow up serological testing revealed that only 7 (38.9\%) donors were seropositive for CMV antibodies.

Conclusion: In conclusion, CMV negative serological tested donations of apheresis platelets could be used to minimize the risk for transfusion transmitted CMV-infection. However, more than half of our PCR positive donors demonstrate no CMV antibodies. Therefore, based on this data we recommended CMV-PCR screening to prevent transmission of cytomegalovirus by transfusion.

\section{INF-P10}

\section{Comparison of the amount of contaminated products in the preparation of apheresis and pool platelet concentrates with a $100 \%$ apheresis platelet production}

\section{${ }^{*}$ T. Alt ${ }^{1}$, T. Thiele ${ }^{2}$, J. Bux ${ }^{1}$}

${ }^{1}$ DRK-Blutspendedienst West g GmbH, Hagen, Germany

'Universitätsmedizin Greifswald, Greifswald, Germany

Background: Transfusion of pooled platelet concentrates (PPCs) obtained from 4-6 donors may be associated with a higher risk of transfusion transmitted infections compared to single donor apheresis platelet concentrates (APCs). This may be especially relevant for an emerging HIV-like pathogen without available methods for donor screening. The purpose of this study was to investigate the arithmetical risk to release a platelet product contaminated with an HIV-like pathogen in a mixed production of $40 \% \mathrm{PPC}$ and $60 \%$ APC in Germany compared to a $100 \%$ APC supply. HIV-hemovigilance data were used as reference for the arithmetical model.

Methods: Numbers of manufactured products were retrieved from reports according to $\$ 21$ German Transfusion Act (2008-2010). It was assumed, that 2 PPCs and 10 APCs could be obtained by one infectious donor per year in average. A prevalence of 1 in 10,000 was used for an HIV-like pathogen. The prevalence of HIV-positive blood donors was retrieved from hemovigilance reports (2008-2010; §22 German Transfusion Act). The fold risk increase was determined as ratio of the sum of contaminated PCs of the mixed production and the number of contaminated PCs in case of a $100 \%$ APC supply.

Results: An HIV-like agent would result in $105 \pm 3.6$ (mean \pm SD) contaminated products using the mixed production strategy and in $49 \pm 2.3$ in case of a $100 \%$ APC production. Thus, the risk-increase between mixed production and $100 \%$ APC production is $2.13 \pm 0.02$. The known HIV frequencies result in $32 \pm 5.7$ contaminated $\mathrm{PCs}$ in the mixed production and in $27 \pm 9.1$ in a $100 \%$ APC production, indicating a risk-increase of $1.31 \pm 0.33$.

Conclusion: The amount of PCs contaminated by an HIV-like agent in a mixed production is twice as high as in a $100 \%$ APC supply based on arithmetical estimation. This differed from the risk calculated with hemovigilance data for HIV positive donors. Therefore, it is important to note that the influence of one donor on the prevalence in an APC donor population is about 60 times higher than in a PPC case. As long as there is no information about an unknown pathogen, a reliable statement about the safety of a certain production method can not be made.

\section{INF-P11}

\section{Evaluation of epidemiological reports 2. Where are the risk groups? Trends in data evaluations of the years 2006-09 and 2010-11}

\section{${ }^{\star}$ D. Jahn' ${ }^{1}$ S. Kießig², K.-P. Krause ${ }^{3}$}

${ }^{1}$ Haema AG, BSZ Marzahn, Berlin, Germany

${ }^{2}$ Haema AG, BSZ Dortmund, Dortmund, Germany

${ }^{3}$ Haema AG, HFZ, Leipzig, Germany

Background: The factors for the residual risk in the blood or plasma donating population should be evaluated comparing data sets from the years 2006-09 and 2010-11. The trends and distinctions should be evaluated.

Methods: Retrospective data sets of selected donation centers were collected for the years 2006-09 and 2010-11. The data sets of the periods 2006-09 and 2010-11 with donors who confirmed positive for an infection with HIV, HBV, HCV or Lues, were compared. Data from interviews, and donor databases were summarized, compared and descriptive statistics computed by MS-Excel and MedCalc respectively. The number of infections in the respective period were equalized to a total of $100 \%$.

Results: We found infections in 2006-9 with HIV 12.5\%, HCV 32.5\%, HBV $19.5 \%$ and Lues $35.5 \%$ (coinfections: HC/BV $1.50 \%$, HBV/Lues $0.50 \%$, HCV/Lues $0.50 \%$ ); in 2010-11: HIV 14.5\%, HCV 30.5\%, HBC $18.3 \%$, Lues $36.6 \%$ (coinfections : HI/BV $0.8 \%$, HIV/Lues $0.8 \%$ and $\mathrm{HC} /$ BV $1.5 \%$ ). The majority of donors were first time donors Most of the donors were native Germans, living in larger cities. Migration background was engaged for 18\% (2006-09) and 17\% (2010-11). A bimodal age distribution pattern was observed for HCV infection in 2006-09, but not in the data from 2010-11. For infection with HBV in 2006-9 a trimodal age distribution pattern $(18-28 ; 32-43,46-59)$ and in $2010-11$ a bimodal pattern was found $(18-15 ; 28-38)$. A constant high infection number was observed for Lues peaking at donor ages from 18-32 in both periods. Nearly a third of all infected donors had one or more tattoos or piercings. Conclusion: Known risk factors were still found underrepresented in these two data sets. To consider was the constant level of donors who not appeared for the second blood testing (2006-09 27\%, 2010-11 31.5\%). An improvement was detected in number of donors appearing in the interview to clarify the mode of infection (2006-09 45.1\%, 2010-11 59.8\%). We found an increasing number of $\mathrm{HCV}$ and $\mathrm{HBV}$ infections in the younger population which could be due to the rising number of tattoos and piercings. 
INF-P12

\section{The CCR5-delta32 polymorphism and CMV serostatus}

${ }^{*} D$. Fürst'1,2, M. Mayer ${ }^{1,2}$, C. Zollikofer ${ }^{1,2}$, H. Schrezenmeier ${ }^{1,2}$, J. Mytilineos ${ }^{1,2}$

${ }^{1}$ German Red Cross Blood Transfusion Service Baden-WürttembergHessia, Ulm, Germany

${ }^{2}$ Institute for Clinical Transfusion Medicine and Immunogenetics UIm, University of Ulm, Transplantation Immunology, Ulm, Germany

Background: The human cytomegalovirus (HCMV) US28 gene encodes a functional CC-chemokine receptor. In addition, the CMV UL33 and UL78 gene products may heteromerize with CCR5. As an interaction between CMV gene products and CCR5 seems to take place, we investigated if CMV seroprevalence is affected by the CCR5-delta32 mutation genotype (wildtype[WT] vs delta32[d32]).

Methods: CCR5 was typed in 5389 blood donors (age 40 or older) using PCR sequence specific primers. Due to differences in the age distribution between CMV seropositive and seronegative donors, substratification according to age categories was performed (age 40-49, 50-59, 60-71). Statistical analysis was performed by chisquared testing.

Results: Results are summarized in table 1. A statistically significant difference was found for donors aged 60 or older $(p=0.042)$. Donors showing the $\mathrm{d} 32$ mutation were more frequent in the CMV negative group $(22.5 \%$ vs $12.3 \%, \mathrm{p}=0.042)$. A trend was found in the age category $40-49(19.5 \%$ vs $16.5 \%, p=0.059)$. No difference was found for age category $50-59$.

Conclusion: Our data suggest that CMV infection and seroconversion rates may be influenced by the CCR5-delta32 mutation. Our analysis is limited by a low number of donors in the significant age category. Therefore, confirmation in a larger cohort should be performed.

\begin{tabular}{|c|c|c|c|c|c|c|}
\hline Age category & \multicolumn{2}{|c|}{$40-49$} & \multicolumn{2}{c|}{$50-59$} & \multicolumn{2}{c|}{$60-71$} \\
\hline CMV status & CMV- & CMV+ & CMV- & CMV+ & CMV- & CMV+ \\
\hline CCR5d32 WW & $1625(80.5 \%)$ & $792(83.5 \%)$ & $1006(81.1 \%)$ & $731(79.9 \%)$ & $79(77.5 \%)$ & $143(87.7 \%)$ \\
\hline $\begin{array}{c}\text { CCR5d32 d32MV } \\
\text { or d32/d32 }\end{array}$ & $394(19.5 \%)$ & $157(16.5 \%)$ & $235(18.9 \%)$ & $184(20.1 \%)$ & $23(22.5 \%)$ & $20(12.3 \%)$ \\
\hline p-value & \multicolumn{2}{|c|}{0.059} & \multicolumn{2}{|c|}{0.532} & \multicolumn{2}{c|}{0.042} \\
\hline $\begin{array}{l}\text { Table 1: Patient grouping according to age category, CCR5 status and CMV status, p-value from chisquare } \\
\text { testing }\end{array}$
\end{tabular}

\section{INF-P13 \\ Monocyte-Activation-Test (MAT) - a Method for Pyrogen Measurement in Pathogen Inactivated Platelets}

*U. Sicker, U. Schurig, E. Spindler-Raffel, J. Brachert, B. Löschner, I. Spreitzer

Paul-Ehrlich-Institut, Mikrobiologische Sicherheit, Langen, Germany

Background: Transfusion transmitted bacterial infections with $\mathrm{PC}$ are supposed to be prevented by Pathogen Inactivation (PI) by inhibiting microbial growth. However dying bacteria release lipopolysaccharide (LPS) respectively LTA (lipoteichoic acid) from cell wall into PC transfusion unit triggering a pyrogenic shock and probably leading to fatal reactions for the recipient. Potential other resulting irradiation products can take effect on pyrogenicity, too. This study is an approach to apply the MAT for measuring pyrogens in PCs. Methods: Pre-test: 20 PPCs are contaminated with four different strains. For each strain four PC-bags were spiked. Initial bacterial counts reached from 10 to $100 \mathrm{CFU} / \mathrm{bag}$.

At day 2, 4 and 7 samples are drawn and cryopreserved at $-20{ }^{\circ} \mathrm{C}$. In parallel colony counts by plating were performed to compare bacterial load with pyrogen concentration. Uncontaminated PC aliquots served as a negative control. PCs were stored at $22{ }^{\circ} \mathrm{C}$ under agitation.

For MAT testing cryopreserved blood and samples were added into cytokine coated 96-microwell-plates and incubated. Results were received by measuring optical density.

Study: Contamination and PI of PCs will be performed by the participating blood transfusion services. Samples will be drawn for bacterial detection (automated culture systems) and pyrogen testing. Aliquots of treated PCs will be frozen and sent to PEI to measure pyrogenicity.
Results: First results have demonstrated the feasibility of MAT. This leads, for the first time, to the opportunity to determine pyrogens in blood components, especially in PCs.

After this study is successfully completed the focus of interest will be on the MAT evaluation for Red Blood Concentrates (RBC) with subsequent detection of pyrogenicity of pathogen inactivated Erythrocytes.

In the near future MAT testing will be performed with the cryopreserved contaminated platelets. Thereafter further steps are taken to obtain an assessment about the pyrogenicity of pathogen inactivated blood.

Conclusion: PI is a tool for prolonging storage time for PCs from four to five days without bacterial screening and therefore to increase blood safety. MAT could be established as a rapid method for revealing a not successfully irradiated contaminated blood components, respectively detecting a transfusion unit with a high level of pyrogens.

In future may be MAT could serve as a tool to prevent transfusion incidents caused by pyrogens. With receiving the study results the safety for pathogen inactivated blood products can be reviewed - a benefit for blood safety.

\section{INF-P14 \\ Screening for bacterial contamination in platelet concentrates by minipool NAT}

\section{${ }^{*}$ K. Hourfar ${ }^{1}$, W. Sireis ${ }^{1}$, B. Rüster ${ }^{1}$, A. Leo ${ }^{2}$, M. Schmidt ${ }^{1}$, E. Seifried ${ }^{1}$ \\ ${ }^{1}$ DRK Blutspendedienst Baden-Württemberg - Hessen, Institut Frankfurt am Main, Spenderscreening, Frankfurt am Main, Germany ${ }^{2}$ IKTZ Heidelberg, Blutbank, Heidelberg, Germany}

Background: The residual risk of acquiring a transfusion-associated bacterial infection is significantly higher Several fatal septic reactions were reported after transfusion of platelet concentrates, predominantly involving products near the end of their shelf-life. Therefore the maximum shelf-life for platelets inGermanywas reduced from 5 days to 4 days to increase blood safety. The German competent authority for blood and blood products however allowed to re-extend the maximum shelf-life of platelet concentrates to 5 days if adequate screening methods or pathogen reduction technologies are established.

Methods: Starting in 2011 two rapid detection methods have been validated at the GRC blood donor service Frankfurt in order to enhance safety of platelet concentrates with respect to bacterial contamination. The BactiFlow (Chemunex) is a FACS based system originally developed to detect living bacteria in food. Because testing with BactiFlow is laborious, it has been widely replaced by an inhouse-developed, CE-certified NAT-system based on amplification and detection of 16s-DNA. The process of NAT-testing of platelet concentrates is performed in pools of up to 10 products per pool. Since the isolation of bacterial DNA from platelet concentrates and viral nucleic acids from minipools use the same extraction protocol on our Zelos x100 NAT-platform bacterial screening could be implemented in our routine screening without the need of additional personnel.

Results: In the validation study platelet concentrates have been spiked with $10 \mathrm{CFU}$ of several transfusion relevant bacterial strains. Both methods have proven their capability to efficiently detect bacterially contaminated platelet concentrates after at maximum 48 to 72 hours after blood donation. Until February 2013 a total of 5514 platelet concentrates have been screened by NAT. Approximately $0.5 \%$ of tests were initially reactive by NAT. Three platelet concentrates $(0.05 \%)$ were repeatedly reactive by NAT. In one of these products the reactive result could be confirmed by BacT/ALERT and blood culture. The product was found to be contaminated with Staphylococcus epidermidis and Staphylococcus warneri. Conclusion: We could show that NAT screening for bacterial contamination is feasible and improves blood safety. Due to the high grade of automation screening could be implemented without the need of additional personnel for the testing process. Additionally we could show that the rate unspecific results are much lower than expected. Due to the extended shelf life of tested platelet concentrates there is also a lower waste of blood products. Therefore our testing strategy may also be cost effective. 
INF-P15

\section{Impact of dual-target NAT-testing on blood safety - 2 years experience at GRC Frankfurt}

\author{
*K. Hourfar, G. Capalbo, W. Sireis, B. Rüster, M. Schmidt, \\ E. Seifried
}

DRK Blutspendedienst Baden-Württemberg - Hessen, Institut Frankfurt am Main, Spenderscreening, Frankfurt am Main, Germany

Background: Since introduction of minipool-NAT testing into blood donor screening at the German Red Cross it was assumed that the residual risk of acquiring a transfusion transmitted HIV-infection is mainly attributable to the transfusion of donations during the very early phase of infection. However there is additional risk due to the susceptibility of current NAT-testing systems against virus mutations. Reports have shown that several HIV-RNA positive donations were missed by NAT although the viral loads in these donations were far above the minimum sensitivity requirements in our country.

Methods: Starting in December 2010 the GRC blood donor service Frankfurt implemented dual-target testing for HIV-1. In the first step a second real-time PCR assay for HIV-1 was implemented into routine blood donor screening. Since then NAT-screening for HIV-1 RNA is performed using two individual singleplex PCR-assays targeting the 5'-LTR-region and the gag-region, respectively. Both NAT-assays exhibit comparable sensitivities of approximately 800 to $1000 \mathrm{IU} / \mathrm{ml}$ relating to an individual donation in a pool of 96 .

Results: Since December 2010 until today approximately 2.4 million donations have been screened for HIV-RNA, HCV-RNA and HBV-DNA at the GRC instituteFrankfurt. During the study period 3 donations with discrepant results between the two HIV-1-NAT assays were identified. All three donations were given by multiple time donors and were reactive in antibody-/antigen-screening using the Abbott PRISM HIV Ag/Ab Combo Assay. HIV-infection in the donors was also confirmed by immunoblot and by alternative PCR-assays. Sequence analyses of the involved virus strains are in process and will likely serve an explanation for the false negative results of NAT-testing in one of the NAT-assays.

Conclusion: Recently the Paul-Ehrlich-Institute (PEI), the German regulatory authority responsible for blood and blood products, announced to mandate the use of "dual-target" assays for HIV-blood donor screening by NAT.

Our data confirm that blood safety with regard to highly variable RNA-viruses could be improved by implementing "dual-target" NAT with amplification and detection in at least two conserved regions of the viral genome.

\section{INF-P16}

\section{A four year look back for unexpected adverse events (AEs) in the use of Methylene Blue treated plasma compared with untreated quarantine stored plasma}

\section{*W. Nussbaumer, H. Schennach, P. Mayersbach, W. Mayer} Univ.-Klinik Innsbruck, Transfusionsmeedizin, Innsbruck, Austria

Background: For fresh frozen plasma (FFP) safety can be increased by quarantine storage followed by a retesting after 4 month. Methylene-Blue (MB) treatment can increase safety of FFP by inactivating a broad spectrum of infectious agents additional to transfusion relevant accepted viruses (HIV, HCV and HBV). In contrast to a long experience of product quality (meeting quality requirements) and product tolerance by patients the French Agency for Sanitary Safety of Health products (AFSSAPS) requested the phasing out of MB treated plasma in France in December 2011 due to concerns about its safety.

Methods: In our center MB plasma (Theraflex, Macopharma, BRD) is used since 2009 , because it offers us the possibility to inactivate single donor plasma and its worldwide acceptance as a safe product for adults and infants since decades. Initiated by the AFSSAPS report we reviewed all our transfusions of MB treated plasma and compared reported AE with the rate of $\mathrm{AE}$ for our standard product, quarantine stored plasma (QP). Between January 2009 and December 2012 hospitals in our responsibility transfused 42.462 units of plasma. From $39.915(94,0 \%)$ we got a written confirmation for the transfusion. Data were integrated in our software system and analyzed separated for MB treated plasma and quarantine plasma. We compared number and quality of reported AE in total as well as for each year.

Results: 15.004 vs. 24.911 (MB vs. QP) have been reported as transfused in the observation period. $8 \mathrm{AE}$ versus $36 \mathrm{AE}$ (MB plasma vs. QP; $\mathrm{p}<$ $0,01)$ have been registered. These numbers corresponds to $1 \mathrm{AE}$ every 1.876 units transfused for MB units and 1 AE every 692 units for QP. All eight patients with reported $\mathrm{AE}$ to $\mathrm{MB}$ units received further $\mathrm{MB}$ treated plasma after the event ( 3 to 70 units) without further AE. Time span between $\mathrm{AE}$ and last MB units transfused varied between 1 and 11 days. All reported $\mathrm{AE}$ to $\mathrm{MB}$ units were classified as allergic (Pruritus 2, Urticaria 4, Flush 2, Hypotension 1, Stridor 1 and Hypertension 1), non were of serious character. One patient received $\mathrm{MB}$ treated and quarantine units at the time of $\mathrm{AE}$, a differentiation was not possible, the AE was counted for the MB group. Splitting the data by year, MB group shows lower rate of AEs but difference reached significance only in 2009.

Conclusion: Our data we do not confirm the French concerns. In contrast we found a significant lower AE rate for MB treated plasma compared with $\mathrm{QP}$, and also the character of the $\mathrm{AE}$ was in all cases of minor relevance. Splitting the data by year, significance was only seen for the year 2009. However, frequency of AEs per number of transfused units were always lower in the MB group for the whole observation period. Limitations of our data are given by the relatively low number of transfused units compared with the French data and the lack of plasma units inactivated by a different method.

\section{INF-P17 \\ S-303 treated red blood cells morphologically non-inferior to conventional red blood cells at end of shelf life}

\author{
${ }^{*}$ V. Brixner ${ }^{1}$, J. Leibacher ${ }^{1}$, S. Heldke ${ }^{2}$, B. Steffen ${ }^{2,3}$, N. Mufti ${ }^{4}$, \\ M.M. Müller ${ }^{1}$, E. Seifried ${ }^{1}$ \\ ${ }^{1}$ DRK Blutspendedienst Baden-Württemberg - Hessen gGmbH, \\ Produktion, Frankfurt, Germany \\ ${ }^{2}$ BloodCenter of Wisconsin, Applied Research Laboratory, Milwaukee \\ Wisconsin 53201-2178 USA, Germany \\ ${ }^{3}$ Klinikum Johann-Wolfgang-Goethe-Universität, Medizinische Klinik II \\ Hämatologie/Onkologie, Frankfurt am Main, Germany \\ ${ }^{4}$ Cerus Corporation, Concord, California 94520, United States
}

Background: Although the risk of transfusion-associated infections has been reduced over the past decades, blood components continue to carry a small risk of transmitting infectious diseases. This study used a pathogen inactivation system with S-303 which prevents replication of contaminating pathogens and residual leukocytes. Glutathione is included to quench non-specific reactions. Morphology has been used as a measure of the red blood cell (RBC) storage lesion as the percentage of RBCs with discoid shape decreases while the percentage of spherocytes increases.

Methods: Whole blood units (WBs) were collected from healthy volunteer donors in citrate phosphate dextrose adenine anticoagulant (CPDA). Plasmas and buffy coats were separated by centrifugation, and RBCs were suspended in saline, adenine, glucose, mannitol solution (SAG-M). Approximately half of the units were treated with S-303 and GSH for pathogen inactivation. After incubation with the S-303 and GSH at 20-25 ${ }^{\circ} \mathrm{C}$ for $18-24 \mathrm{~h}$, units were centrifuged, supernatant was discarded, and fresh SAG-M was added. The other half of the units remained untreated. All RBCs were stored under standard blood bank conditions at $4{ }^{\circ} \mathrm{C} \pm 2$. At day 35 to $38,50 \mu \mathrm{l}$ of RBCs were added to $500 \mu \mathrm{l}$ of $8 \%$ glutaraldehyde-veronal buffer to dilute and fix the RBCs.

Results: Standard smears of the samples were stained with a modified May-Grünwald-Giemsa stain and quantitatively enumerated for RBC morphology. Personnel completing the microscopic evaluation of the smears were blinded to whether the samples were treated or untreated. 
62 WBs were collected with 30 RBC units treated with S-303 while 30 remained untreated. Percentage of discocytes was lower in untreated RBCs than in S-303-treated RBCs (18.7\% \pm 13.4 vs. $29.8 \% \pm 13.5)$. Percentage of discoechinocytes was also lower in untreated RBCs than in S-303-treated RBCs (28.6\% vs. $39.5 \%)$. Results are summarized in Table 1.

A Welch Two Sample t-test was performed to evaluate the significance of the difference in discocyte percentages. The difference between the blood products was highly significant $(\mathrm{p}$

Conclusion: Enumeration was completed by oil-immersion microscopy at a magnification of $1000 \mathrm{X}$. For each smear, a total of 100 erythrocytes were counted and categorized.

S-303 treatment showed no negative effect on RBC morphology. Despite high percent variance of discocytes in the samples analyzed, the percent discocytes was significantly higher in pathogen inactivated RBC units, indicative of improved in vitro quality.

Further experiments are needed to determine the influence of S-303/glutathione and the addition of fresh SAG-M solution to the RBCs on potential functional effects and performance in vivo.

\begin{tabular}{|l|c|c|c|c|c|}
\hline Table 1 & $\begin{array}{c}\text { \% Discocytes } \\
\text { mean } \\
\text { (range) }\end{array}$ & $\begin{array}{c}\text { \% Disco-- } \\
\text { echinocytes } \\
\text { mean } \\
\text { (range) }\end{array}$ & $\begin{array}{c}\text { \% Echino- } \\
\text { cytes } \\
\text { mean } \\
\text { (range) }\end{array}$ & $\begin{array}{c}\text { \% Sphero- } \\
\text { echinocytes } \\
\text { mean } \\
\text { (range) }\end{array}$ & $\begin{array}{c}\text { \% Sphero- } \\
\text { cytes } \\
\text { mean } \\
\text { (range) }\end{array}$ \\
\hline S-303-Treated & 29.8 & 39.5 & 21.7 & 7.1 & 1.7 \\
RBCs (N=30) & $(4-65)$ & $(17-59)$ & $(1-49)$ & $(0-23)$ & $(0-5)$ \\
\hline Untreated & 18.7 & 28.6 & 39.7 & 10.6 & 2.5 \\
RBCs (N=32) & $(2-44)$ & $(14-55)$ & $(13-60)$ & $(0-31)$ & $(0-10)$ \\
\hline
\end{tabular}

\section{INF-P18 \\ Duration of Parvovirus B19 (B19V) viremia in blood donors}

\section{${ }^{*}$ D. Juhl, D. Steppat, U. Thiessen, S. Görg, H. Hennig}

Universitätsklinikum Schleswig-Holstein, Transfusionsmedizin, Lübeck/ Kiel, Germany

Background: B19V is a transfusion transmissible (TT) viral agent. As infections by blood products obtained from donors with intermediate DNA concentrations has been reported and there is evidence for a long-lasting viremia in blood donors, more date about duration and level of B19V viremia during infection are required.

Methods: Blood donors were screened for B19V DNA in minipools containing up to 96 samples by nucleic acid testing (NAT) using the cobas TaqScreen DPX test (Roche diagnostics; 95\% limit of detection [LOD]:12 IU B19V DNA $/ \mathrm{ml}$ pool plasma). In donors, who tested positive, duration and level of viremia was assessed by investigation of archive samples as well as by further follow-up testing. Whenever feasible, the period between the first positive donation and the last positive donation was determined.

Results: 77 donors (43 male, 34 female) provided 425 donations that were considered between March $8^{\text {th }} 2008$ and March $28^{\text {th }} 2013.386$ donations tested positive for B19V DNA, of those 302 had B19V DNA concentrations $<1000 \mathrm{IU} / \mathrm{ml}, 75$ had intermediate concentrations between $10^{3}$ and $10^{5} \mathrm{IU} / \mathrm{ml}$ and in 9 donations, B19V DNA concentrations exceeding $10^{5} \mathrm{IU} / \mathrm{ml}$ were detectable. $67 / 77$ donors provided more than one donation during the observation period. One donor showed probably a low-level viremia close to the LOD of the assay, resulting sometimes in positive, sometimes in negative NAT. In the remaining donors, the mean period between the first B19V DNA-positive donation and the last or the recent B19V DNA positive donation was 19.5 month, ranging from 2.3 up to 42.8 month. However, only 4 donors became negative for B19V DNA until the end of the observation period.

Conclusion: B19V viremia seems to persist considerably longer than one year in otherwise healthy blood donors. The majority of B19V DNA-positive donations hold probably no risk for transmission of B19V infection, as DNA concentration is below the limit for which TT-B19V infections are reported. However, 84 donations bore a risk for TT-B19V infections as at least intermediate $(>1000 \mathrm{IU} / \mathrm{ml}) \mathrm{B} 19 \mathrm{~V}$ DNA concentrations were detectable: a single case of a TT-B19V infection by such an intermediate viremic donations has recently been reported by Satake et al.

\section{Oral Abstract Session: Klinische Hämotherapie}

\section{KHT-V01 \\ Blood component loss in blood establishments and hospitals}

\section{${ }^{*}$ O. Henseler, M. Heiden, B. Haschberger, R. Seitz}

Paul-Ehrlich-Institut, Hämatologie / Transfusionsmedizin, Langen, Germany

Background: Blood components for transfusion are lifesaving medicines made from donations of non-remunerated voluntary donors. Handling with reasonable care is self-evident. Nevertheless, due to its short shelf life blood components may expire.

Methods: Blood establishments (BE) and hospitals annually report data on manufacture, use and loss of blood components to the Paul-Ehrlich-Institut via web-application. The number of hospital beds, taken from the hospital's quality reports 2010 published in www.deutsches-krankenhaus-verzeichnis.de, was used to classify hospital size.

Results: 4.5 million red blood cell concentrates (RBC) were provided in 2012. The loss rate in BE decreased to $2.5 \%$. 4.1 million RBC were transfused, $7.8 \%$ thereof by practitioners. The overall portion of loss in medical facilities was $3.5 \%$. In some cases it was not evident if none of the RBC expired or if expiry was not reported. Evaluation of data from facilities which reported use as well as loss showed a loss rate of 3.8\%. The median per hospital loss rate was 3.0\% (25\% percentile $1.5 \%, 75 \%$ percentile $6.4 \%$ ). The situation is similar for platelet concentrates (PC). 0.54 million PC were released in $2012,62 \%$ thereof by apheresis donations. In BE $12.4 \%$ of Pool-PC and $5 \%$ of apheresis PC were discarded. 0.49 million PC were reported as transfused. The overall loss rate in the medical facilities decreased slightly to $4.7 \%$. Data from facilities which reported both, use and loss of PC, showed a loss rate of $5.4 \%$. In median $3.9 \%$ (25\% percentile $1.7 \%, 75 \%$ percentile $9.0 \%$ ) expired per hospital. Conclusion: Data show a weak negative correlation between hospital beds and loss rate (RBC: $r_{s}=-0.24,95 \%$ confidence interval -0.30 to -0.19 , PC: $r_{s}=-0.21,95 \%$ confidence interval -0.29 to -0.12 ). However, per hospital loss rates are widely spread even in smaller hospitals with less than 300 beds (fig. 1 and 2). The overall loss rates decreased at both $\mathrm{BE}$ and users. Logistics in major hospitals may allow a more efficient reduction of the loss of blood components. The wide range of data variability does not allow further conclusions.

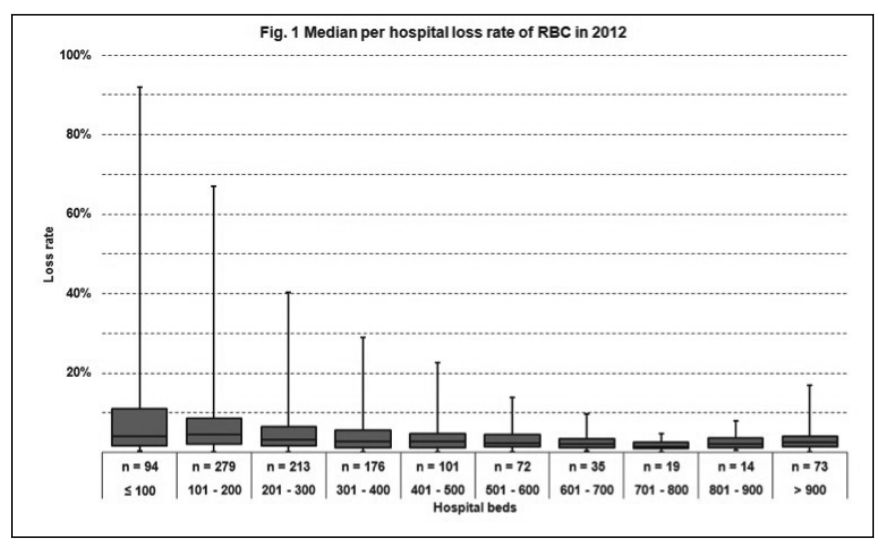




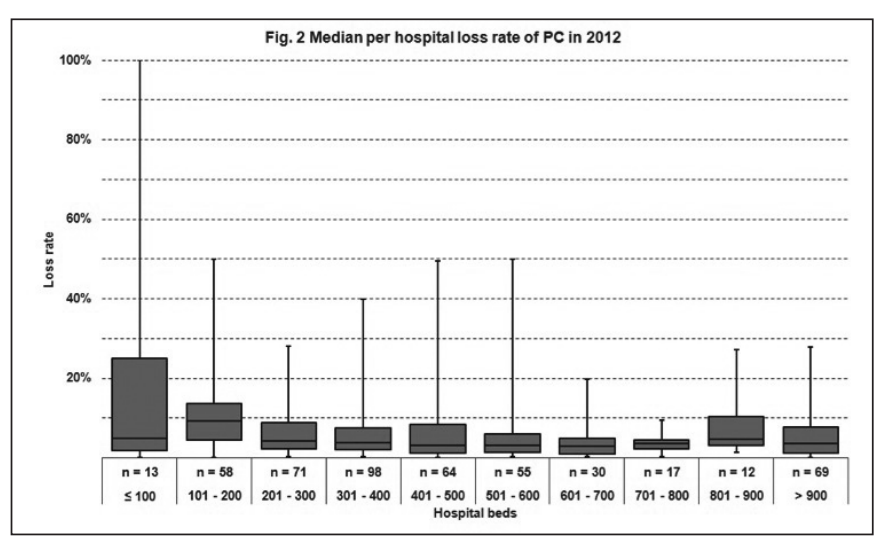

KHT-V02

Utilization of Blood Components in Abdominal Surgery and Gastroenterology: Impact of a Patient Blood Management Initiative

${ }^{*}$ G. Geißler ${ }^{1}$, D. Franz ${ }^{2}, H$. Buddendick ${ }^{2}, H$. Bunzemeier ${ }^{2}$ N. Roeder ${ }^{3}$, M. Colombo-Benkmann ${ }^{4}$, H. Ullerich ${ }^{5}$, P. Schlenke', W. Sibrowski ${ }^{1}$

${ }^{1}$ Univ. Klinikum Münster, Transfusionsmedizin u.

Transplantationsimmunologie, Münster, Germany

${ }^{2}$ Univ. Klinikum Münster, Med. Management, Münster, Germany

${ }^{3}$ Univ. Klinikum Münster, Klinikvorstand, Münster, Germany

${ }^{4}$ Univ. Klinikum Münster, Allgemeine Chirurgie, Münster, Germany

5Univ. Klinikum Münster, Med. Klinik B (Gastroenterologie), Münster, Germany

Background: As in other countries of the western hemisphere, an ageing of the German population can be observed. One consequence is that patients get older and may need more blood components than younger persons. As another consequence the number of younger people who notably are recruited as healthy blood donors declines which could result in future shortage of the blood components. Beyond that, intensified therapeutic procedures lead to increased consumption of blood components, especially in hospitals of maximum care.

Methods: At the UKM all blood components comprising red blood cells (RBC), fresh frozen plasma (FFP) or platelets (PLT) used for in-patients are documented and attributed to clinical performance groups and detailed clinical subgroups by continuous data processing. As a part of a hospital-internal patient blood management (PBM) program, this system allows to monitor the utilization of all blood components in detail, e.g. in different abdominal diseases in the department of visceral surgery and of gastroenterology (here from 2009 to 2012).

Results: The PBM at the UKM was aimed to augment the recruitment of long-term blood donors and to avoid inappropriate blood transfusions. A periodic reporting system on the demand of blood components was established to gain a higher level of transparency for the responsible clinicians. Based on stable in-patients cases numbers, exemplarily the number of surgical patients with transfusions (2009 23.7\% / 7.7\% cases with RBC / FFP transfusions resp., 2012 18.0\% / 6.8\% cases with RBC / FFP transfusions resp.) and the number of used products decreased to RBC 56.9\%, FFP $50.0 \%$, PLT $42.3 \%$ in $2012(2009=100 \%)$. However, in non-surgical treatments, the only tendency in reducing FFP and PLT consumption could be observed. Concomitantly, transfusional training courses for doctors and nurses were performed to teach the hospital-internal standards and to achieve a higher level of awareness to make the decision for a blood transfusion and to perform a blood transfusion accurately.

Conclusion: In the scenario of possible scarcity of blood components, PBM is able to ameliorate the blood supply situation, exemplarily shown here in the field of abdominal diseases. Training courses and periodic reports on transfused patients increase the awareness of "all participating practioners" in dealing carefully with blood components. An interdisciplinary dialogue between experts of transfusion medicine and clinical de- partments may help to further clear the indications of blood transfusions and reduce its non-appropriate use.

\section{KHT-V03}

No benefit from intensified coagulation factor application in cardiovascular surgery and solid organ transplantation

\section{S. Tellkamp ${ }^{1}$, D. Scheinichen ${ }^{1}$, A. Tiede ${ }^{1}$, R. Blasczyk ${ }^{2}$, ${ }^{*}$ H.-G. Heuft ${ }^{2}$}

${ }^{1}$ Medizinische Hochschule Hannover, Hannover, Germany

${ }^{2}$ Medizinische Hochschule Hannover, Institut für Transfusionsmedizin,

Hannover, Germany

Background: Bleeding complications may contribute to major morbidity and mortality after surgery. Coagulation management including fibrinogen (F. I), fibrogammin (F. XIII), prothrombin complex (PPSB) or antithrombin (AT) might avoid bleedings, reduce blood transfusions and could lead to a beneficial clinical outcome. We analyzed the need for blood components and clinical outcome in patients with major cardiovascular surgery or solid organ transplantation (TX) who were treated with increasing doses of coagulation factor concentrates.

Methods: In a retrospective comparative study, 795 patients of Hannover Medical School $(2007, \mathrm{n}=312 ; 2009, \mathrm{n}=483)$ were investigated for the usage of blood components (red cells [RC], therapeutic plasma [TP], and platelets [PC]) and coagulation factors such as F.I, F.XIII, PPSB and AT with regard to clinical factors (e. g. intensive care unit hospital stay [LO$\left.\mathrm{S}_{\mathrm{ICU}}\right]$, survival). Of the 795 patients, 611 underwent 5 categories (cat) of surgical interventions: cat I, combined heart/aortic surgery, $\mathrm{n}=80,2007$; $\mathrm{n}=98,2009$; in total 178 patients; cat II, heart arteries bypassing and/or heart valve surgery, partly combined $n=52,2007 ; n=164,2009$; in total 216 patients; cat III, heart or lung TX, partly combined, $\mathrm{n}=33,2007$; $\mathrm{n}=45,2009$; in total 78 patients; cat IV, artificial heart TX, $\mathrm{n}=18,2007$; $\mathrm{n}=31,2009$, in total 49 patients; cat V, liver $\mathrm{TX}, \mathrm{n}=52,2007 ; \mathrm{n}=38$, 2009 , in total 90 patients.

Results: Selected results are shown in the table. From 2007 to 2009 the application of F. I, F. XIII, PPSB and AT substantially increased by medians of $25 \%$ for AT to $68 \%$ for PPSB. In contrast, RC transfusions remained stable in all, TP and PC transfusions in 4 out of 5 surgical intervention categories. Regarding clinical outcome, $\operatorname{LOS}_{\mathrm{ICU}}$ rose in 4 of 5 categories while survival did not significantly change in 2009 as compared to 2007.

Conclusion: Overall, the sharp increase in the application of coagulation factors did neither reduce blood transfusion needs nor improve clinical outcome.

\begin{tabular}{|l|c|c|c|c|c|c|}
\hline & \multicolumn{3}{|c|}{2007} & \multicolumn{3}{c|}{2009} \\
\hline & $\begin{array}{c}\text { F. I } \\
(\mathrm{g})\end{array}$ & $\begin{array}{c}\text { RC } \\
(\mathrm{n})\end{array}$ & $\begin{array}{c}\text { Survival } \\
(\%)\end{array}$ & $\begin{array}{c}\text { F. I } \\
(\mathrm{g} ; \mathrm{p} \text {-value })\end{array}$ & $\begin{array}{c}\text { RC } \\
\text { (n; p-value) }\end{array}$ & $\begin{array}{c}\text { Surviva } \\
1(\%)\end{array}$ \\
\hline Cat I & $6.7 \pm 0.6$ & $12 \pm 1$ & 90 & $9.5 \pm 0.8 ; \leq 0.007$ & $12 \pm 1 ;=0.80$ & 78 \\
\hline Cat II & $3.8 \pm 0.5$ & $9 \pm 1$ & 85 & $5.4 \pm 0.4 ; \leq 0.02$ & $11 \pm 1 ;=0.22$ & 81 \\
\hline Cat III & $8.6 \pm 1.4$ & $28 \pm 4$ & 70 & $8.2 \pm 1.0 ;=0.79$ & $21 \pm 3 ;=0.15$ & 78 \\
\hline Cat IV & $6.1 \pm 1.1$ & $17 \pm 3$ & 89 & $8.9 \pm 1.4 ;=0.18$ & $14 \pm 2 ;=0.37$ & 90 \\
\hline Cat V & $4.8 \pm 0.6$ & $21 \pm 3$ & 81 & $\begin{array}{c}11.5 \pm 2.0 ; \\
\leq 0.000\end{array}$ & $21 \pm 2 ;=0.61$ & 77 \\
\hline
\end{tabular}




\section{KHT-V04}

\section{The effect of plasma exchange and immunoadsorption} with tryptophan column on anti-Xa activity

\author{
*J. Kößler, A. Kobsar, S. Kuhn, A. Kößler, P. Yilmaz, E. Weinig, \\ E. Putz, M. Böck, E. Klinker
}

University of Würzburg, Institute of Transfusion Medicine and Haemotherapy, Würzburg, Germany

Background: Plasma exchanges (PE) and immunoadsorptions (IA) are used to remove autoantibodies from the plasma in order to treat neurological autoimmune disorders. The impact of these procedures on anti-Xa activity in patients under low molecular heparin (LMWH) therapy has not been thoroughly evaluated yet.

Methods: In five patients with neurological diseases and LMWH therapy, anti-Xa activity and global coagulation parameters were measured before and after the procedures (11 PEs and additionally five IAs in two of the patients) on a BCS XP analyzer (Siemens Healthcare Diagnostics, Marburg, Germany). Therapies were performed on a cell separator (Cobe Spectra or Spectra Optia, Terumo BCT, Lakewood, CO, USA) with ACD-A as anticoagulant (ratio 1:12). In PE, plasma was substituted by electrolyte/HES solutions and 5\% albumin (with a relation of about $1: 1$ ). During IA, 2300 to $2500 \mathrm{~mL}$ plasma was directed on a tryptophan column (Immusorba TR-350, Asahikasei Kuraray Medical Immusorba, Japan) Student's t-test for paired samples was used for comparison.

Results: After plasma exchanges, aXa activity was significantly reduced from $0.57 \pm 0.10 \mathrm{IU} / \mathrm{mL}$ (mean \pm SEM) to $0.13 \pm 0.05 \mathrm{IU} / \mathrm{mL}$ (p < $0.0001)$, whereas aXa levels were not affected by IA $(0.88 \pm 0.17 \mathrm{IU} / \mathrm{mL}$ before and $0.83 \pm 0.14 \mathrm{IU} / \mathrm{mL}$ after IA, $\mathrm{p}=0.4686)$. Antithrombin activity decreased from $102 \pm 4 \%$ to $42 \pm 2 \%$ in PE $(\mathrm{p}<0.0001)$ and only slightly from $95 \pm 8 \%$ to $85 \pm 1 \%$ in IA $(\mathrm{p}=0.3820)$. Prothrombin time was reduced from $87 \pm 3 \%$ to $42 \pm 2 \%$ in PE $(\mathrm{p}<0.0001)$ and from $74 \pm 6 \%$ to $47 \pm 6 \%$ in IA ( $p=0.0018)$. aPTT values increased from $41.4 \pm 2.8 \mathrm{~s}$ to $79.1 \pm 6.4 \mathrm{~s}$ in PE $(\mathrm{p}<0.0001)$ and from $50.8 \pm 2.1 \mathrm{~s}$ to $96.8 \pm 9.1 \mathrm{~s}$ in IA $(\mathrm{p}=0.0041)$.

Conclusion: In contrast to $\mathrm{PE}, \mathrm{aXa}$ activity and antithrombin activity are almost completely preserved after IA with tryptophan column. In conclusion, patients with LMWH therapy are exposed to stronger anticoagulation after IA compared to $\mathrm{PE}$. In context with the reduction of coagulation factors, especially fibrinogen, the different impact of the two procedures on aXa activity must be obeyed in dosing and monitoring of LMWH therapy to minimize the bleeding risk.

\section{KHT-V05}

\section{Successful use of eculizumab for treatment of an acute hemolytic reaction after $\mathrm{ABO}$ major incompatible red cell transfusion}

\section{${ }^{*}$ C. Weinstock ${ }^{1,2}$, K. Weisel ${ }^{3}$, C. Dorn ${ }^{3}$, R. Möhle ${ }^{3}$, B. Höchsmann ${ }^{1,2}$, H. Schrezenmeier ${ }^{1,2}$, L. Kanz $^{3}$}

${ }^{1}$ University of Ulm, Institute of Transfusion Medicine, Ulm, Germany ${ }^{2}$ German Red Cross Blood Service Baden-Württemberg - Hessen, Institute of Clinical Transfusion Medicine and Immunogenetics, UIm, Germany

${ }^{3}$ University Hospital of Tuebingen, Department of Medicine, Tuebingen, Germany

Background: Eculizumab (Ecu) is a monoclonal antibody directed against complement factor 5 (C5). It prevents its cleavage and the formation of the membrane attack complex (MAC). It is registered for treatment of PNH and atypical HUS. It was hypothesized that Ecu might also be beneficial after ABO-major incompatible transfusion of red blood cell concentrates (RBC). Here we report a case of instant use of Ecu after a transfusion error.

Methods: When a PNH pat. (not treated with Ecu) with blood group (BG) B was erroneously transfused with about $180 \mathrm{ml}$ of RBC of BG A(2) and a haemolytic reaction started, Ecu was administered immediately after the transfusion error was noted. The pat. was monitored serologically for 4 months. ABO grouping and the direct antiglobulin test (DAT) were performed with a gel column agglutination method (Grifols; Langen, Germany). FITC-labelled anti-A was added to pat. blood samples and the amount of BG A RBC was tested by FACS. In a second, independent approach BG A RBC were co-stained with the GPI-anchored marker CD59 to distinguish between the transfused RBC and the pat.'s PNH red cells. Results: The pat. received $250 \mathrm{mg}$ prednisolone and $900 \mathrm{mg} \mathrm{Ecu} \mathrm{im-}$ mediately (Ecu dose repeated after 7 days). Signs of hemolysis (gross hemoglobinuria) improved very rapidly after Ecu infusion, the pat. remained clinically stable, no renal failure, no DIC, only transient bilirubin increase, and even decline of LDH were observed. The anti-A titer was 2 and did not increase over time. After transfusion, up to $19 \% \mathrm{RBC}$ with BG A were detectable. The incompatible cells survived surprisingly long in the pat.'s blood: after 4 days $16 \%$, after 3 weeks $4 \%$, and after 8 weeks still 1,2\% RBC had BG A. FACS analysis confirmed a large PNH clone. Double staining with anti-A and anti-CD59 identified the transfused RBC (BG A positive, CD59 positive, i.e. non-PNH cells) and this independent approach confirmed the long persistence of transfused RBC (BG A / CD59 positive). The relative proportion of $\mathrm{PNH}$ cells remained stable in the week after transfusion. Prior to transfusion the DAT was negative and became weekly positive for $\mathrm{C} 3$ after transfusion, but remained negative for IgG. After 2 weeks anti-C3 reacted more strongly and this lasted until the end of the observation period.

Conclusion: The clinical course of this acute haemolytic reaction was very mild and the pat. recovered without sequelae. This might in part be due to the low anti-A titer of 2 and the transfusion of BG A(2) RBC. In addition, intravascular hemolysis by $\mathrm{IgM}$ most likely was stopped by Ecu. The long persistence of BG A RBC supports the effect of Ecu in this case. Foremost all efforts must be undertaken to avoid transfusion errors. However, if an ABO major incompatible RBC transfusion happens, the immediate off-label administration of Ecu might help to limit the consequences of this potential life-threatening situation. A prospective trial of Ecu after ABO-incompatible transfusion errors might be considered.

\section{KHT-V06}

\section{Case Report: Gestational Alloimmune Liver Disease (GALD)}

Treatment of a newborn by pre- and postnatal IVIGadministration and exchange transfusion

\section{${ }^{*}$ S. Unkrig ${ }^{1}$, A. Hansmann², A. Müller², J. Oldenburg1, J. Hoch ${ }^{1}$}

${ }^{1}$ Inst. f. Exp. Hämatologie u. Transfusionsmedizin, Bonn, Germany ${ }^{2}$ Zentrum für Kinderheilkunde, Neonatologie, Bonn, Germany

Background: Liver failure in the newborn may be caused by a fetomaternal alloimmune condition until recently referred to as neonatal haemochromatosis. This rare disease is characterized by severe liver function insufficiency accompanied by siderosis. Maternal alloantibodies directed to fetal liver cells are supposed to cause complement mediated cell destruction. Survival rates without liver transplantation have been reported to be less than $20 \%$ and the outcome of early liver transplantation is doubtful. Exchange transfusion and IVIG have hence been established as therapeutic options.

Methods: A 31-year-old woman, gravida 4 para 3, was admitted to our clinic after 36 weeks of gestation and underwent Caesarean section due to cardiotocographic findings. One year before, the family's third child had died from liver failure at the age of six weeks and neonatal haemochromatosis had been histologically confirmed. During the present pregnancy, IVIG had been weekly administered to the mother in our antenatal centre from week 22 of gestation until delivery. Nevertheless, immediately post-delivery, the male neonate showed severe coagulation factor deficiency, hypoalbuminemia and severe hypoglycemia while ferritin was high. Laboratory and sonographic as well as clinical findings were compatible with GALD.

Results: To prevent bleeding, fresh frozen plasma and vitamin $\mathrm{K}$ were administered shortly after delivery. Exchange transfusion was performed on day 2 and 4 to remove maternal IgG. Coagulation markers and ferri- 
tin levels improved dramatically after these measures and slowly came close to normal. At the age of three weeks the baby was discharged from clinical care.

Conclusion: Anamnesis and clinical course of the case described here confirm the extraordinary importance of early therapeutic intervention in GALD even when antenatally IVIG was administered. The diagnosis GALD should be considered in every newborn with severe liver disease. high ferritin and congenital bleeding disorders. Where the condition is known antenatally, IVIG-administration during pregnancy and postnatal exchange transfusion seem adequate measures to reduce the risk of lethal liver failure. Eculizumab (Anti-C5) should be discussed as an alternative treatment option.

\section{Poster: Klinische Hämotherapie}

\section{KHT-P01}

\section{Algorithm-guided preoperative anemia management using Thomas-plot as diagnostic tool in patients undergoing elective major orthopedic surgery}

${ }^{*}$ D. Enko ${ }^{1}$, F. Wallner', A. von Goedecke ${ }^{2}$, C. Hirschmugl, V. Auersperg ${ }^{3}$, G. Halwachs-Baumann ${ }^{1}$

${ }^{1}$ Landeskrankenhaus Steyr, Institut für medizinische und chemische Labordiagnostik, Steyr, Austria

${ }^{2}$ Landeskrankenhaus Steyr, Institut für Anästhesiologie und

Intensivmedizin, Steyr, Austria

${ }^{3}$ Landeskrankenhaus Steyr, Abteilung für Orthopädie und orthopädische Chirurgie, Steyr, Austria

Background: Patient blood management has developed into a multidisciplinary clinical concept in the last years. Preoperative anaemia management is one essential part of this strategy. The purpose of this study was to evaluate a laboratory-guided (Thomas-plot) therapeutic algorithm of preoperative anaemia. The effects on various laboratory parameters, need of blood and therapeutic costs were investigated.

Methods: 335 patients with scheduled hip or knee arthroplasty were included in this retrospective before and after study. Group I $(n=101)$ underwent conventional preoperative clinical investigation before algorithm-implementation. Group II $(n=234)$ underwent the diagnostic algorithm with the Thomas-plot using reticulocyte haemoglobin content (CHr) and soluble transferrin receptor (sTfR)/log ferritin ratio as biochemical markers. Iron deficiency (ID) was substituted with $1000 \mathrm{mg}$ iron intravenous (i.v.) $+10,000$ international units (I.U.) erythropoiesis stimulating agent (ESA) subcutaneous (s.c.) or i.v.. Anaemia of chronic disease (ACD) (without functional ID) was substituted with $200 \mathrm{mg}$ iron i.v. +40000 I.U. ESA s.c. or i.v.. Hb-level of $13 \mathrm{~g} / \mathrm{dl}$ was the therapeutic cut-off for men as well as women. Thomas-plot was compared with published Network for Advancement of Transfusion Alternatives (NATA) guidelines.

Results: 56 patients (Group I: $\mathrm{n}=24$, Group II: $\mathrm{n}=32$ ) showed preoperative anaemia. $71.4 \%$ presented ACD, $19.6 \%$ latent ID and $9 \%$ manifest ID respectively. In Group II therapeutic intervention increased preoperative Hb-level (mean $\uparrow 0.58 \mathrm{~g} / \mathrm{dl}$ ), mean corpuscular volume (MCV) (mean $\uparrow$ $0.63 \mathrm{fl})$, mean corpuscular haemoglobin $(\mathrm{MCH})$ (mean $\uparrow 0.39 \mathrm{pg}), \mathrm{CHr}$ (mean $\uparrow 1.04 \mathrm{pg}$ ) and ferritin (mean $\uparrow 115.69 \mathrm{ng} / \mathrm{ml}$ ). Need of blood in the substituted group was reduced by $44 \%$. In both groups therapeutic costs were equal. Using NATA guidelines, $50 \%$ of preoperative anaemia in Group II could not be categorized, compared to Thomas-plot guided diagnosis.

Conclusion: Thomas-plot as diagnostic tool is well adapt to differentiate ID from ACD or combined ID/ACD in patients with preoperative anaemia. Laboratory-guided preoperative anaemia management optimizes perioperative $\mathrm{Hb}$-level and reduces peri- and postoperative need of blood.

\section{KHT-P02}

\section{Management of severe Sickle cell disease: Is supportive treatment with hyperbaric oxygen therapy an option?}

\section{*K. Strathmann', U. Germing ${ }^{2}$, A. Raffel ${ }^{3}$, G. Schieren ${ }^{4}$,} H. Strelow ${ }^{5}$, R. Deitenbeck ${ }^{6}$, R. Scharf

${ }^{1}$ Department of Hemostasis, Hemotherapy and Transfusion Medicine Heinrich Heine University Medical Center, Duesseldorf, Germany 2Department of Hematology, Oncology and Clinical Immunology, , Heinrich-Heine-University Medical Center, Duesseldorf, Germany ${ }^{3}$ Department of General-, Visceral- and Pediatric Surgery, Heinrich-HeineUniversity Medical Center, Duesseldorf, Germany ${ }^{4}$ Department of Nephrology, Heinrich-Heine-University Medical Center , Duesseldorf, Germany

${ }^{5}$ Department of Trauma and Hand Surgery, Hyperbaric Oxygen Therapy, Heinrich-Heine-University Medical Center, Duesseldorf, Germany ${ }^{6}$ German Red Cross Blood Service West, Center of Transfusion Medicine, Hagen, Germany

Background: Sickle cell disease (SCD) is the most prevalent genetic disorder in the Africans and Afroamericans. The main strategy of the treatment of sickle cell crisis is RBC transfusion. Haemotherapy in patients with an irregular alloantibody anti- $\mathrm{U}$ is challenging, due to the high frequency of the U-antigen in Caucasians (99,9\%), Africans and Afroamericans $(99 \%)$. In the patient reported here, we have evaluated supportive means such as erythropoietin and hyperbaric oxygen therapy to maintain sufficient oxygenation.

Methods: In 3/2011, the patient had a sickle cell crisis and required transfusion of 6 RBC overall. At that time the irregular antibody anti- $U$ was detected. The patient was in a stable clinical condition for the next months. In 03/2012 an emergency operation because of a mechanical ileus was required with segmental resection of the left colon transversum. Ten days after surgery an additional segmental resection of the colon transversum was performed, followed by a reoperation because an anastomosis insuffiency. All surgical interventions were performed without relevant blood loss. From 3 through 5/2012, 7 RBC were required because of slight but ongoing hemolysis. In May 2012 hemodialysis was started because of chronic renal failure. From 5 through 7/2012, 8 RBC were transfused. So an increasing $\mathrm{RBC}$ requirement was obvious.

Results: The U-negative RBC were supplied by the French National Institute of Blood Transfusion (INTS), Paris (12 RBC), the German Red Cross Blood Service West, Hagen (7 RBC), the NHS Blood Bank in Liverpool (1 RBC) and the Sanquin Blood Bank Amsterdam (1 RBC). In Germany only one compatible U-neg. active blood donor was available. The hemodialysis was performed three times a week and the patient received erythropoietin (Aranesp ${ }^{\circledR} 300 \mu \mathrm{g}$ ) one dose per week. In 7/2012 $\mathrm{RBC}$ were been transfused. Because of ongoing severe anemia a trial with the hyperbaric oxygen therapy (HBO) was started in 7/2012 five times a week. After 7 month the frequency of the $\mathrm{HBO}$ was reduced to 2-3 times a week. Since the beginning of $\mathrm{HBO}$, the hemoglobine concentration remained at values between 5 and $7 \mathrm{~g} / \mathrm{dl}$. No further RBC transfusions were required.

Conclusion: A patient with sickle cell anemia, alloantibody anti-U and additional causes of an anemia in a chronic renal failure requires longterm support for a sufficient oxygenation. The availability of compatible U-negative RBC is very limited. In this case, the hyperbaric oxygen therapy was an effective inexpensive promising option. 
KHT-P03

\section{Ambulant granulocyte transfusions - a case report}

${ }^{*}$ C. Kreissig 1 , I. Rehfeld ${ }^{1}$, A. Reil', R. Noppeney 3 , J. Bux ${ }^{4}$

${ }^{1}$ DRK-BSD West gGmbH, Zentralbereich Stammzelle, Ratingen Breitscheid, Germany

2DRK-BSD West gGmbH, Leukozytenimmunologie, Hagen, Germany ${ }^{3}$ Universitätsklinikum Essen, Insitut für Hämatolgoie, Essen, Germany ${ }^{4}$ DRK-BSD West gGmbH, Hagen, Germany

Background: Granulocyte transfusions have been successfully applied to neutropenic patients with severe infections, which were progressive despite the use of appropriate antibacterial and/or fungal agents.

We treat for the first time a patient suffering from chronic granulomatous disease with ambulant granulocyte transfusions as supportive adjuvant therapy before allogeneic stem cell transplantation. The ambulant therapy was chosen to reduce long hospitalization of the patient and to minimize the risk of new infections.

Methods: A 21-year-old male patient suffered from chronic granulomatous disease (CGD) and sepsis with pneumonia, caused by Pneumocystis jirovecii and multiple liver abscesses caused by Escherichia coli. After admission in hospital with high fever the patient was treated with Meropenem, Metronidazole, Ambisome and Cotrimoxazole. Under this therapeutic regime abscesses in lung and liver began to disappear. Laboratory signs of infection normalized and fever disappeared. Seven days later fever appeared again. CT-lung inspections showed lung affection. Despite of broad antibiotic therapy general condition and respiratory condition worsened. Therefore, the patient received for 14 days, every second day, transfusions of granulocytes. Seven HLA unmatched granulocyte concentrates from 2 healthy donors were given to the patient.

Results: Compatibility was tested by cross-matching the donors's leukocytes with the patient's plasma in granulocyte and lymphocyte immunofluorescence test, as well as granulocyte agglutination test.Under this therapy the patient became better. Laboratory signs of infections and fever disappeared. Ten days later, the patient's clinical condition worsened again and he was transferred to the intensive care unit. This time the infection could be controlled by administration of Meropenem. To avoid further severe infections, it was decided to establish an ambulant therapy with Meropenem and granulocyte transfusions until allogeneic stem cell transplantation.

Conclusion: Granulocyte transfusions are useful in the therapy of bacterial/fungal infections of CGD patients. Since patients with CGD frequently develop leukocyte antibodies, thorough leukocyte cross-matching is required to prevent acute transfusion-related lung injury.This approach makes ambulant transfusion possible.

Patients benefit from lower rates of nosocomial infections and mental stability in their domestic surround.

\section{KHT-P04}

\section{The Transfsuion of Blood Product in Internal Medicine, Surgery and Intensive Care Medicine in Hospitals of a Hospatal Association in Saxony}

\author{
${ }^{*}$ R. Findeisen ${ }^{1}$, E. Guhr ${ }^{2}$, B. Schmoz ${ }^{3}$ \\ ${ }^{1}$ Oberlausitz-Kliniken, Institut für Klinische Chemie und \\ Transfusionsmedizin, Bautzen, Germany \\ ${ }^{2}$ Labor Dresden-Elsterwerda, Dresden, Germany \\ ${ }^{3}$ ELBLAB GmbH, Labor, Meissen, Germany
}

Background: This research project investigated the transfusion of blood products in the hospitals of a hospital association from 2005 to 2010. The hospital association is a clinical in a hospital network composed by four units, with a total of 1.200 beds. This complex comprehends wards for Internal Medicine, Surgery, Orthopaedics and Traumatology, Gynaecology and Obstetrics, Paediatrics, Psychiatry, Ophthalmology, Oto-Rhino-Laryngology and Urology.

Methods: The clinics of the four hospitals of the hospital association were combined to the following three medical disciplines: a) Predominantly internal working disciplines [Internal Medicine; Paediatrics, Psychiatry, Ophthalmology, Oto-Rhino-Laryngology]; b) Predominantly surgical working disciplines [Surgery, Orthopaedics and Traumatology, Gynaecology and Obstetrics, Urology] and c) Intensive care.

The transfusion of the following blood products was statistically evaluated from 2005 to 2010: heterologous and autologous red blood cells (RBCs); heterologous and autologous fresh frozen plasma (FFP); platelets; prothrombine complex (PPSB), Antithrombin III (ATIII) and Fibrinogen.

Results: In all three disciplines an increase in the transfusion of heterologous RBCs was observed from 2005 to 2010. For instance was the increase from 2005 to 2010 in Hospital A 26 percent; in Hospital B 295 percent, in Hospital C 3 percent and in hospital D 32 percent. The increase of platelets was from 2005 to 2010 in Hospital A 146 percent, in Hospital B 40 percent, in Hospital C 132 percent and in Hospital D over 500 percent. The transfusion of PPSB was different in the 4 Hospitals. For instance from 2005 to 2010 in Hospital A was a decrease of 6 percent, in Hospital B a decrease of 70 percent and in Hospital C a decrease of 34 percent. Only Hospital D shows an increase of 96 percent in transfusion of platelets. While transfusions of Fibrinogen increased, the number of transfusions of ATIII significantly decreased. For instance was the decrease from 2005 to 2010 in Hospital A 31 percent; in Hospital C 63 percent and in hospital D 92 percent.

Conclusion: On the one hand, the results of this investigation reflect the new guidelines in transfusion medicine. On the other hand, the results also provide information about the modified and new implemented methods of treatment in most disciplines of the hospitals of the hospital association.

\section{KHT-P05 \\ Low titre nonspecific cold agglutinin antibodies causing blood clotting in hypothermic cardiopulmonary bypass: A case report}

\author{
${ }^{\star} N$. Sareban ${ }^{1}$, A. E. Yates ${ }^{2}$, S. Sipurzynski', S. Raile ${ }^{3}$, G. Lanzer ${ }^{1}$, \\ T. Wagner ${ }^{1}$
}

${ }^{1} \mathrm{LKH}$ Graz, Universitätsklinik für Blutgruppenserologie und

Transfusionsmedizin, Graz, Austria

${ }^{2}$ LKH Graz, Universitätsklinik für Chirurgie, Klinische Abteilung für Herzchirurgie, Graz, Austria

${ }^{3}$ LKH Graz, Universitätsklinik für Anästhesiologie und Intensivmedizin, Graz, Austria

Background: A 62 year old man was admitted to our hospital due to instable angina pectoris with massive retrosternal pain. A cardiac catheter was performed and complete obstruction of the LAD (left anterior descending) and the RCA (right coronary artery) was diagnosed. As catheter intervention was not possible, cardiac surgery with cardiopulmonary bypass (CPB) was planned. Standard CPB practice includes systemic cooling of the patient to $34{ }^{\circ} \mathrm{C}$ as well as intracoronary exposure to a cold cardioplegia solution (Buckberg).

Methods: Blood samples of cardiac surgery patients are generally screened for irregular antibodies using Coombs (IAT) and Bromelin. Additionally a direct antiglobulin test is performed. Cross-match for $6 \mathrm{RBC}$ units is done at $37^{\circ} \mathrm{C}$ in IAT. Preoperative cold agglutinin screening has not been performed so far.

Results: The antibody screening was found negative in IAT but positive in the one-stage enzyme-milieu. Subsequently, antibody differentiation was performed, but no specific antibody was detected. Interestingly, DCT was positive with $\mathrm{C} 3 \mathrm{~d}$ only and a titre of $1: 8$. Cross-match with the RBC units was negative.

In scope of the surgery the first application of the cold cardioplegia resulted in delayed blood clotting at the injection site, thus at the coldest exposure of the hypothermic CPB.

After being informed about the intraoperative adverse event, cold agglutinin antibodies were suspected and further tests were performed. Cold agglutinin antibodies were detected with a titre of $1: 32$ at $4{ }^{\circ} \mathrm{C}, 1: 16$ at 
$13{ }^{\circ} \mathrm{C}$ and 1:2 at room temperature and were nonspecific. The patient was warmed up immediately to withhold harmful events such as vascular occlusion.

Conclusion: Finally, the reason for the development of blood clots was low titre cold agglutinin antibodies with thermal range up to room temperature. As a consequence of this case perioperative procedures have been changed and an antibody screening test at $13{ }^{\circ} \mathrm{C}$ has been added. In the following 3 months two further patients were found with similar cold agglutinin antibodies and therefore surgery was performed without using cold cardioplegia. After one year of observation a re-evaluation will be conducted.

\section{KHT-P06}

\section{Continuous supply with extreme rare HPA-5bb platelet concentrates through a phase of MUD transplantation for a patient with clinical relevant HPA-5a antibodies}

\author{
H. Baume1, M.P. Ufen², A. Döscher', J. Casper², \\ ${ }^{\star}$ E.K. Petershofen ${ }^{1}$ \\ ${ }^{1}$ DRK Blutspendedienst NSTOB, Oldenburg, Oldenburg, Germany \\ ${ }^{2}$ Klinikum Oldenburg, Abt. Hämatologie, Oncologie \& Transplantation, \\ Oldenburg, Germany
}

Background: Patients with specific antibodies against human platelet antigens (HPA) need selected platelets in case of therapeutic treatment to prevent or reduce haemorrhagic symptoms. Patients with anti-HPA-5a need donors with HPA-5bb typing. Antigen frequency in Caucasians for HPA-5b is about $20 \%$ and for HPA-5a $99 \%$. In Germany six potential donors were available at that time.

Case history: A 68-year old woman was treated for secondary acute myeloid leukaemia (AML) following a myelodysplastic syndrome in 10/2010. Platelet count during AML therapy was refractory to transfusions with regular aphaeresis platelets. At this stage we detected a high titre anti-HPA-5a antibody (dilution 1/2048) in the patient's serum. HPA-5bb (5a-negative) platelet concentrates had to be produced to gain satisfactory platelet count increments during the next months. In February 2011 it was decided to use allogeneic bone marrow transplantation (aBMT) for further therapy. As a first step an antibody immunoadsorption was planned. Prior to allogeneic blood stem cell transplantation the patient was treated once with Rituximab. However, 4 weeks ahead of transplantation the HPA-5a antibody had fallen to such a low titre that immunoadsorption was not affordable.

In June 2011 the patient received a matched unrelated donor transplantation (10/10; peripheral blood stem cells) from a person being HPA-5a antigen positive. On day +28 the patient had trilineage engraftment and in the following weeks no persistent thrombocytopenia. Between July and November 2011 the anti-HPA-5a antibody was not detectable.

Methods \& Results: Pre-Tx screening for anti-HLA was negative and for anti-HPA positive. An inhouse MAIPA assay showed specific reactions for anti-HPA 5a in combination with HPA-5a-positive reference cells. Pre-Tx patient typing: positive for HPA5b; negative for HPA5a. Total transfusions (2-4.5x10E11 PLT per single donor unit): unselected firstline therapy $=10$; selected during $\mathrm{AML}$ therapy $=14$; during mud-transplantation $=11$.

Conclusion: The strong HPA-5a antibody titre decreased spontaneously over a period of 7 months using compatible PLT. For Rituximab, it is unlikely to cause a rapid clearance of HPA-antibodies, however Rituximab may have prevented the reappearance of the antibody after transplantation when HPA-5a-positive donor derived platelets were produced in the patient. Although HPA-5bb donors are extremely rare in Germany, a sufficient PLT supply could be organized due to a fine tuned preorganization between German PLT-centres.

\section{KHT-P07}

\section{Management of severe bleeding anemia in a patient with high risk of alloimmunisation}

${ }^{*}$ N. Ostendorf', J. Zeeh ${ }^{2}$

${ }^{1}$ St. Katharinen-Hospital, Zentrallabor, Frechen, Germany

${ }^{2}$ St. Katharinen-Hospital, Gastroenterologie, Frechen, Germany

Background: In patients with certain rare blood group constellations, it is virtually impossible to find compatible red cell concentrates, once they are immunised against common antigens. In case of a life-threatening bleeding-episode, the risk of anemia has to be weighted against the risk of immunisation by transfusion.

Methods: We discuss the case of a woman with the extremely rare Rhesus-phenotype CCD.EE. The frequency of this phenotype in Europe is estimated below 1 in 10.000 . The patient is of indonesian offspring. She had hemoglobin concentrations as low as $4.6 \mathrm{~g} / \mathrm{dl}$ due to chronic bleeding from a duodenal ulcer and severe iron deficiency. No compatible red cell concentrates could be obtained from any transfusion service in the whole region.

Results: Incompatible red cell transfusion was avoided by closely surveying the patient and by supplementing iron intravenously for 8 days. The resolution of iron deficiency was monitored effectively by the mean reticulocyte hemoglobin content, a parameter widely available on current hematology analysers. If a transfusion had been necessary, a scheme was introduced to use only red cells of Rh-phenotype CCD.ee, to maintain a second chance in the future.

Conclusion: To correctly communicate and assess the risk of anemia vs. transfusion with prospective immunisation, good collaboration between blood bank and clinical medicine is needed. The patient was instructed to seek autologous blood donation in case of elective surgery.

\section{KHT-P08 \\ Lyophilized plasma and fresh frozen plasma are equally effective in correcting impaired plasmatic coagulation}

\section{${ }^{*} U$. Sachs ${ }^{1}$, R. Marschall ${ }^{2}$, G. Dinges ${ }^{3}$}

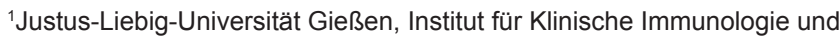
Transfusionsmedizin, Gießen, Germany

Universitätsklinikum Gießen und Marburg, Zentrum für

Transfusionsmedizin, Marburg, Germany

${ }^{3}$ Universitätsklinikum Gießen und Marburg, Klinik für Anästhesiologie und Intensivtherapie, Marburg, Germany

Background: In massive blood loss, transfusion of blood components remains the mainstay of treatment, with some recent studies advocating an early and more aggressive use of plasma. In contrast to fresh frozen plasma (FFP), lyophilised (freeze-dried) plasma (LP) can be stored at room temperature and reconstitution for transfusion is faster than thawing FFP. However, only few data regarding the efficacy of LP are available in literature.

Methods: Citrated blood samples from 150 consecutive patients with prolonged prothrombin time $(\mathrm{TP}>16.4 \mathrm{sec}=\mathrm{INR}>1.5)$ were stored at $-70{ }^{\circ} \mathrm{C}$ until use. After thawing in a water bath at $37{ }^{\circ} \mathrm{C}$, samples were split and mixed in a 4:1 ratio with freshly thawed FFP or reconstituted LP, mimicking a transfusion of $\sim 20 \mathrm{ml} / \mathrm{kg}$ body weight. Aliquots from each FFP $(n=50)$ and LP $(n=50)$ were used for three random patient samples. All samples were assessed for INR, protein $\mathrm{S}$, and coagulation factor $(\mathrm{F})$ $\mathrm{V}$ and VII activity, and mixed samples were also assessed for fibrinogen concentration using standard assays on a BCS-XP device (Siemens Health Care Diagnostics, Eschborn, Germany). Statistics were performed by Mann Whitney U test.

Results: The following mean values were obtained in the patient samples: INR 2.7 (range, 1.5 to 5.9), protein S 50\% (range, 2.7 to 122.8), FV activity $87.7 \%$ (range, 51 to 143 ), and FVII activity $33 \%$ (range, 7 to 78 ). After addition of FFP, the mean values were INR 1.6 (1.3 to 2.3), fibrinogen $3.8 \mathrm{~g} / \mathrm{L}$ (1.4 to 8.1), protein S 60\% (24 to 107$)$, FV 91\% (62 to 147 ), and FVII $49 \%$ (24 to 83 ). After addition of LP, the mean values were INR 
1.5 (1.2 to 2.1 ), fibrinogen $3.8 \mathrm{~g} / \mathrm{L}$ (1.8 to 8.1 ), protein $\mathrm{S} 62 \%$ (30 to 113 ), FV $92 \%$ (69 to 139), and FVII 53\% (23 to 87). There were no statistical differences for INR $(p=0.35)$, fibrinogen $(p=0.92)$, protein $S(p=0.09)$, FV $(p=0.46)$, or FVII $(p=0.22)$ between the two groups.

Conclusion: This study demonstrates that lyophilised plasma is comparable to fresh frozen plasma in correcting impaired plasmatic coagulation in vitro. Since lyophilised plasma can be stored at room temperature and can be reconstituted rapidly, it is an attractive alternative to FFP, especially in emergency settings. In vivo studies are required to prove whether this is advantageous for our patients.

\section{KHT-P09}

\section{Severe hemolytic anemia after treatment with intravenous immunoglobulin}

*U.F. Königbauer', F. Seidel², S. Teller², H. Jahn-Jochem', R. Offner ${ }^{2}$, F. Weinauer ${ }^{3}$

${ }^{1}$ Blutspendedienst des BRK, Institut für Tranfusionsmedizin, Nürnberg, Germany

${ }^{2}$ Klinikum Bayreuth, Bayreuth, Germany

${ }^{3}$ Blutspendedienst des BRK, Institut für Tranfusionsmedizin, München, Germany

Background: Hemolytic anemia after high-dose (1-2 g/kg body weight) intravenous immunoglobulin (IVIG) therapy due to blood group isoagglutinins is a known, but rare complication in non-0 blood group recipients. Only one IVIG preparation $\left(\right.$ Privigen $^{\circledR}$ ) contains an additional warning in its package insert related to the risk of hemolysis. According to the European Pharmacopoeia, anti-A and anti-B titers in IVIG 5\% must not exceed 64. We report a case of severe hemolysis after receiving IVIG containing high isoagglutinin titers.

Case report: A 60-year-old woman received IVIG $\left(10 \%\right.$ Octagam $\left.^{\circledR}\right) 30 \mathrm{~g} /$ day $(0.4 \mathrm{~g} / \mathrm{kg} / \mathrm{d})$ for four consecutive days for possible myasthenia gravis prior to confirmation of food-borne botulism. Her hemoglobin, bilirubin and LDH were normal prior to IVIG therapy. Brown urine was noticed on the third treatment day. One day after her last dose her bilirubin rose to 7.0 $\mathrm{mg} / \mathrm{dl}$ accompanied by a rising LDH up to $1970 \mathrm{U} / 1$, low haptoglobin $(<7$ $\mathrm{mg} / \mathrm{dl}$ ), and a drop in hemoglobin ( $\mathrm{Hb})$ consistent with hemolytic anemia. During the night red cells for transfusion were ordered. The following day the patient needed emergent thrombectomy for phlegmasia cerulea dolens. Pre-operative $\mathrm{Hb}$ was $5.1 \mathrm{~g} / \mathrm{dl}$. After intraoperative transfusion of four units of blood group 0 red cells her hemoglobin level remained stable at 9 to $10 \mathrm{~g} / \mathrm{dl}$ and bilirubin and LDH gradually declined. Renal function remained normal.

Results: The patient's red cells typed A1B, $\mathrm{Rh}(\mathrm{D})$ negative. Her reverse type, however, revealed anti-A1 and anti-B. Her DAT was reactive with anti-IgG and the eluate was positive with anti-A1, anti-A2 and anti-B. Compatibility testing was positive with blood group $\mathrm{AB}$ and negative with blood group 0 red cells for transfusion. Isoagglutinin titers of the same lot of Octagam ${ }^{\circledR}$ the patient had received were 1024 for anti-A1 and 256 for anti-A2 as well as anti-B, performed on anti-IgG/C3d microtube columns at $37^{\circ} \mathrm{C}$.

Conclusion: A four-day treatment with IVIG containing high levels of isoagglutinins resulted in severe hemolytic anemia with a drop in hemoglobin of more than $6 \mathrm{~g} / \mathrm{dl}$ in a blood group A1B patient. To reduce the risk of this complication, parameters for hemolysis should closely be monitored in non-0 blood group patients receiving IVIG. Additional warnings in patient information leaflets should be considered for all IVIG preparations to heighten the awareness in clinicians and ensure the possibility for early interventions.

\section{KHT-P10 \\ Quantity of RBC concentrates and defined safety level of blood-supply for operative procedures}

\section{${ }^{*}$ C. Hagen ${ }^{1}$, R. Richter ${ }^{2}$, H. Reißmann 2 , S. Görg', C. Brockmann ${ }^{1}$}

${ }^{1} \mathrm{UKSH}$, Inst. für Transfusionsmedizin, Lübeck, Germany

2UKSH, Stabsstelle Medizinischer Sachbedarf, Lübeck, Germany

Background: According to the German Guidelines for haemotherapy patients have to be informed about the possibility of autologous blood donation if the transfusion probability exceeds $10 \%$ and necessary blood tests incl. cross-matches have to be ordered. The "regular requirement" of RBC-concentrates (RBC) for different operative procedures has to be determined based on in-house consumption data. However, there is so far no generally accepted definition of a "regular requirement".

Methods: With a retrospective analysis of in-house data of the year 2011, we have evaluated the documented quantity of RBC related to selected operative procedures, according to the OPS-Code, an adaption of the ICPM. In cases with more than one operative procedure, we defined main procedures and matched these in a database query with the quantity of documented transfusions. We determined the present transfusion probability and statistical values like median, average quantity of transfused RBC and the most frequent count of transfused units.

Results: The following examples represent the transfusion probability, the arithmetic average of quantity, the median and the number of RBC ensuring a supply safety in $95 \%$ of all cases.

\begin{tabular}{|l|l|l|l|l|l|}
\hline Operative procedure & $\begin{array}{l}\text { Number } \\
\text { of cases }\end{array}$ & $\begin{array}{l}\text { Transfusion } \\
\text { probability }\end{array}$ & $\begin{array}{l}\text { Average } \\
\text { RBC }\end{array}$ & $\begin{array}{l}\text { Median } \\
\text { RBC }\end{array}$ & $\begin{array}{l}\text { Number } \\
\text { of RBC } \\
\text { for a 95\% } \\
\text { safety of } \\
\text { supply }\end{array}$ \\
\hline Liver transplantation & 50 & $86 \%$ & 9 & 7.5 & 29 \\
\hline Total gastrectomy & 38 & $37 \%$ & 1.6 & 0 & 7 \\
\hline Total hip replacement & 511 & $13 \%$ & 0.4 & 0 & 2 \\
\hline Aortocoronary bypass & 1578 & $65 \%$ & 2.5 & 2 & 8 \\
\hline Aortic aneurysm & 189 & $47 \%$ & 2.8 & 0 & 12 \\
\hline $\begin{array}{l}\text { Aortic valve } \\
\text { replacement }\end{array}$ & 874 & $60 \%$ & 2.4 & 2 & 8 \\
\hline
\end{tabular}

The arithmetic average of the number of transfused RBC is not suitable to calculate a "regular requirement", because this value is influenced by statistical outliers. Similarly the median is not suitable for calculation, because the level of safety is variable from $50 \%$ to theoretically $100 \%$.

Conclusion: The present local recommendations based on estimates of clinicians achieve for many procedures a very high safety level of supply of nearly $95 \%$. However, from an economic point of view a high quantitative safety-level means many cross-matches without real consumption. It should be discussed, if the reservation of high amounts of RBC for 3 days leads to an increased expiration of blood products.

\section{KHT-P11 \\ Experience with a Massive Transfusion Protocol in the Trauma Setting from the Blood Bank Perspective}

\section{${ }^{\star}$ R. Conradi', S. Kuhn'2, G. Maccagno', * D. Marandiuc ${ }^{1}$,} W.E. Hitzler ${ }^{1}$

${ }^{1}$ Universitätsmedizin Mainz, Transfusionszentrale, Mainz, Germany ${ }^{2}$ Universitätsmedizin Mainz, Klinik und Poliklinik für Unfallchirurgie, Mainz, Germany

Background: In the trauma setting the implementation of a massive transfusion protocol (MTP) using a balanced ratio of plasma and platelets to red blood cells is recommended to improve patient outcome. Since April 2011 the physician on duty at our emergency department activates a MTP as soon as he has been notified from the pre-hospital care that a patient with a major trauma and specific (in the protocol defined) injury patterns is being transported to our hospital. Such an early activation is necessary because of a distance of $0.5 \mathrm{Km}$ between emergency department and blood bank. 
Methods: According to our protocol blood components are issued in packages: the first package contains 6 red blood cell concentrates (RBC) of the $\mathrm{O}$ blood group and 4 fresh frozen plasma (FFP) of the $\mathrm{AB}$ blood group, the second and the following packages contain additionally 2 apheresis platelet concentrates (PLT) each. Packages for children up to the age of 14 years contain half the amount of blood components. If bleeding continues, at the beginning of the transfusion of the third $\mathrm{RBC}$ in adult patients (of the second in children) a further package should be requested. Our transfusion goal is to maintain a $\mathrm{Hb}$ level of $10 \mathrm{~g} / \mathrm{dl}$ and a platelet count $>100.000 / \mu 1$. The aim of this analysis was to define the frequency of massive transfusions in trauma patients in calendar Year 2012 , to assess how the requested blood components are utilized, and to quantify the related wastage of blood products.

Results: A MTP was activated for 69 patients. Among them $35(50.7 \%)$ received no transfusion, $3(4.3 \%)$ received only FFP, $7(10.1 \%)$ only RBC, 17 (24.6\%) RBC and FFP, 6 (8.7\%) RBC, FFP and PLT. Only 6 patients $(8.7 \%)$ received a massive transfusion $(>10 \mathrm{RBC})$, and particularly $15.8 \pm 4.0(11-22) \mathrm{RBC}-$ mean $\pm \mathrm{SD}$ (range) - $11.3 \pm 2.7(8-16)$ FFP and $2.3 \pm 1.2$ (1-4) PLT. For all patients 507 RBC, 338 FFP and 32 PLT were requested, 202 RBC, 144 FFP and 14 PLT were transfused, 305 RBC, 194 FFP and 18 PLT were sent back to the blood bank. $41 \%$ of the issued blood products were transfused (details are presented in the table) Among the returned blood components all FFP, $8(2.6 \%)$ RBC and 4 (22.2\%) PLT had to be discarded because they had not been properly stored.

Conclusion: Our main finding is that only a small amount of patients received a massive transfusion. Half of the patients did not even receive a blood transfusion, so that many blood products were returned to the blood bank with a high rate of wastage particularly for FFP. As a possible reason physicians in the pre-hospital care tend to overestimate the patient's degree of injury severity. An improvement of the decision process to initiate a MTP would reduce wastage of blood products and costs. Reviewing the basis for that process would be an important quality measure for our clinicians.

\begin{tabular}{|c|c|c|c|c|}
\hline \multirow{2}{*}{$\begin{array}{c}\text { Number of } \\
\text { requested } \\
\text { packages }\end{array}$} & \multirow{2}{*}{$\begin{array}{c}\text { Number of } \\
\text { patients }\end{array}$} & \multicolumn{3}{|c|}{ Percentage of transfused products } \\
\cline { 3 - 5 } & 59 & RBC & FFP & PLT \\
\hline 1 & 5 & $62 \%$ & $26 \%$ & $0 \%$ \\
\hline $1+2$ & 4 & $86 \%$ & $60 \%$ & $20 \%$ \\
\hline $1+2+3$ & 1 & $92 \%$ & $100 \%$ & $50 \%$ \\
\hline $1+2+3+4$ & 5 & $67 \%$ \\
\hline
\end{tabular}

Table: Number of patients who received $1,2,3$ or 4 packages and ratio of transfused blood-products.

\section{KHT-P12}

Influence of hemoglobin concentration on intensive care stay: A retrospective analysis in 348 consecutive elective coronary bypass patients

\author{
*J. Cheko, J. Albers, C. F. Vahl \\ Unimedizin Mainz, HTG, Mainz, Germany
}

Background: Optimal target hemoglobin levels $(\mathrm{Hb})$ in coronary bypass patients are being discussed controversially. We therefore investigated if the perioperative $\mathrm{Hb}$ in elective coronary surgery had an influence on the length of stay on the intensive care unit (ICU).

Methods: In a retrospective study 348 consecutive patients undergoing elective coronary surgery in 2012 were analyzed for the following questions: Do the preoperative $\mathrm{Hb}$ upon hospital admission $(\mathrm{A}-\mathrm{Hb})$ and/or the postoperative $\mathrm{Hb}$ upon ICU admission $(\mathrm{Op}-\mathrm{Hb})$ have a significant influence on the postoperative course (length of stay on ICU, rethoracotomy because of bleeding)? Statistical analysis (SPSS 21) was performed with ANOVA and correlation analysis (Pearson).

Results: Mean A-Hb was $13.5 \pm 1.70 \mathrm{mg} / \mathrm{dl}$; mean $\mathrm{Op}-\mathrm{Hb} 11.01 \pm 1.01$ $\mathrm{mg} / \mathrm{dl}$. A-Hb did not show an influence on ICU length of stay $(\mathrm{p}=0.938)$. For Op-Hb, however, a correlation could be found $\left(\mathrm{R}^{2}=0,04 ; \mathrm{p}=0,044\right)$. A higher Op-Hb correlated with a significantly shorter ICU stay. Neither $\mathrm{A}-\mathrm{Hb}(\mathrm{p}=0.30)$ nor $\mathrm{Op}-\mathrm{Hb}(\mathrm{p}=0.73)$ influenced the rate of rethoracotomies in our patients.

Conclusion: It is interesting that neither $\mathrm{A}-\mathrm{Hb}$ nor $\mathrm{Op}-\mathrm{Hb}$ influenced the rate of rethoracotomies in our electively operated patients. A higher $\mathrm{Op}-\mathrm{Hb}$, however, was associated with a shorter ICU stay. Demographic development will lead to an increase in elderly patients with relative anemia. This should not necessarily be related with a worse postoperative course in elective coronary bypass patients.

\section{KHT-P13}

\section{Implementation of Standard Operating Procedures (SOPs) for transfusion triggers reduces intraoperative transfusion requirements in elective cardiac-surgery}

\section{${ }^{*}$ C. Rosenthal, J. Wicht, M. Sander, U. Knipprath,} C. von Heymann

Charité Universitätsmedizin Berlin, Klinik für Anästhesiologie m.S. operative Intensivmedizin Campus Virchow-Klinikum, Berlin, Germany

Background: Irrespective of blood supply shortages and costs of blood products, transfusion of even small amounts of blood products is associated with increased mortality and morbidity rates in cardiac surgery patients. Even though guidelines for perioperative blood conservation in cardiac-surgery exist, implementation rate in clinical practice is low. For these reasons it is important to study measures for guideline implementation in daily clinical practice. In this prospective single-centre study, we investigated the incidence of blood product transfusion in cardiac surgery patients before and after implementation of a transfusion SOP.

Methods: Transfusion behaviour was prospectively studied in elective adult cardiac surgery patients on four consecutive weeks before and eight consecutive weeks after implementation of a SOP on transfusion practice. Beside recording the amount of blood products transfused, the anesthesiologists were asked to state on a separate questionnaire the indication for every blood-product (e.g. pre transfusion hemoglobin, prolonged coagulation times etc.).

Results: Overall 181 patients were included in this study, 49 before and 132 after SOP implementation. Patients with emergency-surgery and with mass transfusion were excluded from analysis. 42 patients before and 110 patients after SOP-Implementation remained in this analysis. The two groups did not differ in age, concomitant diseases, ASA-status and Euro-Score. After SOP-implementation the number of transfused patients decreased significantly from $40,5 \%$ to $18,2 \%(p<0,05)$. En detail, transfusion requirements for erythrocytes dropped from $26,2 \%$ to $10,9 \%$ of patients ( $\mathrm{p}$

Conclusion: The implementation of a Guideline-based Standard-operating procedure on transfusion triggers and practice resulted in a significant reduction of transfusion requirements in elective cardiac surgery patients. 


\section{Workshop: Neue Trends in der molekularen Diagnostik}

\section{NTM-V01}

Phäno- und Genotypisierung in der Molekularen Diagnostik

\section{Neumaier}

Universitätsmedizin Mannheim (UMM) , Institut für klinische Chemie, Mannheim, Germany

\section{NTM-V02}

\section{Genotyping of Blood Group Antigens}

\section{Gassner}

Blutspende Zürich SRK, Schlieren, Switzerland

\section{NTM-V03}

\section{Biobanking}

${ }^{*}$ T. Illig

Medizinische Hochschule Hannover (MHH), Hannover, Germany

\section{NTM-V04}

Analysis of extracellular vesicles (EVs) in diagnostics and quality control of blood products in the transfusion medicine

\section{${ }^{*}$ G. Schmitz', A. Black ${ }^{1}$, A. Böttcher ${ }^{1}$, A. Pienimäki-Römer ${ }^{1}$, K. Kuhlmann', G. Liebisch', T. Konovalova', H. E. Meyer', E. Orsó ${ }^{1}$}

${ }^{1}$ University Hospital Regensburg, Institute for Laboratory Medicine and Transfusion Medicine, Regensburg, Germany

${ }^{2}$ Ruhr-University Bochum, Department of Medical Proteomics, Medical Proteomic Center, Bochum, Germany

Background: Extracellular vesicles (EVs) are cell-derived secretory and apoptotic vesicular structures, shed into the extracellular milieu. Recently, EV research rapidly expands due to available novel technologies and biomarkers for their analysis.

Methods: Three types of human EVs have been characterized by multi-omics analysis: (i) five subsets (F1-F5) of platelet-derived EVs $(P L-E V S)$ isolated by density gradient centrifugation from platelet concentrates (PCs) during platelet senescence, (ii) erythrocyte-derived EVs $(R B C-E V s)$ in erythrocyte concentrates (ECs) and PCs, and (iii) neutrophil-derived EVs ( $P M N-E V s)$ of isolated cells, exposed to atherogenic lipoproteins or inflammatory stimuli.

Methods: The EVs were analyzed by nanoparticle tracking analysis, advanced high sensitivity flow cytometry (Apogee 50M), mass spectrometry-based lipidomics and proteomics and microarrays for micro-RNA (miRNA) expression.

Results: PL-EVs represent the major part of all EVs in PCs, ECs and plasma. The subsets F1-F2 have the highest content of ESCRTs, RNA-binding proteins, CD62P, the Parkinson's disease (PD)-related alpha-synuclein (aSyn), and free cholesterol (FC). Major biomarkers in F2-F4 include CD63, CD107a, the Alzheimer's disease (AD)-associated amyloid-precursor protein (APP) and 7-ketocholesterol. Minor lipid classes, such as lysophosphatidic acid, phosphatidylserine, ceramide, phosphatidylglycerol and cardiolipin, predominate in F5. The subsets F3-F5 are enriched in caveolin-1 and apolipoproteins, whereas F5 accumulate mitochondrial proteins. PL-EVs increase during in vitroplatelet senescence, parallel with enrichment of AD- and PD-related miRNAs.
Conclusion: In comparison to PL-EVs, RBC-EVs are particularly enriched in aSyn, sphingomyelin and FC, parallel with diminished content phosphatidylcholine and plasmalogens.

PMN-EVs dramatically shed in response to atherogenic lipoproteins and LPS. PMN-EVs are chemotactic for monocytes and induce apoptosis/necrosis, prior to splenic clearance or entering vascular lesions.

Conclusion: Qualitative and quantitative changes in blood cell-derived EVs are paralleled with disease pathologies, and their biomarkers may reflect the quality and senescence of hemotherapeutic products and open up new avenues towards EV-apheresis therapy.

\section{Workshop: Neue Trends in der Transplantationsmedizin}

\author{
NTT-V01 \\ Principle and Application of Immunoadsorption \\ N. Braun \\ MediClin Müritz-Klinikum, Waren, Germany
}

Although plasmapheresis still is the prevailing apheresis technique, immunoadsorption gains more and more acceptance for a wide field of clinical applications. Immunoadsorption techniques can be subdivided into three major classes: Non-selective, semi-selective and highly selective, based on the quantity and purity grade of adsorbed material from blood stream. Up to now, most clinical immunoadsorption techniques requires a two step procedure: plasma separation by either membrane plasmapheresis or centrifugation followed by the adsorption onto a specified adsorber material.

Immunoadsorption allows elimination of soluble immunoglobulines and immune complexes below detection limit due to its high efficacy while conventional plasmapheresis is limited to a clearance rate of approximately $60 \%$ per processed relative plasma volume. However, selective immunoadsorption may not always be clinically effective because many immunologically mediated diseases are caused by complex underlying immune mechanisms not limited to a specified antibody-antigen interaction. Understanding pathomechanisms and selection of the appropriate apheresis technique is mandatory for successful treatment.

\section{NTT-V02 \\ Therapeutic apheresis in cardiac and lung transplant patients}

\section{${ }^{*}$ S. Rummler ${ }^{1}$, K. Maier ${ }^{1}$, T. Steinke ${ }^{2}$, D. Barz}

${ }^{1}$ Universitätsklinikum Jena, Institut für Transfusionsmedizin, Jena, Germany

${ }^{2}$ Universitätsklinikum Jena, Klinik für Herz- und Thoraxchirurgie , Jena, Germany

Background: Immunological problems like preformed donor specific antibodies (DSA) or high degree of immunization, complicate the transplantation (TX) and can limit the therapeutic success. Dyslipaemic patients or patients with prolonged cellular rejection episodes are also at high risk. Essential pillars of antihumoral therapy are extracorporeal procedures like therapeutic plasma exchange (TPE), high plasma volume immunoadsorption (IA) and photochemotherapy (ECP).

Methods: In the last 5 years a total of 27 patients, 12 heart transplant (HTX) patients, 3 patients after combined lung and heart transplantation $(\mathrm{Lu} / \mathrm{HTX})$ and 12 lung transplant (LuTX) patients were treated for prevention (7 patients) or in case of rejection (20 patients) with TPE, cascade filtration (CF), IA or ECP.

Results: 15 transplant recipients were tested positive for HLA-ab either donor specific or de novo 
HLA-ab. Altogether 7 patients were tested positive for non-HLA-ab. 6 patients were treated prior to TX because of pre-existing antibodies. After TX we treated 24 patients initially with TPE and performed on average 4 TPEs (1-11) for antibody elimination. In 6 cases we switched the therapy from TPE to IA or CF and 10 patients were treated further with ECP. 22 of 27 treated patients survived with stable graft function.

Conclusion: The extracorporeal procedures we performed are qualified for rescue therapy of hyper acute rejection episodes or to prevent acute rejection episodes.

\section{NTT-V03}

Efficacy of plasma exchange in the treatment of antibodymediated rejection in renal transplantation

\section{Busch}

Klinik für Innere Medizin III, Nephrologie, Jena, Germany

Background: Antibody-mediated rejections (ABMR) are detrimental to transplant survival. HLA-antibodies of various clinical relevance are present in up to $30 \%$ of renal transplant recipients. New methods of antibody detection contribute to a more sensitive identification of donor-specific antibodies (DSA). The new onset of DSA is associated with a graft survival of at most $50 \%$ at 5 years.

Methods: Plasmapheresis (PPH) and/ or administration of intravenous immunoglobins (IVIG) are established methods in the treatment of ABMR. The B-cell depleting antibody rituximab (RTX) as well as the proteasome-inhibitor bortezomib which induces apoptosis of plasma cells are newer drug strategies against ABMR. Since these forms of therapy influence mainly the formation of antibodies, they must be combined with antibody-removing therapies such as plasmapheresis or immunoadsorption to remove already existing antibodies.

Results: PPH is a fast and widely available method. By its use, severe antibody deficiency states can be avoided. Complement is removed from the peripheral blood if plasma is replaced by electrolyte solution and albumin Complement factor C3 reduction rates of up to $85 \%$ were described. In ABMR, many studies and case series, usually with small numbers of cases suggest benefit of PPH if combined with IVIG or RTX. However, the evidence belonging different therapeutic schemes using PPH is limited.

Conclusion: Larger randomized controlled studies on the treatment of ABMR are therefore urgently needed. The prognosis of ABMR is still unsatisfactory.

\section{NTT-V04}

\section{Capability for antibody detection in eluates obtained by immunoadsorption (IA)}

\section{${ }^{*}$ D. Barz, S. Friedrich, S. Rummler}

Universitätsklinikum Jena, Institut für Transfusionsmedizin, Jena, Germany

Background: Vascular rejection is the B-cell-mediated production of immunoglobulin $\mathrm{G}(\mathrm{IgG})$ antibodies $(\mathrm{AB})$ against the transplanted organ. The quick removal of $\mathrm{AB}$ and other plasma factors via IA remains an effective and supportive method for treating AMR, but there is no satisfactory answer when, how often and for how long treatment should be administered. Is it possible that the behaviour of the $\mathrm{AB}$ concentration in eluates from protein A sepharose column can give an answer?

Methods: A total of 13 transplant recipients ( 9 kidneys and 4 hearts) suffering from AMR were analysed. In total patients received 73 high-volume IA (Immunosorba, Fresenius Medical,Bad Homburg,Germany). In order to overcome AMR 3 to 25 treatments per patient were performed. HLA- and non-HLA-AB were tested in sera before and after IA as well as in eluates. Eluates are defined as solution resulting from elution of protein A sepharose columns and neutralised with TRIS-buffer.

Following methods were used for the detection of HLA- and non-HLAAB: Complement dependent lymphocytotoxicity test (CDC), Solid-phase enzyme-linked immunosorbent assay (ELISA), Luminex for HLA-AB and ELISA for anti-endothelial cell antibodies (AECA) like Angiotensin II type 1 receptor (AT1), endothelin-1 type A receptor (ETA) and glycoprotein (GP) IIb/IIIa, Ib/IX and Ia/IIa.

Results: Before IA treatment HLA-AB (CDC) in sera were detected in $27 \%$ versus $39 \%$ in eluates and $46 \%$ versus $87 \%$ by using ELISA. We could not find any AB against GP in sera. In eluates, however, we could detect AB against GP: GP IIb/IIIa in $86 \%$ of all samples with titres from $1: 1$ to $1: 32$, GP Ib/IX (up to $1: 32$ ) in $76 \%$ and GP Ia/IIa with titres from $1: 1$ to $1: 16$ in $82 \%$. Further we detected AECA against receptors AT1 and ETA in sera before IA in $22 \%$, after IA in $10 \%$ and in eluates in $42 \%$ of all samples. The antibody titres vary from 1:1 to $1: 256$.

Conclusion: Our investigation pointed out, that AMR is still possible without detectable $\mathrm{AB}$ in serum and consolidates the hypothesis that clinical relevant non-HLA-AB and HLA-AB are partly fixed on the graft.High-volumeIAis qualified to detach fixed $A B$. If this hypothesis is true, the eluate as a medium for detection of these $\mathrm{AB}$ is more suitable than sera.

\section{Oral Abstract Session: Stammzelltransplantation \& Stammzellbiologie}

\section{SZT-V01}

Successful treatment of therapy-refractory acute Graftversus-Host Disease with mesenchymal stem cell-derived exosomes

\section{Kordelas ${ }^{\text {, , V. Rebmann }}$, A.-K. Ludwig ${ }^{2}$, S. Radtke ${ }^{2}$, P.A. Horn², D. Beelen ${ }^{1},{ }^{*} B$. Giebel ${ }^{2}$}

${ }^{1}$ Department of Bone Marrow Transplantation, University Hospital Essen, Essen, Germany

${ }^{2}$ Institute for Transfusion Medicine, University Hospital Essen, Essen, Germany

Background: A number of studies reported positive impacts of systemically applied mesenchymal stem cells (MSCs) for preventing or treating acute Graft-versus-Host Disease (GvHD). In contrast to the initial paradigm that MSCs intercalate into injured tissues and thus reduce tissue damage, it is now widely assumed that MSCs secrete a number of immune-modulatory factors, which impair inflammation and thus help to suppress GvHD. Exosomes are secreted cell organelles, which exert immune-modulatory properties. These small membrane vesicles are released by a huge variety of different cell species, including MSCs.

Methods: Aiming to treat a 22-years female patient suffering from severe therapy-refractory cutaneous and intestinal GvHD grade IV with MSC-derived exosomes, exosomes from bone marrow derived MSCs of an unrelated stem cell donor were enriched. The exosome preparation's immune modulatory properties were confirmed in vitro, before exosomes were administered in escalating doses to the patient. Impacts on the clinical symptoms and the immune response were documented

Results: Administration of the exosome preparation to the GvHD patient was well tolerated. The cutaneous and intestinal GvHD symptoms improved significantly and the dosage of immunosuppressive agents - particularly of steroids - could be reduced. In line with this, a reduction of the pro-inflammatory cytokine response was observed during the course of the therapy.

Conclusion: MSC exosomes exert immune suppressive functions in vitro and apparently help to suppress GvHD symptoms in vivo. Since the in vivo administration seems to be safe, MSC exosome administration appears to be a promising new treatment option for therapy-refractory GvHD. 
SZT-V02

\section{Inhibition of CD39 in human bone marrow mesenchymal stromal cells reduces colony formation, differentiation potential and immunosuppression}

${ }^{*}$ A. V. Gouveia de Andrade ${ }^{1,2}$, R. Wehner ${ }^{3}$, J. Karbanová4, D. Corbeil $^{4}$, J. Bastid ${ }^{5}$, M. Schmitz ${ }^{3,2}$, M. Odendahl',

\section{Bornhäuser ${ }^{2,6}$, T. Tonn ${ }^{1,2,7}$}

${ }^{1}$ German Red Cross Blood Donor Service North-East, Experimental Transfusion Medicine Laboratory, Dresden, Germany

${ }^{2}$ Center of Regenerative Therapies Dresden, Dresden, Germany

IInstitute of Immunology, Medical Faculty, Technical University of Dresden,

Dresden, Germany

${ }^{4}$ Tissue Engeneering Laboratories (BIOTEC), Technical University of Dresden, Dresden, Germany

${ }^{5}$ OREGA BIOTECH, , Ecully, France

${ }^{6}$ Department of Medicine I, University Hospital of Dresden, Dresden,

Germany

${ }^{7}$ Experimental Transfusion Medicine, Medical faculty, technical University of Dresden, Dresden, Germany

Background: Adoptive transfer of human bone marrow mesenchymal stromal cells (BM-MSCs) in the context of post-transplant GvHD relies on their immunosuppressive abilities, which are still not fully characterized. Recently, an important role for the CD39-CD73-adenosine pathway in mediating immunosuppression was demonstrated in Tregs. Since BM-MSCs express CD73 and recent data indicate that BM-MSCs express CD39, we decided to further investigate the CD39 expression in BM-MSCs and check whether inhibition of CD39 would impact not only on immunosuppressive capacity, but also on colony forming ability and differentiation potential.

Methods: Expression of CD39 in BM-MSC lines was assessed by flow cytometry, immunocytochemistry and western blotting. With the aim to check whether CD39 would influence immunosuppressive properties of MSCs, anti-CD39 monoclonal antibody OREG-103/BY40 (CD39mAb) and a highly potent chemical inhibitor of CD39, POM-1, were used within standardized immunosuppressive assays, such as the lymphocyte proliferation assay (LPA) and mixed lymphocyte reaction (MLR). For the colony forming unit (CFU-F) capacity, and in vitro differentiation potential into osteoblasts and adipocytes, POM-1 was used in 2 different concentrations ( 2 and $20 \mu \mathrm{M})$.

Results: All tested BM-MSC lines $(\mathrm{n}=20)$ expressed CD39 $(19.2 \pm 3.3 \%$ CD39+ cells among BM-MSCs). Flow cytometric results were confirmed by immunocytochemistry as well as western blotting. Notably, addition of POM-1 significantly impaired the immunosuppressive capacity of BM-MSCs (at the concentration of $20 \mu \mathrm{M}$ for the LPA and $2 \mu \mathrm{M}$ for the MLR) in both assays ( $n=6$, mean inhibition (MI) of $24.28 \pm 8.3 \%$ for the LPA; $n=4$, MI of $42.28 \pm 9.33 \%$ for the MLR) as compared to the non-treated BM-MSCs (MI of $70.2 \pm 2.7 \%$ for the LPA, $p<0.0001$; MI of $78.1 \pm 3.11 \%$ for the MLR, $\mathrm{p}<0.01)$. Preliminary results using the $\mathrm{CD} 39 \mathrm{mAb}$ indicate that the immunosuppressive capacity of BM-MSCs is reduced, confirming our results with POM-1. Furthermore, inhibition of CD39 by addition of POM-1 to the cultures hampered the colony formation ability of BM-MSCs $(n=3)$. Interestingly, we also demonstrated that POM-1-mediated inhibition of CD39 resulted in compromised BMMSCs differentiation potential.

Conclusion: Here, we clearly demonstrated that chemical inhibition as well as blockade of CD39 using a monoclonal antibody impairs the immunosuppression mediated by MSCs. Moreover, our results suggested for the first time that CD39 has an important function in colony formation ability as well as in differentiation potential of MSCs. These novel findings improve our understanding of the mechanistic role of CD39 in MSC function and thus can contribute to the amelioration of MSC-based therapies for the treatment of autoimmune diseases as well as their use in regenerative medicine.
SZT-V03

\section{Exosomes differentially modulate the osteogenic and adipogenic induction of bone marrow-derived mesenchymal stem and progenitor cells}

T. Lener ${ }^{1},{ }^{\star} M$. Gimona², D. Streif², D. Peckl-Schmid², M. Öller ${ }^{1}$, S. Laner-Plamberger ${ }^{1}$, K. Schallmoser ${ }^{1}$, E. Rohde Re, $^{2,1}$

${ }^{1}$ SALK, Transfusionsmedizin, Salzburg, Austria

${ }^{2}$ Paracelsus Medical University, SCI-TReCS, Salzburg, Austria

Background: Bone marrow-derived mesenchymal stem/progenitor cells (MSPCs) and endothelial colony forming cells (ECFCs) are attractive candidates for cell therapy. While transplanted MSCs can undergo osteogenic differentiation in a bone environment, MSPCs co-transplanted with ECFCs support vessel formation. Here we tested whether exosomes derived from either progenitor cell type are sufficient to modulate adipogenic and osteogenic lineage induction in MSPCs.

Methods: MSPCs were cultured in 10\% human platelet lysate (HPL) in $\alpha$-MEM. Proliferation, osteogenic and adipogenic induction of MSPCs were tested in vitro in the presence or absence of exosomes derived from either MSPCs (homotypic exosomes) or ECFCs (heterotypic exosomes). Manual cell counting, colony formation and continuous impedance assays (xCELLigence ${ }^{\circledR}$ from ACEA) were employed. Flow cytometry analysis was used to monitor surface marker presentation. Fluorescence and phase contrast microscopy was performed to visualize exosome uptake, and osteogenic and adipogenic differentiation, respectively.

Results: We show that homotypic but not heterotypic exosomes potentiate osteogenic and adipogenic induction in MSPCs. After osteogenic induction homotypic exosome stimulation maintains the secretion and deposition of mineralizing matrix in MSPCs in the absence of inductive supplement. In contrast, heterotypic exosomes reduce the osteo- and adipogenic differentiation in MSPCs, but stimulate their proliferative potential. The levels of MSPC markers CD73, 105 and 90 remain constant during the course of 14 days of osteogenic and adipogenic differentiation. However, surface presentation of CD9 (Tetraspanin 29) is decreased in cells induced for adipogenic differentiation, but moderately increased in osteogenically induced cells. This variation can be faithfully identified as early as day 6/7 post induction, prior to any detectable Alizarin Red or Sudan 3 positive staining. Conclusion: Homotypic exosomes promote osteogenic and adipogenic induction of MSPCs, while heterotypic exosomes stimulate proliferation. To our knowledge this is the first description of an enhanced lineage induction of MSPCs by homotypic exosomes. In vivo experiments are, however, required to validate these in vitro effects for a potential future use of stem cell exosomes in regenerative therapeutic protocols

\section{SZT-V04 \\ Human mesenchymal stem cells promote Interferon-a production in human plasmacytoid dendritic cells in a Toll-like receptor 9-dependent manner}

\author{
${ }^{\star} P$. Ensel ${ }^{1}$, P. Nold ${ }^{1,2}$, G. Michel', G. Bein ${ }^{1}$, A. Neubauer, \\ C. Brendel ${ }^{1}$, H. Hackstein ${ }^{1,2}$ \\ ${ }^{1}$ University of Gießen, Clinical Immunology and Transfusion Medicine, \\ Gießen, Germany \\ ${ }^{2}$ University of Marburg, Marburg, Germany
}

Background: Human mesenchymal stem cells (hMSC) represent important multipotent cells hitherto believed to exert primarily immunosuppressive effects on human dendritic cells. We show here that this view is incomplete by providing evidence that hMSC can efficiently promote bioactive Interferon-alpha (IFN- $\alpha$ ) production of purified plasmacytoid dendritic cells (pDC). pDC express high levels of Toll-like receptor 7 (TLR7) and TLR9 and are the major IFN- $\alpha$ producers in the immune system. IFN- $\alpha$ represents a key antiviral and antineoplastic cytokine that is frequently used in clinical therapy.

Methods: hMSC were generated from bone marrow employing standard procedures and human BDCA $-4^{+}$pDC precursors were microbead-sorted from human buffy coat samples. Cocultures of purified pDC and hMSCs 
were stimulated through different toll-like receptor (TLR) ligands and pDC activation was analyzed by flow cytometry and ELISA.

Results: Triggering of hMSC pDC cocultures through TLR7 (R848) did not significantly affect IFN- $\alpha$ production of purified pDCs $(n=9)$. In contrast TLR9-dependent activation of hMSC pDC cocultures resulted in marked upregulation (\&gt; $200 \%$ ) of IFN- $\alpha$ production in comparison to pDC-only or hMSC-only controls $(n=6$; p\&lt;0.0001). Moreover, MSC$\mathrm{pDC}$ transwell coculture experiments revealed that soluble, MSC-derived factors are responsible for TLR9-dependent triggering of IFN- $\alpha$ production in $\mathrm{pDC}(\mathrm{n}=6 ; \mathrm{p}=0.0005)$.

Conclusion: These data indicate that hMSC are not per se immunosuppressive. The results identify TLR9 as a new candidate pathway circumventing the immunosuppressing function of hMSC. Given the fact that IFN- $\alpha$ plays a critical role in antiviral immunity, our results reveal antiviral and antineoplastic capacity of hMSC through pDC activation.

\section{SZT-V05}

\section{Screening the entire circulating blood volume for CTCs:} Diagnostic Leukapheresis increases significantly CTC detection rates in patients with non-metastatic cancer

*J.C. Fischer ${ }^{1}$, D. Niederacher ${ }^{2}$, E. Honisch ${ }^{3}$, S. Schuhmacher ${ }^{3}$, N. Schmitz', S. Mohrmann', U. Nitz', J. M. Rox' , F. Wenzel', W.T. Knoefel ${ }^{3}$, N.H. Stoecklein ${ }^{3}$

${ }^{1}$ Medical Faculty, University Hospital of the Heinrich-Heine-University Düsseldorf, Institute for Transplantation Diagnostics and Cellular Therapeutics, Düsseldorf, Germany

${ }^{2}$ Medical Faculty, University Hospital of the Heinrich-Heine-University Düsseldorf, Obstetrics and Gynecology, Düsseldorf, Germany

${ }^{3}$ Medical Faculty, University Hospital of the Heinrich-Heine-University

Düsseldorf, General, Visceral and Pediatric Surgery, Düsseldorf, Germany

${ }^{4}$ Bethesda Hospital, Breast-Center Niederrhein , Mönchengladbach, Germany

Background: Circulating tumour cells (CTCs) hold promise as relevant biomarkers for early detection of systemic cancer spread, surrogate markers for therapy monitoring and for direct access to the molecular characteristics of early and advanced metastatic disease. Major drawbacks in the past twenty years have been the infrequent and unreliable detection of (CTCs) and thus far impeded the clinical use, partly based on the restricted blood volume (1-10 mL) used for testing for CTCs. We therefore set out to assess for the first time the number of CTCs in the entire circulating blood volume. Methods: We screened apheresis products (LA) generated from 2 - 25 L of blood per patient to achieve this. 85 LA products from cancer patients and healthy controls were screened for CTCs. First, we studied a historical cryopreserved sample collective $(n=48)$ from 24 M0 breast cancer patients using immunohistochemistry with an anti-cytokeratin (CK) antibody. Single cell comparative genomic hybridization $(\mathrm{CGH})$ was used to validate the malignant nature of $\mathrm{CK}+$ cells. Then we performed a validation study (collective2) in prospectively harvested diagnostic LA (DLA) samples from breast and pancreatic cancer patients $(n=24)$. We used CellSearch ${ }^{\circledR}$ to directly compare the CTC prevalence in 1-3 ml of LA samples with $7.5 \mathrm{~mL}$ peripheral blood $(\mathrm{PB})$. Results were validated using sex matched healthy volunteer LAs as well as in vitro spiking experiments.

Results: In almost all of the breast cancer patients (cohort 1, 23/24) we found $\mathrm{CK}+$ cells within the LA product. From 19 patients single CK+ cells were successfully isolated and analyzed. 16 of those 19 patients showed an imbalanced CGH profile typical for breast cancer in these $\mathrm{CK}+$ cells. Comparing prospectively autologous $7.5 \mathrm{~mL}$ blood samples with LA-products revealed a significantly increased CTC detection prevalence: $88 \%$ in LA samples compared to $41 \%$ in $7.5 \mathrm{~mL} \mathrm{~PB} \mathrm{(} \mathrm{p}=0.004)$, using the CellSearch ${ }^{\circledR}$ device. Strikingly, median CTC counts in 1-3 ml DLA-product were markedly higher than in $7.5 \mathrm{~mL}$ PB (10.0 vs. 0.0; $\mathrm{p}=0.002$ ). The calculated median CTC number for whole DLA samples was 709 (range: 0-7728).

Conclusion: Using this approach, CTCs can be detected in more than $90 \%$ of non-metastatic patients with breast and pancreatic cancer, which was previously impossible. We further show that diagnostic leukapher- esis can be easily combined with the FDA-approved CellSearch system for standardized enumeration of CTCs. Finally, molecular assays can be applied to leukapheresis-derived CTCs enabling direct and early characterization of therapy-escaping variant CTCs, which may prove useful in therapy trials and future patient management.

\section{SZT-V06 \\ Revision of the human hematopoietic tree: Granulocyte subtypes derive from distinct hematopoietic lineages}

\section{A. Görgens' ${ }^{1}$ S. Radtke ${ }^{1}$, M. Möllmann ${ }^{2}$, M. Cross ${ }^{3}$, J. Dürig ${ }^{2}$,} P.A. Horn ${ }^{1},{ }^{*}$ B. Giebel ${ }^{1}$

${ }^{1}$ Universitätsklinikum Essen, Institut für Transfusionsmedizin, Essen, Germany

${ }^{2}$ Universitätsklinikum Essen, Klinik für Hämatologie, Essen, Germany ${ }^{3}$ Universität Leipzig, Abteilung für Hämatologie, internistische Onkologie und Hämostasiologie, Leipzig, Germany

Background: The classical model of human hematopoiesis predicts a dichotomous lineage restriction of multipotent hematopoietic progenitors (MPP) into common lymphoid and common myeloid progenitors (CLPs and CMPs respectively). However, this idea has been challenged by the identification of lymphoid progenitors retaining partial myeloid potential (e.g. LMPPs), implying that granulocytes can arise within both the classical lymphoid and myeloid branches.

Methods: Here, we resolve this issue by studying the lineage potentials of cell populations separated by cell surface CD133 expression, i.e. CD34+CD133+ (CD133+) and CD34+CD133low (CD133low) cells, in vitro and in vivo.

Results: We show that: i) basophils and eosinophils derive from a common progenitor (EoBP); ii) EoBPs derive in turn from CD34+CD133low erythro-myeloid progenitors that have no neutrophil potential, but do give rise to erythrocytes and megakaryocytes; iii) these erythro-myeloid progenitors (EMPs) are presumably produced together with LMPPs which hold lymphoid, neutrophil and macrophage but no eosinophil, basophil, megakaryocytic or erythroid potential from common CD34+CD133+ MPPs and iv) at least two different qualities of CD133+CD34+ cells can engraft into NSG mice as multipotent progenitors either retaining or lacking erythro-myeloid potential.

Conclusion: Our findings challenge the concept of a common myeloid progenitor and let us propose a new model of early human hematopoiesis. This model restores the dichotomic character of the classical hematopoietic model and includes the existence of lymphoid precursors with partial myeloid potentials

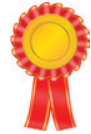

\section{SZT-V07}

\section{Zinc-finger nuclease-mediated correction of} RS-SCID

*S. Rahman ${ }^{1,2}$, J. Kühle', C. Reimann', M. Maeder ${ }^{3}$, H. Riedel', T. Cantz ${ }^{5}$, J.K. Joung ${ }^{3}$, A. Schambach ${ }^{2}$, T. Cathomen ${ }^{1,2,6}$

${ }^{1}$ University Medical Center Freiburg, Center for Chronic Immunodeficiency, Laboratory of Cell and Gene Therapy, Freiburg, Germany

${ }^{2}$ Hannover Medical School, Institute of Experimental Hematology, Hannover, Germany

${ }^{3}$ Massachusetts General Hospital, Molecular Pathology Unit, Charlestown, United States

${ }^{4}$ Robert C. Byrd Health Science Center, Department of Biochemistry, Morgantown, United States

${ }^{5}$ Hannover Medical School, JRG Stem Cell Biology, Hannover, Germany ${ }^{6}$ University Medical Center Freiburg, Department of Transfusion Medicine, Freiburg, Germany

Background: Severe combined immunodeficiencies (SCID) are the most severe form of inherited blood disorders. Failure of the adaptive immune response is due to the absence of $\mathrm{T}$ and/or B/NK cells. The NOD.SCID mouse is a valuable model for human SCID in general and for radio- 
sensitive SCID (RS-SCID) in particular. The T-/B- immune phenotype is caused by a point mutation in exon 85 of the $p r k d c$ gene, which encodes for the DNA repair factor DNA-PKcs.

Methods: To correct the RS-SCID underlying mutation, we generated zinc-finger nucleases (ZFNs) that target intron 84 of the prkdc locus with the aim to restore DNA-PKcs function by integrating a donor DNA encompassing a splice acceptor followed by exons $85 / 86$ and a selection cassette. Results: NOD.SCID-derived ear fibroblasts that underwent gene targeting at the prkdc locus revealed restored DNA-PKcs dependent signaling and reduced sensitivity to the radiomimetic drug bleomycin. Next, we generated NOD.SCID-derived induced pluripotent stem cells (iPSCs) Selected clones contained an intact karyotype and gave rise to all three germ layers in teratoma assays. Upon ZFN-based correction, $\sim 90 \%$ of iPSC clones showed targeted integration of exon 85/86 into intron 84 . As expected, both corrected and uncorrected iPSC clones could be differentiated to DN2-stage $\mathrm{T}$ cell precursors $\left(\mathrm{CD} 44^{+} / \mathrm{CD} 25^{+}\right)$in vitro, but only corrected cells with restored $p r k d c$ expression gave rise to double-positive $\mathrm{CD}^{+} / \mathrm{CD}^{+} \mathrm{T}$ cells, suggesting that DNA-PKcs dependent $\mathrm{T}$ cell receptor recombination was reestablished.

Conclusion: In conclusion, our findings demonstrate that ZFN-based genome engineering can be applied to correct the RS-SCID phenotype in iPSCs by restoring DNA-PKcs dependent signaling and hence may present a paradigm for the generation of patient-derived cell therapeutics.

\section{SZT-V08 \\ HOXB4 cooperates with Runx1 to promote the development of hematopoietic cells at the hemogenic endothelium stage, in vitro}

${ }^{*}$ N. Teichweyde, C. Meyer, S. Skibbe, P.A. Horn, H. Klump

University Hospital Essen, Institute for Transfusion Medicine, Essen, Germany

Background: Hematopoietic differentiation of pluripotent stem cells and expansion of emerging stem and progenitor cells (HSPCs), in vitro, are strongly enhanced by ectopic expression of the homeodomain transcription factor HOXB4. However, the underlying mechanisms are still ill-defined Therefore, we investigated its influence on major fate decisions during different stages of embryonic stem (ES-) cell differentiation, in vitro.

Methods: To follow mesodermal cell fate decisions during ESC differentiation we retrovirally expressed HOXB4 or a 4-Hydroxytamoxifen (4-OHT)-inducible HOXB4-ERT2 fusion protein in different ESC-lines allowing to detect activation of endogenous Brachyury (Bry::GFP; J. Fehling, Ulm) or Runx1 expression (Runx1::Venus; S.-I. Nishikawa, Kobe) or in Runx ${ }^{(--)}$ESCs containing a Doxycyclin inducible Runxl CDS (iRunx; G. Lacaud, Manchester). These lines were differentiated as Embryoid Bodies (EBs) for 6 days and, after dissociation, cultured on OP9 stroma cells for different periods of time. Differentiation cultures were periodically evaluated cyto-morphologically and by immunodetection of lineage-associated surface antigens by fluorescence microscopy and flow cytometry.

Results: HOXB4 mediated an increase in the number of circular, sheetlike structures which expressed EPCR (CD201), VE-Cadherin (CD144), PECAM1 (CD31) and were capable of Dil-ac-LDL uptake. When differentiating Runx1: Venus reporter ESCs expressing HOXB4, the emerging Runx1/Venus ${ }^{+} \mathrm{CD}_{4} 1^{+}$hematopoietic cells were associated with such sheet-structures, presumably representing hemogenic endothelium layers. HOXB4 did not induce or alter initial Runxl expression during EB-development. However, during co-cultivation it promoted the appearance of a subpopulation expressing increased levels of Runx1, which contained all arising CD45 ${ }^{+}$cells. 4-OHT induction of HOXB4 in iRunx 1 cells uncovered that both transcription factors cooperate in hemogenic endothelium to promote its development to $\mathrm{CD} 41^{+} / \mathrm{CD} 45^{+}$stem and progenitor cells.

Conclusion: Whilst HOXB4 alone strongly promoted the formation of hemogenic endothelium structures, CD45 ${ }^{+}$cells were only generated after additional induction of Runx1 expression. So far, our results suggest that the transcription factors HOXB4 and Runx1 cooperate and may even interdepend during the transition of the hemogenic endothelium to definitive hematopoietic stem and progenitor cells, at least during ESC differentiation, in vitro.

\section{Poster: Stammzelltransplantation \&} Stammzellbiologie

\section{SZT-P01 \\ Severe combined deficiency of innate and acquired immunity caused by an IKBKB mutation}

\author{
*U. Pannicke' ${ }^{1}$, B. Baumann², S. Fuchs ${ }^{3}$, P. Henneke ${ }^{3}$, S. Borte $^{4}$, \\ A. Rensing-Eh/ ${ }^{3}$, H. Schrezenmeier ${ }^{1,5}$, T. Wirth ${ }^{2}$, S. Eh/ ${ }^{3}$, M.L. \\ Schroeder ${ }^{6}, K$. Schwarz ${ }^{1,3,5}$ \\ ${ }^{1}$ Institut für Transfusionsmedizin, Universität, Ulm, Germany \\ 2Institut für Physiologische Chemie, Universität, Ulm, Germany \\ ${ }^{3}$ Centrum für Chronische Immundefizienzen, Universität, Freiburg, Germany \\ ${ }^{4}$ Division of Clinical Immunology and Transfusion Medicine, Karolinska \\ Institute, Stockholm, Schweden, Germany \\ ${ }^{5}$ Institut für Klinische Transfusionsmedizin und Immungenetik, DRK- \\ Blutspendedienst BW-Hessen, Ulm, Germany \\ ${ }^{6}$ Department of Pediatrics and Child Health, University of Manitoba, \\ Winnipeg, Kanada, Germany
}

Background: We analyzed the molecular basis of severe combined immunodeficiency (SCID) in four patients from four independent families of a small population of homogenous ethnicity. The patients presented within the first months of life with failure to thrive, oral thrush and invasive bacterial and viral infections.

All patients showed normal numbers of B and T cells and suffered from agammaglobulinemia. Three patients had markedly reduced numbers of NK cells. B and T cells were of naïve phenotype. T cells showed reduced responses to anti-CD3/CD28 treatment. Reactivity of NK cells against K562 target cells was extenuated.

Methods: Assuming an autosomal recessively inherited monogenetic defect, we performed homozygosity mapping.

Results: We identified a region of interest around the centromere of chromosome 8 . We detected a homozygous insertion of one nucleotide in the open reading frame of $I K B K B$ in all patients predicting a frameshift with premature termination of the protein.

Western Blot analysis for the encoded IKK $\beta$ protein did not show any residual protein in fibroblasts and PBMC suggesting a null mutation. IKK $\alpha$ and NEMO expression was reduced.

Functional testing of NF- $\kappa \mathrm{B}$ pathways was performed in fibroblasts. Phosphorylation of IkBa following stimulation with $\mathrm{TNF} \alpha$ was considerably decreased. The binding of $\mathrm{NF}-\kappa \mathrm{B}$ to DNA after stimulation with TNF $\alpha$ or PMA was markedly reduced. Moreover, TLR4 and TLR5 triggered expression of IL- 6 was decreased whereas IL-6 production following stimulation with TNF $\alpha$ or IL- $1 \beta$ was normal.

Conclusion: These data indicate that IKK $\beta$ is not necessary for human $B$ and $\mathrm{T}$ cell development but it is indispensable for lymphocyte activation.

\section{SZT-P02 \\ Crucial Role of Natural Killer Cells in Pediatric Haploidentical Stem Cell Transplantation}

\section{${ }^{*}$ B. Wagner' ${ }^{1}$ A. Scharpf', N. Specht ${ }^{1}$, B. Lowigus ${ }^{1}$, R. Henschler ${ }^{1}$, M.H. Albert ${ }^{2}$}

${ }^{1} \mathrm{KUM}$, Transfusionsmedizin, Muenchen, Germany

${ }^{2}$ KUM, Dr. von Haunersches Kinderspital, Muenchen, Germany

Background: Allogeneic hematopoietic cell transplantation (HCT) has been gaining importance as a curative mode of treatment in various malignant and non-malignant diseases. Progress in methods of $\mathrm{T}$ cell depletion enabled the use of haploidentical (HI) donors as an option: in contrast to CD34+ve enrichment (CD34+E) CD3/19 depletion (CD3/19D) preserves NK cells and monocytic/myeloid cells, e.g. accessory cells or CD34-ve HPCs. We report on children lacking a MUD thus undergoing HIHCT with a CD3/19 depleted and a CD34+ enriched graft, respectively.

Methods: 11 children aged 1-20 with AML/ALL/MDS (5), neuroblastoma (3), hepatoblastoma (1) and inherited immunodeficiency (2) were 
transplanted with CD3/19 depleted and CD34+ enriched grafts each according to disease specific protocols. From $11 \mathrm{H}$ parent donors (9 f, $2 \mathrm{~m}$ ) 13 HSC-As were collected and subjected to 11 CD3/19 depletion and 11 $\mathrm{CD} 34+$ enrichment procedures. HSC-As, intermediate and final products were analysed on a blood counter and a flow cytometer measuring HCT, PLT, WBC, CD34+ HPCs, CD14+ monocytes and lymphocyte subsets including viability.

Results: HSC-As contained in the mean $0.70 \%+0.55 \mathrm{CD} 34+\mathrm{HPCs}$ corresponding to $31.8+23.1 \times 10 \mathrm{e} 6 \mathrm{CD} 34+/ \mathrm{kg} \mathrm{BW}$ and $29.5+26.6 \times 10 \mathrm{e} 5 \mathrm{NK}$ cells $/ \mathrm{kg} \mathrm{BW}$ of the recipient. After CD3/19D and CD34+E T or B cell depletion averaged $3.2 \operatorname{logs}+0.6$ vs. $5.0 \operatorname{logs}+0.5$ and $3.3 \operatorname{logs}+0.7$ vs. 3.2 logs +0.5 . After either CD3/19D or CD34+ E, $81.6 \%+10.9$ vs. $79.7 \%+11.9$ of HPCs and $56.1 \%+21.9$ vs. $0 \%+0$ of NK cells were eluted, respectively. The following mean cell doses per kg BW were infused: CD34+ HPCs $18.6 \times 10 \mathrm{e} 6+6.4$, T cells $1.0 \times 10 \mathrm{e} 5+0.1$, B cells $1.7 \times 10 \mathrm{e} 5+1.9$ and $\mathrm{NK}$ cells $184.0 \times 10 \mathrm{e} 5+235.8 .8$ patients achieved primary engraftment with a median of 14 and 13 days to $>1 \mathrm{WBC} / \mathrm{nl}$ and $>50 \mathrm{PLT} / \mathrm{nl}$, respectively. Two of the three others had received the lowest NK cell doses 5.5 and 5.8 $\mathrm{x} 10 \mathrm{e} 5 / \mathrm{kg}$. This was not only related to the immunomagnetic separation procedure, but also to the low NK count in the HSC-As from their donors. In only 2 children mild acute GVHD (Grade I and Grade II) occurred.

Conclusion: Our results agree with those from Handgretinger and co-workers, who discovered the crucial role of NK cells in the application of T/B lymphocyte reduced grafts in pediatric HIHCT. Hence, further studies increasing NK cell availability for transplantation are required.

\section{SZT-P03 \\ Diagnostic vaccination of pbsc-donors as a tool for differentiation of weak-positive HBV infectious parameters prior to transplantation}

M. Meyer ${ }^{1}$, K. Dahse ${ }^{1}$, I. Trestyanszki ${ }^{1}$, B. Zimmermann ${ }^{1}$, S. Kaun ${ }^{2}$, H. Thomssen ${ }^{2}$, B. Hertenstein ${ }^{2}$, M. Sandkamp ${ }^{3}$, W. Gebauer $^{4}$, F.F. Wagner ${ }^{5}$, C.G. Schüttler ${ }^{6}{ }^{\star}$ E.K. Petershofen ${ }^{4}$ ${ }^{1}$ DRK Blutspendedienst NSTOB, Institut Bremen-Oldenburg, Bremen, Germany

${ }^{2}$ Klinikum Bremen-Mitte, Zentrum Innere Medizin, Medizinische Klinik I, Bremen, Germany

${ }^{3}$ LADR GmbH, MVZ Bremen, Bremen, Germany

${ }^{4}$ DRK Blutspendedienst NSTOB, Institut Bremen-Oldenburg, Oldenburg,

Germany

${ }^{5}$ DRK Blutspendedienst NSTOB, Institut Springe, Springe, Germany

${ }^{6}$ Universitätsklinikum Giessen \& Marburg, Institut für Medizinische

Virologie, Nationales Konsiliarlabor HBV\&HDV, Giessen, Germany

Introduction: According to German Pharmaceutical Law (AMG) it is not allowed to use allogeneic infectious products for therapy. In order to overcome an unclear situation prior to matched related donor transplantation (mrd-tx) with a weak-reactive/positive Anti-HBc, a diagnostic vaccination step was used to differentiate between a relevant infection of the pbsc-donor or an unspecific test result.

Case history: A female patient was diagnosed for AML-M4. Her brother was predicted HLA-identical and immediately selected as pbsc-donor for matched related donor transplantation (mrd-tx). Pre-collection samples were analysed for serum parameters by the clinic including infectious parameters, which were negative. In this family case pbsc apheresis and cryoconservation was performed prior to conditioning of the patient. During work up of the pbsc-product a weak positive test result was found for Anti-HBc, which was in turn also repeated by other involved laboratories. The product was therefore determined "potentially infectious" and kept in quarantine.

Methods: To overcome this unclear situation a diagnostic active HBVvaccination was offered to the donor: in this procedure test results are checked 10-14 days after vaccination to investigate immediate fast boostering effects. Fortunately, the donor's test results were negative and the transplantation procedure was carried out. After successful mrd-tx the patient recovered and HBV parameters (serum/ NAT) were negative, even after 12 month.
Conclusions: (A) Diagnostic vaccination of donors is a valuable tool for discrimination if weak positive infectious parameters are clinically relevant or unspecific: fast and high titers of anti-HBs after 10-14 days would reveal a boostering of a previous infection with loss of HBV-specific antibodies whereas a non-response is unlikely for this event. (B) For future procedures it is advisable to let test infectious parameters of pre-collection samples in the lab which is in duty for the apheresis/production unit.

\section{SZT-P04 \\ Relocation of cryopreserved therapeutic and diagnostic samples}

${ }^{*}$ T. Müller ${ }^{1}$, T. Schmidt ${ }^{2}$, U. Schön ${ }^{2}$, V. von Walcke-Wulffen ${ }^{1}$

${ }^{1}$ BioKryo $\mathrm{GmbH}$, Sulzbach, Germany

${ }^{2}$ Fraunhofer IBMT, St. Ingbert, Germany

Background: Here, we evaluate the relocation of samples stored under cryogenic conditions in the gas phase of liquid nitrogen in between two German cities (range: $700 \mathrm{Km}$ ). For this purpose we logged the temperature within the cryostorage vessels and measured vibrations affecting the vessels during the transport.

Our intention was, to establish a secure system of relocation with closed cooling chain and minimized external impacts to prevent any changes in the cryopreserved samples.

Methods: To evaluate the relocation of cryopreserved samples, the BioKryo $\mathrm{GmbH}$ in collaboration with the Fraunhofer IBMT reviewed the whole transport process. For this, cryo vessels were equipped with sensors recording the temperature within the vessels and the vibrations affecting the tanks.

The cryostorage tank Cryotherm Biosafe 420, modified with hover cushion brackets, was equipped with the control devices Biosafe Control- $\beta$ and Adur- $\beta$ to measure and $\log$ the temperature. During the relocation, vibrations were logged by the device National Instruments NI DAQPad-6251 Pinout, which was fixed to the cryo vessels by a force-locked connection. The signals of the sensors were transiently recorded with a sampling rate of $200 \mathrm{kHz}$.

Results: Analysis of the temperature and the vibrations appearing during the transport indicate, that the samples were constantly stored below $-150^{\circ} \mathrm{C}$ throughout the whole relocation process, which is clearly in the range of save cryostorage temperatures, and that there was no significant external impact on the samples during the transport.

Conclusion: Cryogenic conditions exist at temperatures of less than $-130^{\circ} \mathrm{C}$. This, considered as a save cryostorage temperature range, ensures that chemical processes in the samples are reduced to a minimum and morphological changes are prevented.

The system of relocation of whole cryostorage tanks established by the BioKryo GmbH and the Fraunhofer IBMT is an optimal way to guarantee a secure transport of cryopreserved samples including closed cooling chain and minimized external impacts.

\section{SZT-P05 \\ Engineering the human K562 erythroleukemia cell line to serve as supplement for the long term culture of Plasmodium vivax}

\section{Grätz ${ }^{1},{ }^{\star}$ R. Kronstein-Wiedemann ${ }^{1}$, P. Milanov' ${ }^{1}$ E. Pasini ${ }^{2}$ T. Tonn ${ }^{1}$}

${ }^{1}$ DRK-Blutspendedienst Nord-Ost, experimentelle Transfusionsmedizin, Dresden, Germany

${ }^{2}$ Biomedical Primate Research Centre, Rijswijk, Netherlands

Background: Plasmodium vivax (Pv) infections affects $\sim 200$ million people/year, however Malaria vaccines or therapies are not yet available, which in part is hampered by the inability to long-term culture Pv in vitro. So far, only short-term cultures requiring repeated isolation of premature red blood cells from bone marrow or cord blood. Here, establishing a "red 
blood cell matrix" would facilitate Pv culture and ultimately the development of vaccines. Our study aims to establish culture conditions that allow differentiation of K562 cells into enucleated erythroid cells, serving as a constant source for long-term Pv cultures.

Methods: Since K562 cells do not express the Duffy blood group antigen receptor (Fy), essential for $\mathrm{Pv}$ invasion, the Duffy FyB receptor was stable introduced into K562 cells by lentiviral gene transfer. Transduced cells were plated in methylcellulose and individual clones were picked $(n=24)$. Clones with more than $60 \%$ of benzidine positive cells after hemin stimulation were used for further analysis. An array of agents with differentiation potential was screened for their ability to induce erythroid differentiation of K562-FyB transgenic clones. Cells were cultured for 7 and 14 days in presence of increasing concentration of ARA-C or SAHA, known as potential stimulators of erythroid differentiation. Effects were analysed by quantification of total cell numbers, CD45-CD71+ cells and May-Grünwald-Giemsa staining. Hb expression was detected by Benzidin staining, FACS and PCR.

Results: Among the reagents tested, only ARA-C and SAHA yielded erythroid phenotypes. ARA-C, in a concentration of $200 \mathrm{nM}$, reduces proliferation of K562 cells up to $85 \%$ compared to the non-induced control. Furthermore ARA-C induces production of fetal hemoglobin in K562 cells up to $30-40 \%$. Analysis of mRNA accumulation of embryonic, fetal and adult globin chains by PCR showed a concentration-dependent increase in alpha and gamma globin, but lack of beta-globin expression. Expression of both embryonic hemoglobin chains were non-dosis dependent. Flow cytometric analysis of ARA-C induced cells showed an increased expression of CD71 but also of CD45. In contrast SAHA resulted in an increase of absolute CD71 expression (15\%) and a reduction of absolut CD45 expression (15\%). Hemoglobin accumulation was found to increase 10 to $15 \%$. Moreover proliferation was inhibited by $50 \%$.

Conclusion: The data show that ARA-C and SAHA induce upregulation of erythroid differentiation markers. Albeit at a low efficacy, we could observe enucleated red blood cell like cells after incubation of individual Fy-transfected K562 clones with Ara-C or SAHA. Seemingly, differentiation of K562 cells to enucleated red blood cells may be possible. Further optimisation of culture conditions to enhance the yield of RBC blasts from K562 is necessary, as is the confirmation of Pv infection susceptibility of these cells.

\section{SZT-P06 \\ Identification of dosage dependent tumor suppressor genes in hematopoietic stem and progenitor cells}

\section{*A.M. Zickler, C. Wenzek, P.A. Horn, S. Heinrichs}

Universitätsklinikum Essen, Institut für Transfusionsmedizin, Robert-KochHaus III, Essen, Germany

Background: Malignant transformation of cells is caused by alterations in oncogenes and tumor suppressor genes. Classical tumor suppressor genes require a bi-allelic inactivation to entail functional consequences in the cell. Recently, this "two-hit hypothesis" has been replaced by a continuum model of tumor suppression. This novel concept implicates gene dosage dependency and encompasses haploinsufficiency and sub-haploinsufficiency of tumor suppressor gene expression.

Methods: Here we report a novel approach to identify such genes using a series of shRNA vectors to knockdown the expression of candidate genes to various levels. To test whether gene dosage effects of these candidates provide a clonal advantage for hematopoietic stem and progenitor cells (HSPCs), we performed competitive reconstitution experiments in vivo. Results: We first tested alpha-Catenin (CTNNA1), a proposed tumor suppressor gene that is located within a commonly deleted region of chromosome $5 \mathrm{q}$ and suggested to be affected in the transformation of HSPCs leading to myeloid malignancies. In a transduction/transplantation experiment, we found that RNAi-mediated knockdown of Ctnnal in HSPCs using a series of 8 different Ctnnal-targeting vectors led to marked expansion of the transduced cells compared to controls in two out of 8 animals. One animal showed a strong dominance of cells with reduced
Ctnnal expression within the HSC compartment including a decreased contribution to differentiation and a bias towards the myeloid lineage. Conclusion: This phenotype is highly reminiscent of a myeloid malignancy in humans. Further in vivo studies are underway to validate our initial finding suggesting a functional role of Ctnnal gene dosage in tumor suppression in HSPCs. We propose that our approach to model gene dosage insufficiency will identify novel tumor suppressor genes involved in hematopoietic stem cell transformation.

\section{SZT-P07 \\ Establishment of an extended in vitro assay to detect the most primitive human hematopoietic cells at a clonal level}

*S. Radtke, A. Görgens, P.A. Horn, B. Giebel

Universitätsklinikum Essen, Institut für Transfusionsmedizin, Essen, Germany

Background: According to the classical model of human hematopoiesis, hematopoietic stem cells give rise to multipotent hematopoietic progenitor cells (HPCs) and become restricted either to the lymphoid or myeloid lineage. Recent discoveries of lymphoid progenitors which retain partial myeloid but lack erythroid potential have challenged this model. These findings coupled with recent results of our group, suggest a new model for early human hematopoiesis according to which GMPs and neutrophils are derivatives of a lympho-myeloid branch and eosinophil and basophils of a erythro-myeloid branch.

Methods: In this project, we aim to further unravel hierarchical relationships, especially within the lympho-myeloid branch. In this respect we plan to extend the clonal myelo/lymphoid-initiating cell (ML-IC) assay which allows to determine the developmental potential of primitive human hematopoietic cells retrospectively. Culture conditions being able to maintain all lineage potentials within arising progeny fractions (we plan to test for NK cell-, T cell-, macrophage-, granulocyte-, megakaryocyteand erythrocyte-potentials) are highly desired.

Here, we analyzed the hematopoietic support of established murine stromal cell line cells (AFT024, OP9, MS5) as well as of primary human mesenchymal stromal cells and of endothelial cells derived from different human tissues. Initially, we tested for long-term myeloid, long-term lymphoid and erythro-myeloid capabilities of expanded $\mathrm{CD} 133^{+} \mathrm{CD} 34^{+}$cells. Results: In co-cultures with cells of the murine stromal cell lines we observed a 2 to 6 -fold stronger expansion of $\mathrm{CD} 133^{+} \mathrm{CD} 34^{+}$cells than on primary endothelial cells (ECFC, HUVEC, PLVEC) or on MSCs either derived from placentas, umbilical cord veins/arteries and AGMs. In contrast, similar rates of long-term culture initiating cells (LTC-IC) were found in all conditions, exclusively within the $\mathrm{CD} 133^{+} \mathrm{CD} 34^{+}$cell fractions. Strikingly, NK cell potentials were highest in $\mathrm{CD} 133^{+} \mathrm{CD} 34^{+}$ cell fractions expanded on endothelial stromal cells. None of the tested primary human or murine cells lines showed a reliable maintenance of erythro-myeloid potentials within the $\mathrm{CD} 133^{+} \mathrm{CD} 34^{+}$cell fractions. Only CD133-CD34 ${ }^{+}$cells were found to contain erythro-myeloid capabilities, the highest content in cell fractions which were expanded on human placental endothelial and MSC lines.

Conclusion: Next we will analyze whether these stromal cells also permit $\mathrm{B}$ cell, T cell and megakaryocyte development. 


\section{SZT-P08}

\section{TCR $\alpha / \beta^{+} T$ - cell depletion: A new promising GvHD prophylaxis in HLA-haploidentical stem cell transplantation}

\section{${ }^{\star} P$. Schauwecker ${ }^{1}$, A. Schulz², M. Hönig ${ }^{2}$, B. Maccari ${ }^{1}$} H. Schrezenmeier ${ }^{1}$, M. Wiesneth ${ }^{1}$

${ }^{1}$ Institute of Clinical Transfusion Medicine and Immunogenetics UIm, Germany, UIm, Germany

${ }^{2}$ Clinic for Pediatric and Adolescent Medicine, University Hospital of UIm, Germany, Ulm, Germany

Background: Graft versus host disease (GvHD) mediated by donor T-cells is still one of the major problems, especially in the setting of HLA-haploidentical transplantation of hematopoietic stem cells. T-cell depletion of the graft is the most effective GvHD prophylaxis but is associated with a significant delay of posttransplant immunoreconstitution and a higher risk of leukemic relapse. Preliminary data show, that specific depletion of TCR $\alpha / \beta^{+}$T-cells may ensure a rapid immunoreconstitution while the graft versus leukemia reaction is maintained, given the fact that TCR $\gamma / \delta^{+}$T-cells and NK-cells are not removed.

Methods: Two patients aged 6 and 7 years and suffering from immunodeficiency diseases received haploidentical peripheral blood stem cell transplants (PBSCT) from their parents after a conditioning regimen containing Fludarabin, Thiotepa, Melphalan and ATG. TCR $\alpha / \beta^{+}$T-cell and $\mathrm{CD} 19^{+}$(B-cell) depletion of the grafts were performed by immunomagnetic separation using the CliniMACS device (Miltenyi Biotec). The grafts contained 11 and $6 \times 10^{6} \mathrm{CD}^{+} 4^{+} / \mathrm{kgbw}, 5$ and $9 \times 10^{6} \mathrm{TCR} \gamma / \delta^{+} /$ kgbw as well as 4 and $5 \times 10^{4} \mathrm{TCR} \alpha / \beta^{+} \mathrm{T}$-cells/kgbw, respectively. Residual B-cells were $8 \times 10^{4} / \mathrm{kgbw}$ in each graft. Recovery after TCR $\alpha / \beta^{+}$ depletion was $87 \%$ and $83 \%$ for CD34 $4^{+}$and $90 \%$ and $84 \%$ for TCR $\gamma / \delta^{+}$ T-cells, respectively. Depletion efficiency was $>3 \log$ for TCR $\alpha / \beta^{+} \mathrm{T}$-cells and B-cells.

Results: Hematopoietic reconstitution was fast (granulocytes $>500 / \mu 1$ at day +10 and day +14 ; platelets $>20.000 / \mu 1$ at day +9 and day +12 ) and both patients developed a complete and stable engraftment. The immunological reconstitution was slightly delayed $\left(\mathrm{CD}^{+}{ }^{+} \mathrm{T}\right.$-cells $<200 / \mu 1$ for more than 3 months in both patients). Using a prophylaxis with MMF and CSA in one and MMF alone in the other patient acute GvHD $\mathrm{I}^{\circ}$ (isolated skin) was observed in both patients, which responded rapidly by a short treatment with systemic steroids. There were no severe infectious complications posttransplant and both patients are alive and well 3 and 5 months after transplantation, respectively.

Conclusion: TCR $\alpha / \beta^{+}$T-cell depletion of the graft can be performed without a significant loss of CD $34^{+}-$, NK- and TCR $\gamma / \delta^{+}$T-cells to prevent severe GvHD and to improve engraftment. In the meantime a clinical trial started to proof the rapid immunoreconstitution and the anti-leukemic effect after PBSCT with this new approach.

\section{SZT-P09}

Adoptive T-cell immunotherapy from third-party donors: Characterization of donors and set up of a T-cell donor registry

C. Sukdolak', S. Tischer', D. Dieks' ${ }^{1}$, C. Figueiredo', L. Goudeva' ${ }^{1}$, H.-G. Heuft ${ }^{1}$, M. Verboom ${ }^{1}$, S. Immenschuh ${ }^{1}$, S. Borchers' ${ }^{2}$, E. Mischak-Weissinger ${ }^{2}$, R. Blasczyk ${ }^{1}$, B. Maecker-Kolhoff ${ }^{3}$, ${ }^{*} B$. Eiz-Vesper ${ }^{1}$

${ }^{1}$ Medizinische Hochschule Hannover, Institut für Transfusionsmedizin, Hannover, Germany

${ }^{2}$ Medizinische Hochschule Hannover, Abteilung Hämatologie,

Hämostaseologie, Onkologie , Hannover, Germany

${ }^{3}$ Medizinische Hochschule Hannover, Pädiatrische Hämatologie und Onkologie, Hannover, Germany

Background: Human cytomegalovirus (CMV), Epstein-Barr virus (EBV) and adenovirus (ADV) infection and reactivation are frequent and severe complications of hematopoietic stem cell transplantation (HSCT) and solid organ transplantation in immunocompromised recipients. Adoptive immunotherapy with virus-specific cytotoxic $\mathrm{T}$ effector cells (CTLs) can effectively reconstitute antiviral immunity without causing acute toxicity or increasing the risk of GvHD.

Methods: Treatment with allogeneic virus-specific CTLs from healthy seropositive third-party donors is a veritable option, particularly for infections following allogeneic cord-blood stem-cell transplantation (SCT) or SCT from virus-seronegative donors. To gain more insight into virus-specific memory T-cell pools in healthy subjects and to identify the most efficient antigens for adoptive immunotherapy, we assessed the frequencies of CMV, EBV and ADV-specific memory T cells in 204 HLA-typed healthy donors using peptides and peptide pools available in GMP quality. Results: For each virus, we identified at least 124 (61\%) potential CTL donors with highly significant differences in frequencies of $\mathrm{T}$ cells against six viral antigens of $\mathrm{CMV}, \mathrm{EBV}$ and ADV. Antigen-specific $\mathrm{T}$ cells were detected in $100 \%$ of CMV-seropositive donors $(n=124)$, $73 \%$ of EBV-seropositive donors $(n=195)$ and $73 \%$ of ADV-seropositive donors $(n=196)$. Among the tested antigens frequencies for CMV pp65 and EBV BZLF1 peptide pool were highest. Overall frequencies of peptide-specific $\mathrm{T}$ cells detected by $\mathrm{T}$-cell receptor staining were lower than those of the corresponding peptide pools. Short-term in vitro peptide stimulation assays revealed that, in the case of ADV and EBV, a donor response to a certain peptide may not be determined with prior stimulation. Conclusion: A modified granzyme B ELISpot was established and used to detect T-cell specificity and alloreactivity. Moreover, confirmatory testing for CMV serology using western blot technique revealed 19/143 $(13 \%)$ false-positive results, possibly impacting future analysis and selection of potential stem cell and T-cell donors.

Treatment with allogeneic virus-specific CTLs from seropositive third-party donors may be a feasible therapeutic option for infections following cord-blood SCT or HSCT from virus-seronegative donors.

\section{SZT-P10 \\ Changes of immunological properties of placental derived mesenchymal stem cells over time and reprogramming procedures}

\section{${ }^{*}$ O. Pogozhykh, A. Wiedemann, R. Blasczyk, T. Müller}

Medizinische Hochschule Hannover, Institut für Transfusionsmedizin, Hannover, Germany

Background: In regenerative medicine, mesenchymal stem cells (MSCs) derived from the stroma of several tissues are of special interest due to their capacities of immune modulation in acute inflammation. Their influence on regulatory $\mathrm{T}$ cells (Tregs) could be a useful tool in transplantation of stem cell derived cellular therapeutics. While MSCs from bone marrow, adipose tissue and cord blood are well characterized, the immunological potential of MSC populations retrieved from placental tissues (pMSCs) remains controversial.

Methods: We harvested and cultured mesenchymal stem cells from different placental regions of the common marmoset monkey namely umbilical cord, chorion, amnion and placenta. For reprogramming, amnion derived mesenchymal cells were used. In the presence of TAV, SB431542, PD0325901 and ascorbic acid, the cells were reprogrammed via a lentiviral SFFV driven quad-cistronic vector system in mTESR medium and transferred onto mouse embryonic feeder cells. In this study we investigated immune-modulatory molecules of placental derived MSCs in the preclinical non-human primate model of the common marmoset monkey (Callithrix jacchus). In addition, these cells were compared with induced pluripotent stem (iPS) obtained from pMSCs.

Results: MSCs from umbilical cord, chorion, amnion and placenta were harvested and successfully cultured until passage 20. All cell types displayed characteristic fibroblast-like morphology, high proliferative potential, mesenchymal markers like CD90, CD105, CD73 and Snail1 which they kept in culture over 12 passages. Amnion MSCs displayed the best proliferative quality and were further utilized for reprogramming experiments. Marmoset MSCs and iPS cells expressed several sets of immune-relevant 
molecules like Gal3, PGE2, Sema3A, Sema6D and Sema7A. Whereas no striking changes could be observed in a certain panel of immune-relevant genes for 12 passages, MSC derived iPS cells restored low expression of Gal3 and HGF, but increased expression could be observed for PGE2 and Sema7A. Interestingly, they displayed significant lower levels of HGF and MHC class I compared to bone marrow MSCs.

Conclusion: No changes in gene expression of immune-relevant genes was observed in the placenta-derived MSCs during passaging. Placenta-derived MSCs appear to be unique candidates for reprogramming when clinical application with reduced gene expression of immune-relevant molecules avoiding rejection or inflammatory reactions is desired.

\section{SZT-P11 \\ Human mesenchymal stem cells constitutively express the serine protease granzyme $B$}

\section{${ }^{*} M$. Schuell}

Institut fuer Klinische Transfusionsmedizin und Immungenetik, UIm, Germany

Background: Human mesenchymal stem cells (MSC) are known for their immunoregulatory potential and are allegedly involved in tolerance induction. Several immune cells have been shown to exhibit their regulatory effects via expression of the cytotoxic serine protease granzyme $\mathrm{B}$ including regulatory $\mathrm{T}$ cells and plasmacytoid dendritic cells. Since MSC were recently found to express the GrB inhibitor PI-9, which is primarily expressed by cytotoxic cells, we hypothesized that MSC may also express $\mathrm{GrB}$.

Methods:

Western-Blotting

ELISpot

FACS analyse

Enzymatic analyse

Co-culture of PBMC and MSC

Results: Using various methods including Western immunoblotting, ELISpot, FACS analysis we found that MSC freshly isolated from healthy donor bone marrow biopsies indeed express considerable amounts of $\mathrm{GrB}$ protein. ELISpot assays demonstrated that $\mathrm{GrB}$ was not only expressed but also secreted by MSC. Moreover, a colorimetric GrB activity assay confirmed that $\mathrm{GrB}$ was secreted in its enzymatically active form. Importantly, when testing the effect of MSC on T cells, we found that $\mathrm{CD} 3 / \mathrm{CD} 28$-induced $\mathrm{T}$ cell proliferation can be suppressed by MSC in a GrB-dependent manner.

Conclusion: Future studies have to elucidate which further aspects of the immunoregulatory activity of MSC possibly depend on GrB and which culture conditions may support a suppressive phenotype of these cells including $\mathrm{GrB}$ expression. Our results may open novel approaches to the use of MSC as immunoregulatory cellular therapeutics in inflammatory conditions such as graft-versus-host disease.

\section{SZT-P12}

\section{Stem cell donor search in Turkish families in Germany}

\section{${ }^{*}$ S. Radojska, T. Turul Özgür, B. Gathof}

Uniklinik Köln, Transfusionsmedizin, Köln, Germany

Background: Allogeneic hematopoietic stem cell transplantation (HSCT) still is the only curative therapy for a variety of diseases. The outcome is influenced by degree of HLA matching; an HLA identical sibling is the optimal donor. For up to $30 \%$ of patients in Western Countries an HLA-identical sibling is identified, in Middle Eastern countries for up to $70 \%$ (Rosenmayer A, 2012) due to consanguineous marriages and family size. It might be as high in Turkey due to growing population (Arat $\mathrm{M}, 2008$ ). This study evaluated the results in families from immigrant Turkish patients in Germany.
Methods: A total of 32 patients with haematological malignancies requiring HSCT and their 136 family members examined between January 2007 and April 2013 were retrospectively evaluated. HLA genotypes and patient data (age, underlying disease and gender) were analysed.

Results: 32 patients and their 93 close and 43 extended family members were typed for HLA compatibility. The mean number of siblings per patient examined was $2.22 .8 \%$ of the siblings were found to be HLA identical, whereas $51.8 \%$ and $25.3 \%$ were HLA haploidentical and not identical, respectively. For 3 families an extended family search was performed revealing a total of 18 additional haploidentical donors within relatives. This composes $41.8 \%$ of the extended relatives.

Conclusion: Factors increasing availability of an HLA identical sibling donor are large family size and consanguineous marriages with more shared haplotypes. In the present study HLA-identical donors were lower than expected. This might be due to a decline in consanguineous marriages and family size as a result of cultural and social interactions. Unlike a previous study (Elbjeirami WM, 2013) where older patient age increased the probability of finding a matched family donor, we found a mean age of 38.5 years. This might be due to decreased access to family members living in either countries.

\section{SZT-P13 \\ Identification of an effective early in vivo signaling signature during neo-vasculogenesis by ex vivo proteomic profiling}

\section{R. Rohban ${ }^{1}$, A. Reinisch ${ }^{1}$, N. Etchart ${ }^{1}$, K. Schallmoser ${ }^{2,3}$,} N.A. Hofmann', ${ }^{*}$ E. Rohde ${ }^{2,3}$, D. Strunk ${ }^{1,2,4}$

${ }^{1}$ Medical University of Graz, Stem Cell Research Unit, Graz, Austria ${ }^{2}$ Paracelsus Medical University of Salzburg, Spinal Cord Injury \& Tissue Regeneration Center Salzburg (SCl-TReCS), Salzburg, Austria

${ }^{3}$ Salzburg University Hospital (SALK), Dept. for Blood Group Serology and Transfusion Medicine, Salzburg, Austria

${ }^{4}$ Paracelsus Medical University, Institute of Experimental and Clinical Cell Therapy, Salzburg, Austria

Background: Therapeutic neo-vasculogenesis in vivo can be achieved by the co-transplantation of human endothelial colony-forming progenitor cells (ECFCs) with mesenchymal stem/progenitor cells (MSPCs). The underlying mechanism is not completely understood thus hampering the development of novel stem cell therapies. We hypothesized that proteomic profiling could be used to retrieve the in vivo signaling signature during the initial phase of human neo-vasculogenesis by explantation of vasculogenic plugs after $24 \mathrm{~h}$.

Methods: ECFCs and MSPCs were therefore either transplanted alone or co-transplanted subcutaneously into immune deficient NSG mice. Early cell signaling, occurring within the first 24 hours in vivo, was analyzed using antibody microarray proteomic profiling after explantation. Vessel formation and persistence were verified in parallel transplants for up to 24 weeks.

Results: Proteomic analysis revealed significant alteration of regulatory components including caspases, calcium/calmodulin-dependent protein kinase, DNA protein kinase, human ErbB2 receptor-tyrosine kinase as well as mitogen-activated protein kinases. Caspase-4 was selected from array results as one therapeutic candidate for targeting vascular network formation in vitro as well as modulating therapeutic vasculogenesis in vivo. As a proof-of-principle, caspase-4 and general caspase blocking led to diminished endothelial network formation in vitro and significantly decreased vasculogenesis in vivo.

Conclusion: Proteomic profiling ex vivo thus led us to describe a signaling signature which can be used for target selection to modulate neo-vasculogenesis in vivo. 


\section{SZT-P14}

\section{Reprogramming human somatic cells towards pluripotency and their differentiation to hematopoietic stem and progenitor cells}

\author{
${ }^{*}$ K. Stolp, M. Zuk, P.A. Horn, H. Klump \\ University Hospital, Transfusion Medicine, Essen, Germany
}

Background: At present, somatic gene therapy of hematopoietic stem and progenitor cells (HSPCs) is typically conducted by retroviral vector-based complementation of recessive genetic defects. However, the procedure is associated with a significant risk of therapy-associated leukemogenesis caused by random vector integration into the genome and altered oncogene expression. Induced pluripotent stem (iPS) cells may represent an attractive alternative to somatic stem cells as they allow for homologous recombination, clonal expansion and differentiation towards HSPCs, in vitro.

Methods: iPSC-generation was achieved after transduction of HUVECs with a lentiviral vector co-expressing the cDNAs for human transcription factors $O C T 4, K L F 4, S O X 2$ and $M Y C$ together with a fluorescent protein. Flanking FRT sites allow for the removal of the vector by Flp-recombinase to preclude reactivation of the reprogramming cassette during differentiation. To establish protein-transduction based vector excision, a novel huESC reporter cell line was generated and evaluated using virus particle-mediated Flp-protein transfer.

Hematopoietic differentiation of pluripotent stem cells was achieved using either a two-step protocol with an Embryoid Body (EB) differentiation phase for 8 days followed by co-culture on OP9 stromal cells. Alternatively, cells were differentiated directly on OP9 stroma.

Results: Formation of colonies with embryonic-stem-cell like morphology was observed approx. 15 days after gene transfer with an efficiency of about $1 \%$. They showed hallmarks of pluripotency such as alkaline phosphatase activity, surface expression of SSEA-4, TRA-1-60 and TRA-1-81 as well as re-expression of the endogenous transcription factors $N A N O G$, SOX2, OCT4, LIN28A and DNMT3B. Global transcriptome analysis by RNA-seq revealed significant similarity of two iPS clones to an human ESC-line (H1). As differentiation of human pluripotent stem cells towards HSPCs capable of long-term repopulation in immunodeficient recipient mice has not been established yet, different conditions were tested utilizing the huESC lines $\mathrm{H} 1$ and $\mathrm{H} 9$. To this end, direct differentiation on OP9 stroma appeared to be superior for the generation of $\mathrm{CD} 34^{+}$hematopoietic progenitors.

Conclusion: Human iPSCs were successfully generated from HUVECs using a lentiviral OKSM-vector. As transplantable HSCs have not yet been generated from human pluripotent stem cells, we evaluated different conditions for hematopoietic differentiation, in vitro. Although CD34 cells were generated using a one-step co-culture protocol on OP9 stroma, the overall efficiency needs to be substantially improved. Presumably, insights gained from the HSC-promoting activities of HOXB4 during in vitro HSC development of mouse ESCs will help improve the formation of HSPCs from huESCs and iPSCs.

\section{SZT-P15}

\section{'Rainbow reprogramming' of nonhuman primate common marmoset cells towards pluripotency}

\section{${ }^{*}$ M. Zuk, K. Stolp, S. Skibbe, P.A. Horn, H. Klump}

University Hospital Essen, Institute for Transfusion Medicine, Essen, Germany

Background: Pluripotent stem cells are particularly attractive for transfusion medicine because they can be differentiated towards any somatic cell type including hematopoietic stem cells for transplantation and mature effector cells for transfusion. To test the medical applicability of this notion in a relevant animal model, we are establishing protocols to reprogram cells of the nonhuman primate common marmoset (Callithrix jacchus) towards induced pluripotent stem cells (iPSCs).
Methods: To generate iPSCs, a lentiviral vector co-expressing the cDNAs for the human transcription factors (TFs) OCT4, KLF4, SOX2 and MYC (in the order $5^{\prime}$-OKSM-3') was employed which we routinely use for reprogramming human cells (umbilical vein endothelial cells and dermal fibroblasts) and mouse fibroblasts (tailtip and embryonic fibroblasts). To test the influence of TF stoichiometry on reprogramming efficiency, we independently transduced each factor together with a different fluorescence protein by separate lentiviral vectors. This "rainbow reprogramming" system allows for an estimation of the stoichiometric ratios of all four transcription factors at different time points during iPSC generation by determination of fluorescence intensities and subsequent additive color mixture.

Results: The obtained colonies generated with the "all-in-one vector", expressed alkaline phosphatase (AP), stained positive for SSEA-3 and SSEA-4 but were negative for TRA-1-81 and TRA-1-60 expression. Furthermore, global transcriptome measurements (RNA-seq) revealed that the generated pre-iPSCs somewhat differed from marmoset embryonic stem cells (ESCs) but were much more similar to ESCs than to fibroblasts indicating a partially reprogrammed state. Uncoupled, "rainbow reprogramming" also only led to partially reprogrammed cells which were AP and SSEA-4 positive and partially positive for SSEA-3, TRA-1-81 and TRA-1-60 expression. However, addition of the DNMT inhibitor 5-Azacytidine during reprogramming mediated a heterogenous pattern of colors, partial silencing of the vectors and a higher proportion of pluripotency-associated surface marker positive cells.

Conclusion: The ratios of ectopically expressed reprogramming factors is known to be crucial for iPSC generation. Because of a dedifferentiation block observed with the "all-in-one" OKSM vector in marmoset cells, we used the "rainbow system" to test and simultaneously monitor different TF-ratios during reprogramming in combination with the DNMT inhibitor 5-Azacytidine. To this end, our results suggest that stoichiometries different from those sufficient for human or mouse iPSC generation, in combination with epigenetic modifiers, may promote iPSC generation towards full pluripotency.

\section{SZT-P16 \\ Immune Modulatory Capabilities of Mesenchymal Stem Cells (MSCs)}

\section{${ }^{*}$ M.B. Fischer', B. Rueger 1 , G. Yanagida', E. Steinhuber ${ }^{2}$, H. Stockinger ${ }^{2}, A$. Giurea ${ }^{2,3}$}

${ }^{1}$ Klinik für Blutgruppenserologie und Transfusionsmedizin, Abteilung für Transfusionsmedizin, Wien, Austria

${ }^{2}$ Institut für Immunologie, Wien, Austria

${ }^{3}$ Orthopädie, Wien, Austria

Background: MSC infusion improves the outcome of severe graft-versus-host disease (GVHD), which is a frequent and threatening complication of allogeneic stem cell transplantation. MSCs are regarded as hypo-immunogenic and have been successfully transplanted across human leukocyte antigen barriers without rejection.

Methods: We compared regulatory T (Treg) cells with MSCs in an immunosuppression assay to suppress an OKT3 mediated immune response in purified CD4-positive T cells. Furthermore, we tried to quantitate the expression of forkhead box P3 (FoxP3), a transcription factor associates with histone acetyltransferase and class II histone deacetylases in MSCs isolated from different tissue sources with Treg cells by FACS and western blotting. Results: MSCs from bone marrow or bone, but not from adipose tissue harbored MSCs that showed immune suppressive capabilities in an immunosuppression assay where purified CD4-positive T cells were activated with OKT3 in the presence of antigen presenting cells. While 50000 Treg cells were needed to suppress the OKT3 stimulation in CD4-positive T cells, 5000 MSCs had the same effect. Interestingly, FoxP3 expression in MSCs of different tissue sources varies extensively while FoxP3 expression in CD4-positive was between $6-12 \%$ as determined by FACS. While no FoxP3 expression could be observed in MSCs from adipose tissue, $0,1-3 \%$ of bone marrow and bone MSCs expressed FoxP3 with great variants between donors. These results could be confirmed by western blotting analysis using a different FoxP3 mAb clone. 
Conclusion: In conclusion we can say that MSCs from bone marrow and spongy bone tissue have immune modulatory capabilities and express the transcription factor FoxP3, although with variable intensity.

\section{SZT-P17}

Self-Organizing Capacity of Bone Marrow Stem Cells and Peripheral Tissue Precursor Cells in Forming Capillary Sprouts within Three Dimensional (3D) Fibrin Matrices

\section{${ }^{*}$ M. B. Fischer ${ }^{1}$, B. Rüger ${ }^{2,1}$, G. Yanagida ${ }^{2,1}$}

${ }^{1}$ Orthopädie, Wien, Austria

${ }^{2}$ Klinik für Blutgruppenserologie und Transfusionsmedizin, Abteilung für Transfusionsmedizin, Wien, Austria

Background: New vessels can be formed by sprouting and branching of new capillaries from the preexisting vascular network and subsequent stabilization of these newly formed constructs by abluminal coverage of smooth muscle cells. Alternatively, new vessels can be formed de novo due to vasculogenesis involving endothelial precursor cells and pericytes. Methods: Bone marrow mononuclear cells (BMMNCs, in a cell density of $4,5 \times 10^{5} /$ well were seeded to ensure suspension of single cells distributed in different planes) as well as multiple small fragments of synovial tissue and the wall of the aorta (in a size of $1 / 2 \mathrm{~mm}^{2}$ ) were embedded into three-dimensional (3D) fibrin matrices and cultivated in 24 well plates in M199 medium for 14 days without the addition of growth factors.

Results: Bone marrow derived stem cells showed de novo formation of capillaries after 7-14 days of cultivation in the fibrin gel. The embed ded BMMNCs formed cell cluster expressing endothelial progenitor cell markers CD133, CD34, VEGFR-2 and c-kit, with stellar shaped spreading of peripheral elongated cells forming tube-like structures over time. The developing vascular structures expressed the endothelial markers CD31 and vWF and showed basement-membrane-lined lumina of collagen type IV surrounded by a-SMA expressing mural cells. A cluster frequency of approximately one cluster $/ 10^{6}$ embedded BMMNCs could be observed. When synovial tissue or aorta was embedded into the 3D fibrin matrices sprouts originated from preexisting vessels and from densely packed cell clusters staining positive for precursor cell markers such as CD133. After one week of cultivation sprouts emerging from the embedded synovial tissue or aorta converted to vessels stabilized with smooth muscle actin (SMA) positive pericyte. Lumina occasionally contained nucleated round cells expressing CD45 or erythrocytes. The appearance of vascular structures displaying this degree of complexity was a rare event consistent with the low frequency of cluster forming precursor cells in the tissues and quantification was complicated due to their 3D growth.

Conclusion: In conclusion we can say that bone marrow as well as peripheral tissues like synovial tissue and aorta have a high potential to form new vessels in a primary avascular 3D fibrin gel.

\section{Oral Abstract Session: Therapeutische Hämapherese}

\section{TTH-V01 \\ Extracorporeal photopheresis triggers IL-1 $1 \beta$ production via caspase-1 dependent and independent pathways}

\section{${ }^{\star}$ E. Yakut ${ }^{1}$, C. Jakobs ${ }^{2}$, G. Bein ${ }^{1}$, V. Hornung ${ }^{2}$, H. Hackstein ${ }^{1}$}

${ }^{1}$ University of Gießen, Clinical Immunology and Transfusion Medicine, Gießen, Germany

2University of Bonn, Clinical Chemistry and Pharmacology, Bonn, Germany

Background: Extracorporeal photopheresis (ECP) is a widely used cellbased therapy exhibiting clinical efficacy in heterogenous immune-mediated diseases such as cutaneous $\mathrm{T}$ cell cancer, graft versus host disease (GVHD) and organ allograft rejection. Despite its documented efficacy in cancer immunotherapy, little is known regarding the induction of soluble immunostimulatory mediators by ECP. Here we show for the first time that ECP promotes marked induction of the prototypic immunostimulatory cytokine IL-1 $\beta$ in murine and human cells via caspase 1 -dependent and independent pathways.

Methods: IL-1 $\beta$ modulation by ECP was analysed by ELISA and western-blot assays in human and mouse leukocytes systems including inflammasome gene-deficient mouse models (ASC-KO, AIM2-KO, Caspase 1 KO mice), dendritic cell culture systems and human patients.

Results: ECP effectively primed IL- $1 \beta$ production in a psoralen-dependent manner in human and mouse leukocytes. Western blot analyses and mature IL-1 $\beta$ specific ELISA methods revealed mature IL-1 $\beta$ release and caspase-1 upregulation in ECP-treated leukocytes and dendritic cells. Chemical caspase-1 inhibition only partially suppressed ECP-mediated IL-1 $\beta$ induction. Analysis of ASC-KO, AIM2-KO, and Caspase 1 gene-deficient murine dendritic cells revealed inflammasome- and caspase-1-independent induction of mature IL-1 $\beta$ suggesting different pathways of ECP-mediated IL-1 $\beta$ induction. Additionally, analysis of clinical patients confirmed significantly increased mature IL- $1 \beta$ production in stimulated leukapheresis concentrates and peripheral blood samples after ECP in comparison to pre-ECP controls.

Conclusion: To our knowledge this is the first report providing evidence for IL-1 $\beta$ induction by ECP via caspase- 1 dependent and independent pathways in murine and human cells. Mature IL-1 $\beta$ induction by ECP may provide novel insight into the immunostimulatory activity of photopheresis.

\section{TTH-V02}

\section{Extracorporeal photopheresis promotes selective survival of CD14+ monocytes and immunostimulatory IL- $1 \beta$ production}

\section{*A. Peric, G. Michel, A. Nockher, G. Bein, H. Hackstein}

University of Gießen, Clinical Immunology and Transfusion Medicine Gießen, Germany

Background: Extracorporeal photopheresis represents an important cellular therapy that is used successfully for treatment of cutaneous $\mathrm{T}$ cell cancer, organ allograft rejection and graft versus host disease. Many reports have focused on immunoregulatory activity of ECP but little is known regarding immunostimulatory effects of ECP. Here we have revealed a novel immunostimulatory activity of ECP.

Methods: After experimental ECP with human peripheral blood mononuclear leukocytes we have analysed systematically the modulation of T-cell derived cytokines after CD3/CD28 mAb stimulation and antigen-presenting cell-derived cytokine production after LPS/R848 (TLR4/7/8) stimulation. Survival of all major leukocyte subsets including T, B, NK lymphocytes, monocytes and dendritic cells subsets was quantitated by multiparameter flow cytometry.

Results: ECP abrogated efficiently IL-2, IL-5, IL-6, IL-10, IL-12 and IFN- $\gamma$ production $(>90 \%)$ after T-cell or antigen presenting cell stimulation. In contrast, ECP markedly enhanced PBMC-derived immunostimulatory IL- $1 \beta$ production $(>4-6$ fold elevation; $n=8 ; p<0.0001)$ at clinically relevant UVA $\left(2 \mathrm{~J} / \mathrm{cm}^{2}\right)$ and 8 -Methoxypsoralen $(400 \mathrm{ng} / \mathrm{ml})$ conditions. IL- $1 \beta$ induction was not a result of passive release or cell death since irradiation with higher UVA-doses was unable to increase IL$1 \beta$ levels. Analysis of the surviving cell types after ECP by multiparameter flow cytometry revealed that all lymphocyte subsets as well as dendritic cell subsets went into apoptosis after ECP. In contrast, ECP promoted almost unaffected survival of $\mathrm{CD} 14^{+}$monocyte subsets (viability $>80 \%$ ). ECP experiments with highly purified human monocytes confirmed marked IL- $1 \beta$ induction $(n=4, p<0.001)$ indicating that ECP-activated monocytes represented the cellular source of IL-1 $\beta$.

Conclusion: Here we have identified a novel immunostimulatory pathway that is triggered by ECP in viable human monocytes. ECP-mediated induction of the prototypic activatory cytokine IL- $1 \beta$ may provide novel insight into the reported clinical activity of ECP for cancer therapy. 


\section{TTH-V03}

\section{Flow Cytometry to Measure T Cell Death and Reduced Proliferation after Photopheresis}

\section{Grabmer ${ }^{1,2}$, *D. Peckl-Schmid ${ }^{2}$, K. Schallmoser ${ }^{1,2}$, E. Rohde ${ }^{1,2}$ \\ ${ }^{1}$ Salzburg University Hospital (SALK), Blood Group Serology and Transfusion Medicine, Salzburg, Austria \\ ${ }^{2}$ Paracelsus Medical University (PMU), Spinal Cord Injury \& Tissue \\ Regeneration Center Salzburg (SCI-TReCS), Salzburg, Austria}

Background: Extracorporeal photopheresis (ECP) is a well-established immunomodulatory therapy. Its clinical benefit has been demonstrated in a wide variety of diseases including cutaneous T-cell lymphoma, acute and chronic graft-versus-host disease, systemic lupus erythematosus and solid organ transplantation. Remarkably, the exact mode of action of ECP remains poorly understood and routine characterization of ex vivo treated lymphocytes is still lacking. In this study we combined a T cell proliferation assay with life-dead cell discrimination to prove efficiency and to validate the ECP procedure.

Methods: Peripheral blood mononuclear cells (PB-MNCs) from buffy coats of healthy volunteer donors $(n=6)$ were diluted and, after addition of 8-methoxypsoralen (8-MOP), UV-A irradiated with an offline ECP system (MACOGENIC ${ }^{\circledR}$ G2, MacoPharma). Carboxyfluorescein succinimidyl ester (CFSE) was used to monitor the inhibition of $\mathrm{T}$ cell proliferation after treatment. A proliferative $\mathrm{T}$ cell response was induced by phytohemagglutinin (PHA) in post-ECP and untreated controls. After 72 hours the 7-Aminoactinomycin (7-AAD) and CFSE reactivity of CD3 lymphocytes was analyzed by flow cytometry. With this non-radioactive method we measured the percentage of viable and dead cells and calculated the rate of $\mathrm{T}$ cell proliferation.

Results: UV-A irradiation and 8-MOP treatment resulted in augmented T cell death as shown by 7-AAD ${ }^{+}$staining independent from PHA stimulation after 72 hours $(\mathrm{p}<0.001)$. Upon PHA stimulation, $64 \pm 9 \%$ control $\mathrm{T}$ cells had divided after 72 hours, whereas through ECP the mean T cell proliferation rate was significantly reduced $(5 \pm 4 \% ; \mathrm{p}<0.001)$, the calculated inhibition was $92 \pm 5 \%$.

Conclusion: We found that due to ECP the majority of T cells had died after 72 hours and proliferation was markedly reduced. In conclusion this characterization in vitro combining a marker for cell proliferation and cell death in a single test may be a practical tool to validate the ECP procedure.

\section{TTH-V04 \\ Comparison of the apheresis devices AMICUS, Spectra OPTIA and Cobe SPECTRA in non-stimulated mononuclear cell collections}

\section{*J.C. Fischer, F. Wenzel, J.M. Rox}

${ }^{1}$ Heinrich Heine Universität Düsseldorf, Med. Fakultät, Institut für Transplnatationsdiagnostik und Zelltherapeutika, Düsseldorf, Germany

Background: Harvesting mononuclear cells (MNC) as a starting source for manufacturing therapeutic cells is one of the main indications for therapeutic apheresis - beside collection of peripheral hematopoietic progenitors. The Cobe SPECTRA (Terumo BCT) is the most commonly used device for this kind of MNC apheresis In Germany. Since this device will be discontinued, we compared its harvesting ability with that of Spectra OPTIA MNC (Terumo BCT) and AMICUS (Fenwall) for MNC collection in non-stimulated patients/ donors.

Methods: 49 healthy volunteers/ donors and 59 cancer patients underwent a total of 163 (75 resp. 88) non stimulated MNC apheresis procedures within the last two years. For the harvesting either the AMICUS device $((A), n=40)$, Spectra OPTIA MNC $((O), n=10)$ or Cobe SPECTRA $((\mathrm{S}), \mathrm{n}=113)$ was used. All apheresis were done with ACDA as the only anticoagulant with an ACDA rate up to $0.9 \mathrm{ml} \mathrm{ACDA} / \mathrm{min}$ and $1 \mathrm{BV}$. Collections were done according to manufacturer's instructions through exclusively peripheral vein access. Blood differential counts were obtained before and after apheresis and from the product itself using either a Cell
Dyn 3500 or Ruby. For the cellular fractions collection efficiency (CE) and throughput (TP) were calculated as follows: CE1 = cells harvested / cells processed; $\mathrm{TP}=$ cells harvested $/$ body weight $[\mathrm{kg}] /$ mean $\mathrm{PB}$ concentration $[/ \mu 1] /$ collection time $[\mathrm{min}]$.

Results: Patient/ donor characteristics were comparable between the (A), (O), (S) groups.

Using (S) more MNC could be harvested than using(A) (CE 57 vs 51\%, $\mathrm{p}<0.03$, stratified for donors/patients), resulting in higher MNC TP (381 vs $340, \mathrm{p}<0.02)$. While Lymphocyte (LY) CE (57\%) and TP (374) are comparable between both devices, less Monocytes (Mo) could be harvested with (A) (CE 30 vs 18\%, TP 387 vs 256). Less Granulocytes (GR, CE 1 vs $9 \%$ ) and platelets (PLT, CE $21 \%$ vs $7 \%$ ) were collected by A. When comparing (A) to (O) differences of Ly CE (57 vs 64\%) and TP (374 vs 480) reached no significance. Less Mo could be harvested with (A) (CE 18 vs 33\%, TP 256 vs 545), less PLT (CE 7 vs 24\%), but more RBC (CE 0,5 vs 0,02\%).

Accordingly (O) showed better Ly TP (480 vs 375), Mo TP (545 vs 387) than (S), but harvested less GR (CE 2 vs 9\%) and less PLT (CE 16 vs 21\%) These results were confirmed by analysis in those cases, where inter-individual pair wise comparison was possible (total 23 pairs).

Conclusion: The newer devices (AMICUS/ OPTIA) collect purer MNC products than the older SPECTRA. While Ly collection efficacy is comparable, SPECTRA and even more OPTIA collect more efficient Ly. Lowest Plt together with highest RBC contamination is seen in AMICUS, OPTIA delivers lowest RBC with intermediate PLT contamination. Thus wanted cell material composition may determine the most adequate kind of apheresis device.

\section{TTH-V05 \\ Collection of peripheral blood stem cells (PBSC) with Spectra and Amicus apheresis systems}

\section{*J. Schmidt, S. Winterfeld, H. Kroll}

Red Cross Blood Transfusion Service NSTOB, Institute for Transfusion Medicine, Dessau-Roßlau, Germany

Background: Peripheral blood stem cells (PBSC) can be collected by various automated haemapheresis systems. In the Spectra (Terumo) system the stem cell containing buffy-coat is continuously collected into the product bag. In contrast, in Amicus PBSCs are first concentrated within the centrifugation chamber and than harvested in several cycles. Here, we compared the suitability of two systems for the collection of PBSCs. Methods: A total of 104 allogeneic PBSC apheresis procedures were analyzed. PBSCs were mobilized with $10 \mu \mathrm{g} / \mathrm{kg}$ body weight G-CSF over five days split into two doses per day (only one dose on day 5). Donors were included into the evaluation when their pre-collection CD34+ cell counts $\left(0-183 \times 10^{3} / \mu \mathrm{l}\right)$ were within the $95 \%$-confidence interval of all mobilizations. Cells were harvested by Spectra $(n=72)$ and Amicus $(n=32)$ using the MNC program. The Spectra and Amicus groups did not differ in pre-collection CD34+, WBC, PLT cell counts, but differed with respect to age (34 vs. 28 years) and body weight (75.7 vs. $86.2 \mathrm{~kg}$ ). Results: The amount of CD34+ cells $\left[\times 10^{6}\right]$ collected per apheresis was $571 \pm 294$ with Spectra and $408 \pm 212$ with Amicus $(\mathrm{p}<0.01)$, MNCs $\left[\times 10^{8}\right] 506 \pm 155$ vs. $406 \pm 124(\mathrm{p}<0.01)$. The duration of donation was $265 \pm 48$ min with Spectra and $297 \pm 32$ min with Amicus $(p<0.01)$. The platelet concentration $\left[\mathrm{x} 10^{10}\right]$ in the collected preparations differed: $76 \pm$ 27 (Spectra) vs. $20 \pm 11$ (Amicus) $(\mathrm{p}<0.01)$. The platelet loss $\left[\times 10^{3} / \mu 1\right]$ in donors was $123 \pm 63$ with Spectra and $36 \pm 23$ with Amicus $(\mathrm{p}<0.01)$. In 4 cases $(6.0 \%)$ of Spectra donors the post-collection platelet count fell below $80 \times 10^{3} / \mu \mathrm{l}$ in contrast to none of the Amicus donors. The preparation volume [ml] was $236 \pm 38$ (Spectra) and $192 \pm 50$ (Amicus) $(\mathrm{p}<0.01)$. In eight cases, four each with Spectra (6.0\%) and Amicus (12.5\%) the procedure did not achieve the transplant dose of $4 \times 10^{6} \mathrm{CD} 34+/ \mathrm{kg}$ bodyweight of the recipient, so that a second separation was required.

Conclusion: Amicus can effectively be used for the collection of PBSCs. With Spectra higher amounts of stem cells were collected in less time. With Amicus, products with smaller volumes were generated. Critical post-collection platelet counts were not observed with Amicus. 
TTH-V06

\section{Effective and safe collection of peripheral blood stem cells in patients and donors using the Amicus ${ }^{\circledR}$ Separator and the Spectra Optia ${ }^{\circledR}$ Apheresis System}

\section{${ }^{*}$ A. Sputtek', C. Schubert², S. Peine ${ }^{2}$, A.W. Rowe ${ }^{3}$}

${ }^{1} \mathrm{MVZ}$ Labor Limbach Neumünster $\mathrm{GmbH}$, Neumünster, Germany 'Universitätsklinikum Hamburg-Eppendorf, Institut für Transfusionsmedizin, Hamburg, Germany

${ }^{3}$ New York University School of Medicine, New York (NY), United States

Background: Because technical support of the Cobe ${ }^{\circledR}$ Spectra Apheresis System after 2014 is uncertain, we therefore compared the efficacy of two alternative blood cell separators, i.e. the Spectra Optia ${ }^{\circledR}$ Apheresis System and the Amicus ${ }^{\circledR}$ Separator.

Methods: After mobilization using G-CSF alone or G-CSF plus Plerixafor, 36 PBSC components were collected with each machine from patients and healthy donors. The process time was adjusted to $4 \mathrm{~h}$ irrespective of the processed blood volumes. The numbers of collected CD34+ cells, CD34+ collection efficiencies, and CD34+ collection rates amounted to $386+/-413 \times 10^{6}, 64.6+/-14.6 \%, 1.55+/-1.65 \times 10^{6} / \mathrm{min}$ (Amicus) and $378+/-404 \times 10^{6}, 71.1+/-12.0 \%, 1.56+/-1.69 / \mathrm{min}$ (Optia), respectively. However, these differences were not significant $(\mathrm{P}>0.05)$. The components collected with the Amicus contained $21.3+/-8.5 \times 10^{9}$ mononuclear cells compared with $18.4+/-8.7 \times 10^{9}$, for the Optia, however, this difference was not significant as well.

Results: The Optia products showed a slightly (but not significantly) higher contamination with granulocytes $\left(11.0+/-6.9 \times 10^{9}\right)$ vs. $9.2+/-4.8$ for the Amicus. Product hematocrits due to contaminating red cells were significantly higher for the Amicus $(0.029+/-0.0161 / 1)$ in comparison to the Optia $(0.013+/-0.0191 / 1)$. On the other hand, the number of platelets contained in the Optia products was almost 4 times higher than in the case of the Amicus products $\left(2.6+/-1.5 \times 10^{11}\right.$ vs. $0.7+/-0.3 \times 10^{11}, \mathrm{P}<$ $0.0001)$. The decrease in peripheral platelet counts observed for the Amicus $(25.1+/-15.8)$ was significantly less pronounced $(\mathrm{P}<0.001)$ than in the case of the Optia $(55.9+/-38.6 / \mathrm{nl})$. The number of platelets contained in the Optia products was 4 times higher than in the case of the Amicus products $\left(2.6+/-1.5 \times 10^{11}\right.$ vs. $\left.0.7+/-0.3 \times 10^{11}\right)$.

Conclusion: The decreases of the peripheral hemoglobin concentrations after the collection compared to the starting values were not significantly different, i.e. $0.7+/-0.7 \mathrm{~g} / \mathrm{dl}$ (Amicus) vs. $1.1+/-1.8 \mathrm{~g} / \mathrm{dl}$ (Optia). Typical moderate citrate related reactions in 4 out of the 72 runs were observed; however, all reactions could be stopped by either oral or intravenous $\mathrm{Ca}$ $2^{+}$administration. Both blood cell separators demonstrated a safe and efficient performance for peripheral stem cell collections in patients and donors and, as such, are suitable for replacement of the Cobe ${ }^{\circledR}$ Spectra Apheresis System.

\section{TTH-V07}

Plasma Exchange (PE) Therapy for Rituximab (R) induced Progressive Multifocal Leukoencephalopathy (PML) in Hematologic Disorders

${ }^{*}$ S. Wienzek-Lischka ${ }^{1}$, A. Burchardt², C. Schoelz³ , H. Hackstein ${ }^{1}$, M. Rummel ${ }^{2}$, G. Bein ${ }^{1}$

${ }^{1}$ Universitätsklinikum Gießen und Marburg $\mathrm{GmbH}$, Zentrum für Transfusionsmedizin und Hämotherapie, Gießen, Germany

2Universitätsklinikum Gießen und Marburg GmbH, Medizinische Klinik, Hämatologie und Onkologie, Gießen, Germany

${ }^{3}$ Universitätsklinikum Gießen und Marburg $\mathrm{GmbH}$, Neuroradiologie, Gießen, Germany

Background: PML is a rare opportunistic disease that is most commonly associated with acquired immunodeficiency syndrome and results from reactivation of latent $\mathrm{JC}$ polyoma virus (JCV). In 2009 Carson et al. reported 57 cases after Rituximab (R) therapy in HIV negative patients. No curative therapy for PML exists, treatment is mostly supportive. In Natalizumab induced PML removal of the drug via plasma exchange is an important part of the therapy. Here we report the first successful case of $\mathrm{R}$ with a plasma exchange (PE) therapy accompanied by our CDC - based assay for monitoring efficient $\mathrm{R}$ removal from patients plasma.

Case: A 70 year old patient with a relapse of a marginal zone lymphoma was admitted with diagnosis of a $\mathrm{R}$ induced PML to our hospital. $\mathrm{He}$ was treated with Rituximab-Bendamustine therapy and Rituximab maintenance therapy. Patient achieved a good partial remission. However, 6 weeks after the last $\mathrm{R}$ administration, occurred a paraparesis of the legs and paresis of the left arm.

PML diagnosis was supported by detection of JCV with polymerase chain reaction (PCR) in cerebrospinal fluid. Total WBC was normal (6.4 G/1), but severe lymphopenia $(0.7 \mathrm{G} / 1)$ was observed. IgG, IgM and IgA levels were not measurable, B cells were not detectable, CD4-pos. lymphocyte count was low $(99 / \mu 1)$.

Results: Beside therapy with Mefloquine, Mirtazapine, IVIG were substituted. Nevertheless, R concentration was very high, therefore a PE therapy was started to remove R. PE therapy was carried out with MCS+ (HaemoneticsTM). A total blood volume of approximately 91 was processed and substituted with about 4,51 fresh frozen plasma.

Measurement of $\mathrm{R}$ level is routinely not available and no commercial kit for monitoring of a PE therapy exists. Therefore we used our CDC based assay to monitor $\mathrm{R}$ concentration in patients serum. Pre-treatment $\mathrm{R}$ level was $30.8 \mu \mathrm{g} / \mathrm{ml}$, after 7 th apheresis $6.15 \mu \mathrm{g} / \mathrm{ml}$ and at the end of PE therapy (14th apheresis) $1.83 \mu \mathrm{g} / \mathrm{ml}$. Our findings obtained with CDC-based assay were concordant with the test results received from Roche Int.

Neurologic symptoms of the patient improved within first 2 weeks. Lymphopenia disappeared and CD4 count increased.

Conclusion: To accelerate immune response, rapid elimination of $\mathrm{R}$ with PE therapy appears as a new additional and useful tool and needs further investigation. Furthermore our CDC dependent assay is a rapid and immediate available method for monitoring PE Therapy of patients with serious adverse events of $\mathrm{R}$ treatment.

\section{Poster: Therapeutische Hämapherese}

\section{TTH-P01 \\ Hemoglobin and Hematokrit during and after Preparatory Plasmaphereses without and with Saline Infusion}

\author{
*J. Evers, N. Ehren, T. Engelen, M. Hansen, K. Luethje, \\ *U. Taborski
}

DGH Plasmaspende, Aachen, Germany

Background: Although the influence of an infusion of $500 \mathrm{ml} \mathrm{NaCl} 0.9 \%$ during preparatory plasmaphereses (PP) on separated plasma was recently studied only insufficient data could be found in PubMed on changes of hemoglobin (HGB) concentration and hematocrit (HCT) in donor blood during and after PP without or with a saline infusion. The aim of this study was to evaluate the course of HGB concentration and HCT during and after PP with the Haemonetics ${ }^{\circledR}$ plasma collecting system 2 (PCS2) without saline infusion and in $\mathrm{PP}$ with an infusion of $500 \mathrm{ml} \mathrm{NaCl} 0.9 \%$. Methods: After informed consent 32 frequent donors of plasma (17 m, 15 f) were studied in a crossover design on a day (A) without $\mathrm{NaCl}$ during $\mathrm{PP}$ and on another day (B) with infusion of $\mathrm{NaCl} 0.9 \% 500 \mathrm{ml}$ during PP. The average age of the participants was $30 \pm 11$ years, average weight was $70.4 \pm 8.9 \mathrm{~kg}$, and average volume of separated plasma was $11.7 \pm 1.1 \mathrm{ml} /$ $\mathrm{kg}$. Complete blood counts including concentrations of HGB and HCT in donor blood were analysed by the Sysmex KX-21N apparatus. The values of $\mathrm{HGB}$ and $\mathrm{HCT}$ before PP were set to $100 \%$. After PP changes of HGB and HCT were calculated in percent immediately, after $24 \mathrm{~h}$, and after $72 \mathrm{~h}$.

Results: The results are summarized in tables 1 and 2. Data are reported as average $\pm \mathrm{SD}$. 
Table 1. Results of HGB ( $\mathrm{g} \%$ )

\begin{tabular}{|l|l|l|}
\hline & without $\mathrm{NaCl}(\mathrm{A})$ & with $\mathrm{NaCl}(\mathrm{B})$ \\
\hline HGB before PP & $14.3 \pm 1.2$ & $14.4 \pm 1.0$ \\
\hline HGB after PP & $15.9 \pm 1.3$ & $15.1 \pm 0.9$ \\
\hline HGB after $24 \mathrm{~h}$ & $14.9 \pm 1.1$ & $14.8 \pm 1.1$ \\
\hline HGB after $72 \mathrm{~h}$ & $14.2 \pm 1.1$ & $14.2 \pm 1.1$ \\
\hline
\end{tabular}

Table 2. Results of HGT (\%)

\begin{tabular}{|l|l|l|}
\hline & without $\mathrm{NaCl}(\mathrm{A})$ & with $\mathrm{NaCl}(\mathrm{B})$ \\
\hline HGT before PP & $42.9 \pm 3.2$ & $42.9 \pm 2.8$ \\
\hline HGT after PP & $47.8 \pm 3.8$ & $45.1 \pm 2.7$ \\
\hline HGT after $24 \mathrm{~h}$ & $44.5 \pm 2.7$ & $43.8 \pm 2.9$ \\
\hline HGT after $72 \mathrm{~h}$ & $42.4 \pm 3.1$ & $42.3 \pm 2.9$ \\
\hline
\end{tabular}

There were highly significant increases $(p<0.001)$ of HGB by $11.2 \pm$ $4.0 \%$ and HCT by $11.6 \pm 3.9 \%$ in donor blood during PP without saline infusion. The difference between the two samples without $\mathrm{NaCl}$ and with $\mathrm{NaCl}$ was highly significant, too $(\mathrm{p}<0.001)$. After $24 \mathrm{~h}$ and after $72 \mathrm{~h}$ all differences were reduced.

Conclusion: In this study, we observed highly significant changes of HGB and HCT in donor blood during PP. To avoid adverse reactions we recommend an infusion of $500 \mathrm{ml} \mathrm{NaCl} 0.9 \%$ during PP to all donors.

\section{TTH-P02}

\section{White Blood Cells and Platelets during and after Preparatory Plasmaphereses without and with Saline Infusion}

*J. Evers, N. Ehren, T. Engelen, M. Hansen, K. Luethje, *U. Taborski DGH Plasmaspende, Aachen, Germany

Background: Recently we studied the course of hemoglobin (HGB) concentration and hematocrit (HCT) in donor blood during and after preparatory plasmaphereses (PP) without and with saline infusion. Additionally, we like to document the course of white blood cells (WBC) and platelets (PLT) in donor blood during and after PP with Haemonetics ${ }^{\circledR}$ plasma collecting system 2 (PCS2) without saline infusion and in PP with an infusion of $500 \mathrm{ml} \mathrm{NaCl} 0.9 \%$.

Methods: After informed consent 32 frequent donors of plasma $(17 \mathrm{~m}, 15$ f) were studied in a crossover design on a day (A) without $\mathrm{NaCl}$ during PP and on another day (B) with infusion of $\mathrm{NaCl} 0.9 \% 500 \mathrm{ml}$ during PP. The average age of the participants was $30 \pm 11$ years, average weight was $70.4 \pm 8.9 \mathrm{~kg}$, and average volume of separated plasma was $11.7 \pm 1.1 \mathrm{ml} /$ $\mathrm{kg}$. Complete blood counts including WBC and PLT in donor blood were analysed by the Sysmex KX-21N apparatus.

Results: The results of WBC and PLT are summarized in tables 1 and 2. Data are reported as average $\pm \mathrm{SD}$.

Table 1. Results of WBC $\left(\times 10^{3} / \mu \mathrm{L}\right)$

\begin{tabular}{|l|l|l|}
\hline & without $\mathrm{NaCl}(\mathrm{A})$ & with $\mathrm{NaCl}(\mathrm{B})$ \\
\hline WBC before PP & $7.3 \pm 1.5$ & $7.2 \pm 1.2$ \\
\hline WBC after PP & $8.1 \pm 2.0$ & $7.5 \pm 1.4$ \\
\hline WBC after 24h & $8.8 \pm 2.3$ & $7.8 \pm 1.8$ \\
\hline WBC after $72 \mathrm{~h}$ & $6.6 \pm 2.1$ & $7.3 \pm 2.0$ \\
\hline
\end{tabular}

Table 2. Results of PLT $\left(\times 10^{3 / \mu \mathrm{L})}\right.$

\begin{tabular}{|l|l|l|}
\hline & without $\mathrm{NaCl}(\mathrm{A})$ & with $\mathrm{NaCl}(\mathrm{B})$ \\
\hline $\mathrm{PLT}$ before PP & $243 \pm 53$ & $250 \pm 50$ \\
\hline $\mathrm{PLT}$ after PP & $242 \pm 51$ & $234 \pm 45$ \\
\hline $\mathrm{PLT}$ after $24 \mathrm{~h}$ & $248 \pm 47$ & $251 \pm 44$ \\
\hline PLT after $72 \mathrm{~h}$ & $248 \pm 49$ & $242 \pm 47$ \\
\hline
\end{tabular}

There was a significantly different increase of WBC $(\mathrm{p}<0.01)$ by $21.1 \pm 19.5 \%$ in donor blood $24 \mathrm{~h}$ after PP without saline infusion. Absolute values of WBC were in normal range and the change was unspecified. There were no significantly different changes of PLT during or after PP without or with saline infusion during PP. Conclusion: We observed a significantly different increase of WBC within the normal range in donor blood $24 \mathrm{~h}$ after PP without saline infusion. The clinical importance of this finding remains to be clarified. As there were no significantly different changes of WBC $(\mathrm{p}>=0.05)$ or PLT during or after PP with saline infusion we recommend an infusion of $500 \mathrm{ml}$ $\mathrm{NaCl} 0.9 \%$ during PP to all donors.

\section{TTH-P03}

\section{Stem cell collection efficiency of Spectra Optia is superior to COBE Spectra}

\section{${ }^{*}$ L. Goudeva ${ }^{1}$, S. deReys ${ }^{2}$, R. Blasczyk ${ }^{1}$, H.-G. Heuft ${ }^{1}$}

${ }^{1}$ Medizinische Hochschule Hannover, Institut für Transfusionsmedizin, Hannover, Germany

${ }^{2}$ Terumo BCT, Zaventem, Belgium

Background: To compare the performance of the new Spectra Optia with the well established COBE Spectra apheresis system in allogeneic stem cell donors.

Methods: In 2012, we conducted a prospective study including 23 Spectra Optia runs (software version 7.1, 20 donors) and 13 COBE Spectra runs (software version 4.7, 8 donors) Donor blood volume was lower in the Spectra Optia $(5.0 \pm 0.6 \mathrm{~L})$ vs Spectra $(5.6 \pm 0.8 \mathrm{~L})$ donors, but age, gender, haematological pre- and post-counts (WBC, Gran\%, Hct, Hb, Plt) and the median target cell dose $\left(5 \times 10^{6} \mathrm{CD} 34^{+}\right.$cells $\left./ \mathrm{kg}\right)$ were not different. All donors had a peripheral venous access.

Results: Partly because of lower inlet flow rates, a lower blood volume was processed on the Spectra Optia $(10.7 \pm 2.4 \mathrm{~L}$ vs $16.2 \pm 2.9 \mathrm{~L}$; P+cells per $\mathrm{kg}$ of the recipients' body weight were harvested with the Spectra Optia $\left(6.25 \pm 5.37\right.$ vs $4.12 \pm 2.74 \times 10^{6} / \mathrm{kg}$, ns $)$. The Spectra Optia collected $\mathrm{CD} 34^{+}$cells with a significantly higher collection efficiency than the COBE Spectra ( $53 \pm 11 \%$ vs $39 \pm 10 \% ; \mathrm{P}=0.007)$. We did not find a significant difference in the product volume. In fact, as cell concentrations were higher in the Spectra Optia, the adjusted product volume was higher in the COBE Spectra ( $382 \pm 75 \mathrm{~mL}$ vs $456 \pm 53 \mathrm{~mL}$; $\mathrm{p}=0.01)$. We performed linear regression analysis to estimate prediction of $\mathrm{CD} 34^{+}$target cell dose per liter processed based on $\mathrm{CD} 34+$ pre-counts (see graph). High correlations were found for both devices (Spectra Optia $R^{2}=0.7253$; COBE Spectra $\mathrm{R}^{2}=0.8203$ ).

Conclusion: For a target dose of $5 \times 10^{6} \mathrm{CD} 34^{+}$cells $/ \mathrm{kg}$ and a median pre-count of $58 \mathrm{CD} 34^{+}$cells $/ \mu \mathrm{L}$, a process volume of 9.9 liter blood is predicted on the Spectra Optia versus 17.8 liter on the COBE Spectra. Overall, because of the higher collection efficiency of the Spectra Optia, less blood needs to be processed to obtain the same target CD $34^{+}$dose.

Fig. 1

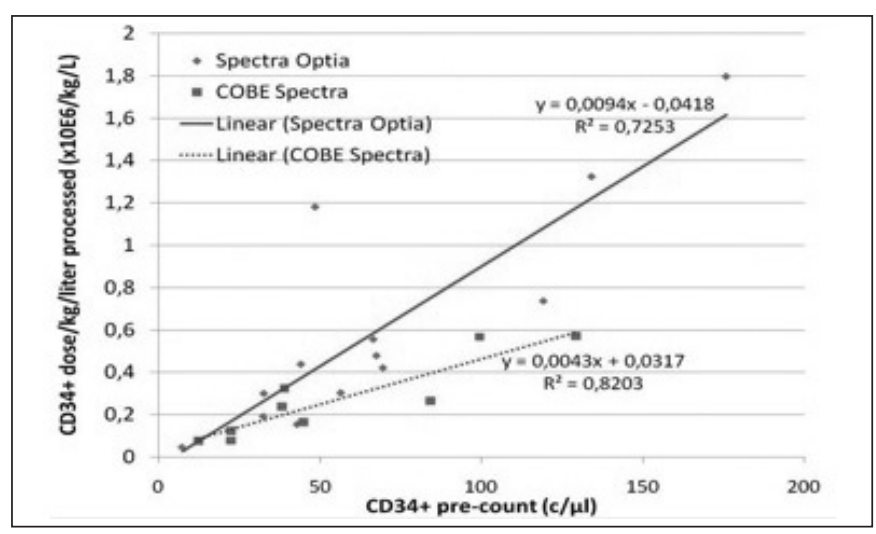


TTH-P04

\section{Evaluation of plasmapheresis performed by Autopheresis $C$ and Trima Accel from a donor, product, operator, and regulator perspective}

\section{Räber', *S. Fontana'2, S. Thomas², A. Irene'2, A. Tschaggelar²}

${ }^{1}$ Inselspital Bern, Hämatologie, Bern, Switzerland

${ }^{2}$ Blutspendedienst SRK, Bern, Switzerland

Background: Plasmapheresis with Autopheresis C (A) is well-established since many years and allows collection of up to $600 \mathrm{~mL}$ plasma from every donor, as required by the Swiss regulations. Recently, the Trima Accel (T) system has been developed for the collection of plasma up to $15 \%$ of the donor blood volume, according to the Council of Europe recommendations. We evaluated the new $\mathrm{T}$ plasmapheresis procedure and compare it to A from a donor, product, and operator perspective, also considering the current requirements for transfusion plasma and for pathogen reduction with amotosalen and UVA.

Methods: In 57 consecutive apheresis procedures donors evaluated their first donation on $\mathrm{T}$ for comfort and side effects by a standardized questionnaire, and compared it with their previous donation on A. Collection data (duration and collected volume), cell counts, protein, IgG, and coagulation factors (the last in pools of 5 donations) were measured for 20 donations, and compared to 20 procedures performed with Autopheresis C. Operators evaluated the 2 systems by a standardized questionnaire.

Results: Donors:53 available evaluations showed for A and T a 4 (good) to 5 (very good) score for comfort and side effects. The perception of citrate toxicity was comparable (better tolerance with A $2 / 53$, with $\mathrm{T}$ $7 / 53, \mathrm{p}>0.05)$, with $\mathrm{T}$ the general comfort was higher (A 1/53, T 18/53, $\mathrm{p}<0.01)$, and the donations slightly shorter $(8 \mathrm{~min}, \mathrm{p}<0.01)$. Mean cell counts:comparable for both platelets (A and T $1 \times 10^{9} / \mathrm{L}$ ) and leukocytes (A and $\mathrm{T}<0.01 \times 10^{6} /$ unit), however lower for erythrocytes in $\mathrm{T}$ (A $\left.0.47 \times 10^{9} / \mathrm{L}, \mathrm{T} 0.002 \times 10^{9} / \mathrm{L}, \mathrm{p}<0.01\right)$. Proteins and coagulation factors: trend for higher values in A $(10 \%$ activity differences, $\mathrm{p}=0.07)$, excepted for measures of coagulation activation $(\mathrm{p}>0.1)$.

Conclusion: Both $\mathrm{A}$ and $\mathrm{T}$ are safe and effective plasma collection systems, fulfilling the current legal requirements and the requirements for the pathogen reduction process with amotosalen and UVA, in spite of higher erythrocyte counts in A. The higher donor comfort with $\mathrm{T}$ could be explained by the donor volume substitution and the shorter donation time. The dilution could also explain the trend to lower protein and coagulation factor levels of T, as coagulation activation markers were normal. Shorter procedure times and lower work load for operators may compensate for the higher cost of $\mathrm{T}$.

\section{TTH-P05}

\section{Higher HSC collection efficiency on Spectra Optia}

\section{${ }^{*}$ E. Becker ${ }^{1}$, K. Wuttig ${ }^{1}$, E. Klatt', A. Parkner ${ }^{1}$, F. Heidel',} M.U. Heim

${ }^{1}$ Otto-v.-Guericke-Universität , Institut für Transfusionsmedizin und Immunhämatologie mit Blutbank, Magdeburg, Germany

${ }^{2}$ Otto-v.-Guericke-Universität, Klinik für Hämatologie und Onkologie, Magdeburg, Germany

Background: Spectra Optia, the novel platform for moblized stem cell collection developed by TerumoBCT, has recently been introduced in our center. We evaluated the ability of the Spectra Optia to collect HSC (Hematopoietic Stem Cells). The objective was to compare CD34+ collection efficiency, product and procedure characteristics using Spectra Optia versus COBE Spectra.

Methods: Collection characteristics from 10 allogenic stem cell apheresis procedures performed with Spectra Optia were analyzed and compared to 17 allogenic procedures with COBE Spectra in 2011-2012. HSC collections were performed according to our routine standards. Data are given as median (range) and statistically significant improvements measured by a Mann-Whitney test are indicated by (*), p-value attached.
Results: Product parameters and efficiencies are summarized in Table 1 . CD34+ cell precounts, body weight of recipients, total volume of blood processed and product volume were comparable.

CD34+ collection efficiency was significantly higher $(p=0.0127) 6$ with Spectra Optia compared to COBE Spectra (Table 1) and was positioned approximately $15 \%$ higher across the range of CD34+ cells precount (Fig 1.). The correlation between apheresis CD34+ cell precounts and cell dose collected/L of blood was quite tight on both devices $\left(\mathrm{R}^{2}=0.88\right.$ Spectra Optia; $\mathrm{R}^{2}=0.89$ COBE Spectra), allowing prediction of CD34+ collected (Fig 2). We also observed that for a specific CD34+ cell precount, Spectra Optia collected $30 \%$ more cells/L of blood processed.

Conclusion: Our preliminary data showed that across a wide range of initial circulating CD34+ cells, Spectra Optia delivered a very good CD34+ collection efficiency, higher than with the COBE Spectra device. As CD34+ dose per L of blood processed was strongly correlated to pre-apheresis CD34/ $\mu \mathrm{L}$, it allows prediction of CD34+ cells collection. Spectra Optia appears a suitable replacement for COBE Spectra for HSC collection.

\section{Table 1.}

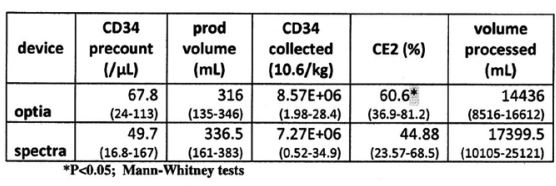

Fig 1

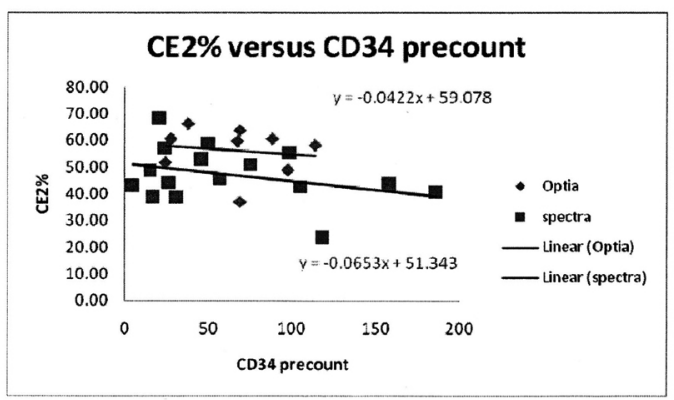

Fig 2

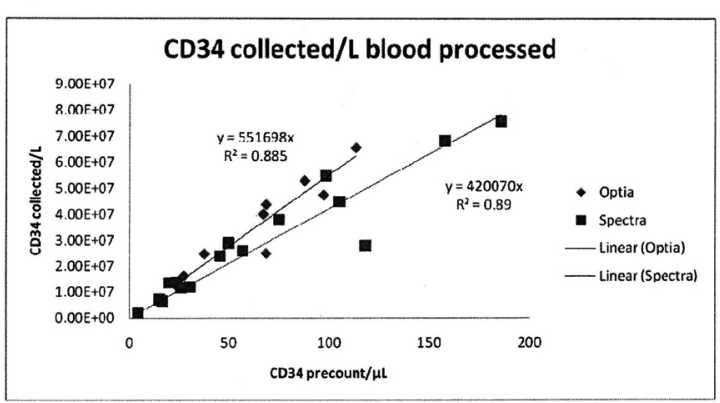

TTH-P06

Procedural blood flow in peripheral stem cell apheresis is dependent on the peripheral vein diameter

\section{${ }^{\star}$ F. Wenzel, J. Noriega, J. Rox, J. Fischer, G. Giers}

Universitätsklinik Düsseldorf, IHTM, Düsseldorf, Germany

Background: In donors undergoing a peripheral stem cell (PBSC) apheresis the amount of collected CD34+-cells is dependent on the processed volume. Normally peripheral cubital veins are adequate for generating an acceptable blood flow to guarantee an attainable time duration. But in some cases a peripheral access is not achievable, and a central venous catheter (CVC) is required implicating additional adverse risks like carotid artery or lung injury. Therefore in this study we desrcribed the cor- 
relation between veins and procedural blood flow in PBSC apheresis and tried to identify limitations for the use of peripheral veins.

Methods: 40 PBSC donations (allogenic $n=20$, autologous $n=20$ ) were examined using 16 Gauge catheters (Vasofix, Fa. Braun, Germany). Venous diameters were determined by compression ultrasonography (Sonoline Adara, Fa. Siemens, Germany) at a venous congestion of $40 \mathrm{mmHg}$. Blood pressure and heart rate were measured, and procedural parameters like blood flow were calculated. Additionally, correlations between inlet pressure and procedural flow were determined for the respective venous catheter.

Results: Inlet pressures were dependent on the fluid viscosity and venous diameters: at a constant procedural blood flow of $75 \mathrm{~mL} / \mathrm{min}$ a mean inlet pressure of about $60 \mathrm{mmHg}$ was required using whole blood and about 10 $\mathrm{mmHg}$ using $\mathrm{NaCl}$ solution. Veins with cross-sectional areas $<25 \mathrm{~mm}^{2}$ showed a mean inlet pressure of $84 \mathrm{mmHg}$ accompanied by a mean blood flow of about $50 \mathrm{~mL} / \mathrm{min}$ in comparison to veins with cross-sectional areas $>25 \mathrm{~mm}^{2}$ showing a mean inlet pressure of $57 \mathrm{mmHg}$ resulting in higher blood flows of about $100 \mathrm{~mL} / \mathrm{min}$. Additionally, an elevation of the mean arterial blood pressure (MAP) resulted in an increase in procedural blood flow $(67 \pm 19 \mathrm{~mL} / \mathrm{min}(\mathrm{MAP}<100 \mathrm{mmHg})$ vs. $76 \pm 20 \mathrm{~mL} / \mathrm{min}$ (MAP > $100 \mathrm{mmHg})$ ).

Conclusion: It could be derived from the observed correlations between venous diameters and inlet procedural pressures that veins showing a cross-sectional area $<15 \mathrm{~mm}^{2}$ could not generate an acceptable blood flow for PBSC apheresis. This may be a clear indication for the application of central venous catheters. Additionally, procedural blood flow was dependent on the mean arterial pressure.

\section{TTH-P07}

\section{The acute effect of granulocyte colony-stimulating factor on bone turnover}

S. Oczko, *N. Sareban, C. Drexler, S. Sipurzynski, A. Fahrleitner-Pammer, H. Dobnig, T. Pieber, G. Lanzer, K. Amrein Medizinische Universität Graz, Graz, Austria

Background: Granulocyte colony-stimulating factor (G-CSF) is widely used to mobilize peripheral blood stem cells (PBSC) and enable allogeneic or autologous PBSC collection by apheresis. Although bone pain is a common adverse event following G-CSF treatment, little is known on its effect on bone metabolism. To determine the association between treatment with G-CSF and bone resorption/formation, we examined these markers during mobilization in 18 patients.

Methods: Markers of bone turnover (OC, osteocalcin, $\beta$-CTx, bALP, C-terminal telopeptide of type I collagen, bone specific alkaline phosphatase, TRAP, tartrate resistant acid phosphatase) and mineral metabolism were assessed in 18 adult patients with haematological malignancy who received G-CSF for autologous PBSC collection. Analyses were repeated after G-CSF stimulation. Subjective bone pain assessed by visual analogue scale (range 0: no pain - 10: worst imaginable pain) and use of analgetics was recorded. Patients with advanced chronic kidney disease (glomerular filtration rate $<30 \mathrm{ml} / \mathrm{min}$ ) were excluded from analysis.

Results: 18 subjects were included ( 10 men, 8 women, mean age $48 \pm 11$ years, BMI $24.5 \pm 2.9 \mathrm{~kg} / \mathrm{m}^{2}$ ). B-CTX and bALP were elevated already at baseline. OC, bALP and TRAP were significantly altered by G-CSF, while $B$-CTX, calcium and serum phosphate levels remained unchanged (Table 1). Bone pain was $4.5 \pm 2.9$ points (range $0-8$ ) and half of all patients required analgetics. No significant correlation between bone pain and bone turnover markers or CD34+ cell yield was observed.

Conclusion: Our results demonstrate that high-dose G-CSF acutely affects bone metabolism. The clinical relevance of these finding is to date unclear, but further research is warranted to confirm our findings and ascertain long-term skeletal health in this vulnerable population.

\begin{tabular}{|l|c|c|c|}
\hline $\begin{array}{l}\text { Biochemical marker, } \\
\text { reference range }\end{array}$ & Before G-CSF & After G-CSF & $\mathbf{P}$ \\
\hline$\beta$-CTx, 0.06-0.35 ng/mI & $0.40 \pm 0.34$ & $0.42 \pm 0.32$ & 0.57 \\
\hline Osteocalcin, 1.0-35.0 ng/mI & $25.3 \pm 18.7$ & $16.5 \pm 10.0$ & $\mathbf{0 . 0 0 7}$ \\
\hline bALP, 7.5-20.6 U/l & $22.8 \pm 17.7$ & $33.7 \pm 17.9$ & $\mathbf{0 . 0 0 2}$ \\
\hline TRAP, 2.59-4.03 $\mu \mathrm{g} / \mathrm{l}$ & $3.41 \pm 1.57$ & $2.78 \pm 1.15$ & $\mathbf{0 . 0 0 2}$ \\
\hline Total calcium, 2.25-2.65 mmol/l & $2.30 \pm 0.14$ & $2.31 \pm 0.19$ & 0.88 \\
\hline Ionized calcium, 1.15-1.35 mmol/l & $1.15 \pm 0.06$ & $1.16 \pm 0.09$ & 0.76 \\
\hline Serum phosphate, 2.6-4.5 m gl/dl & $3.3 \pm 0.6$ & $2.9 \pm 0.7$ & 0.08 \\
\hline
\end{tabular}

Table 1: Biochemical changes before and after G-CSF administration

\section{TTH-P08}

\section{Results of sterility testing of photopheresis products}

\section{${ }^{*}$ E. Richter, A. Brosig, V. Hähnel, F. Dormann, N. Ahrens}

Universitätsklinikum Regensburg, Institut für Klin. Chemie und Laboratoriumsmedizin, Regensburg, Germany

Background: Extracorporeal photopheresis (ECP) is an apheresis procedure in which cell suspensions from leukapheresis are treated with 8-methoxypsoralen (8-MOP) followed by UVA-irradiation and finally returned to the patient. The treated lymphocytes become apoptotic and reduce immunologic activity. Sterility of the cell suspensions for these immunosuppressed patients is therefore of special importance. However, not much is known about sterility in photopheresis.

Methods: All photopheresis treatments at University Hospital Regensburg are probed for anaerobic and aerobic sterility (Bactec, BD) after the addition of 8-MOP and before UVA-irradiation. All procedures were included between 26.09.2012 and 31.03.2013. In addition, peripheral blood was tested before photopheresis for sterility between 4.12 .2012 and 31.03.2013.

The suitability of the sterility testing method was validated by spiking leukapheresate samples containing 8-MOP with Candida albicans, Staphylococcus aureus, Bacillus subitlis, or Clostridium sporogenes at $30 \mathrm{CFU}$ each.

Results: The validation of the sterility testing method revealed delayed growth for Candida albicans. Detection of Staphylococcus aureus, Bacillus subitlis and Clostridium sporogenes was not altered by 8-MOP.

Altogether, we could include 336 photopheresis procedures of $27 \mathrm{pa}-$ tients. Of these, 9 apheresates of 3 different patients were non-sterile $(7 \times$ Staphylococcus epidermidis, $1 \times$ E. faecium, $1 \times$ Capnocytophaga ochracea). In addition, sterility testing was positive in peripheral blood on 4 occasions $(2 \times$ Staphylococcus epidermidis, $1 \times$ Propionibacterium acnes, $1 \times$ aerobic spore forming bacilli). Positive sterility testing affected 6 of the 27 patients ( 1 patient was once positive in peripheral blood and once positive in the cell suspension, 2 patients were positive only in cell suspensions, and 3 patients were positive only in peripheral blood).

Conclusion: The frequency of positive findings is conspicuous in these immunocompromised patients ( $8 \%$ of the cell suspensions, $22 \%$ of the patients). Particularly, positive sterility testing from peripheral blood may be interpreted as bacteriemia in these patients. In addition, contamination during venipuncture and/or during manipulation of the cell suspension can not be excluded despite applying clinical sterile handling methods. All cases resulted in a change of antibiotics, revision of the central line, or adaptation of the photopheresis treatment frequency. 


\section{TTH-P09}

\section{CRP-response as parameter for successful granulocyte transfusion}

\author{
${ }^{*} K$. Dullinger, G. Schmitz, E. Holler, N. Ahrens \\ UKR, Transfusionsmedizin, Regensburg, Germany
}

Background: Granulocyte transfusion (GK-Tx) is an established treatment for neutropenic patients with progressive infections despite extensive medical treatment. There is insufficient knowledge about parameters for the early identification of successful GK-Tx.

Methods: This retrospective study included patients from January 2011 to December 2012 with a diagnosis of acute leukemia and planned/performed allogenic blood stem cell transplantation with prolonged neutropenia, for whom GK-Tx was indicated due to severe and refractory fungal infection. Overall 54 healthy and unrelated donors were recruited and mobilized with dexamethasone and a single dose of G-CSF. Depending on the donor's blood results, the apheresis was performed only once or on two consecutive days with the COBE Spectra. The cell separator was used with the MNC-apheresis kit for the PMN-program. Time between indication and first GK-Tx was typically 2-3 days.

Results: This study included 99 GK-Tx in 17 patients. The GKs had a median of $310 \mathrm{ml}(173-364 \mathrm{ml})$ with $5,55 \times 10^{10}$ granulocytes/unit (median; $1,26-12,58$ ) corresponding to $7,4 \times 10^{8}$ granulocytes $/ \mathrm{kgBW}$ (median; $1,5-18,4) .9$ patients survived the fungal infection and showed decreasing CRP-levels in the first $48 \mathrm{hrs}$ following the beginning of the GK-Tx. 8 patients died during the observation period ( 2 due to the progress/relapse of the underlying disease, 2 due to refractory gastrointestinal GvHD grade III or IV, 3 due to the progress of the underlying fungal infection). 1 patient was lost for follow-up after discharge into palliative care shortly after the last GK-Tx. GK-Tx was not able to prevent the progress of the fungal infection in only 4 out of 17 patients. Death was registered within a median of 7 days after the last GK-Tx (5-25 days). There was no decrease in CRP in the patients with lethal outcome.

Conclusion: Our data suggest that the CRP value is a valuable parameter to predict the success of granulocyte transfusions, a decrease in CRP levels supports continuation of further transfusions. Overall, the outcome of patients with refractory fungal infections compares favorable to historical controls.

\section{TTH-P10}

\section{Kinetics of CD34+ progenitors in peripheral blood during stem cell apheresis}

\section{*J.M. Rox, F. Wenzel, J.C. Fischer}

University Hospital, Inst. for Transplantation Diagnostics and Cellular Therapeutics, Düsseldorf, Germany

Background: During stem cell apheresis CD34+ progenitor cells are recruited from extravascular sites. In large-volume apheresis the number of CD34+ cells collected can exceed the number calculated to be in the peripheral blood at the begin of the procedure by several times. The kinetics of the $\mathrm{CD} 34+$ progenitors in the peripheral blood during apheresis may be dependent on the type of $\mathrm{CD} 34+$ cell mobilization. In addition, the amount of exhaustion of the circulating CD34+ cell pool during apheresis is not known. Methods: 28 apheresis procedures were investigated. Peripheral blood CD34+ cell counts were done before, at midpoint (after processing $50 \pm 11 \%$ of the final processed volume), and after collection using the ISAHGE method. Allogeneic donors $(n=14)$ were treated with lenograstim $(10 \mu \mathrm{g} / \mathrm{kg}$ for $5 \mathrm{~d})$ for $\mathrm{CD} 34+$ cell mobilization, autologous patients $(n=14)$ with a combination of chemotherapy and various rh-G-CSFs. Two patients received in addition plerixafor.

Results: Peripheral blood CD34+ cell concentrations decreased from 66/ $\mu 1$ (median; range: 9 to 507) pre apheresis to $36 / \mu 1$ ( 8 to 337) at midpoint (58 $\pm 15 \%$ compared to pre apheresis counts), and to $32 / \mu 1(6-237)$ or $(50 \pm 12 \%)$ post apheresis. In 8 out of 14 autologous patients and 9 out of 14 allogeneic donors the level of CD34+ cells dropped more than 10 per cent between midpoint and post apheresis. An increase of more than 10 per cent was observed in two autologous patients only. The procedures were subgrouped according to the processed TBV at midpoint (group 1 with $<2$ TBV $(n=17)$, group $2 \geq 2$ TBV $(n=11))$. A significant decrease of CD34+ cell counts between midpoint and post apheresis could be observed in group $1(0,58 \pm 0,12$ vs. $0,50 \pm 0,11 ; p)$ but not in group 2 $(0,58 \pm 0,19$ vs. $0,51 \pm 0,13 ; p$

Conclusion: In almost all procedures described here peripheral blood CD34+ cell kinetics during apheresis can be described by two phases. The starting continuous decrease of peripheral progenitor levels is followed by a plateau phase where the hematopoietic progenitor cells counts remain nearly stable over time, independent from the kind of mobilization. These data underline that large-volume apheresis is effective. Analysis of larger cohorts will be needed to identify donors who have a more than average decrease in peripheral blood CD34+ cell counts during apheresis and thus would not profit from large- volume apheresis.

\section{Oral Abstract Session: Zelltherapie \& Gewebe}

\section{ZTH-V01 \\ Validation of Serological Testing for Anti-Treponema pallidum from Postmortem Blood}

\author{
${ }^{*}$ U. Kalus ${ }^{1}$, I. Wilkemeyer ${ }^{1}$, A. Pruß ${ }^{1}$, G. Caspari ${ }^{1,2}$
}

${ }^{1}$ Charité-Universitätsmedizin Berlin, Institut für Transfusionsmedizin, Berlin, Germany

${ }^{2}$ Laborärztliche Arbeitsgemeinschaft für Diagnostik und Rationalisierung e.V., Berlin, Germany

Background: Serological testing for anti-HIV1/2, HBsAg, anti-HBc, anti-HCV and anti-Treponema pallidum is mandatory for the viral safety of tissue donations. In accordance with EU directives, postmortem blood may be used, if premortem samples are not available. However, most CE-marked infectious disease test equipment is not validated for testing postmortem blood. Therefore, the Paul-Ehrlich-Institut recommends the validation of all test systems used for serological investigation of postmortem blood samples. We present the validation of testing for antibodies against Treponema pallidum.

Methods: Samples of postmortem sera and samples of both pre- und postmortem sera were obtained from cornea donors. The postmortem blood samples were collected between 11 and 58 hours postmortem. Immediately after standardized blood sampling (skin disinfection, puncture of the subclavian vein with sterile equipment), the samples were centrifuged at $3.000 \mathrm{~g}$ for 15 minutes and divided in three portions and frozen at $-30{ }^{\circ} \mathrm{C} \pm 5{ }^{\circ} \mathrm{C}$. After thawing, one portion was tested unspiked for anti-T. pallidum on the Siemens-BEP-III-System and the remaining two portions of each sample were spiked with different anti-Treponema pallidum-positive standards of the National Institute for Biological Standards and Control (NIBSC Code 05/132 (WHO International Standard 1 st IS for human syphilitic plasma IgG and IgM) and NIBSC Code 10/B590 (Anti-Syphilis Quality Control Reagent Sample 2) in concentrations which give low and high positive results at the respective dilution.

Results: All premortem samples obtained up to 7 days before death were unequivocally negative in all tests. Two of the postmortem samples were false positive. Both samples were visibly hemolytic. Of the negative post postmortem sera, none of the spiked samples were false negative after 0 , 24 and 60 hours, respectively. Furthermore, no differences depending on the postmortem blood collection time, the age or sex could be demonstrated. The optical density of the samples did not change noticeably with increasing postmortem removal time

Conclusion: There is no indication that postmortem samples give false negative or false positive results with the test system and test kits used. in cases of low hemolysis. The procedure described might serve as a model for validating other test kits on postmortem samples. 


\section{ZTH-V02}

\section{Orthobiology and Tissue Engineering-Cartilage regeneration in articular joints}

\author{
${ }^{*}$ M. Endres, J.P. Krüger, U. Freymann, K. Neumann, C. Kaps \\ TransTissue Technologies $\mathrm{GmbH}$, Forschung und Entwicklung, Berlin, \\ Germany
}

Background: Articular cartilage has a limited self-healing capacity. Cartilage lesions are associated with pain, blocking, reduced mobility and have been shown to progress to severe degeneration and osteoarthritis. Debridement, microfracture or osteochondral graft transfer are common repair procedures often leading to unsatisfactory results. Over the last 20 years Tissue Engineering research has led to the development of Advanced Medicinal Therapy Products (ATMPs) for cartilage repair. Methods: For the production of ATMPs, a small biopsy from the patient's own cartilage is taken and chondrocytes are isolated under clean room conditions. These chondrocytes are expanded in vitro using serum supplemented medium to obtain a sufficient amount of cells to cover the cartilage defect. The expanded cells are combined with fibrin sealant and immersed in resorbable polymer scaffolds made of polyglycolic acid (PGA) $\left(\right.$ BioSeed $\left.^{\circledR}-\mathrm{C}\right)$. The 3D cell transplant is placed into the cartilage defect and fixed using sutures, resorbable pins or fibrin sealant.

Results: The isolation of chondrocytes from cartilage biopsies and subsequent cell expansion in vitro leads to an approx. 50 fold increase in cell number. During expansion phase, chondrocytes lose their typical function. Culturing dedifferentiated chondrocytes in a 3D environment induces cell redifferentiation which is expressed in an increase of collagen type II transcription. Clinically, transplantation of autologous chondrocytes in PGA scaffolds showed good to excellent results as determined by MRI and patient questionnaires. For chondrocyte culture, supplementation of the culture media with autologous blood serum is essential. Therefore, serum is obtained from autologous blood donations either directly in the operating room or by the transfusion medicine. Also the use of platelet rich plasma (PRP) together with chondrocyte transplants has shown promising results.

Conclusion: The use of autologous chondrocyte/PGA transplants (BioSeed $\left.^{\circledR}-C\right)$ is a therapeutic option for the treatment of traumatic and focal degenerative cartilage defects. The combination of 3D scaffold technology together with ATMPs improves handling, transplant fixation in the cartilage defect and extracellular cartilaginous matrix production. From a regulatory point of view, the preparation of blood/marrow-derived cells or PRP directly in the operating room may be considered a critical step.

\section{ZTH-V03}

\section{HLA silenced plateletes from engineered stem cells overcome platelet refractoriness in vivo}

\section{Gras ${ }^{1}$, R. Antarianto ${ }^{1}$, L. Goudeva ${ }^{1}$, S. Immenschuh ${ }^{1}$, B. Eiz-Vesper', K. Schulze'2, C. Guzmann ${ }^{2}$, *R. Blasczyk', C. Figueiredo ${ }^{1}$}

${ }^{1}$ Medizinische Hochschule Hannover, Institut für Transfusionsmedizin, Hannover, Germany

${ }^{2}$ Helmholtz Zentrum, Braunschweig, Germany

Background: Refractoriness to platelet (PLT) transfusion caused by alloimmunization against HLA class I antigens constitutes a significant clinical problem. Thus, it would be desirable to have PLT units devoid of HLA antigens. Previously, we showed that the in vitro generation of HLA class I-silenced (HLA-universal) PLTs by RNA interference from genetically engineered stem cells is feasible.

Methods: Here, we assessed the functionality of HLA-silenced PLTs and their ability to escape HLA antibody-mediated elimination in vitro and in vivo. Platelet activation in response to ADP and thrombin were assessed in vitro. The immune-evasion capability of HLA-universal megakaryocytes (MKs) and PLTs was tested in lymphocytotoxicity assays using anti-HLA antibodies. To assess the functionality of HLA-universal PLTs in vivo, $1 \times 10^{6} \mathrm{HLA}$-silenced MKs were infused into NOD/SCID/IL-2R $\gamma \mathrm{c}^{-/}$ mice with or without anti-HLA antibodies. PLT generation was evaluated by flow cytometry using anti-CD42a and CD61 antibodies.

Results: HLA-universal PLTs demonstrated to be functionally similar to blood-derived PLTs. Lymphocytotoxicity assays showed that HLA-silencing efficiently protects MKs against HLA antibody-mediated complement-dependent cytotoxicity. 80-90\% of HLA-expressing MKs, but only $3 \%$ of HLA-silenced MKs were lysed. In vivo, both HLA-expressing and HLA-silenced MKs showed human PLT production (up to $0.5 \%$ within the PLT population) when anti-HLA antibodies were absent. However, in presence of anti-HLA antibodies HLA-expressing MKs were rapidly cleared from the circulation of mice, while HLA-silenced MKs escaped HLA antibody-mediated cytotoxicity and human PLT production was detectable up to 11 days.

Conclusion: Our data show that HLA-silenced PLTs are functional and efficiently protected against HLA antibody-mediated cytotoxicity. Provision of HLA-universal PLT units may become an important component in the management of patients with PLT transfusion refractoriness.

\section{ZTH-V04 \\ Culture in human serum diminishes the adhesion of human adipose stromal cells to laminin and delayes integrin signalling}

\section{${ }^{\star}$ K. Bieback', S. Elvers-Hornung ${ }^{1}$, I. Brinkmann', T. Gloe ${ }^{2}$ H. Klüter ${ }^{1,2}$ \\ ${ }^{1}$ Institut für Transfusionsmedizin und Immunologie, Stammzell-Labor, Mannheim, Germany \\ ${ }^{2}$ Medical Faculty Mannheim, Cardiovascular Physiology, Mannheim, Germany}

Background: The clinical application of mesenchymal stromal cells (MSC) requires a production process compliant with Good Manufacturing Practice (GMP) guidelines. Pooled blood group AB human serum (HS) has been used to replace fetal bovine serum (FBS), critically rated by the regulatory agencies, supporting the expansion of adipose tissue-derived mesenchymal stromal cells (ASC). Comparing HS- and FBS-ASC revealed differentially expressed adhesion and extracellular matrix-associated molecules raising the question whether the serum source differentially affects adhesion of ASC.

Methods: Because previous microarray studies revealed a significant reduced expression of integrin a6 (CD49f) in HS-ASC, we compared ASC cultivated in FBS and HS with respect to expression of integrin subsets forming the laminin receptor as well as integrin signalling upon adhesion to laminin.

Results: Consistent with a lower CD49f expression intensity of HS-ASC, adhesion to laminin was significantly reduced compared to FBS-ASC. Blockage of CD29 (integrin a6) and CD49f, but not of CD104 (integrin b4), reduced the adhesion to laminin, pointing at integrin a6/31 (VLA-6) as functional laminin receptor on ASC. Upon adhesion to laminin, very rapid changes of the cell shape occurred, characterized by lammelipodia formation. Downstream signal transducers of integrin, $p$-FAK and p-PAX were expressed predominantly at these focal adhesion sites and indicated a delayed integrin signalling of HS-ASC compared to FBS-ASC.

Conclusion: Our data indicate that varying the serum supplement may alter clinical application-relevant characteristics of ASC like adhesion to extracellular matrix molecules. The reduced interaction with i.e. laminin may cause reduced homing and engraftment. Thus because major changes in the manufacturing process such as changing the source of supplement from FBS to HS can have a major impact on cellular functions the effects should be elucidated carefully. 
ZTH-V05

Identification of Interferon-alpha high and low expressing human plasmacytoid dendritic cell subsets exhibiting distinct functional characteristics

\section{${ }^{*}$ S. Sapski, L. Schneider, G. Michel, G. Bein, N. Baal, H. Hackstein}

University of Gießen, Clinical Immunology and Transfusion Medicine Gießen, Germany

Background: Human plasmacytoid dendritic cells (pDC) are rare antigen-presenting cells that are defined as principle Interferon-alpha (IFN- $\alpha$ ) producing cells and are currently exploited as potential cell therapeutics in antiviral and anticancer therapy strategies. Currently it is believed, that all human pDC express comparable high IFN- $\alpha$ levels after Toll-like receptor (TLR) triggering. By performing high-purity fluorescent activated cells sorting in combination with surface IFN- $\alpha$ staining on human pDC we show here that human pDC can be dissected in at least two subsets based on initial IFN- $\alpha$ expression exhibiting distinct functions.

Methods: IFN- $\alpha$ producing human $\mathrm{pDC}$ precursors were identified in peripheral blood samples from healthy blood donors with fluorochrome-labelled mAbs (BDCA- $4^{+}, \mathrm{CD} 123^{+}$, lineage cocktail-) in combination with surface IFN- $\alpha$ staining after TLR9 stimulation. IFN- $\alpha$ high and low producing pDC subsets were analysed for functional relevant surface markers (TRAIL, CD86) or separated by cell sorting and characterized with respect to cytokine producing capacity.

Results: IFN- $\alpha^{\text {low }}$ pDC exhibited significant faster upregulation of costimulatory CD86 surface expression in comparison to IFN- $\alpha^{\text {high }} \mathrm{pDC}$ from the same subjects. In contrast, IFN- $\alpha^{\text {high }} \mathrm{pDC}$ showed significant higher expression of cytotoxic CD253 (TRAIL) expression suggesting different killing activity of $\mathrm{pDC}$ IFN- $\alpha$ subsets $(18.4 \%$ TRAIL positive versus $7.6 \%$; p\&lt; 0.01$)$. Moreover, analysis of cytokine producing capacity of IFN- $\alpha$ pDC subsets revealed significantly higher TNF- $\alpha$ (p\&lt;0.01) and significantly lower IL- 8 producing capacity of IFN- $\alpha^{\text {low }} \mathrm{pDC}$ after TLR9 restimulation. Importantly, TLR restimulation of sorted IFN- $\alpha^{\text {high }}$ and IFN- $\alpha^{\text {low }}$ pDC subsets demonstrated that both subsets have comparable capacity to produce large IFN- $\alpha$ quantities but differ significantly in the initial response to TLR stimulation.

Conclusion: These data indicate that human pDC can be separated in at least two functional distinct subsets based on initial IFN- $\alpha$ expression after TLR9-dependent activation. IFN- $\alpha^{\text {high }}$ TRAIL ${ }^{\text {high }} \mathrm{pDC}$ staining identifies a novel pDC subset optimally equipped for tumoricidal activity.

\section{ZTH-V06}

Impact of cytokine activation on the expression of natural killer cell receptors and the lysis potential of natural killer cells

\section{${ }^{*}$ M.- A. Schlegel, S. Matthias, M. Peipp, C. Kellner, M. Gramatzki,} A. Humpe

II. Medizinische Klinik, Sektion für Stammzell- und Immuntherapie, Haus 43, Kiel, Germany

Background: Natural cytotoxicity of NK cells is balanced by inhibitory and activating signals. Activation of NK cells occurs by interaction of natural cytotoxicity receptors (NCR) and their corresponding ligands expressed on distressed cells. NK cells are inhibited by interplay of inhibitory killer cell immunoglobulin-like receptors (KIR) and corresponding HLA class I molecules on cells displaying a "self" phenotype. Here, the impact of short term NK cell pre-activation by interleukin 2 (IL-2) or IL-15 on the expression of NCR and KIR as well as NK cell cytotoxicity was analyzed.

Methods: NK cells were obtained by negative selection of leukocytes from healthy donors using magnetic activated cell sorting (MACS). After overnight activation with IL-2 (100U/ml) or IL-15 (10ng/ml), NK cell cytotoxicity was measured in ${ }^{51} \mathrm{Cr}$-release assays using different tumour cell lines as target cells. KIR type of each donor was determined by RT-PCR.
Expression levels of KIR and NCR were quantified by flow cytometry. For further analysis, only NK cells from donors displaying a significant lysis of NK-sensitive K562 cells ( $\geq 5 \%$, effector-to-target (E:T) ratio: 5:1) were considered.

Results: Compared to untreated NK cells the lysis of K562 cells increased significantly after pre-activation of NK cells with IL-2 (median lysis $20 \%$ vs. $32 \%$ ) or IL-15 (median lysis $45 \%$ ). Equivalent findings were observed for the HLA class I positive myeloma cell line L363 (median lysis untreated: $12 \%$, IL-2: 22\%, IL-15: $34 \%$ ). Relating to the lysis of L363, donors were classified into responder $(\geq 10 \%$ lysis, E:T ratio 5:1, $\mathrm{n}=11)$ and non-responder $(\mathrm{n}=10)$. As expected, NK cells of a significant higher number of responders were negative for KIR2DL2, an inhibitory KIR recognizing HLA molecules of the HLA-C1 supertype $(\mathrm{P}<0.02)$ expressed by L363 cells (HLA Cw3, Cw7). The surface expression levels of KIR and NCR were slightly increased on NK cells treated with IL-2 or IL-15, but KIR types were not affected by short term pre-activation.

Conclusion: As short term IL-2 / IL-15 treatment enhances the natural cytotoxicity of NK cells without affecting inhibitory KIR phenotype, pre-activated NK cells of KIR-HLA mismatched donors may be a powerful NK cell source to eliminate residual tumour cells in patients. To further enhance the lytic properties of pre-activated NK cells and in particular to elevate the selectivity of tumour cell targeting in vivo, a combination of pre-activated NK cells with monoclonal antibodies specific for appropriate target antigens on tumour cells might be a promising approach.

\section{ZTH-V07}

KIR2DL1-, KIR2DL2/3- and KIR3DL1- Receptors are Differentially Involved in Regulation of the NK Cell Activity

${ }^{*}$ R. Richter, P.S.A. Becker, S. Dombos, J.F. Hennecke, E. Seifried, C. Seidl

Institute of Transfusion Medicine and Immune Hematology, Blood Donation Service of the German Red Cross, Dept. Transplantation Immunology and Immunogenetics, Frankfurt, Germany

Background: Natural killer (NK) cells reveal the capacity to eliminate tumour and virally infected cells, and are of significance for the success of hematopoietic stem cell transplantation (HSCT). A number of studies suggest that especially inhibitory Killer Immunoglobuline like Receptors (iKIR) are of significance for the regulation of NK cell activity and for the success of HSCT. In former studies we confirmed that especially donor KIR2DL - recipient HLA-C ligand mismatch improves the clinical outcome in SCT.

Methods: To further evaluate the significance of KIR receptors for the regulation of NK cell activity we performed in vitro cytotoxicity assays with primary human NK cells from 12 volunteer healthy donors tested against the 721.221 cell line and primary AML blasts. The NK cells used were licensed for HLA-CGr1, HLA-CGr2, and HLA-Bw4 ligands.

Results: First, we found a high variance of the NK cells alloreactivity. 721.221 cells induced degranulation of NK cells from minimum $0.3 \%$ to maximum $49.7 \%$. Functional activity of NK cells incubated with primary AML blasts varied from $0 \%$ to $8.3 \%$. Second, KIR2DL1+, $\mathrm{KIR} 2 \mathrm{DL} 2 / 3^{+}$, and KIR3DL1 ${ }^{+} \mathrm{NK}$ cells reacted differently according to the missing-ligand mechanism. Compared to KIR2DL1 ${ }^{+}$and KIR3DL1 ${ }^{+}$ $\mathrm{NK}$ cells, KIR2DL2/3 $3^{+} \mathrm{NK}$ cells revealed a significantly reduced alloreactivity against 721,221 cells both in the absence of HLA-C group1 or HLA-C group1 and group2 ligands. In the experiments with AML blasts, $\mathrm{KIR} 3 \mathrm{DL} 1^{+} \mathrm{NK}$ cells revealed a significantly reduced alloreactivity compared to KIR2DL1 $1^{+} \mathrm{NK}$ cells. Furthermore, our results show that in addition to KIR- HLA ligand interactions further HLA dependent mechanisms are of relevance for the regulation of NK cell activity.

Conclusion: In conclusion, our results point to a differential regulation of the NK cell activity by KIR2DL1, KIR2DL2/3 and KIR3DL1 receptors and to additional HLA dependent, NK cell regulatory mechanisms. 


\section{ZTH-V08}

Overall survival after donor lymphocyte infusion for relapsed haematological malignancies after allogeneic haematopoietic cell transplantation

${ }^{*}$ N. Worel', P. Kalhs' ${ }^{2}$ W. Rabitsch², M. Mitterbauer'2, Z. Kuzmina ${ }^{2}$, G. Leitner', H.T. Greinix ${ }^{2}$

${ }^{1}$ Medizinische Universität Wien, Univ. Klinik für Blutgruppenserologie und Transfusionsmedizin, Wien, Austria

${ }^{2}$ Medizinische Universität Wien, Innere Medizin I,

Knochenmarkstranplantation, Wien, Germany

Background: Relapse is the major cause of death after allogeneic haematopoietic cell transplantation (HCT) and can be treated with donor lymphocyte infusions (DLI). Initial low dose DLI followed by escalating DLI doses have been used to minimize the risk of GVHD in patients with relapsed CML. In this study we retrospectively analyzed 70 patients who received DLI after HSCT. The primary objective of this analysis was to determine the effect of the initial DLI CD3 + cell dose on overall survival (OS).

Methods: Patients underwent myeloablative $(\mathrm{n}=40)$ or reduced intensity conditioning $(\mathrm{n}=30)$ followed by allogeneic HCT from related $(\mathrm{n}=50)$ or unrelated $(n=20)$ donors. Median age was 41 years (range, $18-63), 59 \%$ were male. Transplants were given for treatment of CML $(n=18)$, AML $(\mathrm{n}=27)$, MDS $(\mathrm{n}=3)$, ALL $(\mathrm{n}=7), \operatorname{MM}(\mathrm{n}=7)$, lymphoma $(\mathrm{n}=7)$ and others $(\mathrm{n}=1)$. DLI were administered for treatment of relapse/progression, mixed chimerism, or prophylactically according to the protocol. The median time intervals from HCT to relapse/progression and HCT to DLI were 12 (range, 1-100) and 14 months (range, 1-169), retrospectively. Thirty-one patients received DLI with or without imatinib, whereas in 39 cytoreductive chemotherapy before DLI was administered. Initial DLI CD3+ cell dose/kg was $\leq 1 \times 10^{7}$ ( $\mathrm{n}=25$; Group A), \&gt; 1.0 to $\leq 10 \times 10^{7}$ ( $\mathrm{n}=25$; Group B), and \&gt; $10 \times 10^{7}(\mathrm{n}=17$; Group C), in 3 patients the cell dose was not available. Twenty patients received escalating doses of CD3+ cells.

Results: Twenty-six (37\%) patients are alive after DLI with a median overall survival (OS) of 16 (range, 0.6-210) months. OS at 2 years for Group A, B and C were $52 \%, 56 \%$ and $33 \%$, respectively (Figure 1) Initial DLI CD3+ cell dose \&gt;10x107/kg was statistically significant associated with an increased risk of overall mortality compared to lower doses ( $\mathrm{p}=0.038)$. Forty-five (63\%) patients responded to DLI, 21 of them relapsed after initial response, and 20 did not respond, respectively. Three patients showed stable disease, and in 2 patients response evaluation is pending. Higher CD3+ DLI doses did not result in lower relapse rates or better response. Sixteen (23\%) patients developed acute or chronic GVHD after DLI. The risk for GVHD was significantly higher in patients receiving higher doses of $\mathrm{CD} 3+($ Group $\mathrm{B}$ and $\mathrm{C}$ ) compared to lower doses (Group A; $\mathrm{p}=0.033$ ). Five patients died due to severe GVHD.

Conclusion: Our results show that an initial DLI CD3 + cell dose $/ \mathrm{kg}$ \&gt; $10 \times 10^{7}$ is associated with increased risks of mortality after DLI, without reducing the risk of relapse. These findings are clinically relevant, and support a recommendation to infuse $\leq 10 \times 107 \mathrm{CD} 3$ cell $/ \mathrm{kg}$ for treatment of recurrent hematological malignancies after HCT. Additionally, molecular monitoring of patients is warranted to allow the use of DLI at an early relapse stage.

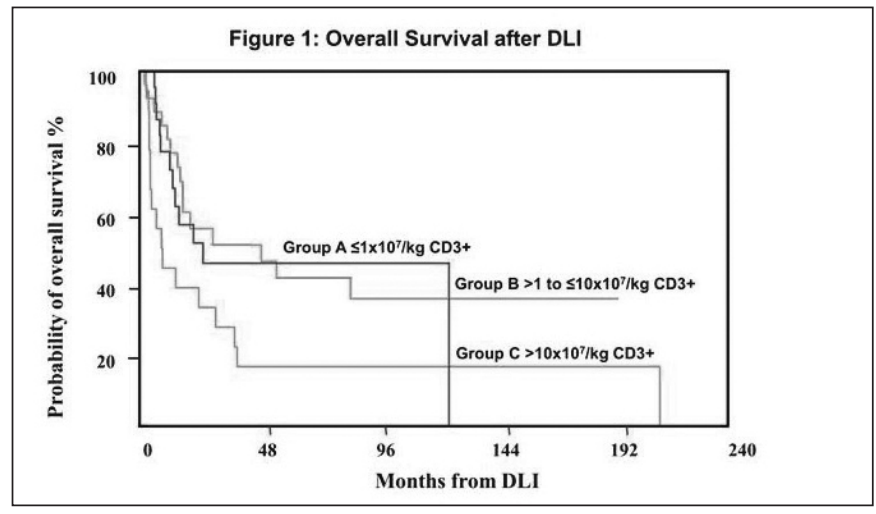

ZTH-V09

\section{Comparison of the content of regulatory} CD3+CD4+CD25+ T-cells in Umbilical Cord Blood (UCB) compared to leukapheresis products collected from adult blood donors

\author{
${ }^{*}$ E. Strasser ${ }^{1}$, M. Caemmerer ${ }^{1}$, W. Bochskanl ${ }^{1}$, J. Strobel', \\ T. Goecke², R. Eckstein ${ }^{1}$ \\ ${ }^{1}$ Universitätsklinikum Erlangen, Transfusionsmedizinische und \\ Hämostaseologische Abteilung, Erlangen, Germany \\ ${ }^{2}$ Frauenklinik des Universitätsklinikums Aachen, Pränatale Medizin und \\ Spezielle Geburtshilfe, Aachen, Germany
}

Background: Umbilical Cord Blood (UCB) units are of increasing interest for stem cell transplantation. Multiple-unit pooled UCB was recently reported as a valuable source of therapeutic regulatory T cells (Treg). (1) Herein, it was suspected that UCB product yields are far fewer than in adult peripheral blood. Thus, a comparison between UCB and leukapheresis products (LP) has been performed.

Ref.: 1. Milward K, Issa F, Hester J, et al. Multiple Unit Pooled Umbilical Cord Blood is a Viable Source of Therapeutic Regulatory T Cells. Transplantation 2013; 95:85-93.

Methods: T cell subset counts were compared between $67 \mathrm{UBC}$ units of neonates compared to leukapheresis products of 31 adult healthy leukocyte donors. Leukapheresis was performed on the COM.TEC cell separator (autoMNC program). $\mathrm{T}$ cell subpopulations (CD 3+, CD4+, CD25+ and $\mathrm{CD}$ 127- T cells) were analyzed by flow cytometry (FACS Calibur, BD, USA). The statistical analysis was calculated with SPSS (version 19.0).

Results: In UCB of neonates, the mean concentration of leukocyte (WBC) subsets was as follows (male vs. female): lymphocytes (3448/ $\mu 1$ vs. $3860 / \mu 1), C D 3+T$ cells $(2180 / \mu 1$ vs. $2422 / \mu 1), C D 3+C D 4+T$ cells $(1470 / \mu 1$ vs. $1660 / \mu 1)$, subset of CD25+ T cells $(237 / \mu 1$ vs. $276 / \mu 1)$ and Tregs $(101 / \mu 1$ vs. $107 / \mu 1)$, which yielded in $7.98 \pm 3.16 \times 10 \mathrm{e} 6$ Tregs in male and $7.80 \pm 4.50 \times 10 \mathrm{e} 6$ Tregs in female neonates in UCB harvests, respectively. The mean age of healthy blood donors ( 26 male, 5 female) was 31.9 years (range: 19 - 58 years). In relation to UCB units, LA showed significantly lower lymphocyte concentration $(p<0.001)$. Except for the concentration of CD25+ T-cell subset, which was significantly higher in LA, all other T cell subsets including Tregs were significantly lower in LA compared to UCB (see Table 1).

Conclusion: In contrast to the recent observation on the content of Tregs in UCB compared to adults, reported by Milward et al. (University of Oxford), our results suggest a significantly higher concentration of Tregs in UCB compared to LA of adult healthy blood donors. According to the collection of Tregs for clinical application, high volume UCB units may be sufficient and UCB pooling for a "Multiple Unit Pooled UCB" may be avoided. The strategy whether to pool UCB has to be proven in every single case depending on the yield of Tregs needed for the required therapeutic dose.

Table 1:
\begin{tabular}{|l|l|l|l|}
\hline Parameter $($ mean \pm SD $)$ & UCB $(n=67)$ & LP $(n=31)$ & -value \\
\hline CD3+ Tcells $($ per $\mu \mathrm{l})$ & $2300 \pm 661$ & $1156 \pm 329$ & $<0.001$ \\
\hline CD3+CD4+ cells $($ per $\mu \mathrm{l})$ & $1568 \pm 500$ & $623 \pm 257$ & $<0.001$ \\
\hline CD25+ Tcells $($ per $\mu \mathrm{l})$ & $256 \pm 94$ & $409 \pm 201$ & $<0.001$ \\
\hline CD25+CD127- Treg (per $\mu \mathrm{l})$ & $104 \pm 36$ & $52 \pm 26$ & $<0.001$ \\
\hline p-value (U-Test) &
\end{tabular}


ZTH-V10

\section{G-CSF impairs the antiviral immune response of T cells in stem cell donors: Implications for the selection of T-cell donors}

${ }^{*}$ C. Bunse ${ }^{1}$, S. Borchers ${ }^{2}$, P. Varanasi ${ }^{2}$, S. Tischer ${ }^{1}$, C. Figueiredo ${ }^{1}$, L. Goudeva' ${ }^{1}$ U. Kalinke ${ }^{3}$, B. Maecker-Kolhoff', U. Koeh/ ${ }^{5}$, A. Ganser ${ }^{2}$, E. Mischak-Weissinger ${ }^{2}$, R. Blasczyk ${ }^{1,2}$, ${ }^{*}$ B. Eiz-Vesper ${ }^{1}$

${ }^{1}$ Medizinische Hochschule Hannover, Institut für Transfusionsmedizin, Hannover, Germany

${ }^{2}$ Medizinische Hochschule Hannover, Abteilung Hämatologie,

Hämostaseologie, Onkologie, Hannover, Germany

${ }^{3}$ Twincore, Hannover, Germany

${ }^{4}$ Medizinische Hochschule Hannover, Pädiatrische Hämatologie und Onkologie, Hannover, Germany

${ }^{5}$ Medizinische Hochschule Hannover, Institut für zelluläre Therapien Hannover, Germany

Background: Adoptive transfer of antiviral T cells has become a powerful and promising tool to complement impaired immune reconstitution in patients after allogeneic hematopoietic stem cell transplantation (HSCT) HSCT patients are at risk of experiencing recurrent reactivation of persisting viruses like CMV and EBV or lytic viruses like ADV. If available, seropositive stem cell donors may serve as a source for antiviral T cells Recently, expansion of cytotoxic T-lymphocytes (CTL) from G-CSF mobilized stem cell grafts has been suggested as a source for antiviral CTL. Methods: There is, however, growing evidence that G-CSF mobilization has negative effects on T-cell function. Here, we analysed the effects of G-CSF on antiviral T cells in vitro and in vivo in 87 mobilized stem cell donors and 25 unmobilized controls. The number of CMV-, EBV- and ADV-CTL were assessed using HLA-matched virus-specific multimers (A*01:01, A*02:01, A*24:02, B*07:02, B*08:01, B*35:01). Functional activity was tested by IFN- $\gamma$ ELISPOT assay after stimulation with overlapping peptide pools (CMV: pp65, IE-1; EBV: EBNA1, LMP2A, BZLF1; ADV5: hexon).

Results: Interestingly, the absolute number of antiviral CTL detected after G-CSF stimulation and compared to unmobilized samples was not influenced by G-CSF, but function of T cells in response to all viral antigens was significantly impaired as expressed by a mean reduction in IFN- $\gamma$ secretion of $73.9 \%$ (CMVpp65 80.4\%, CMVIE-1 79.07\%, EBVEBNA1 76.6\%, EBVLMP2A 57.75\%, EBVBZLF1 75.8\%). To underline these results in vitro stimulation assays were performed in presence or absence of G-CSF using single HLA-restricted peptides. Expansion rates for G-CSF-cultured T cells did not differ from untreated cells (e.g. A*02:01_CMVpp65: 2.6 vs 3.0 fold increase, B*08:01_EBVBZLF1: 20.9 vs $\overline{20.3}$ fold increase), but IFN- $\gamma$ secretion was reduced by $39.5 \%$ (mean all tested peptides).

Conclusion: Our study shows that during G-CSF mobilization, the functional activity of antiviral memory $T$ cells is impaired, indicating that even stem cell donors may not be the best source of T cells. Taken together, we suggest that (1) CTL for adoptive transfer in patients at high risk for viral reactivation (e.g. haploidentical T-cell depleted HSCT) should be harvested prior to G-CSF mobilization in order to avoid an adoptive transfer of functionally impaired antiviral CTL and (2) third party donors should be considered in patients at need of antiviral CTL early after HSCT.
ZTH-V11

\section{Phenotypic and morphologic characterization of dendritic cells in a patient suffering from blastic plasmacytoid dendritic cell neoplasm (BPDCN)}

${ }^{\star}$ C. Grabowski ${ }^{1}$, P. Hoyer ${ }^{1}$, U. Lippert ${ }^{2}$, M. Brunner ${ }^{2}$, U. Krause ${ }^{3}$, A. Florschütz ${ }^{4}$, M. Plauth ${ }^{4}$, H. Kroll ${ }^{1}$

${ }^{1}$ Red Cross Blood Transfusion Service NSTOB, Institute for Transfusion Medicine Dessau, Dessau, Germany

2Städtisches Klinikum Dessau, Department of Dermatology, Venerology, Allergology and Immunology, Dessau, Germany

${ }^{3}$ Städtisches Klinikum Dessau, Institute of Pathology, Dessau, Germany ${ }^{4}$ Städtisches Klinikum Dessau, Department of Internal Medicine, Haematology and Oncology, Dessau, Germany

Background: Dendritic cells (DCs) play a central role in regulatory processes of the immune system by producing cytokines, processing and presenting self and foreign antigens to T- cells. They participate in antiviral and antitumor responses and are used for immunotherapy of malignancies and viral infectious diseases. Different types of DCs have been characterized by specific cell surface markers. Here we describe a case of a rare malignancy of DCs.

Case Report: The 79-year-old male patient was admitted to hospital because of multiple rapid progressive indolent, maculous, papulous, redish-brown coloured cutaneous lesions at the head, trunk and arms. Furthermore he showed gum bleeding and reported on night sweats. Laboratory investigations indicated thrombocytopenia $(72.000 / \mu \mathrm{l})$, mild anemia $(12,9 \mathrm{~g} / \mathrm{dl})$ and normal differential blood count. Abdomen sonography only showed a slightly enlarged spleen $(\varnothing 13 \mathrm{~cm})$. Due to thrombocytopenia investigation of bone marrow (BM) was performed.

Methods: To characterize the phenotype of WBCs in BM aspirate immunofluorescence stained mAbs against lineage markers were used. Cells were analyzed by eight-colour multiparametric flow cytometry. Additionally, cytomorphology, immunohistochemistry and cytogenetic analysis of $\mathrm{BM}$ and skin biopsy were evaluated.

Results: Scatterplot analysis of BM cells showed a large population $(17 \%)$ of agranular cells with weak expression of CD45. These cells were strongly positive for CD56, positive for CD4, CD123, CD38, CD36 $(30 \%)$ and negative for progenitor, myeloid and lymphoid cell lineage specific markers as CD34, CD117, MPO, TdT, cyCD3, sCD3, CD2, CD7, CD8, CD57, TCR $\alpha \beta / \gamma \delta$, CD1a, CD79a, CD19, and CD20. Immunohistochemistry of BM and skin biopsy revealed the presence of nodular, diffuse infiltration of monomorphous cells with a blastoid morphology and expression of both CD56 and CD4. Cytomorphology of BM showed hyperplasia and infiltration by ambiguous undifferentiated blastoid cells with fine chromatin and one to four nucleoli replacing genuine hematopoesis. In cytogenetic analysis (karyotype, $B C R-A B L$, MLL rearrangement) no abnormalities were found.

Conclusion: We describe a rare case of DC malignancy, BPDCN, formerly known as CD4+CD56+ hematodermic neoplasia. Since BPDCN is characterized by rapid progression, high relapse rate despite chemotherapy and poor prognosis, precise surface antigen profiling is mandatory. Our extended panel of cell surface markers allowed the rapid flow cytometric diagnosis and will further reveal a tool for studies of development and function of DCs. 


\section{ZTH-V12}

\section{Validation study on human adipose stromal cells manufacturing for cell therapy}

${ }^{*}$ M. Karagianni', H. Klüter', S. Wigand², H.-G. Machens'2, N. Papadopulos' ${ }^{2}$ K. Bieback'

${ }^{1}$ Institut für Transfusionsmedizin und Immunologie, Medizinische Fakultät Mannheim, Universität Heidelberg; DRK-Blutspendedienst Baden-

Württemberg - Hessen gGmbH, Mannheim, Germany

${ }^{2}$ Klinikum rechts der Isar, Klinik und Poliklinik für Plastische Chirurgie und Handchirurgie, Technische Universität München, München, Germany

Background: Adipose-derived stromal cells are interesting candidates for cell therapy due to their multilineage differentiation potential, their immunomodulatory properties and proregenerative features. Adipose stromal cells (ASC) can be isolated in a high cell frequency from adipose tissue by low risk liposuction. We studied different manufacturing modalities to establish a GMP protocol for ASC manufacturing.

Methods: 86 adipose tissue (AT) preparations were obtained from 29 donors as resections or lipoaspirates from different anatomical sites. Lipoaspirates were processed under GMP conditions to isolate the stromal vascular fraction (SVF) containing the ASC. Sterility testing was performed at different manufacturing steps. SVF were analysed according to cell yield / $\mathrm{ml}$ AT, cell vitality, precursor frequency by CFU-F assay, immune phenotype and expanded to derive mesenchymal stromal cells (MSC). MSC were then characterised by flow cytometry and differentiation towards the adipogenic and osteogenic lineage. Statistical analysis was performed regarding amongst others age, gender, tissue localisation, body mass index (BMI) and sampling system.

Results: A mean of $8,4 \times 10 \mathrm{E} 4$ vital cells / $\mathrm{ml} \mathrm{AT}$ and $384 \mathrm{CFU} / \mathrm{ml}$ AT was obtained. Interestingly there was a significant positive impact of the donor age and BMI on the ASC yield and cell vitality. However, no further significant differences were detected regarding e.g. anatomic localization, the sampling system on ASC yield and MSC characteristics. In the sterility testing $75 \%$ of the lipoaspirate were negative; in $25 \%$ bacterial contamination mainly with skin flora was detected in the unprocessed lipoaspirate.

Conclusion: Via liposuction with sterile systems and isolation under GMP conditions, ASC can be easily obtained and applied in an adequate cell dose in regenerative settings without cell expansion in vitro. Donor age and BMI impacts the cell yield and vitality of ASC in an accordant manner in contrast to stromal cells of bone marrow, which decrease with age. Thus in contrast to bone marrow, adipose tissue is a feasible, autologous cell source in a broader spectrum of patients.

\section{ZTH-V13}

\section{Effect of high dose irradiation on human bone marrow- derived MSC}

\section{N. Fekete ${ }^{1}$, A. Erle ${ }^{2}$, G. Schmidtke-Schrezemeier ${ }^{1}$, D. Fürst ${ }^{1}$,} M. Rojewski ${ }^{2},{ }^{*} H$. Schrezenmeier ${ }^{1}$

${ }^{1}$ IKT Ulm, DRK-Butspendedienst Baden-Württemberg - Hessen, Ulm, Germany ${ }^{2}$ Universität UIm, Institut für Transfusionsmedizin, Ulm, Germany

Background: Cell therapy using MSC is of high interest in various indications. As the pleiotropic effects mediated by MSC rely mostly on their unique secretory profile, long-term persistence of ex vivo expanded cells in the recipient may not always be desirable. Irradiation is a routine procedure in transfusion medicine to prevent long-term persistence of nucleated cells and could therefore be applied to MSC.

Methods: We have exposed human bone marrow-derived MSC to 30 or 60 Gy of $\gamma$-radiation and assessed cell proliferation, clonogenicity, differentiation capacity, cytokine levels in media supernatants, and surface receptor expression profiles. mRNA expression of proto-oncogenes / cell cycle regulators (TERT, TERC, P53, CDKN1A, CDKN2A, MYC), stemness markers (NANOG, KLF4), as well as DNA damage markers (ATM, ATR, BRCA1, CHEK1, CHEK2, MDC1, TP53BP1) were measured by qPCR. Additionally, genetic stability was analysed by karyotyping.
Results: Irradiated MSC show a significant decrease in proliferation and CFU-F. A subpopulation of surviving cells remains able to differentiate and form colonies even 28 days after irradiation. Irradiated MSC showed stable expression of CD73, CD90 and absence of CD3, CD34, CD45 during the 16 week follow-up period. Levels of VEGF were increased and those of PDGF-AA, PDGF-AB/BB were decreased in culture media of non-irradiated cells; irradiated MSC showed an inverse pattern. IL-6 levels increased regardless of irradiation. Radiation-resistant cells showed positive $\beta$-galactosidase activity 14 days after irradiation. mRNA levels of CDKN1A and CDKN2A increased, levels of TERC decreased. Stemness marker KLF4 showed decreased expression and the profile of DNA damage markers was altered.

Conclusion: In conclusion, a subgroup of MSC showed resistance to high doses of radiation. The surviving cells retained their tri-lineage differentiation capacity, surface marker profile and ability to form colonies, but became prematurely senescent. mRNA expression levels of cell-cycle and stemness markers indicated a cell cycle arrest and also premature cellular senescence after exposure to high doses of $\gamma$-radiation.

\section{ZTH-V14 \\ Co-cultures of cardiomyocytes and bone marrow mesenchymal stromal cells: Modelling myocardial msc integration}

\section{A. Erle ${ }^{1,2},{ }^{*}$ R. Lotfi', ${ }^{1,}$ A. Liebold ${ }^{3}$, M. Hönicka ${ }^{3}$, D.C. Tradowsky ${ }^{1,2}$} H. Schrezenmeier ${ }^{1,2}$

${ }^{1}$ German Red Cross Blood Transfusion Service Baden-Württemberg Hessen, Institute of Clinical Transfusion Medicine and Immunogenetics, Ulm, Germany

2University of Ulm, Institute of Transfusion Medicine, Ulm, Germany

${ }^{3}$ Ulm University Medical Center, Department of Cardiothoracic and Vascular Surgery, UIm, Germany

Background: Stem cell therapy of myocardial infarction may have a twofold effect: paracrine interactions between stem cells and cardiomyocytes as well as stem cell transdifferentiation/cell fusions. This study investigated direct cell-cell interactions in a co-culture model.

Methods: Murine or human bone marrow mesenchymal stromal cells (BM-MSCs) were co-cultured with murine or human cardiomyocytes at different seeding ratios ranging from 1:5 to 1:100. For cell tracking purposes human cardiomyocytes and murine BM-MSCs constitutively expressed RFP and GFP, respectively. Cultures were analyzed by a qPCR panel consisting of 14 cardiomyocyte markers, and co-cultures were assessed by video fluorescence microscopy.

Results: Native BM-MSCs were found to already express three of fourteen tested cardiomyocyte markers: FGF2, GJA1 and MEF2C. Co-cultures remained viable for at least four weeks. Cardiomyocytes showed spontaneous beating which was most prominent at seeding ratios of 1:10 (BM-MSC : cardiomyocyte). Video microscopy revealed a few cells of BM-MSC origin which appeared to be beating, and several fused cells from both cell sources.

Conclusion: Co-cultures demonstrated that cardiomyocytes and BMMSCs may coexist for extended periods of time, thus allowing mutual interactions. Depending on relative cell numbers, beating of cardiomyocytes was not hindered by BM-MSCs. Both apparently beating cells of BM-MSC origin as well as fused cells may indicate regenerative effects, albeit at low frequencies. Methods currently under development will allow isolating the cell types after co-culture and analyzing them separately to gain further insight into transdifferentiation. 


\section{ZTH-V15}

\section{Anti-HLA antibody-dependent induction of vascular cell adhesion molecule-1 expression is modulated by heme oxygenase-1 in human endothelial cells}

${ }^{*}$ E. Zilian', H. Saragih', J. Larmann², P. Demmer', C. Figueiredo', B. Eiz-Vesper', R. Blasczyk', J. Becker'², G. Theilmeier ${ }^{2}$,

\section{S. Immenschuh}

${ }^{1}$ Medizinische Hochschule Hannover, Institut für Transfusionsmedizin, Hannover, Germany

${ }^{2}$ Medizinische Hochschule Hannover, Hannover, Germany

Background: Transplant vasculopathy (TV) is a key limiting factor for long-term graft survival after kidney and heart transplantation and is characterized by activation and proliferation of endothelial cells (ECs) in allograft vessels. Interactions of donor-specific antibodies (abs) against human leucocyte antigens (HLAs) with ECs play a major role in the pathogenesis of $\mathrm{TV}$, but the underlying regulatory mechanisms are not well understood.

Methods: To examine the effects of anti-HLA abs on ECs, cell cultures of human umbilical vein ECs (HUVECs), human aortic ECs (HAECs) and human dermal microvascular ECs (HDMVECs) were treated with the monoclonal anti-HLA class I ab w6/32 for different time periods. Expression of inducible pro-inflammatory molecules such as vascular cell adhesion molecule (VCAM)-1, intercellular adhesion molecule (ICAM)-1, interleukin-8 (IL-8) and MCP-1 were determined by real-time RT-PCR. To elucidate the underlying mechanisms of anti-HLA class I ab-dependent up-regulation of pro-inflammatory genes, pharmacological inhibitors for specific potential signaling pathways were applied. Moreover, to investigate the potential role of the anti-inflammatory endothelial enzyme heme oxygenase (HO)-1 in the regulation of pro-inflammatory genes, HO-1 was modulated by pharmacological compounds and by a small interfering (si)RNA knockdown approach.

Results: Binding of w6/32 to ECs markedly up-regulated gene expression of the pro-inflammatory adhesion molecule VCAM-1 in a time-dependent manner. Similarly, ICAM-1, IL-8 and MCP-1 were up-regulated by w6/32. This up-regulation was mediated via the phosphatidylinositol-3-kinase (PI3K)/Akt signaling cascade. Blocking of HO-1 activity by zinc-protoporphyrin (PPIX) and siRNA-mediated HO-1 knockdown enhanced VCAM-1 gene expression, whereas up-regulation of HO-1 with the HO-1 inducer cobalt-PPIX markedly inhibited w6/32-mediated VCAM-1 induction. Accordingly, w6/32 increased adhesion of THP-1 monocytes to ECs in an in vitro adhesion assay, which was counteracted by pharmacological up-regulation of $\mathrm{HO}-1$.

Conclusion: These findings suggest that the anti-HLA class I ab-dependent induction of pro-inflammatory adhesion molecules in human ECs is down-regulated by HO-1. Thus, endothelial HO-1 may serve as a therapeutic target in ab-mediated TV after solid organ transplantation

\section{Poster: Zelltherapie \& Gewebe}

\section{ZTH-P01}

A new approach for analysing avitality of tumor cell lysate used in dendritic cell vaccination trials

\section{Müller', D. Hartmann', C. Ecker', C. Keck', *H. Eichler'}

${ }^{1}$ Saarland University Hospital , Institute of Clinical Hemostaseology and Transfusion Medicine , Homburg/Saar, Germany

${ }^{2}$ University of Applied Sciences, FH, Applied pharmacy , Zweibrücken, Germany

Background: A prerequisite for the processing of clinically applicable cell preparations in dendritic cell (DC)-based immunotherapy is a fine-grained validation protocol. Loading immature DC with autologous tumor lysate (TL) is a crucial point with regard to safety aspects. For this purpose, the avitality of TL has to be clearly characterized after the reconditioning process. Trypan blue staining seems to be the commonly used method for exclusion of vital tumor cells, but it shows not to be optimal regarding sensitivity and should therefore be confirmed by further approaches.

Methods: Particle size, size distribution and morphology, i.e. shape of the TL particles, were assessed using a Morphologi G3 (Malvern Instruments). Analysis was performed by screening particle by particle. The approach was affirmed by taking particularly the cell size distribution into account: cell size of viable tumor cells was compared to the devitalised TL measured by CASY TT cell counter (Roche) for high resolution. In parallel, we determined ATP content as vital cell marker with luminescence based CellTiterGlo assay (Promega). A titration curve was measured with freshly isolated PBMCs alone or spiked with rising concentrations of TL. Results: Characterization of TL composition by Morphologi G3 clearly identified fragmented, non-cellular structures, thus proving the destruction of the cells. The size of the fragments was significantly lower than the size of the vital cells, i.e. fragments possessed a size range from 0 to $10 \mu \mathrm{m}$, whereas vital cells possessed a mean size of $20 \mu \mathrm{m}$. These results were confirmed by the cell distribution measure in high resolution record of the CASY TT counter. All blue cell-like particles identified by trypan blue staining did not show any viability determined by the ATP assay.

Conclusion: The tested methodology for the proof of devitalisation of tumor tissue extends the validity of the insensitive trypan blue staining: the ATP assay covers the functionality part, and measuring by G3 Morphology the structural part. The goal to assess the bioinactivity of TL in the validation process of DC preparation can clearly be reached by a combination of the tested methods. This approach showed to be convincing by offering a detailed impression of the constitution of TL used in DC vaccination therapy.

\section{ZTH-P02 \\ Evaluation of methods for counting and viability testing of monocytes and dendritic cells}

\section{H. Dorothee, I. Müller, C. Ecker, ${ }^{*} H$. Eichler}

Saarland University Hospital , Institute of Clinical Hemostaseology and Transfusion Medicine , Homburg/Saar, Germany

Background: Key steps in experimental workflows are counting of cells and determining of cell viability as an instrument of quality control. The widely used manual hemocytometer $(\mathrm{mH})$ is a mainstay of cell biology labs to determine cell counts; but this method is time consuming, shows lack of statistical robustness and result in variability between users. On the other hand, automated cell counters provide quick counting of cells and a live/dead ratio in a single step. Therefore, in a comparison study, five different methods for cell counting and assessment of viability were tested regarding reproducibility and precision.

Methods: Three PBMC samples from leukapharesis and three DC samples were analysed. In addition, a dilution series of one single PBMC sample was measured. Each of the samples were divided in final aliquots and measured in triplicate by the five methods: $\mathrm{mH}$ as reference method, automated cell counting devices (Sysmex KX-21N (Sysmex), CASY TTC150 (Roche), MoxiZ (Orflo)), and single platform flow cytometry analysis (FACS SPF) measuring the parameters CD14, CD45, CD11c, and 7-AAD (FACSCalibur, Becton Dickinson). The tested cell concentrations met the linear measurement range of each device. Percentages of viability and absolute counts of PBMCs, DCs, leukocytes and monocytes were analysed. Statistics were done by calculating the mean, the standard deviation, the coefficient of variation (CV) and the unpaired student's t-test

Results: Intra-laboratory reproducible results were obtained counting PBMCs and in dilution series by $\mathrm{mH}$, MoxiZ, Sysmex, CASY TTC-150, and FACS SPF. The most comparable results according to $\mathrm{mH}$ as reference method were obtained by FACS SPF with deviation from reference of only $16.74 \%$ for the three identical samples $(n=3)$. Determining the viability of PBMC samples, reproducible results were obtained by $\mathrm{mH}$, CASY TTC-150 and FACS SPF, but the most precise results regarded to $\mathrm{mH}$ were obtained by CASY TTC- 150 . 
Testing DC samples, reproducible results were obtained by Sysmex (CV range of 0.05 to 0.2 ), $\mathrm{mH}$ (CV range of 0.16 to 0.23 ), CasyTTC-150 (CV range of 0.10 to 0.12 )and FACS SPF (CV range of 0.09 to 0.23 ). Compared to $\mathrm{mH}$, none of the devices generated highly precise results. Determining the viability of DC, CASY TTC-150 and FACS SPF showed most reproducible results. Furthermore, FACS SPF offered comparable results in determining the $\mathrm{DC}$ viability

Conclusion: The comparison demonstrates that there isn't a universal cell counter for all subsets of cells. Therefore, different cell counters for different cellular samples have to be used to obtain valid results. FACS SPF and $\mathrm{mH}$ can be used to determine the cell count of a PBMC sample. For viability testing of PBMC samples, $\mathrm{mH}$ and CASY TTC-150 gave best results in our hands. In contrast to the other tested devices, FACS SPF provides measurements of cellular subtypes by antibody staining. This is a possibly explanation for the differences to $\mathrm{mH}$, because by $\mathrm{mH}$ it is not possible to differentiate the cell subtypes.

\section{ZTH-P03}

\section{Interleukin-21 induces regulatory $B$ cells in humans}

*S. Lindner', C. Kaltenmeier', T.F.E. Barth', C. Schuldhaus', T. Beyer ${ }^{1}$, P. Möller', H. Schrezenmeier ${ }^{1,3}$, B. Jahrsdörfer ${ }^{1,3}$

${ }^{1}$ Universität Ulm, Institut für Transfusionsmedizin, Ulm, Germany ${ }^{2}$ Universität Ulm, Institut für Pathologie, Ulm, Germany

${ }^{3}$ DRK Blutspendedienst Baden-Württemberg - Hessen, Ulm, Germany

Background: The pathogenic impact of tumor-infiltrating B cells is unresolved at present, however, some studies suggest that they may have immune regulatory potential. Here we report that the microenvironment of various solid tumors includes B cells that express granzyme B ( $\mathrm{GrB})$, and these $\mathrm{B}$ cells can be found adjacent to IL-21-secreting $\mathrm{CD} 4^{+} \mathrm{T}$ helper cells. Because $\mathrm{T}_{\text {regs }}$ and plasmacytoid dendritic cells are known to modulate $\mathrm{T}$ effector cells by a GrB-dependent mechanism, we hypothesized that a similar process may operate to modulate regulatory $\mathrm{B}$ cells $\left(\mathrm{B}_{\text {reg }}\right)$.

Results: IL-21 induced outgrowth of B cells expressing high levels of $\mathrm{GrB}$, which thereby limited T cell proliferation by a GrB-dependent degradation of the $\mathrm{T}$ cell receptor $\zeta$-chain. Mechanistic investigations into how IL-21 activated GrB expression in B cells to confer $B_{\text {reg }}$ function revealed a $\mathrm{CD} 19^{+} \mathrm{CD} 38^{+} \mathrm{CD} 1 \mathrm{~d}^{+} \mathrm{IgM}{ }^{+} \mathrm{CD} 147^{+}$expression signature, along with expression of additional key regulatory molecules including IL-10, CD25 and IDO. Notably, induction of GrB by IL-21 integrated signals mediated by surface IgM (BCR) and Toll-like receptors (TLR), each of which were enhanced with expression of the B cell marker CD5.

Conclusion: Our study is the first to show that IL-21 induces $\mathrm{GrB}^{+}$regulatory B cells in humans. They also establish the existence of human B cells with a regulatory phenotype in solid tumor infiltrates, where they may contribute to the suppression of anti-tumor immune responses. Together, these findings may stimulate novel diagnostic and cell therapeutic approaches to better manage human cancer as well as autoimmune and graft-versus-host pathologies.

\section{ZTH-P04}

\section{Assessment of increased CD4+CD25+FoxP3 T-regulatory cell ratio in acute respiratory distress syndrome}

*I. Rehfeld ${ }^{1}$, M. Adamzik ${ }^{2}$, C. Kreissig ${ }^{1}$, J. Peters ${ }^{2}$, J. Bux ${ }^{3}$ ${ }^{1}$ DRK-BSD West gGmbH, Zentralbereich Stammzelle, RatingenBreitscheid, Germany

${ }^{2}$ Universitätsklinikum Essen, Klinik für Anästhesiologie \& Intensivmedizin, Essen, Germany

${ }^{3}$ DRK-BSD West gGmbH, Hagen, Germany

Background: In the past, there have been numerous investigations studying the precise function of T-regulatory cells in human diseases. CD4+CD25+FoxP3+ T-regulatory lymphocytes cells (Tregs) efficiently migrate into inflammatory sites, and predominantly suppress peripheral inflammation. Up to now no data are available on the presence and function of Tregs in humans during acute respiratory distress syndrome (ARDS). With the hypothesis that Tregs decrease inflammatory process, we examined BAL and peripheral blood of 37 patients with ARDS and 8 control patients.

Methods: Within 3 hours of BAL we performed FACS analysis using FACS-Calibur flow cytometer (Becton Dickinson, San Jose, CA) and Treg Detection Kit human (Miltenyi Biotec, Bergisch Gladbach, Germany). This kit contains FITC-conjugated CD4 antibody and PE-conjugated CD25 antibody for surface staining and APC-conjugated FoxP3 antibody for intercellular staining.

First peripheral blood and BAL samples were incubated with CD4 FITC and $\mathrm{CD} 25 \mathrm{PE}$ antibodies for 10 minutes at $4^{\circ} \mathrm{C}$ than cells were fixed, permeabilized and stained with the anti-FoxP3-APC antibody for 30 minutes at $4^{\circ} \mathrm{C}$.

Gating was performed as follows: Autofluorescent debris was excluded using forward and side scatter. Gating of CD25 and FoxP3 positive cells was performed according to CD4 positive cell expression. Isotyp controls characterized the position of the quadrants. Dot plots show the percentage of CD25 and FoxP3- coexpressing CD4-postive cells.

Results: We were able to detect Tregs in BAL in patients suffering from ARDS as well as in control patients without ARDS. The mean ration of Tregs to all CD4- lymphocytes was 3 fold greater in ARDS non-survivors ( $16,5 \% ; \mathrm{P}=0,025)$ and almost 2 fold greater in ARDS survivors $(9 \%$ $; \mathrm{p}=0,015)$ compared to the control group (5.9\%). Multivariate Cox-regression analysis revealed the ratio of CD4+CD25+FoxP3+ T-regulatory lymphocytes in the BAL to be an important and independent prognostic factor for 30-day survival (HR 6,5; 95\% CI, 1,7-25; $\mathrm{p}=0,006$ ).

Tregs in blood samples did not show any differences between patients and controls.

Conclusion: In contrast to our hypothesis, that Treg cells can suppress inflammation process and therefore contribute to lung repair, increased T-regulatory cell ration in the admission BAL of patients with ARDS is an important and independent risk factor for 30-day mortality.

It seems, that very high levels of Treg cells led to suppressed function of T-effector cells via IL10

This was associated with increased disease severity and worse outcome.

\section{ZTH-P05 \\ Detection of clonal B cell populations using 454 sequencing}

\section{J. Weinberger ${ }^{1}$, J. Pröll', N. Niklas ${ }^{1}$, M. Danzer ${ }^{1}$, H. Polin ${ }^{1}$, J. Thalhamer ${ }^{2}$, ${ }^{*} \mathrm{C}$. Gabriel ${ }^{1}$}

${ }^{1}$ Red Cross Transfusion Service of Upper Austria, Linz, Austria 'University of Salzburg, Department of Molecular Biology, Salzburg, Austria

Background: In the present study we used 454 sequencing to analyze individuals' B cell receptor (BCR) heavy chains from blood samples by sequencing rearranged VDJ regions. The huge amount of sequences that are produced by next generation sequencing allows generation of patterns of an individual's B cell varieties at a specific time point, due to B cell variations being deducible from BCRs. Certain diseases like chronic lymphocytic leukemia (CLL) or post-transplant lymphoproliferative disorder (PTLD) show restrictions in terms of variation that can be used as a diagnostic marker.

Methods: DNA-extraction was done out of PBMCs from healthy donors, CLL patients and vaccinated (diphtheria toxoid + polio virus + tetanus toxoid) individuals. For amplification of rearranged IGH loci we used Biomed-2 primersets and multiplex PCR. From several possible sequencing strategies we decided to perform bidirectional amplicon sequencing using an unmodified 454 sequencing protocol on the Genome Sequencer FLX. The generated sequencing data were analyzed by IMGT/HighVQUEST followed by in-house developed bioinformatics tools for indepth visualization of VDJ and CDR3 clonality and variability.

Results: The results of variability calculations by analyzing CDR3 sequences and VDJ element combination elicited strong restrictions in the 
variation spectra of CLL patients, obviously triggered by overproliferating clones. Most abundant B cell clones in tested CLL blood samples represented $83-98 \%$ of all B cells compared to $0.2-1.3 \%$ in healthy controls. In addition, sensitivity and methodical limits of this approach were investigated. For that purpose, blood from vaccinated individuals was tested for immunization-induced restrictions or shifts in the VDJ rearrangement spectrum as well as the deviation in sequences gained by repeated sequencing of one sample. Both settings indicated that detecting minor changes poses a challenge which cannot be passed without extensive improvement of the method.

Conclusion: Analysis of B cell receptor patterns by using Roche 454 Life Sciences sequencing technology is an uncomplicated strategy to obtain an overview of B cell diversity and clonality and therefore a way to obtain very useful information on an individual's health. However the study indicated that detecting minor changes resulting from less pathologic infections and diseases poses a challenge that cannot be passed without further improvements of the method.

\section{ZTH-P06}

Rare cells in blood: Insulin like growth factor receptor I (IGF-IR) and vascular endothelial growth factor receptor 2 (VEGFR-2) are detectable by immunofluorescence on epithelial circulating tumor cells (e-CTCs) in patients with solid tumors

\section{${ }^{*}$ D. Zimon, M. Pizon, U. Pachmann, K. Pachmann}

Transfusionsmedizinisches Zentrum Bayreuth, Bayreuth, Germany

Background: Analysis of epithelial circulating tumor cells in the blood of cancer patients is a promising diagnostic field for estimating the risk for metastatic relapse and progression. The phenotypic characterization of e-CTCs may provide real-time information and can be of great value in therapy monitoring. IGF-IR and VEGFR-2 play a crucial role in tumor growth and the progression of cancer disease. Therefore the purpose of the current study was to investigate their expression on the e-CTCs.

Methods:

Methods

E-CTCs were determined from blood of 163 patients suffering from breast, prostate, colorectal and lung cancer. The number of vital e-CTCs and the expression of IGF-IR and VEGFR-2 were investigated using the maintrac ${ }^{\circledR}$ method.

Results: The expression of IGF-IR and VEGFR-2 on the surface of e-CTCs was detected in all types of cancer. The prevalence of e-CTCs with positive staining for IGF-IR and VEGFR-2 in patients with solid tumors is summed in the Table 1. The highest number of vital e-CTCs was observed in prostate cancer patients in comparison with other tumor types. A statistically high correlation was found between IGF-IR and VEGFR-2 expression on the e-CTCs in all cancer types. IGF-IR and VEGFR-2 expression on e-CTCs were positively associated with a higher number of e-CTCs only in breast cancer patients.

Conclusion: Our results demonstrated for the first time the expression of IGF-IR and VEGFR-2 on the e-CTCs in patients with breast, prostate, colorectal and lung cancer and thus constitute the basis for using antiIGF-IR and anti-angiogenic therapy for their elimination. With the main$\operatorname{trac}^{\circledR}$ approach we are able to precisely enumerate and phenotypic characterize e-CTCs, despite the fact that these cells occur rarely in the blood.
Table 1: The prevalence of e-CTCs with positive staining for IGF-IR and VEGFR-2 in patients with breast, prostate, colorectal and lung cancer

\begin{tabular}{|l|c|c|}
\hline Tumor types & $\begin{array}{l}\text { Number of patients with } \\
\text { positive staining for IGF-IR } \\
\text { on e-CTCs (\%) }\end{array}$ & $\begin{array}{l}\text { Number of patients with } \\
\text { positive ftaining for } \\
\text { VEGFR-2 on e-CTCs (\%) }\end{array}$ \\
\hline Breast & $42 / 50(84)$ & $42 / 50(84)$ \\
\hline Prostate & $30 / 30(100)$ & $30 / 30(100)$ \\
\hline Colorectal & $45 / 49(91.8)$ & $47 / 49(96)$ \\
\hline Lung & $27 / 34(79)$ & $27 / 34(79)$ \\
\hline
\end{tabular}

\section{ZTH-P07}

Responding to the necrosis-associated factor S100A4, MSCs induce an immunosuppressive environment by polarizing lymphocytes to Tregs

\section{${ }^{*}$ R. Lotfi',2, D. Zech', T. Yildiz', B. Jahrsdörfer ${ }^{2}, H$.} Schrezenmeier ${ }^{1,2}$

${ }^{1}$ Universitätsklinikum UIm, Institut für Transfusionsmedizin, UIm, Germany ${ }^{2}$ Institut für Klinische Transfusionsmedizin (IKT), Ulm, Germany

Background: Necrosis with the subsequent release of "damage associated molecular patterns" (DAMPs) is a characteristic feature of advanced tumor due to: 1) inadequate blood supply to the tumor 2) host cytotoxic immune response 3) elimination of programmed cell death (apoptosis) by the tumor itself. We have published data demonstrating that DAMPs induce immunosuppressive dendritic cells (DCs), on the one hand, and attract mesenchymal stromal cells (MSCs), on the other hand. We now sought to determine the impact of necrotic material (DAMPs) on biologic activity of MSCs focussing on their immunosuppressive capacities.

Methods: Perpheral blood mononuclear cells (PBMCs) were isolated from whole blood drawn from healthy donors. Anti-CD3/CD28 beads were used to induce the proliferation of lymphocytes within PBMCs. In the presence or absence of anti-CD3/CD28 beads and S100A4, PBMCs were co-cultured with human mesenchymal stromal cells (MSCs) for 5 days and intracellular expression of indoleamine dioxygenase (IDO) within CD4+ lymphocytes was assessed by FACS analysis.

Results: The ratio of CD4+IDO+ lymphocytes among PBMC increased over $100 \%$ when lymphocytes proliferated in the presence of MSCs and S100A4 $(125 \mathrm{ng} / \mathrm{ml})$ compared to the condition without S100A4. We made a similar observation when lymphocytes without any proliferative impulse were co-cultured with MSCs and S100A4, indicating that the presence of MSCs in combination with S100A4 induces Treg polarization in the resting as well as in the proliferating lymphocyte pool.

Conclusion: In the presence of MSCs, necrosis within tumor tissue impacts lymphocyte polarisation and thus probably the adaptive immune response to tumor. We characterized $\mathrm{S} 100 \mathrm{~A} 4$ as a specific necrosis-associated factor which is responsible for the immunoregulatory impact of DAMPs within tumor tissue. Underlying mechanisms of such a necrosis-induced immunosuppression need to be further characterized in order to develop strategies to impact the tumor microenvironment which is crucial for tumor growth and proliferation. 
ZTH-P08

\section{Tumor necrosis promotes further tumor proliferation while inhibiting the proliferative lymphocyte response in a manner which is independent of induction of regulatory T cells}

${ }^{\star}$ R. Lotfi',2, D. Zech' ${ }^{1}$, T. Yildiz' ${ }^{1}$ B. Jahrsdörfer ${ }^{2}$, C. Nienhaus ${ }^{1}$, H. Schrezenmeier ${ }^{1,2}$

${ }^{U}$ Universitätsklinikum Ulm, Institut für Transfusionsmedizin, Ulm, Germany ${ }^{2}$ Institut für Klinische Transfusionsmedizin (IKT), Ulm, Germany

Background: Necrosis with the subsequent release of "damage associated molecular patterns" (DAMPs) is a characteristic feature of advanced tumor. We have published data demonstrating that DAMPs induce immunosuppressive dendritic cells (DCs), on the one hand, and attract immunoregulatory mesenchymal stromal cells (MSCs), on the other hand. We now sought to determine the impact of necrotic material (DAMPs) on tumor growth and lymphoproliferative immune response

Methods: Soluble DAMPs were obtained from colorectal cell lines (HCT-116 and CACO-2) which were lysed by repeated freezing and thawing cycles. Peripheral blood mononuclear cells (PBMC) were obtained by density gradient separation of whole blood drawn from healthy donors. CD4+ cells were isolated from PBMC using MACS procedure. For proliferations assays, PBMC was stained with CFSE and proliferation was induced by adding anti-CD3/CD28 beads to the cell culture, after 5 days cell division was assessed by FACS. The expression of CD4, CD25, as well as intracellular indoleamine dioxygenase (IDO) and IL-10 was assessed by FACS analysis. Proliferation of tumor cells responding to necrotic material was assessed by measuring DNA content using CyQuant assay.

Results: Necrotic material (DAMPs) obtained from HCT-116 cells enhanced the proliferation of the same cell line up to 7 fold while its activity was significantly decreased following oxidation. On the other hand, the same necrotic material inhibited the proliferation of PBMC and CD4+ lymphocytes about $30 \%$ compared to the condition with anti-CD3/CD28 beads alone. Interestingly, the expression of markers which are associated with regulatory $\mathrm{T}$ cell (Treg) functions like CD25, intracellular IDO, and IL-10 were not influenced by DAMPs indicating that the mechanisms involved in the DAMPs-induced inhibition of lymphocyte proliferation is not due to activation of Tregs.

Conclusion: Necrosis within tumor tissue impacts lymphocyte proliferation and thus probably the adaptive immune response to tumor, while it further accelerate the proliferation of the tumor directly. Underlying mechanisms of necrosis-induced immunosuppression concurrent with enhanced tumor proliferation need to be further characterized in order to develop strategies to impact the tumor microenvironment which is crucial for tumor growth and proliferation. Based on our results, providing an oxidative tumor microenvironment may offer a therapeutic option.

\section{ZTH-P09}

\section{Screening and enumeration of virus- and tumor associated antigen-specific CD8 ${ }^{+} \mathrm{T}$ lymphocytes using streptamer technology and single-platform flow cytometry assay}

\section{*S. Matko ${ }^{1}$, M. Teichert ${ }^{1}$, M. Odendahl ${ }^{1}$, M. Wohsmann ${ }^{1}$,} M. Schmitz ${ }^{2}$, M. Bornhäuser ${ }^{3}$, T. Tonn ${ }^{1}$

'DRK-Blutspendedienst Nord-Ost, Experimentelle Transfusionsmedizin, Dresden, Germany

${ }^{2}$ Technische Universität Dresden, Institut für Immunologie, Dresden, Germany

3Universitätsklinikum Carl Gustav Carus, Medizinische Klinik

I-Stammzelltransplantation, Dresden, Germany

Background: Adoptive cell therapy has become an important component of virus-refraction-treatment after allogeneic stem cell transplantations (ASCT) supported by the advent of new technologies in cell separation, e.g. the reversible MHC class I-peptide streptamer technology. Enabling direct visualization and efficient enrichment of antigen-specific cytotoxic $\mathrm{CD}^{+}$T-Lymphocytes (CTLs) MHC-class I-peptide streptamer technology may allow the identification of rare tumor associated antigen WT-1 specific CTLs which can be used for the treatment of AML-relapse-patients after ASCT.

Methods: Rare-cell-detection of virus- and WT1-antigen associated CTLs was realized by the use of MHC class I-peptide streptamer technology (IBA Stage, Göttingen, Germany). To examine quality and sensitivity of this method, PBMCs containing a certain number of CMV pp65 A*0201 (NLVPMVATV) specific CTLs were titrated in blood without CMV-specific CTLs, using single-platform flow cytometry and True Count tubes (BD Biosciences, San Jose, CA, USA) with the benefit to assess absolute cell numbers of streptamer positive cells within blood samples and products. To validate reproducibility, intra-assay-deviation was calculated after application of the MHCI-multimer CMV pp65 B*0702 (TPRVTGGGAM) in ten separate stainings using the same donor-material. The applied Streptamer for donor-screening of WT1-positive CTLs is HLA A*0201 restricted, presenting the peptide RMFPNAPYL.

Results: Quality control of streptamer staining through titration of antigen-specific CTLs showed a linearity of $r^{2}>0.99$ and a detection limit as low as 1 CMV pp65-specific CTL per microliter (correlating with a frequency of $0.032 \pm 0.0033$ ). Also the intra-assay-deviation stated an applicable frequency of $0.6 \pm 0.03$ with a random error of $5 \%$. In contrast screening results for 88 HLA A*0201 positive healthy donors with a mean frequency of 0.003 and a standard deviation of 0.005 accounting for WT-1 specific CTLs corresponded to background noise. Even the maximum frequency of $0.026 \pm 0.005$ did not state a distinct population. In comparison CMV A*0201 pp65/B*0702 pp65 screening in the same group of donors showed a mean value of $0.219 \pm 0.899 / 0.784 \pm 1.602$ and a maximum frequency of $7.596 \pm 0.899 / 5.175 \pm 1.602$, respectively.

Conclusion: The MHC class I-peptide streptamer technology for the detection of rare antigen-specific CTLS is a reliable technique with high sensitivity and reproducibility enabling the direct enumeration of antigen specific CTLs within peripheral blood and blood products. Taking into account, we were only able to show a linear detectability of virus-specific CTLs in a representative number of standard donors, our data indicate that the determined frequency of WT-1 specific CTLs found among healthy donors is well below the detection limit of MHC class I-petide streptamer staining.

\section{ZTH-P10 \\ Generation of a platelets and granulocytes rich Plasma for the treatment of Sepsis}

${ }^{*}$ M. Fahrendorff, D. Preys, M. Biehl, W. Böcker

Fresenius Kabi Deutschland GmbH, R\&D, Friedberg, Germany

Background: Sepsis is a severe medical condition that can be characterized as a whole body inflammatory state. It can be thought of as falling within a continuum from infection to multiple organ dysfunction syndromes. Over the last years, systemic antibiotics have not provided an effective treatment against infections. Today there is growing evidence that local activity of immuno competent cells is able to reduce or even prevent both bacteria colonization of an implant and biofilm formation.

Methods: Based on this background it is advisable and desirable to have a fast, reproducible and easy way for the production of platelet and granulocyte rich plasma (PGRP). That is the reason for the optimization of the Plasmasequestration Procedure (PSQ) in the Continuous Autotransfusion System (C.A.T.SPlus). C.A.T.SPlus device works on the principle of a continuous flow centrifuge comparable to continuous systems for hemapheresis which have been widely used in blood banks for decades. The optimized PSQ procedure is for separation of whole blood into packed red cells (PRC), plasma (PLS) as well as PGRP.

Results: The recovery for the granulocytes in the PGRP fraction reaches approx. $50 \%$ and for the platelets approx. $60 \%$.

A large body of data now exists, that show how leukocytes and particularly polymorphonuclear neutrophils (PMNs) play a significant role in 
fighting against implant-related infections, through a variety of mechanisms [Bettin et al.].

Conclusion: Some authors conclude from their studies that lower local levels of PMNs in the early surgical wound are directly related to the subsequent occurrence of septic complications and higher early local leukocyte concentrations play a significant protective role against post-surgical infection [Bettin et al.].

The preparation of PGRP with the optimized PSQ procedure is safe, easy, fast and economic. Providing reproducible quality PGRP with this optimized procedure will allow to design broader clinical studies to show the benefit of the proposed therapy.

\section{ZTH-P11}

\section{Defined mesenchymal stem cells inhibit experimental gramnegative pneumonia and acute lung injury}

*I. Schevtschenko ${ }^{1}$, N. Baal', A. Wachtendorf', A. Lippitsch", S. Kranz', G. Bein' ${ }^{1}$, C. Brendel' ${ }^{2}$, H. Hackstein ${ }^{1}$

${ }^{1}$ University of Gießen, Clinical Immunology and Transfusion Medicine, Gießen, Germany

${ }^{2}$ University of Marburg, Marburg, Germany

Background: Most information regarding immunomodulatory mesenchymal stem cell (MSC) function are generated from studies expanding heterogenous stem cell populations of adherent cells. Major disadvantages of these methods are leukocyte contamination and cell culture heterogeneity. Here, we have expanded prospectively defined PDGFR $\alpha^{+}$SCA$1^{+} \mathrm{CD} 45^{-}$TER $119^{-}$MSC from murine bone marrow by high purity FACS sorting. Therapeutic activity of defined MSC was analysed in vivo after induction of acute Klebsiella pneumonia infection. Klebsiella pneumonia represents a leading cause of hospital-acquired respiratory tract infections and death.

Methods: PDGFR $\alpha^{+} \mathrm{SCA}-1^{+} \mathrm{CD} 45^{-}$TER119- $\mathrm{MSC}$ representing $<0.1 \%$ of bone marrow cells were sorted from C57BL6 mice to high purity $(>98 \%)$ and expanded in vitro with human platelet lysate. $4 \mathrm{~h}$ after induction of acute murine respiratory Klebsiella pneumonia infection animals were treated intra-tracheally with defined MSC $\left(1 \times 10^{6}\right)$ or received a mock $\mathrm{NaCl}$ treatment. Clinical pneumonia parameter were assessed on d2 post infection.

Results: MSC treatment significantly inhibited acute lung injury as indicated by significantly decreased cellular alveolitis and decreased alveolar protein leakage. Moreover, bronchoalveolar pro-inflammatory TNF- $\alpha$ and IL-12p70 production were significantly decreased whereas immunoregulatory IL-10 production was not affected. Dissection of alveolar and lung parenchyma leukocyte subsets by flow cytometry revealed a significant reduction of neutrophils and dendritic cells after MSC treatment. Additionally, analysis of costimulatory surface molecule expression on lung DC revealed a significant suppression of CD86 expression on CD103 DC, plasmacytoid DC and CD11 bi DC after MSC treatment.

Conclusion: To our knowledge this is the first report demonstrating anti-inflammatory activity of phenotypically defined, prospectively isolated MSC in a clinically relevant in vivo model. Prospective isolation of defined MSC populations may facilitate our understanding of MSC biology and the development of GMP-compliant MSC protocols for cell therapy.

\section{ZTH-P12 \\ Microbiological Colonization of Donor Corneas after Disinfection with PVP lodine}

\section{*J. Schroeter, F. Herrlinger, I. Wilkemeyer, A. Pruß}

Charité - Universitätsmedizin Berlin, Inst. für Transfusionsmedizin, Universitätsgewebebank, Berlin, Germany

Background: Donor corneas are physiologically colonized with microorganisms and cannot be sterilized without destruction of their biological functions. Therefore disinfection and antibiotic treatment of the tissue are necessary.

Methods: Conjunctiva and intraocular swabs were taken from 60 cornea donor eyes after periocular skin disinfection with 7,5\% PVP iodine and ocular surface disinfection with $2 \% \mathrm{PVP}$ iodine solution. After rinsing of the cornea and conjunctiva with $0,9 \% \mathrm{NaCl}$ solution an in situ corneoscleral disc excision was performed and the donor corneas were put into organculture. The swabs were incubated in Thioglycolat solution for a maximum of 14 days at $32{ }^{\circ} \mathrm{C}$

Results: A microbiological colonization was found in 51 of the 60 donor eyes $(85 \%) .106$ out of 240 swabs showed a microbial growth. In contrast only 2 donor corneas $(1.7 \%)$ showed microbiological contamination of their culture medium. In 27 of the 51 donor corneas which showed microbial colonization the microorganisms found were of higher potential virulence. None of the corneal grafts led to an infection of the recipient eye. Conclusion: The rate of microbial colonization of donor corneas is high, while the rate of contaminated culture medium is low. It remains questionable that a microbiological test of the ocular surface before donor cornea explanation can help reducing the risk of infections, especially endophalmitis in the recipient. 